\title{
Probabilistic Analysis Software for Structural Seismic Response
}

\author{
by \\ Brian David Sjoberg \\ B.Sc., The University of Calgary, 1988 \\ M.Sc., The University of Calgary, 1999 \\ A THESIS SUBMITTED IN PARTIAL FULFILLMENT OF THE \\ REQUIREMENTS FOR THE DEGREE OF \\ DOCTOR OF PHILOSOPHY \\ in \\ THE FACULTY OF GRADUATE STUDIES \\ (Department of Civil Engineering)
}

We accept this thesis as conforming to the required standard

THE UNIVERSITY OF BRITISH COLUMBIA

October 2003

(C) Brian David Sjoberg, 2003 
In presenting this thesis in partial fulfilment of the requirements for an advanced degree at the University of British Columbia, I agree that the Library shall make it freely available for reference and study. I further agree that permission for extensive copying of this thesis for scholarly purposes may be granted by the head of my department or by his or her representatives. It is understood that copying or publication of this thesis for financial gain shall not be allowed without my written permission.

Department of Livil Enginpering.

The University of British Columbia

Vancouver, Canada

Date oct.10,2003 


\section{ABSTRACT}

The evaluation of seismic reliability of building structures is a complex and computationally expensive process since it requires, at the most fundamental level, the evaluation of the probabilistic dynamic response of a given structure to the stochastic dynamic action of an earthquake. Because of the difficulty of determining the response of a structure in a statistical sense, past estimates of the seismic reliability of existing structures, and typical structural systems, have been largely qualitative in nature. With the movement of many national building codes towards more performance-based design measures, a need was identified for a more quantitative method of evaluating structural reliability under seismic loading.

To meet this need, a new software application called PSResponse was developed that gives engineers and researchers the ability to rigorously evaluate the probable effect of a wide range of ground motion characteristics and structural model parameters, each with their own random nature, on the dynamic response of a structure. The mathematical modeling methods forming the foundation of the software architecture were selected following a comprehensive review of random vibration methods and numerical procedures that assessed their suitability for analyzing the probabilistic seismic response of civil engineering structures. That review determined that the frequency-domain based random vibration methods are too restrictive in their inherent assumptions to confidently apply their results to real structures experiencing realistic earthquakes. Instead, a numerical time-history approach incorporating the Monte Carlo method provides a robust, accurate and straightforward means of evaluating the probabilistic response of a structure without regard to the degree of non-linearity in the restoring force, complexity of the structural system, nature of the variability in structural properties or nature of the random excitation process.

As part of the software development process, a new algorithm for parameter identification of the well-known BWBN, or Bouc-Wen, hysteresis model was developed, which included a modification to the function controlling pinching behaviour 
to simplify the parameter identification process. The number of pinching parameters was reduced from six to three, which has the added benefit that the role of each of the three new parameters is more easily understood than the relationship between the six parameters of the original pinching function.

Following development of the beta version of PSResponse, two case studies were completed that demonstrated the capabilities of the software as a research and analysis tool. These case studies provided for the first time a probabilistic analysis of the importance of the hysteresis assumption in inelastic analysis, the accuracy of the wellknown equal displacement observation in structural dynamics and the relative effect of random structural properties on elastic dynamic response. Results showed that the hysteretic behaviour of a structure needs to be accurately modeled, particularly in shorter natural period structures, to provide an accurate probabilistic description of response and hence a good estimate of seismic structural reliability. Also, the equal displacement principle is valid in the sense that elastic peak displacement provides a generally conservative first approximation of inelastic peak displacement, which in turn results in a generally conservative prediction of reliability. Finally, case study results showed that the characteristics and randomness of ground motion records has a much larger influence than structural randomness on the probabilistic dynamic response of a structure. Therefore, once a suitable seed record has been selected, the peak response probability distributions for a given structural model could be applied to a real structure with reasonable confidence since the assumed level of uncertainty in the structural parameters needs to be only approximately correct. However, for strength related limit state evaluations related to peak response, structural variability still has an important effect. 


\section{ACKNOWLEDGEMENTS}

I gratefully acknowledge the support of my supervisor, Dr. Helmut Prion, throughout the course of this project. His encouragement, financial support and generosity in providing resources to aid in software development were much appreciated.

I would also like to thank the members of my advisory committee; Dr. Ricardo Foschi, Dr. Frank Lam, Dr. Reza Vaziri and Dr. Carlos Ventura who acted as both my committee members and teachers throughout my years at UBC. Their teaching efforts and generosity with their time were always appreciated. In particular, the comments and suggestions of Dr. Foschi and Dr. Ventura during the final development phase of PSResponse were encouraging and insightful.

Finally, I would like to thank my fiancée, Karen Evans, both for her support and understanding throughout my graduate studies, as well as her invaluable assistance in the development of PSResponse. 
To Barry \& Elaine Sjoberg 


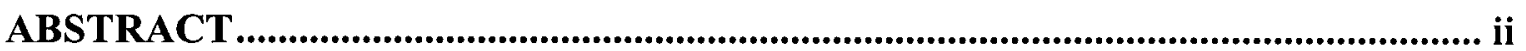

ACKNOWLEDGEMENTS .........................................................................................

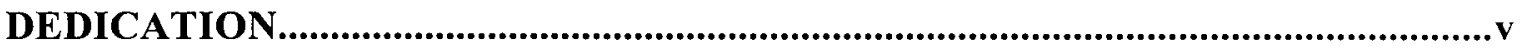

TABLE OF CONTENTS ........................................................................................

LIST OF TABLES

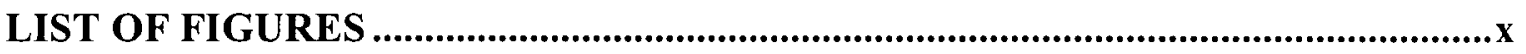

CHAPTER 1: INTRODUCTION......................................................................................

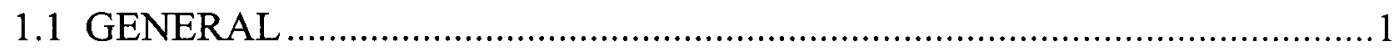

1.2 OBJECTIVE

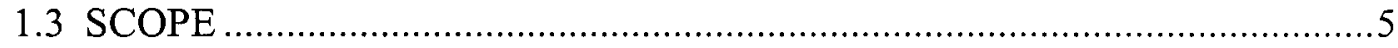

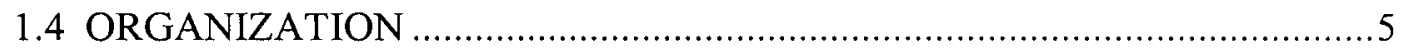

CHAPTER 2: LITERATURE REVIEW .......................................................................

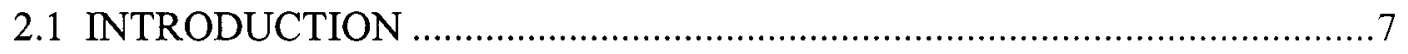

2.2 MATHEMATICAL MODELING OF STRUCTURAL BEHAVIOUR ….......8

2.2.1 Analytical Methods .......................................................................

2.2.1.1 Markov Methods Based on the Fokker-Planck-

Kolmogorov Equation......................................................... 9

2.2.1.1.1 General Random Vibration Theory and Markov Process Theory...........................................9

2.2.1.1.2 Solution Methods for the Fokker-Planck

Kolmogorov Equation .........................................17

2.2.1.1.2.1 Galerkin Based Methods ....................18

2.2.1.1.2.2 Finite Element Method .......................18

2.2.1.1.2.3 Closure Techniques ...........................19

2.2.1.1.2.4 Stochastic Averaging .........................21

2.2.1.1.2.5 Numerical Diffusion.........................24

2.2.1.1.3 First-Passage Problem Solution Methods.............27

2.2.1.1.3.1 Assumption of Independent

Events ................................................28

2.2.1.1.3.2 Markov Process Theory …………........33

2.2.1.2 Perturbation Method ...............................................................

2.2.1.3 Equivalent Linearization Method.........................................36

2.2.1.4 Functional Series Representation..........................................40 


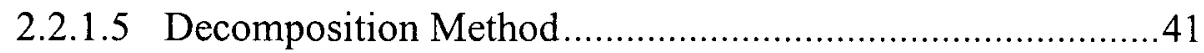

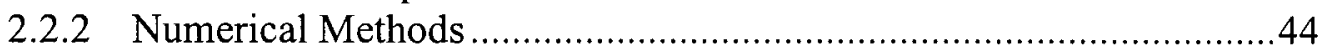

2.2.2.1 Direct Monte Carlo Simulation.............................................45

2.2.2.2 Selective Monte Carlo Simulation ........................................46

2.2.2.2.1 Double and Clump.............................................47

2.2.2.2.2 Russian Roulette and Splitting ……..................... 48 .

2.2.2.2.3 Latin Hypercube Sampling ..................................49

2.2.2.3 Response Surface Method...................................................50

2.3 EARTHQUAKE GROUND MOTION MODELS ......................................53

2.3.1 Filtered White-Noise and Filtered Poisson Process Models ................54

2.3.2 Spectral Representation Method .....................................................57

2.3.3 Stochastic Wave Theory ……….....................................................6

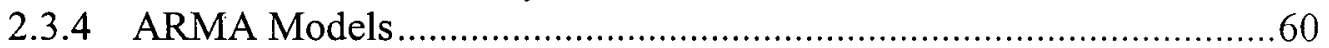

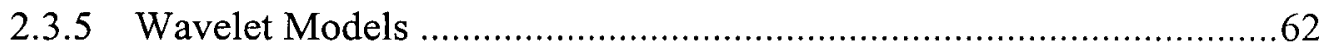

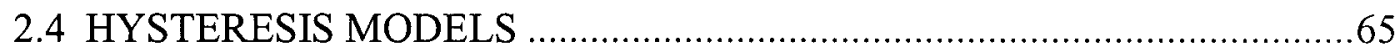

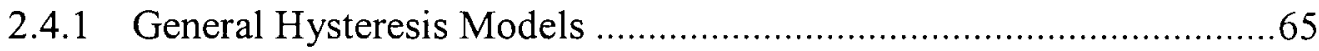

2.5 SEISMIC STRUCTURAL RESPONSE AND RELIABILITY STUDIES ....70

CHAPTER 3: NUMERICAL ALGORITHM DEVELOPMENT …..........................75

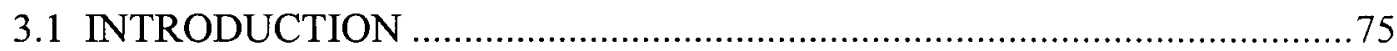

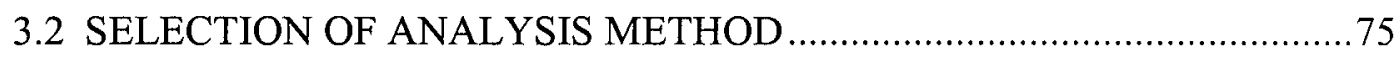

3.3 NUMERICAL MODELS AND SOLUTION METHODS …........................77

3.3.1 Numerical Time-Stepping Method ……………..............................77

3.3.2 Time-Stepping Overshoot Problem..................................................

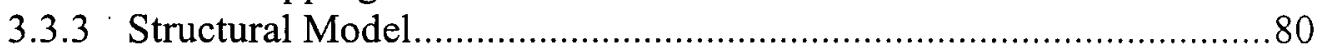

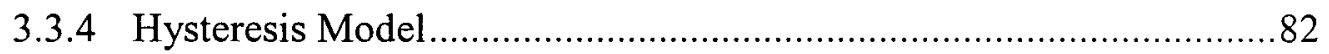

3.3.4.1 Modification to the BWBN Model ………........................82

3.3.4.2 Numerical Solution of Hysteresis Model................................85

3.3.4.3 Consideration of Degrading Natural Frequency ...................88

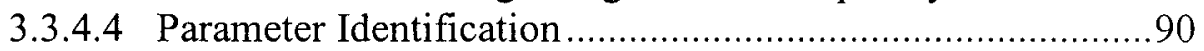

3.3.4.5 Parameter Adjustment for MDOF Structures ......................96

3.3.5 Fourier Analysis and Power Spectrum Estimation ............................96

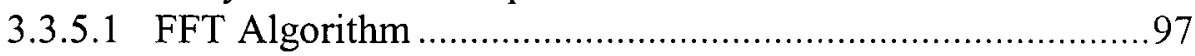

3.3.5.2 Power Spectrum Algorithm ............................................98

3.3.6 Acceleration Record Filtering ......................................................... 104

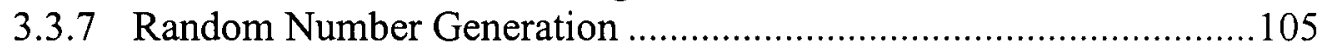

CHAPTER 4: SOFTWARE FRAMEWORK AND USER-INTERFACE ...............109

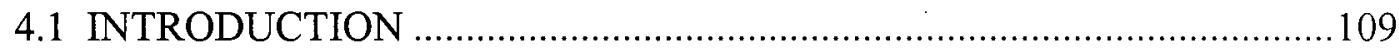

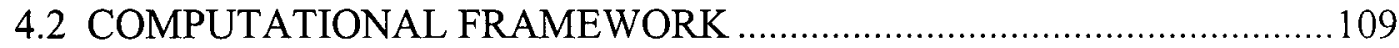

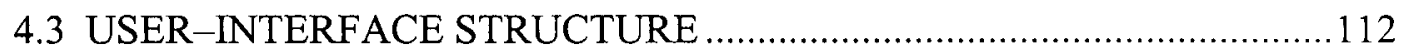

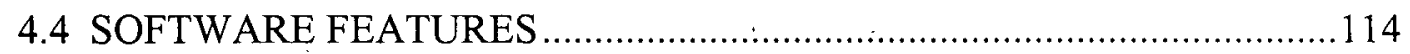

4.4.1 General Input Parameters Dialog Box ..............................................116

4.4.2 Multiple Earthquake Analysis Parameters Dialog Box ....................117 
4.4.3 Single-Degree-of-Freedom Properties Dialog Box...................... 118

4.4.4 Multiple-Degree-of-Freedom Shear Properties Dialog Box ........... 119

4.4.5 Multiple-Degree-of-Freedom Frame Properties Dialog Box ..........120

4.4.6 Probability Distribution Example Dialog Box ..............................121

4.4.7 Hysteresis Parameter Identification Dialog Boxes ......................... 122

4.4.8 Modal Damping Parameters Dialog Box .................................... 125

4.4.9 MDOF Damping Parameters Dialog Box ......................................127

4.4.10 Oscillation Motion Parameters Dialog Box ..................................... 128

4.4.11 Single Ground Motion Parameters Dialog Box .............................. 129

4.4.12 Ground Motion Generation Parameters Dialog Box (Filtered)........ 131

4.4.13 Ground Motion Generation Parameters Dialog Box (Real)............. 133

4.4.14 Elastic Responses Dialog Box .................................................... 135

4.4.15 SDOF Inelastic Responses Dialog Box ........................................ 136

4.4.16 MDOF Inelastic Responses Dialog Box ...................................... 137

4.4.17 Plot Modal Results Dialog Box................................................... 138

4.4.18 Plot SDOF Results Dialog Box................................................... 141

4.4.19 Plot MDOF Results Dialog Box ................................................ 143

4.4.20 Print Results Dialog Box.......................................................... 146

CHAPTER 5: SOFTWARE VERIFICATION AND CASE STUDIES .................151

5.1 INTRODUCTION ..............................................................................151

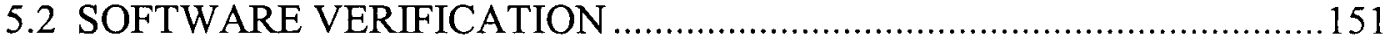

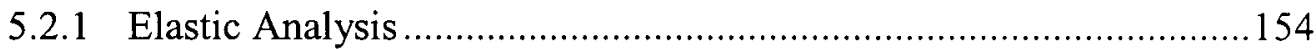

5.2.1.1 Single-Degree-of-Freedom Systems .........................154

5.2.1.2 Multiple-Degree-of-Freedom System ............................ 156

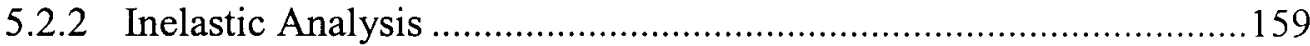

5.2.2.1 Single-Degree-of-Freedom Systems ............................1159

5.2.2.2 Multiple-Degree-of-Freedom System ........................... 164

5.3 CASE STUDIES ........................................................................... 170

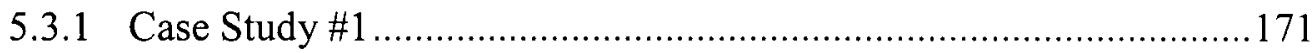

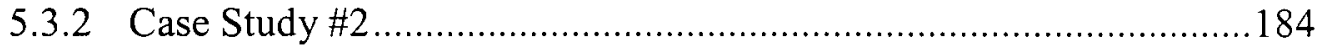

CHAPTER 6: SUMMARY, CONCLUSIONS AND RECOMMENDATIONS .....210

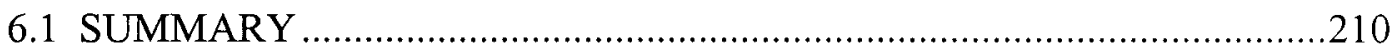

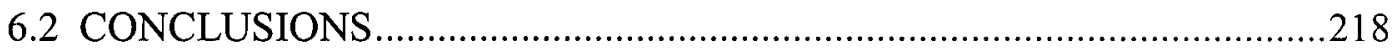

6.3 RECOMMENDATIONS FOR FURTHER RESEARCH ........................222

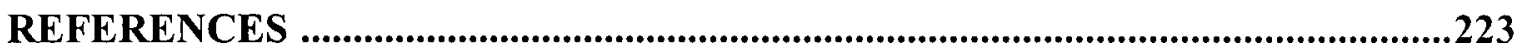




\section{LIST OF TABLES}

Page

Table 3.1 Power Spectrum Frequency Resolution .............................................. 101

Table 5.1 Comparison of Peak Deformation Responses of SDOF Systems ............156

Table 5.2 Comparison of Elastic Peak Responses of Five-Storey Shear Frame........158

Table 5.3 Comparison of Inelastic Peak Responses of SDOF Systems .................. 163

Table 5.4 Comparison of Inelastic Peak Responses of Five-Storey Shear Frame ..... 165

Table 5.5 Peak Response Statistics of a Five-Storey Elastic Shear Frame...............172

Table 5.6 Elastic Peak Response Distribution Comparison Statistics......................177

Table 5.7 Statistics for the Percentage Difference between RHA \& RSA Results .... 179

Table 5.8 Hysteresis Model Descriptions.......................................................... 186

Table 5.9 SDOF Peak Deformation Response Statistics ..................................... 187

Table 5.10 Gumbel Distribution Parameters for Peak Deformation ...........................199

Table 5.11 Displacement (Drift) Limit Exceedence Probabilities..............................200

Table 5.12 Displacement (Drift) Limit Reliability Indices ...................................201

Table 5.13 Percentage Difference Statistics Inelastic vs. Elastic Peak Deformation ...204

Table 5.14 Gumbel Distribution Parameters for Percentage Difference Results ........205

Table 5.15 Zero Percent Difference Exceedence Probabilities ...............................206 


\section{LIST OF FIGURES}

Page

Fig. 2.1 System Response and White Noise Approximation .................................. 14

Fig. 2.2 First-Passage Time and Domain Barriers in the Phase Plane .....................28

Fig. 2.3 Filtered White-Noise Power Spectrum ..................................................55

Fig. 2.4 Example Amplitude Modulating Function ............................................ 56

Fig. 3.1 BWBN Model Pinching Function Comparison ........................................86

Fig. 3.2 Exact Solution of Non-Pinching, Non-Degrading BWBN Model................87

Fig. 3.3 Square Window, Bartlett Window and Fourier Transforms ...................... 102

Fig. 4.1 Computational Framework - Initialization .......................................... 110

Fig. 4.2 Computational Framework - Multiple Record Analysis (Filtered) .............111

Fig. 4.3 Computational Framework - Multiple Record Analysis (Real) .................111

Fig. 4.4 Computational Framework - Run Multiple Records .................................112

Fig. 4.5 Computational Framework - Solvers ................................................113

Fig. 4.6 Computational Framework - Generate Random Properties .....................114

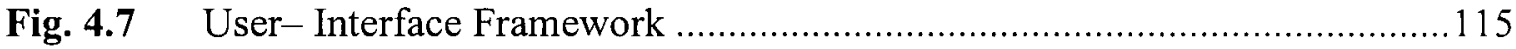

Fig. 4.8 General Input Parameters Dialog Box ..................................................116

Fig. 4.9 Multiple Earthquake Analysis Parameters Dialog Box ............................. 117

Fig. 4.10 Single-Degree-of-Freedom Properties Dialog Box.................................118

Fig. 4.11 Multiple-Degree-of-Freedom Shear Properties Dialog Box .....................119

Fig. 4.12 Multiple-Degree-of-Freedom Frame Properties Dialog Box..................120

Fig. 4.13 Probability Distribution Example Dialog Box ......................................121

Fig. 4.14 Hysteresis Parameter Identification Dialog Box (Quasi-Static Data) ......... 122

Fig. 4.15 Hysteresis Parameter Identification Dialog Box (Acceleration Data) ......... 124

Fig. 4.16 Modal Damping Parameters Dialog Box ............................................... 125

Fig. 4.17 Modal Properties Pop-Up Dialog Box ................................................ 126

Fig. 4.18 MDOF Damping Parameters Dialog Box .............................................. 127

Fig. 4.19 Oscillation Motion Parameters Dialog Box .............................................. 128

Fig. 4.20 Single Ground Motion Parameters Dialog Box ..................................... 129

Fig. 4.21 Ground Motion Statistics Pop-Up Dialog Box........................................130

Fig. 4.22 Ground Motion Generation Parameters Dialog Box (Filtered) ..................131

Fig. 4.23 Ground Motion Generation Parameters Dialog Boxes (Real) ....................133

Fig. 4.24 Elastic Responses Dialog Box ................................................. 135

Fig. 4.25 Storey Selection Example Dialog Box....................................................136

Fig. 4.26 SDOF Inelastic Responses Dialog Box ................................................136

Fig. 4.27 MDOF Inelastic Responses Dialog Box ............................................ 137

Fig. 4.28 Plot Modal Results Dialog Box ...............................................................138

Fig. 4.29 Input Motion Time-History Pop-Up Dialog Box .................................... 139

Fig. 4.30 Input Spectrum Pop-Up Dialog Box ................................................... 140

Fig. 4.31 Response Spectra Pop-Up Dialog Box................................................. 140

Fig. 4.32 Modal Response Time-History Example Pop-Up Dialog Box................... 141 
Fig. 4.33

Fig. 4.34

Fig. 4.35

Fig. 4.36

Fig. 4.37

Fig. 4.38

Fig. 4.39

Fig. 4.40

Fig. 4.41

Fig. 4.42

Fig. 4.43

Fig. 4.44

Fig. 4.45

Fig. 5.1

Fig. 5.2

Fig. 5.3

Fig. 5.4

Fig. 5.5

Fig. 5.6

Fig. 5.7

Fig. 5.8

Fig. 5.9

Fig. 5.10

Fig. 5.11

Fig. 5.12

Fig. 5.13

Fig. 5.14

Fig. 5.15

Fig. 5.16

Fig. 5.17

Fig. 5.18

Fig. 5.19

Fig. 5.20

Fig. 5.21

Fig. 5.22

Fig. 5.23

Fig. 5.24

Fig. 5.25

Fig. 5.26

Fig. 5.27

Fig. 5.28

Fig. 5.29

Fig. 5.30
Plot SDOF Results Dialog Box..... 141

Hysteresis Parameter Identification Dialog Box (Quasi-Static Data) ......... 142

Hysteresis Parameter Identification Dialog Box (Acceleration Data) .......... 143

Plot MDOF Results Dialog Box

143

MDOF Response Time-History Example Pop-Up Dialog Box .................. 144

MDOF Response Time-History Example Pop-Up Dialog Box ................. 145

MDOF Response Time-History Example Pop-Up Dialog Box ................. 145

Print Results Dialog Box................................................................... 146

Analysis Summary Report ................................................................. 148

Random Natural Frequency Array Printed Output ................................. 149

Example Response Time-History Printed Output (Elastic Analysis) .......... 149

Response Spectra Data Printed Output ............................................... 150

Example Multiple Record Analysis Printed Output (Elastic Analysis)........ 150

SDOF Elastic Deformation Response - PSResponse vs. SAP2000 ............ 155

Five-Storey Elastic Shear Frame ........................................................ 156

Five-Storey Shear Frame Elastic Response - PSResponse vs. SAP2000 ...1157

SDOF Inelastic Deformation Response - PSResponse vs. SAP2000 .......... 160

SDOF Inelastic Shear Response - PSResponse vs. SAP2000 ......................161

SDOF Hysteresis Loops - PSResponse vs. SAP2000 ............................. 162

SDOF Dissipated Energy - PSResponse ................................................ 164

Five-Storey Inelastic Shear Frame ....................................................... 165

Five-Storey Shear Frame Inelastic Deformation Response -

PSResponse vs. SAP2000 ............................................................... 166

Five-Storey Shear Frame Inelastic Shear Response -

PSResponse vs. SAP2000.

Five-Storey Shear Frame Hysteresis Loops - PSResponse vs. SAP2000 ...168

Five-Storey Shear Frame Dissipated Energy - PSResponse.......................169

Base Shear Response Histograms .......................................................... 173

Base Moment Response Histograms................................................... 174

Storey 5 Displacement Response Histograms ......................................... 175

Peak Response Distributions.................................................... 176

Error Distribution for CQC \& SRSS vs. RHA Peak Responses .................. 183

El Centro Response Spectra .......................................................... 184

Cyclic Test of Parallam ${ }^{\circledR}$ Column and Best-Fit Hysteresis Model .............. 185

SDOF Hysteretic Response with $\mathrm{T}=8.0 \mathrm{sec}$, Damping $=2 \% \ldots \ldots \ldots \ldots \ldots \ldots . .187$

Displacement Response Histograms, $\mathrm{T}=0.1 \mathrm{sec}$, Damping $=2 \% \ldots \ldots \ldots \ldots . .189$

Displacement Response Histograms, $T=0.2 \mathrm{sec}$, Damping $=2 \% \ldots \ldots \ldots \ldots . .190$

Displacement Response Histograms, $\mathrm{T}=0.4 \mathrm{sec}$, Damping $=2 \% \ldots \ldots \ldots \ldots \ldots .191$

Displacement Response Histograms, $T=0.5 \mathrm{sec}$, Damping $=2 \% \ldots \ldots \ldots \ldots . .192$

Displacement Response Histograms, $\mathrm{T}=0.6 \mathrm{sec}$, Damping $=2 \% \ldots \ldots \ldots \ldots . .193$

Displacement Response Histograms, $\mathrm{T}=1.0 \mathrm{sec}$, Damping $=2 \% \ldots \ldots \ldots \ldots . .194$

Displacement Response Histograms, $T=3.0 \mathrm{sec}$, Damping $=2 \% \ldots \ldots \ldots \ldots . .195$

Displacement Response Histograms, $\mathrm{T}=8.0 \mathrm{sec}$, Damping $=2 \% \ldots \ldots \ldots \ldots \ldots . .196$

Hysteresis Model Comparison of Peak Deformation Distributions............. 198

Percentage Difference Histograms Inelastic vs. Elastic. 
Fig. 5.31 Percentage Difference Distributions Inelastic vs. Elastic .........................208 


\section{CHAPTER 1}

\section{INTRODUCTION}

\subsection{GENERAL}

The field of earthquake engineering has existed since the Middle Ages when many European, Chinese and Japanese structures were constructed with post and beam systems employing complex joinery and/or diagonal bracing for lateral load resistance. These construction methods, as well as lightweight timber materials, structural redundancy and even passive control devices, allowed structures to sustain large deformations under earthquake loading and effectively dissipate the input seismic energy. Using these techniques, some churches and temples that were constructed as much as 1000 years ago still stand today (Popovski 2000).

The successful seismic performance of these ancient structures is more a testament to the craftsmanship of the original builders and generations of experience than to a fundamental understanding of seismic loading on soils and structures. The field of earthquake engineering, as we know it today, really began developing in the latter half of the $20^{\text {th }}$ century as seen from the first inclusion of seismic loading provisions in the main text of the National Building Code of Canada (NBCC) in 1953. These provisions, which specify both the seismic design forces and the design and detailing requirements for lateral load resisting structural systems, have been updated approximately every five years since 1965 resulting in the current 1995 version of the NBCC.

In the current version of the NBCC the seismic design provisions provide estimates of peak ground acceleration and peak ground velocity for various regions of the country resulting from an earthquake that has a 10 percent probability of exceedence in 50 years. These ground motion parameters are then used in conjunction with simple 
formulae to determine a distribution of static lateral forces for which the structure should be designed. The resulting forces are considered equivalent to the dynamic forces of an earthquake and any structure designed to resist these forces should be able to resist minor . levels of earthquake ground motion without damage, resist moderate levels of earthquake ground motion without structural damage but possibly with some non-structural damage, and resist major levels of earthquake ground motion without collapse but with some structural as well as non-structural damage. These qualitative levels of performance are contingent on the design allowing for stress reversals, providing adequate member ductility and providing connections with adequate strength and resilience. Included in the various formulae used to determine the design lateral forces are the use of several factors to account for; inelastic behaviour (R), relative importance of the structure (I), and site soil effects $(\mathrm{F})$.

The prescriptive procedures and various factors outlined in the 1995 NBCC are based on years of past proven experience and are easy to apply. As it concerns a matter of life safety, this approach has been justified on the grounds that any major change in building practice can lead to unexpected risks. It was this view that led to the introduction of a calibration factor, the so-called 'over-strength' factor $(U=0.6)$, in the $1990 \mathrm{NBCC}$ to maintain the same seismic design forces when a new formulation for base shear was adopted. The drawback of a prescriptive procedure is that it oversimplifies a very complex problem and it does not allow the reliability of a design to be quantified.

In 1992, the Canadian National Committee on Earthquake Engineering (CANCEE), which has the responsibility of preparing and recommending the seismic loading provisions of the NBCC, recognized that major changes would be necessary for the 2000 NBCC. A resolution was approved which stated (Heidebrecht et al. 1995):

"that CANCEE place a very high priority on a major redevelopment of the seismic loading provisions of the 2000 edition of $\mathrm{NBCC}$, with particular emphasis on: 
1. Developing a format suitable for utilizing seismic hazard expressed as uniform hazard spectra,

2. Evaluating the appropriateness of the level of protection (expressed as the minimum lateral seismic force) by comparison with that used in various U.S. codes (UBC, SEAOC and NEHRP), including comparisons of seismic hazard determined at points along the Canada-U.S. border.

and that CANCEE maintain close linkages with various U.S. code development projects in order to benefit from their experience".

To implement this resolution, CANCEE established the NBCC 2000 Task Force to generate a redevelopment plan and coordinate code development work. In 1993, the task force published a list of six major issues that needed to be addressed:

1. Seismic loading format suitable for utilizing spectral ordinates determined from seismic hazard analysis,

2. Evaluation of the current level of protection,

3. Role of different design (or performance) levels in the code,

4. Development of direct site spectra to recognize different site soil conditions,

5. Development of design requirements for low to moderate seismic hazard zones,

6. Explicit or implicit recognition of over-strength in seismic design.

In recognizing these issues, CANCEE is clearly moving the NBCC towards a performance-based design code, perhaps with the intent of establishing specific probabilistic performance targets for a code designed structure in a seismic event. To enable the evaluation of structural performance, the current design objectives of: no damage, moderate structural damage and prevention of collapse in the event of minor, moderate and major levels of earthquake ground motion, respectively, need to be expressed as limits on quantitative terms such as: a damage index, overall lateral deflection or inter-storey drift. In addition, the evaluation of performance, and 
specifically the level of protection, implies the use of reliability-based methods to express the risk of non-performance (i.e. exceedence of limits) in probabilistic terms.

\subsection{OBJECTIVES}

Evaluating the reliability or risk of non-performance of a structural system under seismic loading requires, at the most fundamental level, a method to evaluate the probabilistic dynamic response of a given structure to the stochastic dynamic action of an earthquake. Once the response of the structure is known in a statistical sense, the task of determining structural reliability is a matter of using the statistical description of structural response to determine the probability of exceeding the chosen limits on the quantitative terms being used to assess structural performance.

Evaluation of the probabilistic response of a structure to stochastic dynamic loading is a complex and computationally expensive process. For this reason past estimates of the seismic reliability of existing structures, and typical structural systems, have been largely qualitative in nature. With the movement towards more performancebased design codes a need exists for a more rigorous and convenient method of evaluating structural reliability under seismic loading. This need is the basis for the present research project, which has the following objectives:

1. Evaluate the various analytical and numerical methods that have been developed to predict the response of linear and non-linear systems under stochastic dynamic actions.

2. Identify a method suitable for probabilistic analysis of the seismic response of civil engineering structures.

3. Develop software to enable application of the chosen probabilistic analysis method for use by engineers and researchers in evaluating structural reliability under seismic loading. 


\subsection{SCOPE}

Since the objectives of this study relate to the evaluation of structural reliability under seismic loading, it is given that the study and related software development focus on probabilistic dynamic response of structures to earthquake-induced ground acceleration events. Dynamic loading in the form of wind-induced vibration, blast loading or impact loading is not considered, although the mathematical descriptions of the structural response to those types of loading are very similar. In addition, the type of structures that are considered in this study are those that may be represented by typical lumped mass models consisting of shear walls or frames as the lateral load resisting elements. Irregular structures consisting of complex geometry with significant distributed mass are outside the scope of this investigation.

\subsection{ORGANIZATION}

This thesis contains six chapters that are divided as follows; Chapter 2 contains a literature review of the research related to different aspects of modeling the response of linear and non-linear systems to stochastic dynamic loading. Mathematical modelling techniques are subdivided into the frequency domain based analytical methods and time domain based numerical methods and each is reviewed for its merits and limitations. Following the description of the numerical methods, which require sample functions of the input stochastic process (i.e. earthquake) and a hysteresis model to describe the inelastic restoring force in the dynamic equation of motion, earthquake ground motion models and hysteresis models are reviewed. Chapter 2 concludes with a summary of past seismic structural response and reliability studies. Chapter 3 summarizes the reasons for choosing the numerical approach in modeling the probabilistic response of structural systems and details the development of the required numerical algorithms. Computational issues associated with time-history analysis, hysteresis modeling, earthquake generation and filtering, random number generation and solution of the structural dynamics eigenvalue problem are discussed. Chapter 4 outlines the basic framework of the software application that was developed, tentatively titled PSResponse, 
along with a description of the structure of the user interface overlaying the computational framework. Following the description of the overall architecture of the software, each of the key features available to the user and the appearance of the user interface are illustrated with screen captures taken from each type of dynamic analysis. The screen captures are accompanied in most cases by an explanation of the required and optional user inputs. Chapter 5 summarizes the verification process that was carried out to establish the accuracy of the software and presents two case studies that were done to demonstrate the capabilities of the program as a research and analysis tool. Finally, Chapter 6 summarizes the development of PSResponse, summarizes the conclusions that were drawn from the case studies of Chapter 5 and discusses possible future developments for the software. 


\section{CHAPTER 2}

\section{LITERATURE REVIEW}

\section{$2.1 \quad$ INTRODUCTION}

The application of probabilistic methods in the field of structural dynamics has followed from the original engineering application of these methods in the aerospace industry for the study of random phenomena such as; engine noise related acoustic fatigue failure, fluctuating airframe stresses associated with wind gusts, and landing gear stresses (Lin 1967). Probabilistic methods and their underlying theory originated in the initial work of physicists on the theory of Brownian motion, which was developed in the early years of the $20^{\text {th }}$ century. Since that time, researchers in the fields of physics, engineering and mathematics have expanded the theory and application of probabilistic methods into a vast field of its own that crosses back and forth over traditional discipline boundaries.

Section 2.2 of this chapter summarizes the key research related to the mathematical modelling of probabilistic structural behaviour that has been carried out over the past 50 years. A complete listing of all relevant research was not attempted due to the sheer volume of work that has been done on the subject. Rather, attention was focused on highlighting the work that most directly formed the background for further research in each area up to the present time.

Sections 2.3 and 2.4 summarize the models that have been developed by researchers for the generation of artificial ground motion time-histories and representation of a non-linear, hysteretic restoring force in the dynamic equation of motion. These models are not included in Section 2.2 since they are not probabilistic 
methods per se, instead they are a necessary component of the numerical methods detailed in Section 2.2 .

The final section of this chapter briefly reviews past seismic structural response and reliability studies that have utilized one or several of the mathematical modeling techniques summarized in Sections $2.2-2.4$.

\subsection{MATHEMATICAL MODELLING OF STRUCTURAL BEHAVIOUR}

The unpredictable nature of earthquakes in their arrival time, magnitude, duration and frequency content means that the dynamic loading on a structure cannot be described by a deterministic function of time. Seismic loading can only be defined in a statistical sense, a so-called random excitation, and the resulting structural response to this loading is also a random function of time. The analysis of structural response to random vibration is termed stochastic dynamic analysis, or more commonly, random vibration analysis.

In the event that seismic structural response stays within the linear-elastic range, as in the case of small earthquakes, the structural restoring force is easy to model and the response statistics or probability densities can be obtained by well-developed linear random vibration methods in the frequency domain. However, to study the performance of structures under severe ground motion, where inelastic response behaviour occurs as a result of plastic deformation of structural elements and connections, linear random vibration methods no longer apply and the changing nature of the structural restoring force needs to be taken into consideration. It is well known that when a structure becomes inelastic the restoring force becomes highly non-linear and hysteretic whereby it depends on the prior history of motion of the system and whether the deformation is increasing or decreasing. In addition, the restoring force may deteriorate in strength or stiffness, or both, as the random vibration progresses. With this reality in mind, the modeling of the restoring force and the subsequent response analysis of an inelastic 
structure to random vibration is a difficult problem for which very few exact solutions, in the probabilistic sense, exist.

The various analytical and numerical methods that have been developed to predict the response of non-linear systems under stochastic dynamic loading are reviewed in the following sections. The merits and limitations of each method are included for the purpose of identifying which one may best suit the modeling of civil engineering structures.

\subsubsection{Analytical Methods}

Analytical procedures are all termed random vibration methods. As in the case of linear elastic systems, the analysis is carried out in the frequency domain, which, at least in principle, yields the complete response statistics to a random loading.

\subsubsection{Markov Methods Based on the Fokker-Planck-Kolmogorov Equation}

\subsection{General Random Vibration Theory and Markov Process Theory}

A Markov stochastic process is termed a one-step-memory random process since it has the property that its present state is only dependent on its immediate past state (Lin 1967). For a discrete random process $X(t)$, this property is expressed in the following relationship between the conditional probability functions:

$$
p\left(x_{n}, t_{n} \mid x_{n-1}, t_{n-1} ; \ldots . ; x_{2}, t_{2} ; x_{1}, t_{1}\right)=p\left(x_{n}, t_{n} \mid x_{n-1}, t_{n-1}\right) t_{n}>t_{n-1}>\ldots \ldots t_{2}>t_{1}
$$

The conditional probability function appearing on the right-hand side of [1] is called the transition probability function of the Markov process $X(t)$. A discrete Markov process, usually called a Markov chain, is completely defined by its first probability function $p\left(x_{1}\right.$, $t_{l}$ ) and the transition probability function. If the initial state of a Markov process is known, $p\left(X_{0}=x_{0}\right)=1$, which is a common situation in many practical applications, the process is then completely characterized by its transition probability. 
In the case of structural dynamics, where random processes are continuously valued, the transition probability function is called the transition probability density and analogous to the discrete case, a continuous Markov process, which is sometimes called a diffusion process, is specified in any one of three equivalent ways:

1. by the first probability density $p\left(x_{1}, t_{1}\right)$ and the transition probability density $p\left(x_{2}\right.$, $\left.t_{2} \mid x_{1}, t_{l}\right)$

2. by the second probability density $p\left(x_{1}, t_{l} ; x_{2}, t_{2}\right)=p\left(x_{1}, t_{l}\right) p\left(x_{2}, t_{2} \mid x_{1}, t_{l}\right)$

3. by the transition probability density if the initial value of the random process is known at least with probability 1.

The transition probability density of a Markov process is governed by an integral equation that is given as follows. For an arbitrary continuous scalar random process,

$$
\begin{aligned}
p\left(x_{2}, t_{2} \mid x_{1}, t_{1}\right) & =\int p\left(x_{2}, t_{2} ; x, t \mid x_{1}, t_{1}\right) d x \\
& =\int p\left(x, t \mid x_{1}, t_{1}\right) p\left(x_{2}, t_{2} \mid x, t ; x_{1}, t_{1}\right) d x
\end{aligned}
$$

However, if $X(t)$ is Markovian and the transition probability density is denoted by $q_{x}$, Eq. [2] reduces to:

$$
q_{x}\left(x_{2}, t_{2} \mid x_{1}, t_{1}\right)=\int q_{x}\left(x, t \mid x_{1}, t_{1}\right) q_{x}\left(x_{2}, t_{2} \mid x, t\right) d x
$$

Equation [3] is known as the Chapman-Kolmogorov-Smoluchowski equation for a onedimensional random process and it is readily extended to a vector random process. Solutions to this integral equation, whether scalar or vector-valued, are frequently obtained by solving an equivalent partial differential equation called the Fokker-PlanckKolmogorov (FPK) equation that describes the evolution of the transition probability density function. For a one-dimensional Markov process this equation is given by: 


$$
\frac{\partial}{\partial t} q_{x}+\frac{\partial}{\partial x}\left(A q_{x}\right)-\frac{1}{2 !} \frac{\partial^{2}}{\partial x^{2}}\left(B q_{x}\right)+\frac{1}{3 !} \frac{\partial^{3}}{\partial x^{3}}\left(C q_{x}\right)-\frac{1}{4 !} \frac{\partial^{4}}{\partial x^{4}}\left(D q_{x}\right)+\ldots . .=0
$$

where the coefficients $A, B, C, D$ etc. are called the derivative moments, which give the rates of various moments of the increment in $X(t)$ conditional on $X(t)=x$. The derivative moments can be written as:

$$
\begin{aligned}
& A=A(x, t)=\lim _{\Delta t \rightarrow 0} \frac{1}{\Delta t} E[X(t+\Delta t)-X(t) \mid X(t)=x] \\
& B=B(x, t)=\lim _{\Delta t \rightarrow 0} \frac{1}{\Delta t} E\left\{[X(t+\Delta t)-X(t)]^{2} \mid X(t)=x\right\} \\
& C=C(x, t)=\lim _{\Delta t \rightarrow 0} \frac{1}{\Delta t} E\left\{[X(t+\Delta t)-X(t)]^{3} \mid X(t)=x\right\} \\
& D=D(x, t)=\lim _{\Delta t \rightarrow 0} \frac{1}{\Delta t} E\left\{[X(t+\Delta t)-X(t)]^{4} \mid X(t)=x\right\}
\end{aligned}
$$

The FPK equation for the transition probability density of a Markov vector random process is identical except that each scalar transition probability density $q_{x}$ is replaced by the vector transition probability density $q_{\{x\}}$ with the number of dimensions equal to the number of state variables in the underlying stochastic process. The FPK equation is also known as the Kolmogorov forward equation where the adjective 'forward' refers to the fact that the time derivative in the equation is a derivative with respect to the later time. There is a corresponding Kolmogorov backward equation, which is the adjoint of the forward equation, where the time derivative is with respect to the earlier time.

It has been shown (Lin 1967) that the response vector of a non-linear system, whether single degree of freedom or multi-degree of freedom, under the excitation of a shot-noise or a filtered shot-noise is a Markov vector process. A shot-noise $S(t)$ is a random process in which the mean and auto-covariance functions are given by: 


$$
\begin{aligned}
& \mu_{S}(t)=E[S(t)]=0 \\
& \kappa_{S S}\left(t_{1}, t_{2}\right)=I(t) \delta(\tau) \\
& \kappa_{S S}\left(t_{1}, t_{2}\right)=\kappa_{2}\left[S\left(t_{1}\right), S\left(t_{2}\right)\right]=E\left\{\left[S\left(t_{1}\right)-\mu_{S}\left(t_{1}\right)\right]\left[S\left(t_{2}\right)-\mu_{S}\left(t_{2}\right)\right]\right\} \\
& \kappa_{S S}\left(t_{1}, t_{2}\right)=R_{S S}\left(t_{1}, t_{2}\right)-\mu_{S}\left(t_{1}\right) \mu_{S}\left(t_{2}\right) \\
& R_{S S}\left(t_{1}, t_{2}\right)=E[S(t) S(t+\tau)]
\end{aligned}
$$

where $\kappa_{2}$ is the second cumulant function, which is equivalent to the second central moment function, $R_{S S}$ is the auto-correlation function which in the case of a zero mean process such as a shot-noise is equivalent to the auto-covariance function, $I(t)$ is the intensity function of the shot-noise and $\delta(\tau)$ is the Dirac delta function. In the case of a weakly stationary shot-noise, which by definition has a mean function that is independent of time and a correlation function that is dependent only on the time difference $\tau=t_{1}-t_{2}$, the intensity function is time invariant and therefore constant which results in the auto-correlation function being an impulse at time $t=\tau$. The spectral density of the weakly stationary shot-noise, which by the well-known WienerKhintchine theorem is the Fourier transform of the auto-correlation function $R_{S S}(\tau)$, is then given by:

$$
\begin{aligned}
& \Phi_{s S}(\omega)=\frac{1}{\pi} \int_{0}^{\infty} R_{S S}(\tau) \cos (\omega \tau) d \tau \\
& \Phi_{s S}(\omega)=\frac{I}{\pi} \int_{0}^{\infty} \delta(\tau) \cos (\omega \tau) d \tau \\
& \Phi_{s S}(\omega)=\frac{I}{2 \pi}
\end{aligned}
$$

Therefore, the spectral density is constant. A weakly stationary random process with a constant spectral density is called a white-noise. The physical interpretation of a constant spectral density is that the energy content in the random process is uniformly distributed over the entire frequency range. The mean-square value of a random process, which is a measure of the average energy content, is given by: 


$$
E\left[x^{2}(t)\right]=\overline{x^{2}}=\lim _{t \rightarrow \infty} \frac{1}{t} \int_{0}^{t} x^{2} d t=R_{X X}(0)
$$

also

$$
\begin{aligned}
& R_{X X}(\tau)=2 \int_{0}^{\infty} \Phi_{X X}(\omega) \cos (\omega \tau) d \omega \\
& R_{X X}(0)=2 \int_{0}^{\infty} \Phi_{X X}(\omega) d \omega
\end{aligned}
$$

and

$\sigma^{2}=\overline{x^{2}}-(\bar{x})^{2}$

Therefore, the mean-square value of a white-noise, which is the area under the spectral density curve $\Phi_{\mathrm{XX}}(\omega)$, is equivalent to the variance of the zero mean process and is unbounded. Since a physically realizable random process cannot have an infinite average energy the white-noise process is a mathematical idealization. Similarly, non-stationary shot-noise and filtered shot-noise, which is shot-noise passed through an appropriate linear filter, are also mathematical idealizations and, strictly speaking, physically impossible. However, response analysis in which the excitation is modeled as nonstationary shot-noise, filtered shot-noise or white-noise, leading to a Markovian response vector $\{Z, Z Z$, which represents the random displacement $Z(t)$ and random velocity $\dot{Z}(t)$ of each degree of freedom, can give meaningful results.

To determine whether or not the mean-square output of a system computed by approximating an actual excitation spectral density by a white-noise spectral density is a good approximation to the actual mean-square output, a single-degree-of-freedom linear structure will be considered. Classical linear random vibration theory states:

$$
S_{Y}(\omega)=H(\omega) \cdot H^{*}(\omega) \cdot S_{X}(\omega)=|H(\omega)|^{2} \cdot S_{X}(\omega)
$$

where $S_{Y}(\omega)$ is the output spectral density, $S_{X}(\omega)$ is the input spectral density and $|H(\omega)|^{2}$ is the system transfer function or transmittancy function or frequency response function. In the case of an SDOF system, the system transfer function is given by: 


$$
|H(\omega)|^{2}=\frac{1}{m^{2}\left[\left(\omega_{0}^{2}-\omega^{2}\right)^{2}+\left(2 \xi \omega_{0} \omega\right)^{2}\right]}
$$

For a white-noise input, where the spectral density is a constant $=\mathrm{S}_{0}$, the mean-square output is then given by:

$$
E\left[Y^{2}\right]=\int_{-\infty}^{\infty} S_{Y}(\omega) d \omega=\int|H(\omega)|^{2} S_{X}(\omega) d \omega=R_{Y Y}(0)=\frac{\pi \cdot S_{0}}{2 \xi \omega_{0}{ }^{3} m^{2}}
$$

Therefore, the mean-square output of a damped system $(\xi>0)$ is finite even when the mean-square input is infinite as is the case for an ideal white-noise. Also, for a lightly damped system, which is applicable to most practical civil engineering structures, the transfer function is sharply peaked at the undamped natural frequency, $\omega_{0}$, of the structure and the system acts like a narrow-band filter. Therefore, the major contribution to the integral in Eq. [11] is obtained in the vicinity of the natural frequency of the structure and the value of the input spectral density, $S_{X}(\omega)$, outside that vicinity is unimportant. Assuming the spectral density of the actual excitation is slowly varying in the vicinity of the structure's natural frequency, it may be reasonably approximated by a white-noise spectral density (see Fig. 2.1).

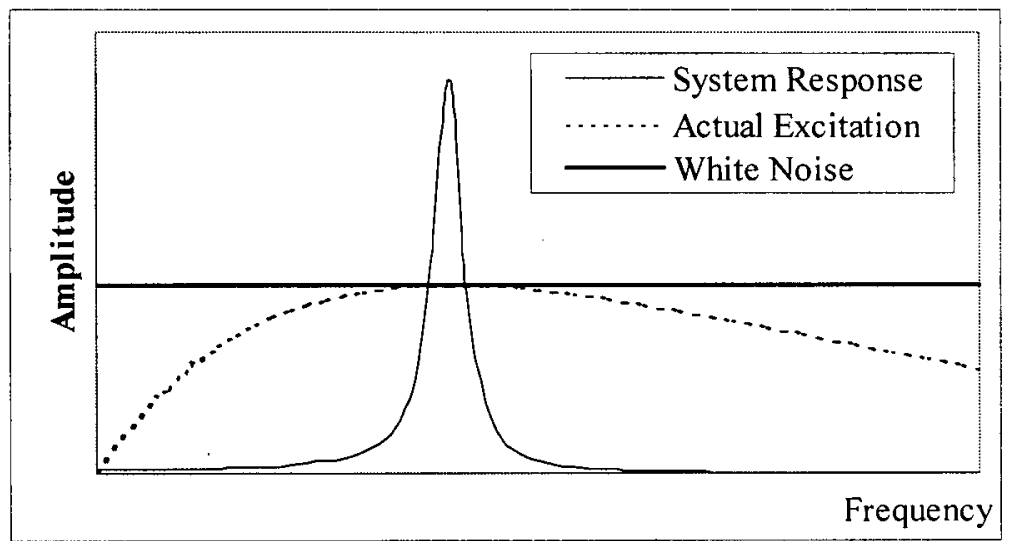

Figure 2.1: System Response and White Noise Approximation 
For the assumption of a non-stationary shot noise as an approximation to the actual excitation it is not possible to state in mathematical terms the conditions under which the approximation is acceptable, as was done in the case of the white-noise idealization. The case of a non-stationary shot-noise, however, is relevant particularly in seismic response analysis since earthquake ground motions are non-stationary random processes. The non-stationary nature of earthquakes is clearly evident in a typical acceleration-time record, which shows a general trend of first increasing and then decreasing in intensity with time. Also, the correlation in the ground motion for two time instants, say, one second apart is clearly not constant throughout the record. A stationary process by definition has an auto-correlation function which is constant for a given time separation $\tau$. If the non-stationary shot-noise excitation is modeled as a sequence of random impulses with independent arrival times (i.e. Poisson distributed with a nonstationary arrival rate) and independent amplitudes, then the characteristics of the structure determine whether it will respond in the same manner to the random impulses as under a real earthquake. The effect would be approximately the same if the average time spacing between independent impulses substituting for the actual earthquake record were 'short' as sensed by the structure. The response of a single-degree-of-freedom structure to an impulse has the form of damped free vibration, so a useful measure of the 'shortness' of the average time spacing between independent impulses is to compare that spacing with the damped natural period of vibration of the structure. The assumption that a real earthquake can be modeled as non-stationary shot noise will be valid if the damped natural period is, say, an order of magnitude longer than the average time spacing between impulses.

When the damped natural period of a structure is not much longer than the time separation for which the earthquake record is essentially uncorrelated, a model more general than non-stationary shot-noise is required to model the earthquake ground motion. In this case, however, the response of the structure to the excitation is no longer a Markov vector process. With this understanding, the superposition of random pulses with an assumed pulse shape and independent arrival times and amplitudes can be used in this situation and the general theory of random pulses can then be applied. It should be 
noted that shot-noise is simply a special case of the superposition of random pulses in which the pulse shape is given by the impulse function $\delta(t-\tau)$. More detail regarding the modeling of earthquake ground motions using random pulses is given in Section 2.3. Using random pulse theory, the variance function of a random pulse train with a sinewave pulse shape is shown to increase with time to a maximum and then decrease with time to zero. This shows that the average energy in the simulated excitation increases and then decreases with time in the same manner as a typical earthquake record. From this evidence it is concluded that random pulse trains can be used to model earthquakes in the event that non-stationary shot-noise is not a suitable model due to the short damped natural period of a given structure. In this case, however, the response of the structure is no longer Markovian.

Given that an actual excitation can reasonably be modeled as a shot-noise, either stationary or non-stationary, the response of a non-linear system is a Markov vector process, as was noted previously. In the case of a single degree of freedom non-linear system, the governing differential equation is given by:

$$
\ddot{Z}+g(Z, \dot{Z})=F(t)
$$

where $g$ is a non-linear function of the displacement $Z$ and velocity $\dot{Z}$. Let $Z=Y_{I}$ and $\dot{Z}$ $=Y_{2}$, the differential equation of motion is then equivalent to two first-order equations given by:

$$
\begin{aligned}
& \dot{Y}_{1}=Y_{2} \\
& \dot{Y}_{2}=-g\left(Y_{1}, Y_{2}\right)+S(t)
\end{aligned}
$$

where the shot-noise $S(t)$ has replaced $F(t)$. If $S(t)$ is Gaussian, then the FPK equation governing the transition probability density $\mathrm{q}_{\{\mathrm{Y}\}}\left(\mathrm{Y}, \mathrm{t} \mid \mathrm{Y}_{0}, \mathrm{t}_{0}\right)$ is given by:

$$
\frac{\partial q}{\partial t}+Y_{2} \frac{\partial q}{\partial Y_{1}}-\frac{\partial}{\partial Y_{2}}\left[q g\left(Y_{1}, Y_{2}\right)\right]-\frac{I(t)}{2} \frac{\partial^{2} q}{\partial Y_{2}^{2}}=0
$$


where $I(t)$ is the intensity function of the shot-noise. A Gaussian or normal process is a random process whose probability distribution is completely defined by its mean function, $\mu_{X}(\mathrm{t})$, and its covariance function, $\kappa_{X X}(\mathrm{t})$. Equivalently, a Gaussian random process may be defined as one in which the cumulant functions higher than second order are equal to zero. With this property, a weakly stationary Gaussian process is also strongly stationary since all moments higher than second order are zero. The assumption of a Gaussian random process is frequently used in random vibration analysis since many real phenomena can be satisfactorily modeled using that assumption. This is a consequence of the Central Limit Theorem, which states that when a random process is the sum of a large number of independent random processes, it approaches a normal process, regardless of the distribution of the individual constituents, as the number of independent constituents increases without limit. In the case of a shot-noise, the process tends to a Gaussian distribution as the arrival rate of the impulses tends to infinity. Unfortunately, even with the assumption of a Gaussian process, which is further limited to the assumption of a white-noise process, no exact solution has been found for the FPK equation, Eq. [14].

\subsection{Solution Methods for the Fokker-Planck-Kolmogorov Equation}

A number of solution techniques have been developed to approximate the transition probability density function in the FPK equation. The assumption of a stationary response, in which the first term in the equation is neglected, was initially used (Caughey 1963) to develop an analytical solution for the joint probability density between the stationary displacement and velocity of a single-degree-of-freedom system. However, this method relied on a non-hysteretic assumption for the non-linear stiffness function and the stationary assumption was too restrictive in scope for short-term transient processes. For this reason, a number of more general numerical methods were developed to solve the FPK equation, some of which are briefly summarized below. 


\subsection{Galerkin Based Methods}

The Galerkin method is one of the weighted residual methods that are used for approximating the solution to a differential equation governing the behaviour of a continuous system (Bathe 1996). The Galerkin method uses trial functions that are identical with the weighting functions and the integral of the product of the weighting functions and the residual is set to zero to determine the coefficients for each trial function. Stratonovitch (1964) and Atkinson (1973) used eigenfunctions of the FPK equation of a linear system as trial functions while Bhandari and Sherrer (1968) used Hermite polynomials as trial functions to represent the transition probability density function under a white-noise excitation. In each case the solution assumed a stationary response for a weakly non-linear one or two-degree-of-freedom system, for which the associated FPK equation is of two and four dimensions respectively. Wen (1975) extended these results using Hermite polynomial trial functions to include the transient response using a filtered Gaussian shot-noise to take the non-stationarity and spectral content of the excitation into consideration. Later Wen (1976) extended his results to hysteretic systems using a smooth differential equation model for hysteresis first proposed by Bouc (1963) that was compatible with the Markov-Galerkin formulation of the FPK equation (see Sec. 2.4).

The disadvantage of the Galerkin based approach is the low rate of convergence for highly non-linear systems and the complexity of the integrals involved in cases where the non-linearities do not take the form of polynomials. To improve the convergence

rate for this type of solution of the FPK equation, Soize (1988) proposed a method that allowed for the steady-state solution of systems of higher order, say, 10 to 20.

\subsection{Finite Element Method}

The Finite Element Method can be regarded as an extension of the classical weighted residual methods. Using a Finite Element solution method for the stationary FPK equation, Langley (1985) and Langtangen (1991) integrated the weighted residual 
statement for the problem to produce the weak form of the FPK equations and then chose weighting (or shape) functions defined for finite regions (or elements) of the problem domain. In this way, a set of linear equations was constructed in which the unknowns are the values of the joint probability density function at a number of points (or nodes) in the domain. These equations are then solved by standard matrix methods to yield the stationary transition probability density function of the response process.

Spencer and Bergman (1985) developed a Finite Element algorithm to solve the FPK equation for smooth hysteretic stationary systems and then later applied the algorithm to the transient FPK equation and obtained a solution for the evolution of the transition probability density function for two classical non-linear second order oscillators, the Duffing Oscillator and the Van der Pol Oscillator, subjected to an additive white-noise excitation (Bergman and Spencer 1991). An external excitation, which in general is represented by $g(Z, \dot{Z}) \cdot F(t)$, is characterized as additive if the accompanying coefficient $g(Z, \dot{Z})$ is just a constant, whereas a multiplicative excitation is displacement and/or velocity dependent resulting in a non-constant coefficient $g(Z, \dot{Z})$.

The drawback of the Finite Element solution method is that in practice it is limited to single-degree-of-freedom systems due to the high computational effort required for systems of higher than two dimensions. Also, as with all FPK equation based solution methods, the excitation is assumed to be a stationary Gaussian process (i.e. white-noise).

\subsection{Closure Techniques}

The problem of closure arises frequently when analyzing the response of a nonlinear system to random excitation. The differential equations that describe certain moments of the non-stationary response contain higher moments and when additional equations for the higher moments are derived they contain even higher moments. A closure technique refers to a procedure by which the infinite hierarchy of differential equations governing the statistical moments of a random vibration response process is 
truncated at some order. The higher order terms in the remaining lower order moments are then expressed in terms of the lower order moments. In the case of a stationary response, the differential equations reduce to a set of algebraic equations.

The derivation of the infinite hierarchy of differential equations for moments or cumulants of the response process utilizes the FPK equation in terms of the Itô type equation. Itô (1951) showed that a Markov diffusion process $X(t)$ is governed by a stochastic differential equation given by:

$$
d X(t)=\mu(x, t) d t+\sigma(x, t) d W(t)
$$

where $\mu$ and $\sigma$ are the drift and diffusion coefficients of the process and $W(t)$ is a unit Wiener process describing Brownian motion, which is a Gaussian random unit $(\sigma=1)$ process with stationary independent increments and is therefore a Markov process. Equation [15] describes the motion of a mechanical system under random (white-noise) excitation and leads directly to the FPK equation through the use of the time derivative of the moment generating function of the response process $X(t)$ (Sólnes 1997). The associated infinite hierarchy of differential equations governing the statistical cumulants of $X(t)$, are given by (Wu 1987):

$$
\begin{aligned}
& \frac{d \kappa_{1}(t)}{d t}=E[\mu(x, t)] \\
& \frac{d \kappa_{2}(t)}{d t}=2 E\left[\left(x-\kappa_{1}\right) \mu(x, t)\right]+E\left[\sigma^{2}(x, t)\right] \\
& \frac{d \kappa_{3}(t)}{d t}=3 E\left[\mu(x, t)\left[\left(x-\kappa_{2}\right)^{2}-\kappa_{2}\right]+3 E\left[\left(x-\kappa_{1}\right) \sigma^{2}(x, t)\right]\right. \\
& \frac{d \kappa_{4}(t)}{d t}=\ldots \ldots \ldots \ldots . . .
\end{aligned}
$$

As stated previously, in the case of a stationary response, $d \kappa_{n} / d t=0$, and Eqs. [16] reduces to a set of algebraic equations. 
The simplest closure scheme for solving Eq. [16] is Gaussian Closure in which the expressions of the order $>2$ are expressed in terms of the first two cumulants and then the first two equations are solved. Iyengar (1978) used Gaussian Closure to study the response of a hysteretic system with a smooth restoring force. To improve this closure scheme, non-Gaussian properties have been considered either in terms of approximating the unknown probability density with a truncated Gram-Charlier or Edgeworth series (Crandall 1980), which are general purpose probability distributions, or by including addition cumulants of higher order than 2 (Wu and Lin 1984). Suzuki and Minai (1987) utilized a non-Gaussian closure technique to thoroughly analyze the response characteristics of inelastic systems including displacement, velocity, maximum response, cumulative plastic deformation and low cycle fatigue damage factor. Their procedure used a series expansion of the joint probability density function of the response state vector in terms of a product of normal gamma density and orthogonal polynomials. The state vector included specified quantities concerned with the white-noise shaping filter for seismic excitation, the hysteretic structure and also with measures of structural damage.

Although closure techniques apply to transient non-stationary response processes and also to systems with multiple degrees of freedom, the major shortcoming of this method is the significant increase in computational effort required for a modest increase in the number of degrees of freedom of the system as well as the restriction of the excitation to a white-noise process.

\subsection{Stochastic Averaging}

The principle of Stochastic Averaging is to simplify the equations describing slowly fluctuating response quantities by time-averaging the rapidly fluctuating response quantities. This principle is a non-trivial extension of the Krylov-Bogoliubov averaging method for deterministic excitations since it involves accounting for the averaged effect of a random excitation multiplied by a correlated response (Zhu 1988). Stochastic Averaging Methods in random vibration analysis can be viewed as a combination of this 
Stochastic Averaging principle and the FPK equation method. By averaging certain response quantities, the FPK equation is simplified or even reduced in dimension, which reduces the difficulties in solving it.

There are three methods of Stochastic Averaging, namely, the Standard Stochastic Averaging Method, the averaging method of coefficients in the FPK equation, and the Generalized Stochastic Averaging Method. The standard method was developed first by Stratonovitch (1964) and it applies to narrow-band responses, which are represented as sinusoidal oscillations with slowly varying amplitude and phase. Approximate equations for the slowly varying quantities are obtained by time-averaging the rapid fluctuations. In the second method, developed by Khasminskii (1963), the drift and diffusion coefficients in the FPK equation are averaged with respect to time. In the third method, also developed by Stratonovitch (1964) and alternatively known as the Stochastic Averaging Method of the energy envelope, the response variables are divided into rapidly varying quantities and slowly varying quantities and approximate equations for the latter are obtained by averaging the rapid fluctuations of the former. For a single-degree--offreedom system the rapidly varying quantity is the displacement and the slowly varying quantity is the energy envelope.

The application of the Stochastic Averaging Methods, which were first developed to analyze non-linear phenomena in radio engineering, to mechanical and structural systems began in the late 1970's for predicting the response, deciding the stability and estimating the reliability of non-linear systems subject to random external and parametric excitation. A parametrically excited system is one in which the effective stiffness and/or damping parameters are forced to vary with time. The most important property of such systems is that for periodic parametric excitation there are ranges of excitation amplitude and frequency for which the response remains bounded (stable) and ranges for which the response grows without limit (unstable). This type of excitation, however, is not relevant to the analysis of civil engineering structures and will not be considered further. In the case of externally excited structures, the central idea of the Stochastic Averaging Methods is that if the typical structural response time is much longer than the excitation 
correlation time, the excitation in effect acts as independent pulses and the response of the system can be described in terms of a scalar quantity, usually the energy content of the system, which is approximately a one-dimensional Markov process. Therefore, the dimension of the problem is greatly reduced and the solution of the FPK equations becomes much simpler. For a single-degree-of-freedom narrow-band (lightly damped) structural system subjected to a wide-band excitation, the Standard Stochastic Averaging Method may be used to transform the state vector $\{Z, \dot{Z}\}$ to a pair of slowly varying processes expressed in terms of the amplitude $a(t)$ and phase angle $\varphi(t)$ as follows:

$$
\begin{aligned}
& Z(t)=a(t) \cos (\omega t+\varphi(a, t)) \\
& \dot{Z}(t)=-\omega a(t) \sin (\omega t+\varphi(a, t))
\end{aligned}
$$

By time-averaging over the period of oscillation the phase angle $\varphi$ can be eliminated i.e. uncoupled from the FPK equation, which results in $a(t)$ being a one dimensional Markov process. Therefore, as stated previously, the dimension of the problem is reduced and the solution of the FPK equation becomes much simpler.

Iwan and Lutes (1968) applied Stochastic Averaging Methods to non-linear systems with bilinear hysteresis and showed that it gives inaccurate results for systems with large non-linearities. However, it was later shown that the Krylov-Bogoliubov technique, which forms the basis of the Stochastic Averaging Method, may seriously overestimate the energy dissipation capacity of elasto-plastic or nearly elasto-plastic systems (Wen 1980). This results in a large underestimation of the root-mean-square (RMS) response of the system in a certain response range. Later, Roberts (1978) came to the conclusion that Stochastic Averaging was applicable to an oscillator with a bilinear restoring force-displacement characteristic. Extending that work, Roberts and Spanos (1986) applied Stochastic Averaging Methods to smooth hysteretic systems and obtained good results for narrow-band systems. Cai and Lin (1988) used a similar approach, which was applicable to either bilinear or smooth hysteretic systems, without the restriction that the response be a narrow-band process. In this approach, which was termed Equivalent Non-linearization, the original system that cannot be solved exactly is 
replaced by a substitute non-linear system for which an exact solution is known. To find the best non-linear approximation, the mean-square error is minimized. The application of this procedure is quite restricted since exact analytical solutions exist only for a very limited class of problems, most of which are not applicable to practical engineering cases.

Further developments in applying averaging techniques to randomly excited systems have been given by several authors using both Stochastic Averaging and QuasiConservative Averaging Methods. Quasi-conservative averaging was originally developed by Landa and Stratonovitch (1962) and Khasminskii (1964) in which the equation of motion of a system is replaced two first-order equations for energy and displacement. The original method, which was only applicable under Gaussian whitenoise, was extended by Roberts (1982) and others to include non-white additive excitation and then further extended by Cai (1995) using Roberts' scheme to include multiplicative excitations. Lin and Cai (2000) recently addressed the problem of multiple-degree-of-freedom systems with both high and low damping modes, and/or strongly non-linear stiffness under non-white stochastic additive and multiplicative excitation.

\subsection{Numerical Diffusion Techniques}

Numerical Diffusion Techniques, also known as Cell Mapping Methods, were developed from the theory of point-to-point mapping of dynamic systems attributed to Poincare in the $19^{\text {th }}$ century. For a dynamic system governed by:

$$
\dot{z}(t)=F(t, z(t))
$$

where $z$ is a real-valued $n$-dimensional vector and $F$ is a real-valued vector function that is explicitly periodic in $t$, the governing equation may be integrated over one period to relate the state of the system at the end of one period to the state at the end of the next period. Viewed in this manner, the governing equation for the system takes on the form: 


$$
z(n+1)=G(z(n))
$$

in which a point $z(n)$ in the state space, or phase plane $\{Z, \dot{Z}\}$, is mapped by $G$ after one period into a point $z(n+1)$. Such a point-to-point mapping dynamic system is called a point map or a Poincare map in the mathematical literature. A Poincare map is a mathematical idealization of the dynamic system since, considering physical limitations on measurement accuracy and the inherent round-off error in numerical evaluation, there is a limit beyond which two values of a state variable cannot be differentiated and therefore must be treated as the same. For this reason the state variables must be treated as having discrete values which leads to the idea of considering the state space not as a continuum of points but rather as a collection of very small intervals or cells. The theory of point-to-point mapping then becomes one of cell-to-cell mapping, which can be used to study the global behaviour of real non-linear dynamic systems governed by ordinary differential equations. Cell Mapping Methods, in the context of analyzing dynamic systems through discretization, may be viewed as discretizing the dependent state variables, whereas classical stepwise time integration is a procedure to discretize the independent time variable and Finite Element analysis is a procedure to discretize the independent spatial variables.

Hsu (1980) and Hsu and Guttalu (1980) developed an algorithm for analyzing the behaviour of non-linear dynamic systems using cell-to-cell mapping, which was termed simple cell mapping, since each cell could only be mapped to one other cell, called an image cell, during each iteration of the algorithm. This work was later extended to a generalized cell mapping (GCM) algorithm (Hsu 1981, Hsu et al. 1982) which allowed for the mapping of a cell to multiple image cells, each image cell possessing a fraction of the total probability of occurrence. The probabilities $P_{i j}$ of mapping cell $i$ to cell $j$ in one mapping step are contained in a transition probability matrix $\boldsymbol{P}$, which completely controls the evolution process of the dynamic system. Using the theory of discrete Markov chains and knowing the initial state of the dynamic system, the probability of the system being in a given future state (cell) after $n$ mapping steps is completely determined. 
This can be shown by using the discrete form of the Chapman-KolmogorovSmoluchowski equation, Eq. [3], which is given by:

$$
P_{i, j}^{m+n}=\sum_{k} P_{i, k}^{m} \cdot P_{k, j}^{n} \quad \cdot 0 \leq m \leq n
$$

where the $n$-step transition probability $P_{k o j}^{n}$ is defined as the probability of being in cell $k$ after $n$ steps starting from cell $j$. Given in the form of function mapping, Eq. [20] is rewritten as:

$$
P_{i}(n)=\sum_{j=1}^{\infty} P_{i j} p_{j}(n-1), \rightarrow n=1,2 \ldots
$$

where $P_{i}(n)$ represents the probability of the system being in the $i$ th cell at time $n \tau$ and $P_{i j}$ the probability of the system being in the $i$ th cell at time $\tau$ when the system is initially in the $j$ th cell with probability one. Unfortunately, for non-linear stochastic systems, the one-step transition probability matrix $P_{i j}$ involving all $i$ 's and $j$ 's is rarely available and simulation methods are normally required to determine the conditional probability density function of the response process. Hsu and Chiu (1986) used Monte Carlo simulation to construct a histogram estimator of $P_{i j}$ by generating a large number of sample trajectories of time duration $\tau$ out of each cell in the phase plane. However, when a large number of cells are used and a large number of sample trajectories are simulated out of each cell, the Monte Carlo method becomes quite computationally intensive. To avoid the time-consuming simulation of $P_{i j}$, Sun and Hsu (1990) proposed a Gaussian approximation for the conditional probability density function of moving from $j$ th cell to the $i$ th cell when the time $\tau$ is sufficiently small. This Gaussian approximation was allowed to vary in shape with the initial starting point (cell $j$ ) to properly capture the global non-linear system behaviour.

The Generalized Cell Mapping Method is widely applicable to many types of systems that are either weakly or strongly non-linear and either lightly or heavily 
damped. It can provide both transient and steady-state solutions of system response and may even be applied to stochastic systems which don't necessarily admit the FPK equation. The restriction on the GCM method is the assumption of a discrete Markov chain of mapping steps, which is based on the system excitation being a white-noise process. Also, the applicability of GCM to degrading hysteretic systems is not known.

\subsection{First-Passage Problem Solution Methods}

For structural reliability calculations the probability distribution of the time to first-passage (exit) of a safe domain for the response process is of considerable interest. This type of problem is commonly referred to as a first-passage problem. There are three different types of safe domains that are commonly used to characterize the firstpassage problem (see Fig. 2.2). In the first case, the safe domain is characterized by a single barrier or threshold level $z=b$, called a type-B barrier, which is typically described quantitatively in terms of the RMS response level. The second case is similar to the first except that the safe domain is characterized by the double barrier $z= \pm b$, called a type- $D$ barrier. In the third case, a passage level for the envelope process $A(t)$ rather than the process $Z(t)$ itself is considered. An envelope-passage level is called a type-E barrier.

For a damped dynamic system subject to random excitation an exact solution to the first-passage problem remains to be found for any of the barrier types and approximate solution methods must be used. There are two analytical solution strategies that are used to obtain approximate statistics for the time to first-passage. The first strategy relies on the special nature of independent random events with exponential distributions while the second strategy employs Markov theory and does not require the assumption of independent events. 


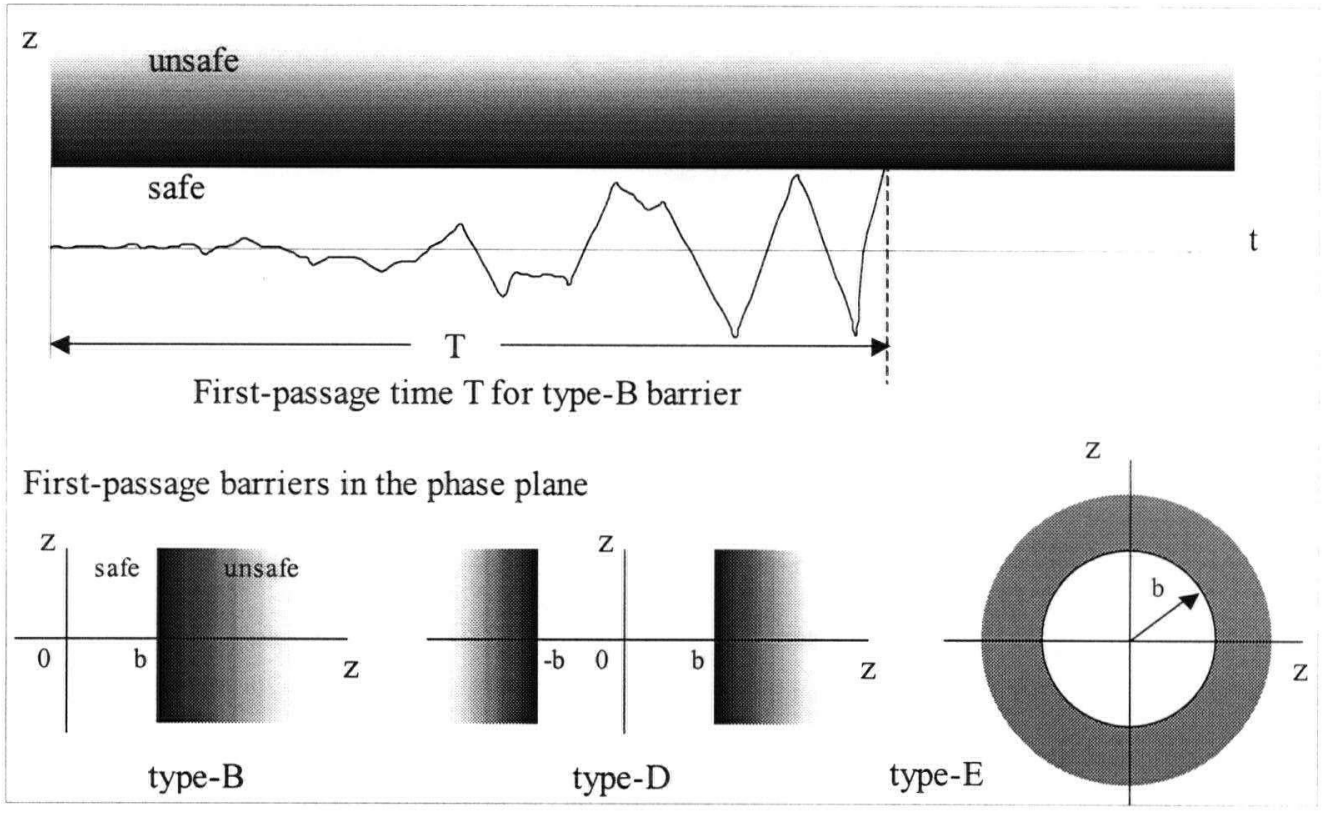

Figure 2.2: First-Passage Time and Domain Barriers in the Phase Plane

\subsection{Assumption of Independent Events}

The assumption of independent events leads to various expressions for the first crossing density, $p_{1}(T)$, which is the density function describing the probability that the response process surpasses a given threshold for the first time (since $t=0$ ) during the interval $T \leq t \leq T+d T$. The form of each expression, assuming a reasonably high threshold, is given by (Crandall 1970):

$$
p_{1}(T)=\alpha e^{-\alpha T}
$$

where $\alpha$ is called the limiting decay rate of the first crossing density, which depends on which type of event is assumed to occur independently. The possible choices are; independent threshold crossings, independent peaks, independent envelope crossings, and independent envelope peaks. 
The simplest approximation to the first crossing density is obtained by assuming independent threshold crossings of type-B or type-D barriers. This is equivalent to assuming that the arrival of failures (i.e. threshold crossings) is rare enough that they can be considered independent events. In this case, the number of failures $n$ within the time interval $[0, t]$ is a Poisson process, which is described by:

$$
P[n, t]=\frac{(v t)^{n}}{n !} e^{-v t}
$$

where $v$ is the mean threshold crossing rate. The mean crossing rate for a type-D barrier is simply twice the mean crossing rate for a type- $\mathrm{B}$ barrier. The probability of no failures $(n=0)$ in the time interval $[0, t]$ is given by:

$$
P[0, t]=e^{-v t}
$$

The probability of failure in the time interval $[0, t]$ is then given by:

$$
P_{F}[0, t]=1-P[0, t]=1-e^{-u t}
$$

This result may be interpreted as the probability that the first-passage time is equal to or less than $t$, which is the cumulative distribution function (CDF) of $T$. The first crossing density then follows from differentiation of Eq. [25]:

$$
p_{1}(T)=v e^{-v t}
$$

Equation [26] can be used to compute the statistical properties of the first-passage time $T$. In particular, the mean and variance of the time until first crossing are given by: 


$$
\begin{aligned}
& E[T]=\int_{0}^{\infty} t p_{1}(t) d t=\frac{1}{v} \\
& \sigma^{2}(T)=\int_{0}^{\infty}(t-E[T])^{2} p_{1}(t) d t=\frac{1}{v^{2}}
\end{aligned}
$$

The threshold crossing rate $v$ is determined from the well known Rice's formula, which for a type-B barrier, is given by:

$$
v=\int_{-\infty}^{\infty}|\dot{z}| f_{Z \dot{z}}(b, \dot{z}) d \dot{z}
$$

where $z=b$ is the threshold level and $f_{z z}$ is the joint density function of $z$ and $z$. When information about threshold crossings only from below $\left(v^{+}\right)$is required, termed the upcrossing rate, the lower limit of integration in Eq. [28] is changed to zero and $v^{+}=1 / 2$ $\nu$. Again, for a type-D barrier the threshold crossing rate and upcrossing rate is simply twice that for a type-B barrier.

In the special case of a stationary Gaussian process with zero mean, the upcrossing rate of threshold $z=b$ is given by:

$$
v^{+}=\frac{1}{2 \pi} \frac{\sigma_{\dot{z}}}{\sigma_{z}} \exp \left(-\frac{b^{2}}{2 \sigma_{z}^{2}}\right)
$$

When the threshold $b$ is zero, the problem is known as the zero-crossing problem, which counts the number of loading cycles. For a stationary normal zero mean process, the expected of rate of zero crossings from below is given by:

$$
v_{0}^{+}=\frac{1}{2 \pi} \frac{\sigma_{\dot{z}}}{\sigma_{Z}}
$$


The assumption of independence of threshold crossings is not well suited to narrow-band processes such as the response of lightly damped dynamic systems. Once a sample function of a narrow-band random process crosses a given threshold level $b$ or $|b|$, the probability is high that the following excursion will produce another crossing. -Therefore, for every threshold crossing of an envelope $A(t)$, there may be several threshold crossings of the narrow--band process $Z(t)$, which is enclosed by the envelope, and consequently, independence of the narrow-band crossings is lost. This phenomenon is referred to as clumping. The importance of taking clumping into consideration is measured by the average clump size for which expressions have been derived by Lyon (1961) and Racicot (1969).

With the clumping phenomenon present, an improved estimate for the first crossing density of a narrow-band random process can be obtained by considering the envelope $A(t)$ and assuming that the envelope crossings of the type-E barrier are independent (Lin 1967). When there are many excursions in each clump, the time of an envelope threshold crossing, which must precede the first crossing in each clump, is nearly the same as the time of the first crossing of the clump. For this reason there is little difference between the results for type-D and type-E barriers. Using the expected rate of threshold crossings of the envelope process, expressions analogous to Eq. [26] and Eqs. [27] are obtained for first crossing density and first-passage time $T$ of the envelope process. For a stationary Gaussian random process, the expected type-E barrier upcrossing rate of the envelope process is given by:

$$
v_{E}^{+}=\frac{b \sigma_{1}}{\sigma^{2} \sqrt{2 \pi}} \exp \left(-\frac{b^{2}}{2 \sigma^{2}}\right)
$$

where 


$$
\begin{aligned}
& \sigma^{2}=\sigma_{z}{ }^{2}=\int_{-\infty}^{\infty} \Phi_{z z}(\omega) d \omega \\
& \sigma_{1}{ }^{2}=\int_{-\infty}^{\infty}\left(\omega^{2}-\omega_{m}{ }^{2}\right) \Phi_{z z}(\omega) d \omega
\end{aligned}
$$

$\Phi_{Z Z}(\omega)$ is the spectral density and $\omega_{\mathrm{m}}$ is a representative mid-band frequency of the narrow-band random response process (i.e. natural frequency).

Apart from the time to first-passage, the probability distribution of the peaks of the random process is also of interest. A peak value in a sample function $z(t)$ of a continuously valued random process $Z(t)$, which is also continuous with respect to time, occurs when $\dot{z}(t)=0$. Therefore, analysis of the peak distribution is a zero-crossing problem of the first derivative $\dot{z}(t)$. For a stationary zero mean Gaussian process, the probability density of the peaks is given by:

$\left.p_{P}(a)=\frac{\sqrt{1-\alpha^{2}}}{\sigma_{Z} \sqrt{2 \pi}} \exp \left\{-\frac{b^{2}}{2 \sigma_{Z}^{2}(1-\alpha)^{2}}\right]\right\}+\frac{b \alpha}{2{\sigma_{Z}}^{2}}\left\{1+\operatorname{erf}\left[\frac{b}{\sigma_{Z} \sqrt{\frac{2}{\alpha^{2}}-2}}\right]\right\} \exp \left(-\frac{b^{2}}{2 \sigma_{Z}^{2}}\right)$

For a narrow-band random process, $\alpha=1$ and the probability density for the peak magnitude reduces to:

$$
p_{P}(b)=\frac{b}{{\sigma_{Z}}^{2}} \exp \left(-\frac{b^{2}}{2 \sigma_{Z}{ }^{2}}\right)
$$

Equation [34] represents the Rayleigh distribution, which is a special case of the Extreme Value Type III distribution known as the Weibull distribution. This same result for a narrow-band process is obtained by considering that the proportion of cycles for which $Z$ 
$>b$ is simply $v^{+} / v_{0}^{+}$, where $v^{+}$is the upcrossing rate given by Eq. [29] and $v_{0}^{+}$is the rate at which cycles occur given by Eq. [30]. The ratio is equivalent to $1-F_{p}(b)$ and therefore by differentiation $p_{\mathrm{F}}(b)$ in Eq. [34] is obtained.

\subsection{Markov Process Theory}

Under the assumption of a Markov response process a number of researchers have proposed approximate solutions to the first-passage problem. Among these, for a discrete random process, the random walk model (Toland and Yang 1971) is well known. For the continuous case, first-passage problem solutions are based on the FPK equation governing the evolution of the transition probability density function in the phase plane. For first-passage problems, the transition probability density function, which describes the instantaneous joint distribution of $z$ and $\dot{z}$ over the phase plane, is called the probability mass. This term is derived from visualizing the joint distribution as a distribution of mass over the phase plane. During the evolution of a random process beginning at $t=0$, the probability mass spreads out from the initial starting distribution and its centre advances along a clockwise spiral trajectory in the phase plane. The rate at which probability mass crosses an absorbing boundary (type-B, type-D or type-E) and is lost defines the first-passage probability density.

There are two approaches for determining the rate of loss of probability mass, one is based on the Numerical Diffusion Technique (Sec. 2.2.1.1.2.5) and the other uses the Finite Element Method (Sec. 2.2.1.1.2.2). The application of Numerical Diffusion to the first-passage problem was pioneered by Crandall et al. (1966) and later extended by Sun and Hsu (1988) using their Generalized Cell Mapping Algorithm. The Finite Element solution of the first-passage problem was initially developed using a Petrov-Galerkin method to solve the backward Kolmogorov equation, which is the formal adjoint of the forward Kolmogorov equation or FPK equation (Bergman and Spencer 1983, Spencer 1986). A solution for the first-passage problem was later developed directly from the solution of the FPK equation using a Bubnov-Galerkin method (Spencer and Bergman 1991). 
Results from the Numerical Diffusion and Finite Element studies of the firstpassage problem indicate that the general form of the first-crossing density given by Eq. [22], which is based on the assumption of independent events, is valid for both linear and non-linear systems.

\subsubsection{Perturbation Method}

This method may be applied to any continuous or discrete multiple-degree-offreedom system having small non-linearities such that the governing equations of the system may be expressed in a solvable linear form. Specifically, the solution to a nonlinear set of equations is expanded in terms of a small scaling parameter $\varepsilon$ that characterizes the magnitude of the non-linear terms involved. Perturbation theory has been used for deterministic vibration analysis for many years and was generalized to the case of stochastic excitation, in particular non-linear systems, by Crandall (1963).

In the case of a single-degree-of-freedom system having small non-linearities $\eta$, the equation of motion may be written as:

$$
\ddot{z}+\dot{\gamma}+\omega_{0} z+\varepsilon \eta(z, \dot{z})=F(t)
$$

where $\gamma, \omega_{0}$, and $\varepsilon$ are constants and $\varepsilon<<1$. If the perturbations are of the order $\varepsilon$ or smaller then the response may be written as a power series in $\varepsilon$ as follows:

$$
z(t)=z_{0}(t)+\varepsilon z_{1}(t)+\varepsilon^{2} z_{2}(t)+\cdots
$$

Taking derivatives of the above series expansion, the resulting expressions for $z, \dot{z}$ and $\dot{z}$ may be substituted into the equation of motion of the non-linear system, Eq. [35]. Equating terms of the same order in $\varepsilon$ results in a set of linear equations of motion for $z_{0}$, $z_{l}, z_{2} \ldots$. One could write as many equations as desired for higher orders of $\varepsilon$. Clearly, 
the accuracy of the power series expansion of the response depends on the number of terms retained in the series. However, the mathematical problems and uncertain convergence criteria associated with finding solutions including higher than first-order terms are usually too complicated to be of practical use. Therefore, typically, the Perturbation Method is limited to first-order perturbation in which only terms of the order $\varepsilon$ are included.

The solution for the displacement responses $z_{0}(t), z_{1}(t), z_{2}(t) \ldots$, which are summed to yield the complete solution, are obtained with the use of the convolution integral, which gives the response of a linear dynamic system to a series of impulses. Infinitesimally short impulses are used to represent the arbitrarily varying force $F(t)$ since the system response to a unit impulse is known and superposition may be used on the linearized system to determine the response to a series of impulses. Taking an example from Branstetter et al. (1988), if $\eta(z, \dot{z})=z \dot{z}$ in Eq. [35], then the resulting linear equations for terms of order zero and one in $\varepsilon$ are:

$$
\begin{aligned}
& \ddot{z}_{0}+\gamma \dot{z}_{0}+\omega_{0} z_{0}=F(t) \\
& \ddot{z}_{1}+\gamma \dot{z}_{1}+\omega_{0} z_{1}=-z_{0} \dot{z}_{0}
\end{aligned}
$$

The solution for the first of Eqs. [37] is given by the convolution integral as follows:

$$
z_{0}(t)=\int_{-\infty}^{\infty} h(t-\tau) F(\tau) d \tau
$$

where $h()$ is the impulse response function of the linear system. For a single-degree-offreedom system, the convolution integral, of course, specializes to Duhamel's integral. Knowing $z_{0}(t)$ from Eq. [38], the second of Eqs. [37] is solved using the following convolution:

$$
z_{1}(t)=\int_{-\infty}^{\infty} h(t-\tau)\left(-z_{0}(\tau) \dot{z}_{0}(\tau)\right) d \tau
$$


In this way, as many terms as desired in the series expansion for $z(t)$ may be evaluated, however, as stated previously, typically only first-order order terms in $\varepsilon$ are considered.

If $z(t)$ is stochastic, then the mean function and the auto-correlation function for the response of the example system are determined from the expected value of each convolution as follows (Lin 1967):

$$
\begin{aligned}
& E[Z(t)]=E\left[Z_{0}(t)\right]+\varepsilon E\left[Z_{1}(t)\right] \\
& E[Z(t)]=\int_{-\infty}^{\infty} E[F(t)] h(t-\tau) d \tau-\varepsilon \int_{-\infty}^{\infty} E\left[z_{0}(\tau) \dot{z}_{0}(\tau)\right] h(t-\tau) d \tau \\
& E[Z(t+\tau) Z(t)]=E\left[Z_{0}(t+\tau) Z_{0}(t)\right]+\varepsilon E\left[Z_{0}(t+\tau) Z_{1}(t)\right]+\varepsilon E\left[Z_{0}(t) Z_{1}(t+\tau)\right] \\
& R_{Z Z}(\tau)=R_{Z_{0} Z_{0}}(\tau)+\varepsilon\left[R_{Z_{0} Z_{1}}(\tau)+R_{Z_{1} Z_{0}}(\tau)\right]
\end{aligned}
$$

Although the Perturbation Method is applicable to multiple-degree-of-freedom systems and is not confined to a white-noise assumption for the excitation, it is limited to weakly non-linear systems and as such is not applicable to ductile, hysteretic systems.

\subsubsection{Equivalent Linearization Method}

The method of Equivalent Linearization was originated in the 1930's for the treatment of non-linear systems under deterministic excitations. It was first extended to the case of random excitation independently by Botoon (1954) and Caughey (1960) and later generalized for multiple-degree-of-freedom systems by Iwan (1973). In this method, the stochastic equation governing a non-linear system is replaced by an 'equivalent' linearized version which introduces a random error between the true nonlinear and linearized systems. This error is minimized, usually in a mean-square sense, by setting to zero the partial derivatives of the expected value of the squared error with respect to the coefficients appearing in the linearized equation. These partial derivatives define a set of equations, which are then solved for the required coefficients. 
In the case of a single-degree-of-freedom stochastic system, the governing nonlinear differential equation is given by:

$$
\ddot{z}+h(z, \dot{z})=G(t)
$$

Assuming the function $h(z, \dot{z})$ containing non-linear terms related to the system damping and stiffness may be approximately written as the sum of two linear components, one pertaining to damping and the other to stiffness, the governing differential equation is linearized to:

$$
\ddot{z}+\alpha \dot{z}+\beta z=G(t)
$$

The error introduced by the linearization is then the difference between the non-linear $h$ function and the two linear components:

$$
\varepsilon=h(z, \dot{z})-\alpha \dot{z}-\beta z
$$

This error is a random process and must be minimized for the best prediction of system response. The usual means of minimizing the error is to minimize the mean-square error, which is accomplished by requiring that:

$$
\begin{aligned}
& \frac{\partial}{\partial \alpha} E\left[\varepsilon^{2}\right]=0 \\
& \frac{\partial}{\partial \beta} E\left[\varepsilon^{2}\right]=0
\end{aligned}
$$

Substituting the error equation, Eq. [43], into the partial derivatives of Eqs. [44], the coefficients $\alpha$ and $\beta$ which minimize $\varepsilon$ are given by (Lin 1967): 


$$
\begin{aligned}
& \alpha=\frac{E\left[Z^{2}\right] E[\dot{Z} \cdot h(Z, \dot{Z})]-E[Z \dot{Z}] E[Z \cdot h(Z, \dot{Z})]}{E\left[Z^{2}\right] E\left[\dot{Z}^{2}\right]-\left(E[Z \dot{Z})^{2}\right.} \\
& \beta=\frac{E\left[\dot{Z}^{2}\right] E[Z \cdot h(Z, \dot{Z})]-E[Z \dot{Z}] E[\dot{Z} \cdot h(Z, \dot{Z})]}{E\left[Z^{2}\right] E\left[\dot{Z}^{2}\right]-(E[Z \dot{Z}])^{2}}
\end{aligned}
$$

Note that Eqs. [45] are not explicit expressions for $\alpha$ and $\beta$ since the expectations appearing on the right-hand sides depend on $\alpha$ and $\beta$. Evaluation of $\alpha$ and $\beta$ is simplified if the excitation $G(t)$ is assumed to be stationary and Gaussian with a zero expectation and the non-linearities in stiffness and damping are separable, i.e.:

$$
h(z, \dot{z})=f_{1}(z)+f_{2}(\dot{z})
$$

However, these conditions are not essential for determining $\alpha$ and $\beta$. In general, the linearized system coefficients are functions of the unknown, usually non-Gaussian, response statistics and other statistics involving the restoring force. For this reason an iterative solution procedure is generally required to determine $\alpha$ and $\beta$.

The first application of Equivalent Linearization techniques to hysteretic systems subjected to random excitation was given by Caughey (1960) who modelled a bilinear system using the Krylov-Bogoliubov (K-B) assumption of slowly varying parameters. However, similar to the results of Iwan and Lutes (1968), the K-B assumption may lead to serious underestimates in the RMS response of the system (see Sec. 2.2.1.1.2.4). To eliminate reliance on the K-B assumption, Wen (1980) and Baber and Wen (1981) incorporated Bouc's (1967) smooth hysteresis model, which models a hysteretic, deteriorating system with non-linear differential equations, to obtain a solution for the system linearization coefficients. Their solution for the response statistics of both stationary and non-stationary hysteretic degrading systems utilized a convenient procedure suggested by Atalik and Utku (1976) to estimate the linearization coefficients in multiple-degree-of-freedom systems. The modelling and solution procedure developed by Wen was later extended to systems under non-zero mean excitation (Baber 
1984) and also to systems exhibiting pinching of the hysteresis loops (Baber and Noori 1985). With these improvements, the Equivalent Linearization Method utilizing the smooth hysteresis model has been successfully applied to response and damage prediction of a variety of structural systems under seismic excitation.

The accuracy of the Equivalent Linearization Method, as measured against exact FPK solutions of certain systems and Monte Carlo simulation, is generally very good with an error of less than 20 percent considered representative (Branstetter et al. 1988). Furthermore, unlike most approximate analytical methods, the accuracy of Equivalent Linearization is relatively independent of the severity of the non-linearity, be it of geometric or material source. The error in mean-square response remains quite small even for large non-linearities (Roberts 1981). Caution is necessary, however, when the excitation spectral content is such that the power spectral density function vanishes rapidly as the frequency goes to zero. In this case, which is typical of earthquake excitation, the method tends to underestimate the displacement response. The error depends largely on the characteristics of the system restoring force and the excitation in the low frequency range. It is negligible when the power spectral density function is non-zero at zero frequency but for an earthquake excitation model with a power spectral density which goes to zero at zero frequency, the RMS response could be underestimated by $20-30 \%$ (Wen 1989).

In addition to the caution necessary when using Equivalent Linearization for seismic response analysis, it should be noted that the assumption of a Gaussian input results in an assumed Gaussian response of the linearized non-linear system. This assumption is not correct for a non-linear system, which is known to have a nonGaussian response to a Gaussian input. The result of an assumed Gaussian response is that it may significantly misrepresent the frequency of high response levels to extreme loads, which contribute most to first-passage and fatigue failures. Instead of assuming a Gaussian distribution for the response variables, the accuracy of the method may be improved by including higher order non-Gaussian effects. Full probability distributions have been estimated from non-linear response moments with Gram-Charlier and 
Edgeworth series (Crandall 1980), however, these series can behave erratically yielding negative probability densities and crossing rates for significantly non-linear systems. To alleviate this problem, Winterstein (1988) proposed a Hermite moment model in which response moments (skewness, kurtosis etc.) are used to form non-Gaussian response contributions made orthogonal through a Hermite series to minimize mean-square error. The Hermite moment model predicts full probability distributions of the response and its extremes as well as crossing rates and fatigue damage rate. If used with observed moments from a response time history, the model corrects for non-linearity without the need to fully specify or analyze a precise non-linear model. In analytical studies, the Hermite moment model can be combined with various moment estimation techniques such as Moment Closure (see Sec. 2.2.1.1.2.3).

\subsubsection{Functional Series Representation}

The Functional Series Representation Method is similar to the Perturbation Method in that it is limited to systems in which the non-linearities are small. Given that the response of a linear system may be expressed in the form of a convolution as given in Eq. [38], it has been shown (Wiener 1958) that the convolution may be generalized to a Volterra series expression when the system is non-linear:

$$
z(t)=\sum_{n=1}^{\infty} \int_{-\infty}^{\infty} \ldots \int_{-\infty}^{\infty} h_{n}\left(t_{1}, t_{2}, \ldots, t_{n}\right) \cdot F\left(t-t_{1}\right) \ldots F\left(t-t_{n}\right) d t_{1} \ldots d t_{n}
$$

The function $h_{n}$ may be regarded as the $n$th degree impulse response function. Bedrosian and Rice (1971) later obtained the $n$th degree frequency response function $H_{n}$ in terms of system parameters using a system with harmonic input of the form:

$$
F(t)=\sum_{k=1}^{\infty} e^{i t \omega_{k}}
$$


Using this input and substituting into the Volterra series expression and then into the equation of motion, the $n$th degree frequency response function $H_{n}$ is obtained. Once $H_{n}$ is determined, $h_{n}$ in Eq. [47] is evaluated using the Fourier transform pair relationship given by the following:

$$
\begin{aligned}
& h_{n}\left(t_{1}, \ldots, t_{n}\right)=\frac{1}{(2 \pi)^{n}} \int_{-\infty}^{\infty} \cdots \int_{-\infty}^{\infty} H_{n}\left(\omega_{1}, \ldots, \omega_{n}\right) \cdot e^{i\left[\omega_{1} t_{1}+\cdots+\omega_{n} t_{n}\right]} d \omega_{1} \ldots d \omega_{n} \\
& H_{n}\left(\omega_{1}, \ldots, \omega_{n}\right)=\int_{-\infty}^{\infty} \ldots \int_{-\infty}^{\infty} h_{n}\left(t_{1}, \ldots, t_{n}\right) \cdot e^{i\left[\omega_{1} t_{1}+\cdots+\omega_{n} t_{n}\right]} d t_{1} \ldots d t_{n}
\end{aligned}
$$

Once $h_{n}$ is evaluated the response of the Volterra series expression, and therefore the non-linear system response, is completely determined. This method may also be used to evaluate the response statistics of weakly non-linear systems.

\subsubsection{Decomposition Method}

This method is an operator-based technique originally proposed by Adomian (1983) and later extended by Benaroya (1984) to analyze non-linear problems in structural dynamics. This method, which has received limited attention in the literature, does not require any of the assumptions concerning the characteristics of the forcing function and/or system non-linearity which are used in the Fokker-Planck, Equivalent Linearization, Perturbation and Functional Series Methods. It is particularly suited to systems with random properties, for example, systems in which stiffness is a random function of time. As summarized by Branstetter et al. (1988), consider the following linear system where the stiffness $k(t)$ is a function of time only, and is therefore not dependent on the load:

$$
m \ddot{z}(t)+c \dot{z}(t)+k(t) z(t)=F(t)
$$

where $m$ and $c$ are constants and $k(t)$ is a random process. Define the linear operator $L$ as: 


$$
L=m \frac{d^{2}}{d t^{2}}+c \frac{d}{d t}+k(t)
$$

Then the equation of motion in operator form is:

$$
L[z(t)]=F(t)
$$

The system stiffness may be written as the sum of deterministic and random parts where the deterministic part is the mean value of $k(t)$, given by $E[k(t)]$, and the random part, denoted by $K(t)$, is the random fluctuation about the mean:

$$
k(t)=E[k(t)]+K(t)
$$

Similarly, the operator $L$ may be separated into a deterministic part $D$ and random part $R$ resulting in $L=D+R$, where:

$$
\begin{aligned}
& D=m \frac{d^{2}}{d t^{2}}+c \frac{d}{d t}+E[k(t)] \\
& R=K(t)
\end{aligned}
$$

Substituting $L=D+R$ into the operator form of the equation of motion results in:

$$
z=D^{-1} F-D^{-1} R z
$$

Now, let $z$ be written as the sum of a series:

$$
z(t)=\sum_{i=0}^{\infty} z_{i}(t)
$$

Substituting Eq. [56] into Eq. [55], the equation of motion becomes: 


$$
z=D^{-1} F-D^{-1} R\left(z_{0}+z_{1}+\ldots\right)
$$

The series expression for $z(t)$, Eq. [56], may be regrouped as follows:

$$
\begin{aligned}
& z_{0}(t)=D^{-1} F \\
& z_{1}(t)=-D^{-1} R z_{0}=-D^{-1} R D^{-1} F \\
& z_{2}(t)=-D^{-1} R z_{1}=D^{-1} R D^{-1} R D^{-1} F
\end{aligned}
$$

Therefore, Eq. [56] may be re-written as:

$$
z(t)=\sum_{i=0}^{\infty}(-1)^{i}\left(D^{-1} R\right)^{i} D^{-1} F
$$

In practice, of course, this series expression for the solution of the non-linear system will be truncated to a finite number of terms, which introduces some error. Finally, the deterministic operator $D$ has to be inverted to allow computation of the response statistics. If the inverse $D^{-1}$ exists and has a corresponding Green's function $g(t, \tau)$, sometimes called the weighting function, then Eq. [59] may be written in integral form as:

$$
z(t)=\int_{0}^{t} g(t, \tau) F(\tau) d \tau-\int_{0}^{t} g(t, \tau) K(\tau) z(\tau) d \tau+A_{1} z_{1}(t)+A_{2} z_{2}(t)
$$

where $z_{1}(t)$ and $z_{2}(t)$ solve the homogeneous equation $L[z]=0$ and $A_{1}$ and $A_{2}$ are determined by initial conditions. Statistics of the response may be computed using Eq. [60], which was derived without using any assumptions on the nature of the forcing function. 


\subsubsection{Numerical Methods}

In contrast to the analytical random vibration methods described in Section 2.2.1, which apply in the frequency domain, numerical methods are time domain based. The time-history approach to finding the response statistics of a dynamic system is generally more accurate and robust than any of the random vibration methods described previously. The inherent assumptions in each of the analytical procedures, for instance the whitenoise assumption of the Markov based methods or the assumption of weak non-linearity in the Perturbation and Decomposition Methods, are not required to obtain response statistics using time domain based numerical methods. In the context of civil engineering structures, dynamic analysis of a structure subjected to a simulated load time-history, using a numerical integration scheme, will yield a response time-history. An ensemble of these can be used to estimate the response statistics of the system at any time in the response process without regard to the nature of the restoring force (i.e. degree of nonlinearity), complexity of the structural system (i.e. number of degrees of freedom) or nature of the random excitation process. This generality is the reason that numerical methods are frequently used to verify results obtained using other analytical random vibration methods.

The greatest drawback to the numerical approach is the computational cost. One time-history calculation corresponds to a single sample of response, whereas the more efficient random vibration methods yield, at least in principle, the complete statistical response of the system in their solution. Also, for structural reliability problems, computation of the response statistics is only the first step in a two-step process. The second step, which makes use of the response statistics, is the approximation of the probability of failure using any of a variety or combination of methods: distribution fitting of response statistics, a response surface approach, FORM/SORM, Direct Monte Carlo simulation, and Selective Monte Carlo simulation using variance reduction techniques. The computational cost of generating time-history response statistics is dependent on the reliability analysis method used to solve for the probability of failure. With this in mind, the origin of the numerical approach to solving complex stochastic 
non-linear dynamic systems is summarized below followed by a brief description of the techniques that were subsequently developed to reduce computation time.

\subsubsection{Direct Monte Carlo Simulation}

The principle of Monte Carlo simulation seems to have originated with Buffon's Needle Experiment in the $18^{\text {th }}$ century. Buffon's original experiment was to drop a needle of length $L$ at random on a grid of parallel lines of spacing $D$ where $D \geq \mathrm{L}$ from which the value of $\pi$ could be inferred by observing the number of intersections between needle and lines. The approximation of $\pi$ is given by:

$$
\pi \cong \frac{2 \cdot L \cdot N}{R \cdot D}
$$

where $N$ is the number of needle drops and $R$ is the number of intersections.

Buffon's Needle Experiment and a few other applications of random sampling pre-date the naming and systematic development of the Monte Carlo Method, which began in about 1944. The name is taken from Monte Carlo, Monaco where the Roulette wheel, which is a simple random number generator, is synonymous with the city. The development of Monte Carlo simulation as a research tool stems from work on the atomic bomb during the Second World War by mathematicians John von Neumann and Stanislaw Ulam. This work involved a direct simulation of the probabilistic problems concerned with random neutron diffusion in fissile material. Since that time Monte Carlo simulation has been applied to a wide variety of mathematical problems involving stochastic and dynamic systems in fields ranging from economics to the natural sciences to engineering.

Although Monte Carlo methods quite often offer the only available solution to a complex stochastic system, Direct Monte Carlo simulation is not well adapted to solving dynamic reliability problems. The reason for this is that the probability of failure being 
estimated governs the number of sample responses or realizations that are required. Assuming a binomial distribution for the quantity being estimated, the relative error in a Monte Carlo estimate is given by (Melchers 1999):

$$
\varepsilon=k \sqrt{\frac{1-p}{n p}}
$$

where $k$ is the standard normal variable associated with a given confidence interval, $n$ is the number of realizations and $p$ is the expected probability of occurrence. Note that Eq. [62] does not depend on the dimension of the state space (i.e. the number of degrees of freedom in the structural system). Since, typically, dynamic systems under service must be very reliable, failures or malfunctions are rare events and therefore, from Eq. [62], the number of realizations required for an accurate estimate of a small probability of failure is very large. Assuming that the observed value of the probability of failure of a system is required to be within $5 \%$ of the true value with $95 \%$ confidence, then for a system with an expected probability of failure of $10^{-6}, 1.54 \times 10^{9}$ realizations would be required. Clearly, without the use of supercomputers and parallel processing (Johnson et al. 1997) it is impractical to generate this many samples for realistic problems where each response computation may require dynamic analysis of a non-linear structural system.

\subsubsection{Selective Monte Carlo Simulation}

The slow convergence of the Direct Monte Carlo estimate, where the error decreases in proportion to $n^{-1 / 2}$, has led directly to the so-called 'variance reduction' techniques used in Selective Monte Carlo simulation to reduce the computational cost of estimating low probability events. A number of techniques such as: Antithetic Variates (Ayyab and Haldar 1984), Conditional Expectation (Ayyub and Chia 1991) and Importance Sampling (Kahn 1956) were developed for static reliability problems but are not well suited to dynamic systems. This may be illustrated by considering the case of Importance Sampling in which an importance sampling probability density function $h(x)$ is located on the limit state surface at the point of maximum likelihood (or the design 
point) of the joint probability density function $f(x)$ of the random variables in standard normal space. Parenthetically, the design point is usually not known prior to the reliability analysis and must be located by application of numerical maximization techniques, a search algorithm or more recently by application of a neural network. Once positioned, the sampling density is used to increase the number of random samples that fall into the failure domain in the standard normal space thereby reducing the variance of the Monte Carlo estimate of the failure probability for a given number of trials. Note that to offset the expected distortion of the original joint density function by the sampling distribution, the weight of each sample must be modified by $1 / h(x)$. The difficulty in applying this technique to a dynamic system is two-fold. Firstly, unlike the static case, the joint density function of the random variables is not known, instead there is only a finite sample of realizations. Second, the application of Importance Sampling to a dynamic system would require a description of the sampling density function with respect to time, which greatly increases the problem complexity.

To handle dynamic reliability problems, two variance reduction techniques have been developed that model the flow of probability into the low probability region of the phase plane.

\subsection{Double and Clump}

Since the flow of probability into the low probability region is of paramount interest in reliability analysis, Pradlwarter et al. (1994) developed a procedure they named 'Double and Clump' (D\&C) which provides a means to increase the sample size of important realizations falling in the failure domain. The basic idea is that in the event that a sample realization is identified as important at a given time step, based on specific energy and weight criteria, the state vector in the phase plane is doubled. Doubling of a realization at a certain time step $\tau$ simply means that an identical copy of the state vector is made and its weight is halved. The effect of doubling can only be observed at a time $t$ $>\tau$ since starting at $t=\tau$, the loading increments are assumed to be independent in both doubled state vectors. Hence, two different random paths in the state space will be 
observed due to the differences in loading for $t>\tau$. In order to preserve the total number of realizations, those trajectories that do not meet the energy and weight criteria for doubling are clumped together and the weight of each realization is combined. The varying magnitude of the weights of all realizations, which change discontinuously in time, represents a generalization of Direct Monte Carlo simulation in which all weights are equal $(=1 / n)$ and constant in time. In Importance Sampling, weight can be interpreted as the ratio between the joint density and the sampling density $(f(x) / h(x))$, which changes with position in the standard normal space but not as a function of time.

To ensure accuracy of the Double and Clump procedure it is important that the statistics of the samples are not significantly affected. Doubling has no effect on the sample statistics but clumping does since it is impossible to maintain the same amount of information with fewer samples. Clumping to the mean value of two state vectors ensures identical first moments before and after clumping, however, higher moments are generally slightly underestimated after clumping. The distortion of higher moments depends mainly on the distance in the phase plane between the realizations to be clumped. Therefore, in order to minimize distortion, only realizations close to each other are permitted to clump. This proximity requirement, however, increases the computational cost of $\mathrm{D} \& \mathrm{C}$ since all realizations, which are simulated simultaneously, must be searched repeatedly during the time stepping procedure for a sufficiently close partner to clump with.

\subsection{Russian Roulette and Splitting}

To alleviate the problem of searching all realizations for a clumping partner in D\&C, Pradlwarter and Schueller (1997) proposed a similar Selective Monte Carlo method called 'Russian Roulette and Splitting' (RR\&S) which was an adaptation for stochastic dynamic systems of a method first developed and applied for estimating low probability events in neutron shielding problems. In RR\&S, instead of searching for two close realizations suitable for clumping, an 'unimportant' realization is simply killed off and the weights of all other realizations are normalized with respect to the probability of 
survival of each realization before the killing of the unimportant sample. To ensure preferential survival of important realizations, which are those that enter an important region in the phase plane, a higher survival probability is associated with them. The name Russian Roulette is derived from this probabilistic method of determining which realizations are killed, while Splitting was used to describe the doubling of important particles in the original neutron shielding problems.

In a general complex stochastic dynamic system it may be difficult to determine a measure to distinguish between important and unimportant regions. For this reason, Pradlwarter and Schueller (1999) proposed 'Distance Controlled' Monte Carlo simulation, which combines $R R \& S$ with an evolutionary technique to determine the realization selection criterion. This evolutionary technique is somewhat different from the use of Genetic Algorithms, which was proposed by Johnson et al. (1996). The proposed method is shown to predict extremely low probability events as well as the ability to analyze complex dynamic systems for which other methods do not appear to be suitable.

\subsection{Latin Hypercube Sampling}

Latin Hypercube Sampling is a technique that provides a constrained sampling scheme, instead of the purely random sampling of Direct Monte Carlo simulation, to reduce the variance of a Monte Carlo estimator for a given number of random samples (Imam and Conover 1980, Ayyub and Lai 1989). For this reason, Latin Hypercube Sampling is called a selective sampling scheme.

In traditional random sampling, random numbers between 0 and 1 are generated and these are then used to generate random variables according to the prescribed distribution function for each variable. Typically, the inverse transformation method is used with the cumulative distribution function (CDF) to map the random numbers to the generated random variables. Other procedures for generating random variates include the Composition Method and the Acceptance-Rejection Method attributed to von Neumann 
(Rubinstein 1981). In Latin Hypercube Sampling, the density function or CDF of each variable is divided into $n$ non-overlapping intervals of equal probability, where $n$ is the number of random values that have been chosen for Monte Carlo simulation. A representative value from each interval is then chosen for each variable and randomly matched without replacement to a value for each of the other random variables. In this way, the entire range of each random variable is represented in the set of variables to be used in a Monte Carlo simulation. For example, if 10 Monte Carlo simulations were used to estimate the mean value of a performance function that contained 5 random variables, then the density function of each random variable would be divided into 10 intervals and 10 sets of 5 values would be generated. Each set is obtained by randomly matching one interval value from each of the 5 variables together.

A proposed variant of Latin Hypercube Sampling called Updated Latin Hypercube Sampling, which further reduces the variance in Monte Carlo estimates of commonly used statistical parameters such as mean, standard deviation, coefficient of variation and CDF, was developed by Florian (1992). This was followed by the work of Huntington and Lyrintzis (1998) who proposed two techniques to even further improve the performance of Latin Hypercube Sampling, at the cost of significantly longer computation time.

\subsubsection{Response Surface Method}

Response surface methodology (RSM) is a collection of statistical analysis methods that examines the relationship between experimental response and variations in the values of the input variables. Developed by research scientists performing experiments in biology and agriculture, it is intended to create and analyze statistical models of processes that are difficult to study directly for reasons of complexity, or because the underlying mechanism controlling the process is not well understood or the response data is expensive to produce (Myers 1976). In the context of structural reliability analysis, the functional relationship between the structural response and the input variables is approximated by a response surface model, which is then used in a 
conventional reliability analysis method such as FORM/SORM or Monte Carlo simulation.

Using RSM to study the influence of variables on an outcome or process consists of two phases, response surface design followed by the analysis phase. Response surface design is the process of deciding on an experimental strategy that determines the number of variables and what combination of variable levels should be used in an experiment to generate an outcome. Each outcome, which is then used to fit the surface, involves a certain cost or computation time, therefore, the response surface design should be as efficient as possible at fitting the surface in the area of interest. Since the actual form and degree of the surface are not known ahead of time, only discrete experimental outcomes are known, there is little guidance in the selection of the approximating surface, however, a second-order polynomial is typically used. The general form of a second order approximating function is given by:

$$
E(y)=\beta_{0}+\sum_{i=1}^{k} \beta_{i} x_{i}+\sum_{i=1}^{k} \beta_{i i} x_{i}^{2}+\sum_{i=1}^{k} \sum_{j=1}^{i-1} \beta_{i j} x_{i} x_{j}
$$

where $E(y)$ is the expected value of the response, $k$ is the number of independent variables, $\beta_{i}$ and $\beta_{i j}$ are the regression coefficients and $x_{i}$ is the $i$ th variable. Box and Wilson (1951) introduced an efficient class of designs for fitting second-order surfaces called central composite designs, which consists of a $2^{k}$ factorial design, with each variable at the two normalized levels of -1 and +1 , augmented by $2 k$ axial points and $n_{2}$ center points for a total of $2^{k}+2 k+n_{2}=N$ points. This design results in a significantly reduced number of experimental points from a $3^{k}$ design, with each variable at three levels, which would normally be required to fit a second-order surface.

Once the $N$ design points have been chosen, the regression coefficients $\beta_{i}$ and $\beta_{i j}$ are determined using the method of least squares, which minimizes the total error between the predicted response from Eq. [63] and the actual experimental outcome. The total error resulting from the use of the fitted response surface is comprised of two 
elements, pure error and lack-of-fit error. Pure error comes from the intrinsic randomness of the system and cannot be eliminated, while lack-of-fit error is the result of the inability of the response surface model to represent the true response with a simple polynomial expression. For the response surface model to be considered an accurate representation of the true response, it is required that the lack-of-fit error can be neglected. The means of measuring lack-of-fit error, so that a decision criterion for accepting a response surface model may be established, is provided by the analysis of variance technique (ANOVA). Using ANOVA, Faravelli (1989) suggested an expression involving the pure error and lack-of-fit error for evaluating the goodness-offit of a response surface. Böhm and Brückner-Foit (1992) proposed the use of two alternative criteria based on the lack-of-fit error to validate a response surface, stating that the measure suggested by Faravelli had no theoretical justification. The increased computational effort required by the Böhm and Brückner-Foit criteria led Yao and Wen (1996) to propose an empirical measure based on Faravelli's expression that reduced computation time.

The analysis phase of RSM involves the use of techniques such as: canonical analysis, method of steepest ascent and method of ridge analysis to analyze the experimental information in the second order response model and draw conclusions regarding the influence of variables and the predicted outcome as the input variables change. 


\subsection{EARTHQUAKE GROUND MOTION MODELS}

The use of any of the numerical methods of Section 2.2.2 requires sample functions of the stochastic process, field or wave that is to be used as input to the system being analyzed. The generated sample functions. must accurately describe the probabilistic characteristics of the corresponding stochastic process, field or wave which may be either stationary or non-stationary, homogeneous or non-homogeneous, one dimensional or multi-dimensional, uni-variate or multi-variate, and Gaussian or nonGaussian. For purposes of definition, a stochastic process is an infinite population or ensemble whose samples are functions of time only, together with information concerning relative probabilities of sample values. A stochastic field is similar to a stochastic process except that the samples are functions of space rather than time, whereas a stochastic wave model incorporates probabilistic information regarding both time and space to generate sample functions. In the case where there is uniformity in the random process, field or wave, it is described by the designations stationary in time and homogeneous in space. A stationary process is one whose probability distributions across the ensemble are invariant with respect to translations in the origin of time. Similarly, a random field is homogeneous with respect to a particular spatial coordinate if its probability distributions are invariant with respect to translations of the origin along the axis of that coordinate.

A stationary or homogeneous random process or field can be described in terms of its spectral density, which is related to its auto-correlation function through the wellknown Wiener-Khintchine theorem (see Sec. 2.2.1.1.1). Spectral decomposition of a random process is extremely useful because the auto-correlation and spectral density functions provide average amplitude and frequency information about sample processes and, in the case of a linear time-invariant system, they provide the corresponding statistics of the stochastic dynamic response. Non-stationary random processes and fields, however, are more difficult to model since the concept of spectral density does not apply due to the fact that the auto-correlation function is no longer a function of the time-shift $\left(\tau=t_{1}-t_{2}\right)$ only, but depends on two independent time arguments $t_{1}$ and $t_{2}$. 
Several techniques have been developed to approximate a non-stationary process including: (1) the use of a generalized spectral density, defined by a double Fourier transform (Lin 1967), (2) the use of an evolutionary power spectrum (Priestley 1965, 1967), which may be used to describe relatively slow changes in frequency content of a non-stationary process, (3) modeling the non-stationary random process as a nonstationary shot-noise (Lin 1967), or (4) modeling the non-stationary random process as an 'equivalent' stationary random process modulated by a deterministic amplitude variation (Bolotin 1960).

In the fields of earthquake engineering and seismology, a large number of stochastic models for generating artificial ground acceleration records have been proposed. These models may be roughly divided into five categories, which are listed in order of earliest to most recent: (1) filtered white-noise and filtered Poisson process models, (2) spectral representation method, (3) stochastic wave theory, (4) autoregressive and moving average (ARMA) models, and (5) wavelet models. Selected references from a review of the first three catergories by Shinozuka and Deodatis (1988) and a review of ARMA models by Kozin (1988) are given in the following summary of each category.

\subsubsection{Filtered White-Noise and Filtered Poisson Process Models}

The first ground motion models (Housner 1947, Bycroft 1960) were stationary white-noise processes, which have a constant Fourier amplitude spectrum (or power spectral density). Later, it was recognized from analyses of strong-motion records that the energy content of ground motion is not uniformly distributed over all frequencies, but is concentrated in certain frequency regions. To incorporate this, Tajimi (1960), using the work of Kanai (1957), proposed the filtered white-noise model, which accounted for local site properties and a dominant frequency in the ground motion. The Kanai-Tajimi filter model, which has been used extensively in the past to describe strong ground motion, was later modified by Clough and Penzien (1975) to remove the inconsistency of unbounded ground velocity and displacement at zero frequency. This modified version 
of the non-white Kanai-Tajimi model is given by the following equation for the power spectral density of the ground acceleration (see Fig. 2.3):

$$
S_{a}(\omega)=S_{0} \cdot\left[\frac{1+4 \xi_{G}{ }^{2}\left(\frac{\omega}{\omega_{G}}\right)^{2}}{\left(1-\left(\frac{\omega}{\omega_{G}}\right)^{2}\right)^{2}+4 \xi_{G}{ }^{2}\left(\frac{\omega}{\omega_{G}}\right)^{2}}\right] \cdot\left[\frac{\left(\frac{\omega}{\omega_{H}}\right)^{4}}{\left(1-\left(\frac{\omega}{\omega_{H}}\right)^{2}\right)^{2}+4 \xi_{H}{ }^{2}\left(\frac{\omega}{\omega_{H}}\right)^{2}}\right]
$$

where the first bracket represents the low-pass Kanai-Tajimi filter and the second bracket represents the high-pass Clough-Penzien filter. $S_{0}$ is the constant white-noise spectrum scaled to the energy of the ground motion, $\xi_{G}$ is the predominant frequency of the ground motion, which is indicative of the geological character of the local subsoil, $\omega_{\mathrm{G}}$ is the equivalent damping based on the hardness of the subsoil, $\xi_{H}$ and $\omega_{H}$ are empirical parameters that are determined by matching actual ground motion recordings from the site to ensure the correct frequency content of the artificial earthquake. More recently, seismologists have developed a wide variety of theoretical Fourier amplitude spectrum models that are based on the physical parameters of the earthquake source and medium such as: magnitude, distance, fault dimension, attenuation parameters, shear-wave velocity, wave propagation velocity etc. (Sólnes 1997). These models are highly specialized and, as such, outside the scope of this study and therefore, will not be reviewed further.

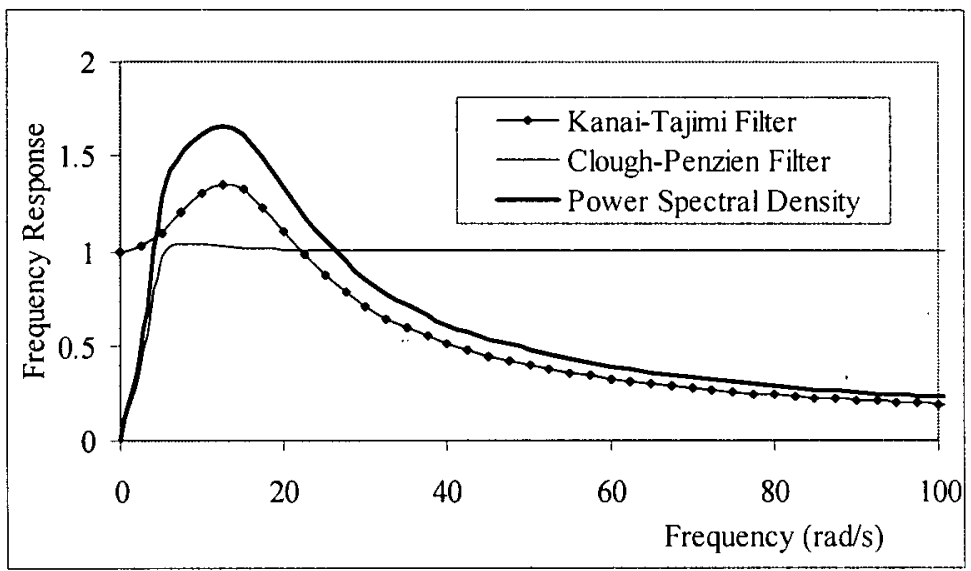

Figure 2.3: Filtered White-Noise Power Spectrum 
Following the work of Kanai and Tajimi, a variety of time-modulating functions were introduced to produce non-stationary ground motion models that reflected the time varying intensity (or amplitude non-stationarity) typical of real earthquake ground motion accelerograms. Since an earthquake motion is esssentially an evolutionary process, the filtered output sample functions have to be amplitude modulated to resemble the time evolution of the real motion, that is, show a build-up phase, a strong motion phase and an attenuating tail (see Fig. 2.4). The proposed time-modulating models include: time-modulated harmonics (Bogdanoff et al. 1961), filtered modulated whitenoise (Bolotin 1960, Housner and Jennings 1964, Amin and Ang 1968, Iyengar and Iyengar 1969, Ruiz and Penzien 1971) and the filtered modulated Poisson process (Cornell 1960, Shinozuka and Sato 1967, Lin 1963, 1965). In each model there are typically a number of constants that completely define the time-modulating envelope function, including the total duration of the earthquake. These constants depend on the magnitude of the earthquake, the distance from the causative fault and the focal depth.

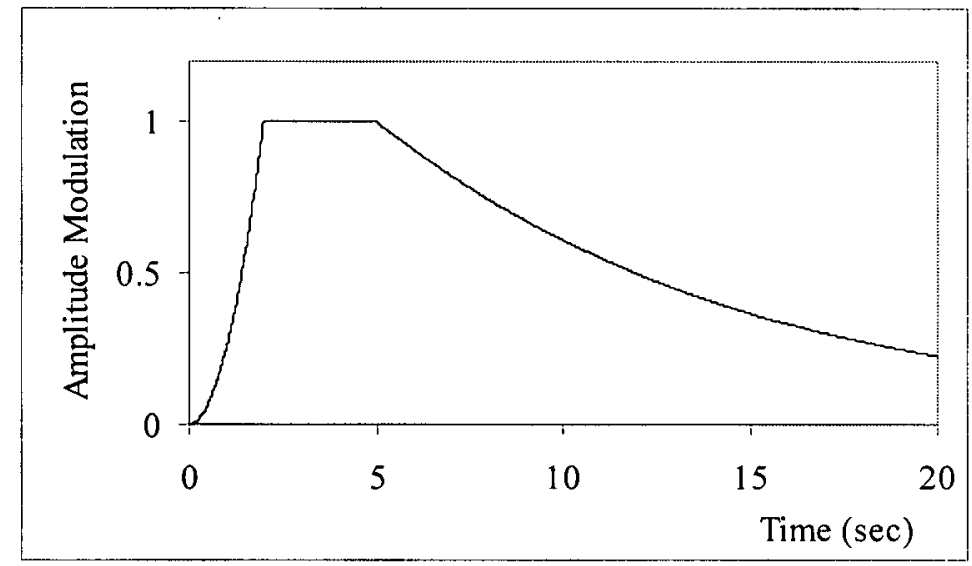

Figure 2.4: Example Amplitude Modulating Function (Amin and Ang 1968)

From a physical interpretation standpoint, the filtered Poisson process model is closer to representing an actual earthquake than the filtered white-noise model since it consists of the sum of a series of independent impulses arriving at Poisson distributed times. The two models can be made identical up to the second moment, however, by imposing an impulse arrival rate $v$ that is a certain function of the white-noise spectral 
amplitude. Also, it can be shown that the white-noise models are Gaussian due to the Central Limit Theorem and the filtered Poisson process models, although in general nonGaussian, are asymptotically Gaussian as the impulse arrival $v \rightarrow \infty$ (Lin 1967). This non-Gaussian property can be used in applications where earthquake records indicate a significant deviation from Gaussian behavior.

\subsubsection{Spectral Representation Method}

The Spectral Representation Method is perhaps the most widely used approach to generate sample functions of a stochastic process, field or wave. Although the concept of the method has existed for some time (Rice 1944, 1945), it was Shinozuka and Jan (1972) and Shinozuka (1972) who first applied it for simulation purposes including multidimensional, multi-variate and non-stationary cases. In its simplest form, spectral representation simulates a $1 \mathrm{D}-1 \mathrm{~V}$ (one-dimensional and uni-variate) stationary stochastic process, which corresponds to seismic ground motion with a single horizontal component, using the following series:

$$
f(t)=\sqrt{2} \cdot \sum_{j=1}^{N} \sqrt{2 S_{X X}\left(\omega_{j}\right) \Delta \omega} \cdot \cos \left(\omega_{j} t+\phi_{j}\right)
$$

where $S_{X X}(\omega)$ is the known one-sided power spectral density of the stochastic process, $\Delta \omega$ is the frequency interval used to discretize the power spectrum and $\phi_{j}$ are independent random phase angles uniformly distributed over the range $0 \rightarrow 2 \pi$. An upper cut-off frequency $\omega_{u}=N \Delta \omega$ is implied in Eq. [65] beyond which $S_{X X}(\omega)$ may be assumed to be zero for mathematical or physical reasons. Using this series, the auto-correlation function and expected value of the simulated process converge to those of $S_{X X}(\omega)$, and the process becomes asymptotically Gaussian by virtue of the Central Limit Theorem, as the number of terms $N$ increases. In addition, the simulated process is ergodic, at least to the second moment, regardless of the size of $N$. This makes the method directly applicable to time-domain analysis in which the ensemble average can be evaluated in terms of the temporal average. It should be noted that the sample functions generated using Eq. [65] 
will be periodic with the sampling period $T=2 \pi / \Delta \omega$. This periodicity may be eliminated by randomizing the frequencies $\omega_{j}$ either by adding a small random frequency $\delta \omega_{j}$ (Shinozuka and Jan 1972) or by considering the frequencies $\omega_{j}$ to be random variables with a probability density modeled after the spectral density of the process (Sólnes 1997). The latter technique automatically concentrates the random frequencies around the peaks in the spectral density of the stochastic process.

The extension of the $1 \mathrm{D}-1 \mathrm{~V}$ stationary case to a non-stationary stochastic process, to more realistically simulate seismic ground motion, may be accomplished by using the evolutionary power spectrum developed by Priestley $(1965,1967)$. In this case, the spectral representation series becomes:

$$
f(t)=\sqrt{2} \cdot \sum_{j=1}^{N} \sqrt{2 A^{2}\left(t, \omega_{j}\right) S_{X X}\left(\omega_{j}\right) \Delta \omega} \cdot \cos \left(\omega_{j} t+\phi_{j}\right)
$$

where $A\left(t, \omega_{j}\right)$ is the evolutionary modulating function and all other terms are the same as the stationary case given by Eq. [65]. Similar to the stationary case, the simulated process converges to the target evolutionary power spectrum and is asymptotically Gaussian as $\mathrm{N} \rightarrow \infty$. The process, however, is no longer ergodic since by definition only a stationary process can be ergodic. Also, it should be noted that the Fast Fourier Transform (FFT) technique developed by Cooley and Tukey (1965), which is used to determine the spectral amplitudes $S_{X X}\left(\omega_{j}\right)$ of the target process (Yang 1972), no longer applies when using an evolutionary power spectrum. However, for the special case when $A\left(t, \omega_{j}\right)=A(t)$, i.e. the modulating function is independent of frequency, the nonstationary stochastic process becomes a uniformly modulated non-stationary stochastic process and the FFT technique again applies. This situation is equivalent to modeling the non-stationary random process as a stationary random process modulated by a deterministic amplitude variation function as discussed previously. A more direct use of the FFT technique to generate sample functions of Gaussian random processes was proposed by Wittig and Sinha (1975), which substantially reduces computation time in comparison with using Eqs. [65] or [66]. In this method, discrete frequency functions 
that correspond to the Fourier transform of the target process are generated. Sample functions are then obtained by taking the inverse Fourier transform of the discrete frequency functions using the FFT technique.

The more complex cases of simulating an $\mathrm{nD}-1 \mathrm{~V}$ non-homogeneous stochastic field and an $\mathrm{nD}-\mathrm{mV}$ homogeneous stochastic field were also developed by Shinozuka and Jan (1972). A multi-variate stochastic field may be used, for example, to simulate different seismic ground motions at various locations in a large-scale structure (Kareem et al. 1997). The relationship between the stochastic processes at each location, which may be separately modulated, is defined by a coherence function, which is the frequency-domain equivalent of the time-domain based correlation function. Various researchers have extended the simulation of multi-variate stochastic fields to include among other things: non-Gaussian properties (Yamazaki and Shinozuka 1988), simulation of non-stationary vector processes using an FFT-based approach (Li and Kareem 1991), spatially incoherent ground motions (Ramadan and Novak 1993), and simulation of ground motion time-histories compatible with prescribed response spectra (Hao et al. 1989, Abrahamson 1993, Deodatis 1996, Zhang and Shinozuka 1996).

Even more complex non-stationary stochastic process models have been developed to simultaneously represent the amplitude and frequency non-stationarity of seismic ground motion. Frequency non-stationarity is due to the different arrival times of the P (primary or push) waves, S (secondary or shear) waves and surface (Rayleigh and Love) waves that propagate at different velocities through the earth's crust. Several studies have shown that non-stationarity in frequency content can have a significant effect on the response of both linear and non-linear structures (Yeh and Wen 1990, Papadimitriou 1990, Conte 1992). To account for this effect, a number of filtered whitenoise process based models, filtered Poisson process based models, and spectral representation based models have been proposed (Kubo and Penzien 1979, Safak and Boore 1986, Lin and Yong 1987, Grigoriu et al. 1988, Fan and Ahmadi 1990, Papadimitriou 1990, Yeh and Wen 1990, Conte and Peng 1997, Nakayama and Fujiwara 1997). 


\subsubsection{Stochastic Wave Theory}

Stochastic wave theory, which was developed by Deodatis and Shinozuka (1989), is an extension of the Spectral Representation Method. This technique attempts to more realistically simulate seismic ground motion by describing it as a stochastic wave arising from a propagating seismic wave. It is intended for seismic response analysis of largescale structures extending over a wide spatial area such as water and gas transmission systems and large-span bridges (Deodatis et al. 1990, Zhang et al. 1991).

\subsubsection{ARMA Models}

Auto-regressive moving average models are one of a family of stationary time series models that includes: auto-regressive (AR), moving average (MA), and mixed auto-regressive and moving average models (ARMA), as well as their extension to a particular class of non-stationary random processes, the auto-regressive integrated moving average model (ARIMA). A time series model is one in which a sequence of values are generated representing possible observations of a random process at discrete values of time. The model parameters are then estimated on the basis of a comparison of estimated statistics of the generated sequence and the statistics of the actual observations of the random process, which are treated as sample functions drawn out of an ensemble of infinite possibilities. This procedure is very similar to the filtered white-noise model in that the time series is generated by passing a discrete white-noise, which provides the required sequence of values from a random process, through a linear filter. The general form of a linear time series is given by (Nigam and Narayanan 1994):

$$
x_{t}=\mu+a_{t}+\psi_{1} a_{t-1}+\psi_{2} a_{t-2}+\ldots
$$

where $\mu$ and $\psi_{i}$ are fixed parameters. The series $\left(\ldots a_{t-1}, a_{t}, a_{t+1}, \ldots\right)$ is the white-noise sequence of identically distributed and independent random shocks with zero mean and constant variance $\sigma_{a}{ }^{2}$. In this form, the series $x_{t}$ is represented as the weighted sum of the 
current and past disturbances. Equation [67] may be rearranged, however, to express the time series in terms of the current disturbance and all previous observations of the process $x_{t}$ :

$$
x_{t}=\pi_{1} x_{t-1}+\pi_{2} x_{t-2}+\ldots+a_{t}+\delta
$$

where the weights $\pi_{i}$ are functions of the $\psi_{i}$ weights and $\delta$ is a constant which is a function of $\mu$ and $\psi_{i}$. The general linear process of Eq. [68] has an infinite number of terms but, for practical purposes, only a finite number of weighting terms are given a non-zero value. The auto-regressive (AR) model is given by Eq. [68] with $\pi_{i}=0$ for $i>$ p. The moving average (MA) model is given by Eq. [67] with $\psi_{i}=0$ for $i>\mathrm{q}$. The mixed auto-regressive moving average (ARMA) model of order $p, q$, denoted by ARMA $(p, q)$ is the sum of the AR and MA models given by:

$$
x_{t}=\pi_{1} x_{t-1}+\ldots+\pi_{p} x_{t-p}+a_{t}+\delta-\psi_{1} a_{t-1}-\ldots-\psi_{q} a_{t-q}
$$

where the negative sign in Eq. [69] is introduced by convention. The inclusion of both auto-regressive and moving average terms typically results in a model that has fewer terms than would be necessary for a model of pure AR or pure MA form. The variables in Eq. [69] are all scalar variables, which corresponds to a so-called single input-single output linear model. ARMA models may also be extended to multiple input-multiple output linear systems, in which case the observations $x_{t}$ and the random terms $a_{t}$ become vectors and the coefficients $\pi_{i}$ and $\psi_{i}$ become matrices.

The basic problem of modeling an observed time series $x_{t}$ by the ARMA model is the estimation of the coefficients $\left(\pi_{i}, \psi_{i}\right)$ and determining the best model order $(p, q)$ to fit the observed data. The estimation of parameters is usually based upon least squares or maximum likelihood methods (see Box and Jenkins 1970), while determination of the best choice for model order is commonly based on two criteria developed by Akaike (1979); the 'final prediction error' criterion (FPE) and the 'Akaike information criterion' 
(AIC). The application of ARMA models to simulate the observed time series of seismic ground accelerations, which are treated as single input-single output systems, was first developed by Liu (1970). Since that time a considerable volume of literature has been devoted to earthquake modeling using ARMA models, see for example Polhemus and Cakmak (1981), Chang et al. (1982), Safak (1989), and a review article by Kozin (1988).

The main advantage of ARMA models over the use of filtered white-noise process, filtered Poisson process or spectral representation models is the reduced computation time and computer memory requirements. The digital generation of sample functions of a random process is accomplished by recursively obtaining its sample values at discrete times once the model coefficients $\left(\pi_{i}, \psi_{i}\right)$ are estimated. The model requires only the generation of a sequence of independent Gaussian random variates $a_{l}$. For the simulation of structural response to the sample ground motion, only the storage of the appropriate model coefficients in the computer memory, the generation of the whitenoise sequence and the recursive computation of the time series is required. Another advantage of the method is that the time series can be generated in real time, which can be used in random vibration experiments.

\subsubsection{Wavelet Models}

The most recent development with applications to simulation of non-stationary multi-variate processes is the use of wavelet functions, which were initially developed for analyzing seismic data in oil exploration studies. For engineering purposes, Newland (1994a, 1994b) applied Daubechies' wavelet (Daubechies 1992) for analyzing vibration signals and developed Discrete Wavelet Transform (DWT) and Fast Wavelet Transform (FWT) computational algorithms, which have emerged as powerful tools to analyze the temporal variations in frequency content of non-stationary processes.

For purposes of modeling seismic ground acceleration, the wavelet representation of a zero mean process $f(t)$ with non-stationary characteristics is summarized as follows (Basu and Gupta 1997, 1998): 


$$
\begin{aligned}
& W_{\psi} f(a, b)=\int_{-\infty}^{\infty} f(t) \psi_{a, b}(t) d t, \rightarrow a>0 \\
& f(t)=\frac{1}{2 \pi C_{\psi}} \int_{-\infty-\infty}^{\infty} \int_{-\infty}^{\infty} \frac{1}{a^{2}} W_{\psi} f(a, b) \psi_{a, b}(t) d a d b \\
& \psi_{a, b}(t)=\frac{1}{|a|^{1 / 2}} \psi\left(\frac{t-b}{a}\right) \\
& C_{\dot{\psi}}=\int_{-\infty}^{\infty} \frac{\mid \hat{\psi}(\omega)^{2}}{|\omega|} d \omega<\infty \\
& \hat{\psi}(\omega)=\frac{1}{\sqrt{2 \pi}} \int_{-\infty}^{\infty} \psi(t) e^{-i \omega t} d t
\end{aligned}
$$

where the parameter $b$ has the physical significance of localizing the wavelet basis function $\psi(t)$ at $t=b$ and the parameter $a$ captures the local frequency content. For numerical evaluation of the integrals in Eq. [70], discretization parameters $\sigma$ and $\Delta b$ are used, resulting in $a_{j}=\sigma^{j}$ and $b_{j}=(j-1) \Delta b$. The step changes at $a=a_{j}$ and $b=b_{j}$ are defined as:

$$
\begin{aligned}
& \Delta b_{j}=\frac{\left(b_{j+1}-b_{j}\right)+\left(b_{j}-b_{j-1}\right)}{2}=\Delta b \\
& \Delta a_{j}=\frac{\left(a_{j+1}-a_{j}\right)^{2}+\left(a_{j}-a_{j-1}\right)}{2}=\frac{a_{j}}{2}\left(\sigma-\frac{1}{\sigma}\right)
\end{aligned}
$$

The discretized version of the time-history of the ground acceleration is then given by:

$$
\begin{aligned}
& f(t)=\sum_{i} \sum_{j} \frac{K \Delta b}{a_{j}} W_{\psi} f\left(a_{j}, b_{i}\right) \psi_{a_{j} b_{i}}(t) \\
& K=\frac{1}{4 \pi C_{\psi}}\left(\sigma-\frac{1}{\sigma}\right)
\end{aligned}
$$


The choice of the wavelet basis function $\psi(t)$ that should be used to model $f(t)$ in the above equations primarily depends on how suitable a given basis function is for the dynamic system. The referenced papers (Basu and Gupta 1997, 1998) used a slightly modified form of the Littlewood-Paley (L-P) basis function given by:

$$
\psi(t)=\frac{1}{\pi \sqrt{\sigma-1}} \frac{\sin \sigma \pi t-\sin \pi t}{t}
$$

The application of wavelet-based functions can be extended to a wavelet - based random vibration theory to predict the stochastic dynamic response statistics of a linear time-invariant system. This is exactly analogous to the well-developed linear random vibration methods in the frequency domain. 


\subsection{HYSTERESIS MODELS}

Response analysis of any dynamic system, whether linear and deterministic or non-linear and stochastic, requires a model that governs the relationship between input and response. For dynamic mechanical and structural systems undergoing inelastic deformation, this relationship is given by the equation of motion, which includes a term expressing the hysteretic restoring force as a function of system displacement. This hysteretic restoring force depends not only on the instantaneous displacement of the system, but also on the time history of the response and it may deteriorate in strength, stiffness or both as dynamic oscillation progresses. Models describing this hysteretic, time-dependent behaviour of yielding dynamic systems are classified as either phenomenological or mechanics-based. Phenomenological models describe the nature of the load-deformation relationship based on observations but do not necessarily explain the behaviour, while mechanics-based models are based on the properties of the individual elements that comprise the system. Mechanics-based models provide better insight into how material properties affect system response but they are inconvenient to use in inelastic dynamic analysis of complete structural systems made up of multiple members (Foliente et al. 1998).

\subsubsection{General Hysteresis Models}

Early hysteresis models to describe inelastic structural behaviour were phenomenological and typically bilinear, including the well-known elastoplastic model and the Ramberg-Osgood model. The Ramberg-Osgood (1943) model describes the force-displacement curve envelope curve (or backbone curve or skeleton curve) by a three-parameter polynomial, and allows smooth transition from the elastic to the plastic region and some freedom in the shape of the hysteresis. However, as in the case of the bilinear model, it was difficult to include system deterioration in the model. Later models proposed by Clough and Johnston (1966), Takeda et al. (1976) and others, extended the basic bilinear system to include deterioration by using a set of empirical rules. The use of piece-wise linear models of the force-displacement relationship, governed by a set of 
empirical stiffness rules related to displacement, is well-suited for time-history analysis of structural response by means of step-by-step numerical integration. This has resulted in a wide variety of models being developed for various materials and types of loading (see, for example, the 3-D non-linear dynamic structural analysis program $C A N N Y 99$ (Li 1996), which has twenty uni-axial hysteresis models). However, for analytical treatment of system response to random vibration, the use of empirical rules is difficult to put in a mathematically tractable form and, therefore, non-linear differential equations are used to model the force-displacement relationship.

Differential equation models are still phenomenological in nature but they are given in a mathematically explicit form so that an analytical solution of the system response may be obtained. The first smooth hysteretic restoring force model was proposed by Bouc (1967) and later generalized by Wen (1976), however, this model did not include the pinching and degradation behaviour exhibited by many hysteretic systems that sustain damage under large deformation. For this reason, extensions to the model were proposed by Baber and Wen (1981) who incorporated stiffness and/or strength degradation as a function of hysteretic energy dissipation, and Baber and Noori (1986) who incorporated pinching behaviour using a 'slip-lock' element. This model, which has since become known as the Bouc-Wen-Baber-Noori (BWBN) hysteresis model, introduces a state variable $z$ and separates the restoring force into non-hysteretic and hysteretic components. For a single-degree-of-freedom (SDOF) non-linear system, the equation of motion in standard form is then given by:

$$
\ddot{u}+2 \xi \omega_{0} \dot{u}+\alpha \omega_{0}^{2} u+(1-\alpha) \omega_{0}^{2} z=f(t)
$$

where $\xi$ is the system damping ratio, $\omega_{0}$ is the natural frequency, $\alpha$ is the ratio of postyielding to pre-yielding stiffness, $f(t)$ is the mass normalized forcing function, typically assumed to be zero mean, and $z$ is the 'hysteretic force', which is described by the nonlinear differential equation: 


$$
\dot{z}=h(z) \cdot\left[\frac{A \dot{u}-v\left(\beta|\dot{u} \| z|^{n-1} z-\gamma \dot{u}|z|^{n}\right)}{\eta}\right]
$$

The parameters $A, \beta \gamma$ and $n$ are the hysteresis shape parameters (if $n=\infty$, the bilinear elastoplastic case is obtained), $v$ and $\eta$ are the strength and stiffness degradation parameters, and $h(z)$ is the pinching function introduced by Baber and Noori (1986). Note that for systems in which the loading fluctuates in such a manner that the displacement is cyclic but does not change sign, there is a tendency of the BWBN model to introduce some artificial drift into the system response. This problem can be corrected by adding two more terms to the governing equation (Casciati 1987) but it is not particularly important for systems undergoing random excitation (Wen 1989).

The SDOF system response model given by Eqs. [74] and [75] states that the rate of increase of the restoring force depends on the state of the system (in terms of $u$ and $z$ ) as well as whether it is in a loading or unloading stage, due to the absolute value signs. Also, for a given time-history of displacement, the restoring force is completely specified by the differential equation i.e. there are no empirical rules. However, the model also forces pinching to occur at zero load, which doesn't necessarily reflect the true behaviour of all structural systems, some of which pinch at a residual force level. To generalize the BWBN model, Foliente (1995) added an additional constant parameter $q$ that sets the pinching level as a fraction of the ultimate value of $z$. This model is referred to as the modified BWBN model and is given by the following:

$$
h(z)=1-\xi_{1} \exp \left[-\frac{\left(z \operatorname{sgn}(\dot{u})-q z_{u}\right)^{2}}{\xi_{2}{ }^{2}}\right]
$$

where $\operatorname{sgn}\left(\mathrm{)}\right.$ is the signum function, and $z_{u}$ is the ultimate value of $z$ given by:

$$
z_{u}=\left[\frac{1}{v(\beta+\gamma)}\right]^{\frac{1}{n}}
$$


and the parameters that control the severity and rate of pinching, respectively, are:

$$
\begin{aligned}
& \xi_{1}=\xi_{1 o}[1-\exp (-p \varepsilon)] \\
& \xi_{2}=\left(\psi_{0}+\delta_{\psi} \varepsilon\right)\left(\lambda+\xi_{1}\right)
\end{aligned}
$$

Strength and stiffness degradation are modeled, respectively, by:

$$
\begin{aligned}
& v=1+\delta_{v} \varepsilon \\
& \eta=1+\delta_{\eta} \varepsilon
\end{aligned}
$$

Finally, strength and stiffness degradation, as well as pinching, are controlled by the hysteretic energy dissipation given by the following:

$$
\varepsilon=(1-\alpha) \omega_{0}^{2} \int_{t_{0}}^{t_{s}} z \dot{u} d t
$$

The modified BWBN model, which has been extended to bi-axial hysteretic systems (Park et al. 1986), is very flexible and can produce a wide variety of hysteresis shapes to model the behaviour of hysteretic degrading systems. In addition, standard damping ratios for linear systems can be used for response analysis since the BWBN model simply adds a hysteretic element to a mechanical model that contains linear viscous damping and a linear spring. Once the parameters of the hysteresis model are identified, dissipated energy can be obtained from the hysteresis trace of the response. The identification or estimation of suitable model parameters for a particular set of system materials and configurations is known as a system identification problem. A simple technique to determine the parameters from test or field data, which is based on least square minimization, was developed for the uni-axial and bi-axial BWBN model by Wen and Ang (1987) and Sues et al. (1988). Other common methods, which were cited 
by Foliente et al. (1998), include: sequential regression analysis (Masri and Caughey 1979, Masri et al. 1982, Masri et al. 1987), spectral method (Roberts et al. 1995), Newton's method, Gauss' method, and the extended Kalman filtering technique. The general theoretical aspects of hysteretic system identification have been reviewed by Minai and Suzuki (1987).

Recently, Dobson et al. (1998) presented a Boolean model for mechanical hysteretic systems that uses a set of Boolean statements to provide a piece-wise nonlinear representation of the loading and unloading curves over an arbitrary number of sub-intervals. By significantly increasing the number of sub-intervals, the modeling of hysteresis can be accomplished by using a database of arrays, which eliminates the need for system identification when the hysteresis loop is constructed from experimentally sampled static or dynamic data. 


\subsection{SEISMIC STRUCTURAL RESPONSE AND RELIABILITY STUDIES}

The assessment of structural response under dynamic loading has received considerable attention in the literature over the past 30 years. With the advent of increasingly powerful personal computers, researchers are now able to conveniently analyze complex analytical random vibration models or quickly perform time-history analysis, which has led to a wealth of literature relating to structural response under dynamic loading. Considering only those studies that relate to non-linear structural response under seismic loading, a very brief review of some past work that has utilized one or several of the analytical or numerical techniques summarized in Sections $2.2-2.4$ is given below. This review does not list those studies that include a structural response analysis to illustrate the use of a proposed analytical or numerical technique. Those studies have already been cited in the relevant section describing the technique.

A large majority of the literature deals with seismic response analysis of steel and concrete structures, some of which were considered deterministic and others that were treated as having variable mechanical properties. These studies are listed in order of the type of analysis that was used beginning with the Monte Carlo method, which seems to be the most popular non-linear stochastic dynamic analysis technique. Liu (1969) used fifty artificial earthquake records based on the 1940 El Centro earthquake and elasto-plastic and bilinear degrading hysteresis loops to collect SDOF earthquake response statistics; Meskouris and Kratzig (1987) used three records and a Takeda-type hysteretic law to investigate a five-storey concrete frame; O'Connor and Ellingwood (1987) used 20 California earthquake records in a Latin Hypercube Sampling scheme and an elastoplastic hysteresis loop to determine SDOF steel frame reliability indices based on limit states defined by displacement ductility and a low-cycle fatigue damage index; Conte et al. (1991) used two sets of 100 ARMA model records and several piece-wise linear hysteresis models to evaluate SDOF response statistics and perform a sensitivity study on structural parameters; Seya et al. (1993) used eighteen artificial records in a Latin Hypercube Sampling scheme and a bilinear hysteresis loop to generate fragility curves for five displacement ductility-based limit states for a hypothetical five-storey steel 
building; Bolotin (1993) used twenty artificial records to investigate the effect of random peak ground accelerations and spectral characteristics as well as random structural parameters on the response of a sixteen-story building; Lee (1996) used six California records and a bilinear hysteresis loop to investigate the significance of peak ground acceleration to peak ground velocity ratio on the displacement ductility demand of a tenstorey frame structure; Collins et al. (1996) used a uniform hazard spectra approach based on 1292 artificial records generated for the Los Angeles area to evaluate structural performance of SDOF frames using displacement ductility-based limit states. This work included a proposed reliability-based seismic design procedure using displacementbased performance criteria and an equivalent system methodology for application to MDOF structures; Han and Wen (1997) used 88 records recorded around the world and a proposed 'equivalent non-linear system' approach to evaluate the performance of seven multi-storey structures; Marek et al. (1997) performed a sensitivity analysis on structural random variables using 100 artificial records in a Latin Hypercube Sampling scheme; Bagchi (1999) used four records generated by the Geological Survey of Canada from uniform hazard spectra to evaluate the performance of concrete frame structures in Victoria and Montreal.

The other methods of analysis have not been used nearly as frequently as the Monte Carlo technique. Suzuki and Araki (1997) used the Response Surface Method to evaluate the reliability of a two-storey frame structure with random structural properties using the $1940 \mathrm{El}$ Centro and 1995 Kobe earthquake records and a bilinear hysteresis loop; Casciati and Faravelli (1985) also used the Response Surface Method to generate seismic fragility curves for a four-storey frame structure with random structural properties. Several other authors have used the analytical Equivalent Linearization Method to evaluate structural performance: Sues et al. (1985) analyzed a four-storey steel frame building and a seven-storey concrete building using the BWBN hysteresis model and random structural properties; Wen and Eliopoulos (1994) analyzed a fivestorey frame to determine the response statistics and then used the statistics to calculate probabilities of exceeding certain inter-storey drift limits given a certain seismic hazard; Foliente et al. (1996) evaluated the accuracy of Equivalent Linearization incorporating 
the modified BWBN model against 200 Monte Carlo response samples and then analyzed the sensitivity of the response to changes in structural properties and hysteresis shape parameters. Finally, a number of authors have used stochastic finite element solutions to determine the reliability of structures under dynamic loads (Langley 1985, Mahadevan and Mehta 1991, Faravelli 1992, Bucher and Brenner 1992, Brenner and Bucher 1996).

Research on the seismic performance of timber structural systems has not received the same attention in the literature as that for steel and concrete structures. This may be due to its less common usage in large-scale structures and perhaps because of the difficulty in characterizing timber structural behaviour, which besides being non-linear may also be influenced by the rate and duration of loading. Of the research that has been done, some of the recent work is summarized below.

Chui and $\mathrm{Ni}$ (1995) investigated the effect of the moment-rotation characteristics of circular bolted timber connections on the seismic performance of a timber frame. Using a DRAIN-2D moment-rotation hysteresis model, the frame response to the 1940 El Centro and 1971 Orion Blvd. earthquakes for a range of hysteresis model parameters was determined. The study showed that very stiff connections likely result in brittle failure of the timber members, while normally stiff connections would result in a connection failure at large displacement. The shape of the hysteresis loop was also shown to influence response levels, with highly pinched loops resulting in larger displacement responses. Latendresse et al. (1995) used a mechanics-based finiteelement approach to model multiple dowel connections using the analogy of a beam on a deformable foundation. Experimental hysteresis loops under cyclic loading were shown to match well with the analytical model. Yasumura (1996) performed a series of cyclic lateral load tests on wood framed shear walls, glulam braced frames, glulam moment resisting frames and glulam arched frames to determine a 'behaviour' factor $q$, which is a function of the displacement ductility. It appears that the behaviour factor is identical to the well-known force reduction factor $R$ in the 1995 NBCC, since it is the same function of ductility as that proposed by Newmark and Hall (1973) i.e. $q=R=\sqrt{ }(2 \mu-1)$. Using the behaviour factor, a timber structural system of each type was designed and tested using 
the $1940 \mathrm{El}$ Centro, 1952 Taft and 1995 Kobe earthquakes to evaluate its seismic performance. Results showed that the maximum displacement response was usually within the collapse limit of each structure. Frenette et al. (1996) tested a two-storey Parallam ${ }^{\circledR}$ moment resisting frame using six consecutive earthquake records, which were taken from the 1992 Landers earthquake and scaled to different peak ground accelerations. The purpose was to assess the elastic characteristics of the frame and then observe its performance during a major earthquake, an aftershock and subsequent major earthquakes. Experimental results compared well with analytical predictions using DRAIN-2DX and DPSA, a material properties based non-linear finite element program. Foliente et al. (1998) summarized the system identification process for estimating the parameters in the modified BWBN hysteresis model and then used experimental data from shear wall pseudo-dynamic tests in Japan to estimate the parameters. Using the 1989 Loma Prieta and 1995 Kobe earthquakes, a comparison was made between the time history response using a non-degrading, non-pinching hysteresis model and the timehistory response using the modified BWBN model. It showed that displacement response might be severely underestimated if a non-degrading, non-pinching hysteresis model is used. Ceccotti and Karacabeyli (1998) evaluated the required 'Action Reduction Factor' for moment resisting timber frames, which is analogous to the force reduction factor $R$ in the 1995 NBCC. Using eight earthquake records mainly from Italy, it was determined that an ARF of 2 is appropriate for one-storey buildings with timber moment resisting frames. Hockey et al. (1999) summarized several techniques for the reinforcement of timber connections to increase ductility for earthquake resistance. These techniques included: fibreglass reinforcement, glued plywood reinforcement, application of a truss connector plate, and the insertion of glued-in or threaded rods transverse to the wood grain. Popovski et al. (1999) evaluated the dynamic response of braced timber frames with various brace connections. Using DRAIN-2DX, with a hysteresis model based on experimental shake table tests, a time-history analysis was done using five earthquake records from around the world. From these analyses, the influence of different connection details on the seismic response of braced timber frames was determined. In addition, a conservative force reduction factor $R$ of 1.5 was suggested for bolted connections, while for glulam riveted connections an $R$ value of 2.0 was suggested. 
Finally, Karacabeyli and Ceccotti (1999) evaluated the dynamic performance of a fourstorey wood shear wall structure using 28 earthquake records, six of which were real records and the remaining 22 were records that had been modified to fit the Vancouver area design spectrum. Results showed that the current 1995 NBCC force modification factor $R=3$ for plywood nailed shear walls is appropriate. 


\section{CHAPTER 3}

\section{NUMERICAL ALGORITHM DEVELOPMENT}

\subsection{INTRODUCTION}

The development of a software application to enable more rigorous structural reliability analysis under seismic loading began by selecting a suitable probabilistic analysis method from among the available methods outlined in Chapter 2. Once the decision was made to use the numerical analysis approach, the task of outlining the basic structure of the software and deciding what functionality should be included was the next step in the process of creating and integrating the required algorithms. Chapter 3 begins with a brief summary of the reasons for adopting the numerical analysis approach followed by several sections that detail key numerical components, existing model modifications and solution methods that form the foundation of the chosen structure and functionality of PSResponse. In each section, relevant computational issues such as; running time, error control, convergence, proper numerical estimation and random number generation are discussed.

It should be noted that the details and discussion in each of the following sections, and the sections themselves, are not intended to be a complete description of the algorithmic structure of PSResponse (see Chapter 4). The intent of this chapter is to provide the important technical details behind the algorithms that form the software.

\subsection{SELECTION OF ANALYSIS METHOD}

The decision regarding which probabilistic analysis method should be used to determine the statistical dynamic response of structures undergoing earthquake induced excitation was based on the key requirements of robustness and flexibility. The chosen 
analysis method had to allow for significant complexity in the structural system for analysis of multi-storey structures, while also allowing for a large degree of nonlinearity in system restoring forces to accommodate the hysteretic nature of inelastic structural seismic response. In addition, the stochastic input excitation, in the form of earthquake induced ground acceleration, should reflect the true nature of the random excitation process, rather than a mathematically tractable idealization. These requirements tended to eliminate all the frequency domain based analytical methods, with the exception of the Equivalent Linearization Method, due to their various serious limitations regarding the nature of the restoring force, structural complexity or type of random excitation process.

The Markov based methods each required that the input excitation be a stationary Gaussian process (i.e. white noise), which is a poor representation of a real earthquake spectrum, and most of the methods (Galerkin Method, Finite Element Method, Closure Technique) have the disadvantage of slow convergence or large computational requirements for highly non-linear or multiple-degree-of-freedom systems. In the case of the Numerical Diffusion Method, the applicability of Generalized Cell Mapping (GCM) to degrading hysteretic systems, which characterizes most structural systems, is not known.

Other analytical methods of determining the statistical response of structures to stochastic excitation, such as the Perturbation Method and the Functional Series Representation Method, are not confined to a white-noise assumption for the excitation and are even applicable to multiple-degree-of-freedom systems. However, each of these methods are limited to weakly non-linear systems and as such are not applicable to ductile, hysteretic structural systems.

The Equivalent Linearization Method has been successfully applied to response and damage prediction of a variety of highly non-linear structural systems under seismic excitation. However, response results may tend to be underestimated using this method when the excitation spectral content is such that the power spectral density function 
vanishes rapidly as the frequency goes to zero, which is typical of earthquake excitation. In addition, the assumption of a Gaussian input excitation results in an assumed Gaussian response of the linearized non-linear system. This assumption is not correct for a nonlinear system, which is known to have a non-Gaussian response to a Gaussian input. The result of an assumed Gaussian response is that it may significantly misrepresent the frequency of high response levels to extreme loads, which contribute most to firstpassage and fatigue failures.

With these limitations in mind, the decision to forego the frequency domain based methods in favour of a time-domain based approach was made. The time-history approach to finding the response statistics of a dynamic system is generally more accurate and robust than any of the analytical random vibration methods since there are no limitations or assumptions required to obtain response statistics using time domain based numerical methods. This generality is the reason that numerical methods are frequently used to verify results obtained using other analytical random vibration methods.

\subsection{NUMERICAL MODELS AND SOLUTION METHODS}

Having chosen to use a time-history approach for probabilistic dynamic response analysis, the development of algorithms to solve the general differential equation of motion for random earthquake loading required numerical models and solution methods in four major areas: earthquake generation, hysteresis modeling, structural modeling and an overall numerical time-stepping method. The noteworthy technical details in each major area are summarized in the following.

\subsubsection{Numerical Time-Stepping Method}

Since analytical solution of the equation of motion is not possible for arbitrarily varying excitations and non-linear systems, a numerical time-stepping method is required to integrate the differential equation, or system of equations, governing the 
response of the system. There are a number of types of time-stepping procedures applicable to both linear and non-linear systems that are categorized by the assumption made in interpolating the system response and their inherent stability. The stability of a numerical procedure refers to its ability to return a bounded solution for a given timestep length. Procedures that "blow up" in the presence of numerical round-off and return meaningless results if the time-step is longer than some stability limit are conditionally stable, while those that lead to bounded solutions regardless of the time-step length are unconditionally stable.

For response analysis of single-degree-of-freedom (SDOF) systems, whether linear or non-linear, the stability criterion does not usually control the choice of time-stepping procedure since the time--step required for numerical accuracy is considerably smaller than the stability limits of the conditionally stable procedures. However, for response analysis of multiple-degree-of-freedom (MDOF) systems, unconditionally stable procedures are generally necessary to avoid the excessive computational demands of conditionally stable procedures, which require an extremely short time-step to remain within the stability limit of higher modes of response. Since MDOF analysis capability was a definite requirement of the software application, an unconditionally stable timestepping procedure was needed. Selection of which procedure to use for algorithm development was based on a review of time-stepping procedures (Chopra 1995) that compared the solution results for a linear system using two procedures; Newmark's Average Acceleration Method and Wilson's Method. That review showed that Wilson's Method introduces numerical damping into the dynamic system, whereby the system displacement amplitude decays with time even in the absence of system damping. No amplitude decay is introduced by Newmark's Average Acceleration Method. Also, the tendency for numerical methods to elongate or shorten the natural period of response is reduced in the Newmark Method as compared to Wilson's Method, both of which induce a period elongation. In addition, a second Newmark procedure called the Linear Acceleration Method, which is conditionally stable, induces an even smaller period elongation than the Average Acceleration Method and is, therefore, more accurate than its unconditionally stable counterpart. For this reason, using the Linear Acceleration 
Method is preferred over the other methods in situations where a conditionally stable procedure is allowable.

With these properties in mind, both Newmark methods were selected for response analysis. This was done because there is no difference in the time-stepping algorithm between the two methods except for the value of two constants, $\gamma$ and $\beta$. Therefore, the superior accuracy of the Linear Acceleration Method could be utilized and provision made to switch to the Average Acceleration Method when required for stability reasons.

\subsubsection{Time-Stepping Overshoot Problem}

Integration of system response in a time-stepping procedure can give rise to significant error when the transitions in the force-deformation relationship associated with velocity sign changes are not followed closely. When the calculated response velocity $\mathrm{v}_{\mathrm{i}+1}$ at time $i+1$ changes sign with respect to velocity $\mathrm{y}_{\mathrm{i}}$ this indicates that at some point during the time-step the response displacement either started increasing or decreasing. If the point at which the velocity went to zero during the time-step is not identified in the numerical procedure then the step-by-step path of the force-deformation relationship will "overshoot" the true path and the calculated displacement at the end of time-step $i+1$ will be either too large or too small. These departures from the exact path will occur at each reversal of velocity, leading to errors in the numerical results.

To minimize the overshoot problem, the Newmark time-stepping algorithm uses a variable time-step when velocity sign changes are detected. The sign of the velocity is tested after each time-step and in the event of a sign change the integration is reset back to the beginning of the time-step. The time-step is then repeatedly bisected until the absolute value of the response velocity at the end of the reduced time-step is less than a preset fraction of the peak response velocity. The peak response velocity is assumed to be $10 \%$ of the peak ground velocity, which is conservative except for extremely short and extremely long natural period structures, based on a typical response spectrum. Assuming a peak response velocity based on peak ground velocity, rather than actually 
calculating it, is done to eliminate the need to determine the peak response velocity from an initial guess of an appropriate minimum velocity and then iteratively adjusting the minimum velocity to be a fraction of the updated value of peak response velocity.

\subsubsection{Structural Model}

The type of structures that the software application is intended to model are those that may be represented as typical lumped mass idealizations consisting of shear walls or frames as the lateral load resisting elements. These idealized structures are further simplified to lateral-degree-of-freedom only models prior to response analysis to facilitate rapid calculation of the response time-history. Also, calculation of the displacement time-history is based solely on integration of the governing differential equation of motion, consequently, the second-order effect on lateral displacement produced by the vertical load acting on the structure in its displaced configuration is not considered in the displacement calculation.

The mathematical representation of any type of structure is contained in its mass and stiffness matrices. For both types of structures considered in the software application, categorized as shear and frame, the mass matrix is diagonal owing to the assumption of lumped floor masses. Shear structures are considered to have rigid horizontal beams, slabs or diaphragms, which eliminates all rotational degrees of freedom and renders the stiffness matrix a lateral stiffness matrix automatically. Frame structures retain the rotational degrees of freedom at the intersection of horizontal and vertical elements since the horizontal elements are not considered rigid. The stiffness matrix is then reduced to a lateral stiffness matrix by eliminating the rotational degrees-offreedom through static condensation since it is assumed that no mass is associated with the rotational degrees-of-freedom. The static condensation process employs an LU decomposition and back-substitution procedure for the required matrix inversions. 
For modal analysis and information purposes, the natural frequencies of the structure are determined by solving the structural dynamics eigenvalue problem given by the following:

$$
\mathbf{k} \phi=\omega^{2} \mathbf{m} \phi
$$

The eigensystem in Eq. [1] is solved by first transforming it into the standard eigenvalue problem by pre-multiplying by $\mathbf{m}^{-1}$. This gives:

$$
\mathbf{A} \phi=\lambda \phi
$$

where $\mathbf{A}=\mathbf{m}^{-1} \mathbf{k}$ and $\lambda=\omega^{2}$. In general, $\mathbf{A}$ is not symmetric although $\mathbf{m}$ and $\mathbf{k}$ are both symmetric matrices. The eigensystem is then solved using a Jacobi procedure that requires that $\mathbf{A}$ be a symmetric matrix, which is achieved by

$$
\mathbf{A y}=\lambda \mathrm{y}
$$

where $\mathbf{A}=\mathbf{m}^{-1 / 2} \mathbf{k m}^{-1 / 2}$ and $\mathrm{y}=\mathbf{m}^{-1 / 2} \phi$ and $\lambda=\omega^{2}$.

The mode shapes $\left(\phi_{n}\right)$ associated with the natural frequencies $\left(\omega_{n}=\sqrt{ } \lambda_{n}\right)$ are determined by pre-multiplying the eigenvector matrix $\left(y_{n}\right)$ by $\mathbf{m}^{-1 / 2}$. The resulting mode shapes are normalized such that the generalized modal masses $\mathbf{M}_{n}$ are 1 . $\mathrm{Re}-$ normalization of the mode shapes to 1 is straightforward.

The distribution of damping in the structural model is determined by whether the system is linear or non-linear. Classical damping is assumed for linear systems to allow for modal analysis. For non-linear systems, damping may be specified as Rayleigh damping or as a multiple of a single baseline damping value for each storey. 


\subsubsection{Hysteresis Model}

Modeling of the hysteretic restoring force in the equation of motion is done using the Bouc-Wen-Baber-Noori (BWBN) hysteresis model (Sec. 2.4.1), which was modified to reduce the number of parameters that need to be identified (see Sec. 3.3.4.4). The BWBN model is used because it is able to produce a wide variety of hysteresis shapes, including the pinching and degradation behaviour exhibited by many hysteretic systems, without the use of piece-wise linear equations governed by numerous empirical rules relating stiffness to displacement.

\subsubsection{Modification to the BWBN model}

The BWBN hysteresis model introduces a state variable $z$ into the equation of motion and separates the restoring force into non-hysteretic and hysteretic components. For an SDOF system, the equation of motion in standard form is then given by:

$$
\ddot{u}+2 \xi \omega_{0} \dot{u}+\alpha \omega_{0}{ }^{2} u+(1-\alpha) \omega_{0}{ }^{2} z=f(t)
$$

where $\xi$ is the system damping ratio, $\omega_{0}$ is the natural frequency, $\alpha$ is the ratio of postyielding to pre-yielding stiffness, $f(t)$ is the mass normalized forcing function and $z$ is the hysteretic displacement, which is described by the non-linear differential equation:

$$
\dot{z}=h(z) \cdot\left[\frac{A \dot{u}-v\left(\beta|\dot{u} \| z|^{n-1} z-\gamma \dot{u}|z|^{n}\right)}{\eta}\right]
$$

where the parameters $A, \beta \gamma$ and $n$ are the hysteresis shape parameters, $v$ and $\eta$ are the strength and stiffness degradation parameters, and $h(z)$ is the pinching function given by the following: 


$$
h(z)=1-\xi_{1} \exp \left[-\frac{\left(z \operatorname{sgn}(\dot{u})-q z_{u}\right)^{2}}{\xi_{2}^{2}}\right]
$$

where $\operatorname{sgn}(\mathrm{)}$ is the signum function, $q$ is a constant parameter that sets the pinching level as a fraction of the ultimate value of $z$, and $z_{u}$ is the ultimate value of $z$. The parameters that control the severity and rate of pinching, respectively, are:

$$
\begin{aligned}
& \xi_{1}=\xi_{1 o}[1-\exp (-p \varepsilon)] \\
& \xi_{2}=\left(\psi_{0}+\delta_{\psi} \varepsilon\right)\left(\lambda+\xi_{1}\right)
\end{aligned}
$$

Finally, strength and stiffness degradation are modeled, respectively, by:

$$
\begin{aligned}
& v=1+\delta_{\nu} \varepsilon \\
& \eta=1+\delta_{\eta} \varepsilon
\end{aligned}
$$

where $\varepsilon$ is the dissipated hysteretic energy.

In total there are thirteen separate parameters in the BWBN model that must be identified, although two of the parameters, $A$ and $n$, are typically set to 1 . The identification or estimation of suitable model parameters for a given structural configuration and material type is known as a system identification problem, which rapidly increases in difficulty as the number of parameters increases. To simplify the system identification problem the existing pinching function, which utilizes six parameters, was modified to have only three parameters. The remaining non-unity parameters; $\alpha, \beta, \gamma, \delta_{\nu}$ and $\delta_{\eta}$ are more fundamental and were not altered. The revised pinching function is given by:

$$
h(u, z)=f \cdot\left[1+\mu \cdot\left[\frac{1}{f}-1\right] \cdot[(\beta+\gamma) \cdot z]^{2}+v \cdot[1+\operatorname{sgn}(u) \cdot \operatorname{sgn}(\dot{u})] \cdot[1-f] \cdot[10 \cdot(\beta+\gamma) \cdot u]^{2}\right]
$$


where $f=\exp (-\phi \cdot \varepsilon)$ and $\operatorname{sgn}()$ is the signum function as before. In this modified pinching function the role of each of the three new parameters; $\varphi, \mu$ and $v$, is more easily understood than the relationship between the six parameters of the original pinching function. The parameter $\varphi$ controls the overall rate of increase in pinching as damage cycles progress, the parameter $\mu$ controls the rate of stiffness recovery throughout the loading phases of each cycle and the parameter $v$ controls the rate of stiffness recovery during the increasing displacement portion of each loading phase.

In modifying the pinching function the assumption was made that pinching occurs at zero restoring force, which is equivalent to the assumption that $q=0$ in the original pinching function. Therefore, in effect, the pinching function has been reduced from a five parameter model to a three parameter model in its modified form. The assumption that pinching occurs at or very near zero restoring force was based on the observation that significant hysteretic pinching in overall cyclic structural behaviour is largely the result of structural damage associated with localized failures such as cracking and connection degradation due to material crushing. This structural damage decreases the initial stiffness at the very beginning of each loading cycle, until increasing displacement closes the cracks and connection gaps at which point stiffness begins to increase. Individual fasteners and reinforcement within the structure may continue to exhibit typical yielding behaviour where stiffness at the beginning of each loading cycle is equivalent to the elastic stiffness until yield is reached; however, the overall behaviour of a structure includes the cumulative effect of damage throughout the entire structure, which degrades initial stiffness at the outset of each loading cycle.

Figure 3.1 shows a comparison of the original and modified pinching functions in the BWBN model for three types of cyclic behaviour corresponding to a structure subjected to an increasing amplitude sinusoidal displacement. The first two cases assume that pinching occurs at zero restoring force, with subsequent stiffness recovery or no stiffness recovery (Fig. 3.1, top and middle), while the third case illustrates pinching at a non-zero restoring force (Fig. 3.1, bottom). The hysteretic data was generated by 
selecting the original parameters of the BWBN model such that the structure undergoing the sinusoidal displacement exhibited significant yielding and stiffness degradation behaviour and then the modified pinching function parameters were fitted to that hysteresis loop. From Figure 3.1 it can be seen that the modified pinching function provides a good fit to the original data when pinching occurs at zero restoring force, however, as expected, it increasingly underestimates the hysteretic force at the beginning of a loading cycle as the cycles progress when pinching occurs at a non-zero restoring force. Therefore, response displacement may tend to be overestimated using the modified pinching function in applications that exhibit pinching behaviour at a significant force.

\subsubsection{Numerical Solution of Hysteresis Model}

Determining the hysteretic displacement $z$ in the differential equation of motion at each time-step in the Newmark procedure requires solution of Eq.[5], which is a firstorder, non-linear ordinary differential equation for which no exact solution exists. Therefore, a numerical solution method was required, which had to be incorporated into the Newton-Raphson iteration scheme in the Newmark Method.

Initially, a fourth-order Runge-Kutta algorithm (Burden and Faires 1985) was developed and tested against the exact solution for hysteretic displacement $z$ as a function of displacement $u$ that exists for the non-degrading, non-pinching BWBN hysteresis model. Without the pinching and stiffness degradation terms in the model, the equation becomes a linear ordinary differential equation that has a piece-wise continuous exact solution defined by four equations that depend on the sign of velocity and displacement. Testing indicated that the numerical solution provided a very good approximation of the exact solution. Figure 3.2 shows a typical non-pinching, non-degrading hysteresis loop, with the associated exact equations, for one cycle of a sinusoidal input displacement. 

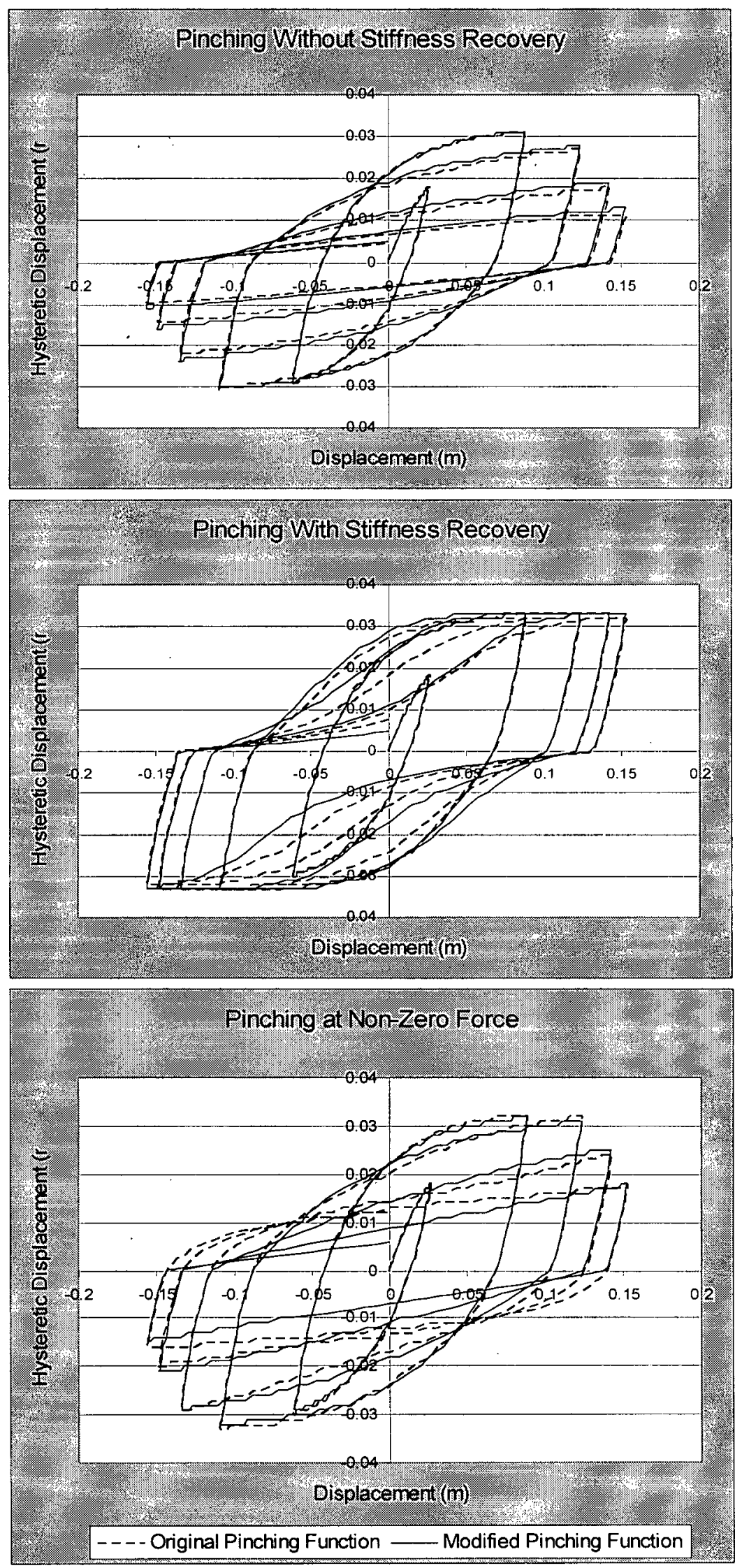

Figure 3.1: BWBN Model Pinching Function Comparison 


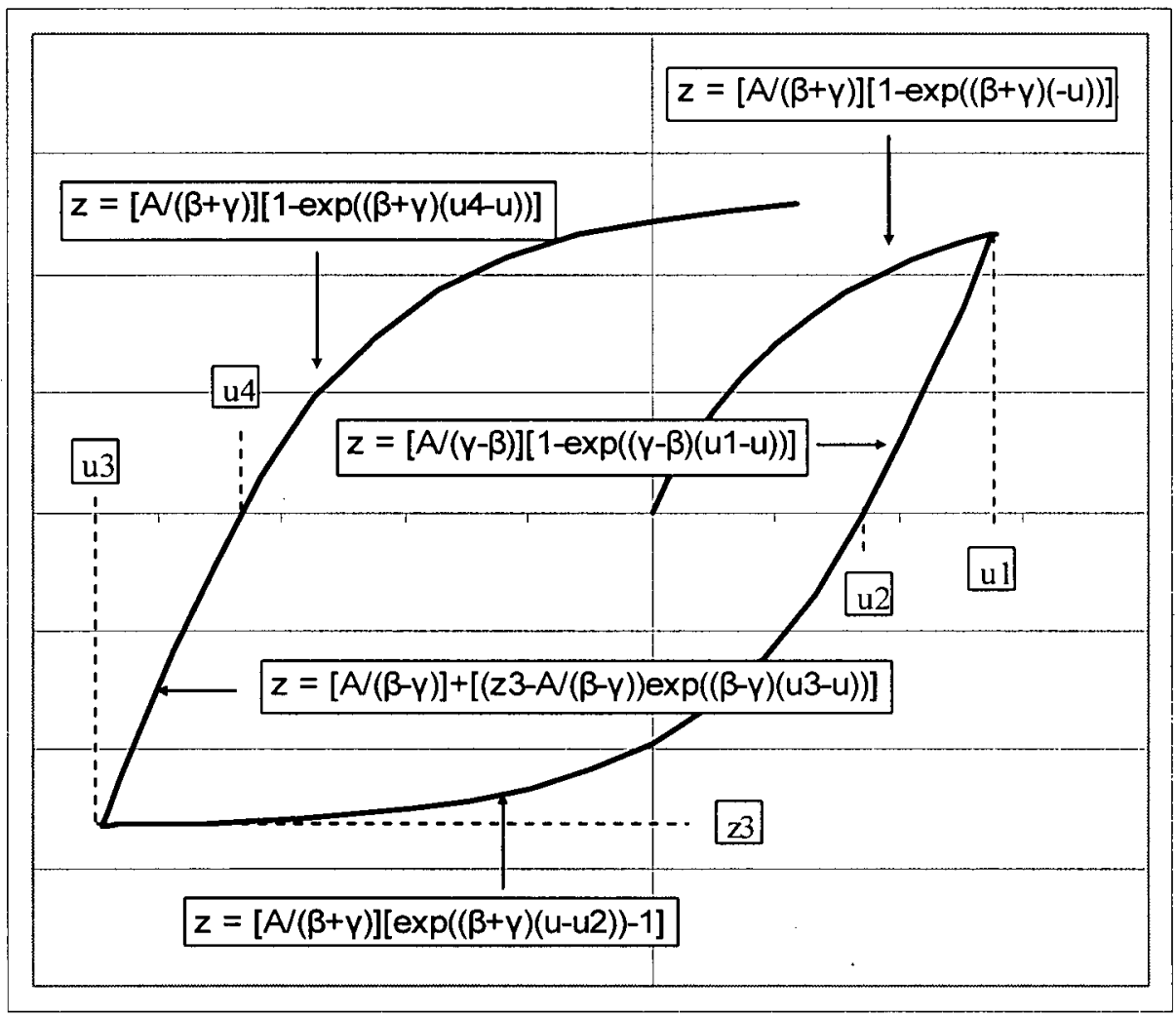

Figure 3.2: Exact Solution of Non-Pinching, Non-Degrading BWBN Model

The Runge-Kutta scheme is not usually the most computationally efficient method for solving non-linear ordinary differential equations but it was the starting point for developing a solution method because it succeeds in virtually all applications. The RK scheme is a one-step method where the approximation for $z_{i+1}$ involves information from the previous step $z_{i}$ only, solution methods that use information from several previous steps are termed multi-step predictor-corrector methods. Predictor-corrector methods are more computationally efficient than Runge-Kutta for many smooth systems but they are more difficult to start-up because of the need for more past information. A predictor-corrector solution scheme called the Adams Fourth-Order PredictorCorrector Method (Burden and Faires 1985) was tested against the Runge-Kutta scheme and found to give the same accuracy $(\sim 0.01 \%-0.02 \%$ difference) for the final value of $z$ for the same number of sub-steps $i$ within each time-step of a cyclic motion record. The 
Adams Method has the added advantage that it runs approximately $25 \%$ faster. In this method, the fourth-order Runge-Kutta scheme is used to obtain starting values for the four-step Adams-Bashforth predictor method, which is then corrected by one iteration of the three-step Adams-Moulton method. The number of predictor-corrector steps following start-up depends on the level of accuracy required of the numerical solution.

Implementation of the Adams Fourth-Order Method within the Newton-Raphson iterative process of the Newmark Method was initially completed using 100 predictorcorrector steps within each Newton-Raphson iteration. This number of steps was selected as a good balance between accuracy and run-time following a comparison of numerical hysteretic displacement results with the exact solution for a non-pinching, non-degrading system undergoing cyclic oscillation. Displacement error varied from $0.04 \%$ using 1000 predictor-corrector steps to $1.43 \%$ using five steps. The error for 100 steps was $0.32 \%$.

Following testing of the completed Newmark algorithm using real earthquake time-histories as the input excitation, it was determined that response analysis time could be improved significantly if the number of steps in the Adams Method solution algorithm could be reduced, while maintaining accuracy. Therefore, a new Adams Method algorithm was developed that uses adaptive step sizes for solving the ODE describing the hysteresis loop. Step size is adjusted according to the error estimate determined by comparing the predicted and corrected values of the dependent variable (i.e. $z$ ) after each cycle of the predictor-corrector process. Step size is iteratively reduced by a factor of one-half until the error is within the prescribed tolerance and iteratively increased by a factor of two when the error is less than $1 \%$ of the prescribed tolerance.

\subsubsection{Consideration of Degrading Natural Frequency}

Degradation in stiffness as the structural system response reaches the inelastic range has the effect of increasing the natural period of the structure, which affects the way the structure responds to subsequent excitation. The feasibility of modeling the 
degradation of natural frequency during non-linear response was assessed by calculating the dynamic response of systems in which the original, undamaged natural frequency was multiplied by the square-root of the stiffness ratio at the beginning of each time-step. The stiffness ratio was defined as the ratio of current hysteretic stiffness to original hysteretic stiffness. Initial attempts to update the natural frequency after each iteration of the Newton-Raphson procedure resulted in convergence problems and instability in the response calculation.

Assessment of the degrading natural frequency model showed that a minimum allowable stiffness ratio was required to prevent convergence problems associated with large displacements during time-steps where hysteretic stiffness approached zero. This situation, which may arise at response velocity sign changes or in the pinched region of the hysteresis loop, causes the stiffness ratio to approach zero and, consequently, natural frequency goes to zero. In this event, post-yield stiffness, given by the third term in Eq. [4], also approaches zero and extremely large deflections are incurred resulting in model convergence problems and instability.

Limiting the reduction in natural frequency by adding a minimum allowable stiffness ratio to the dynamic analysis algorithm tended to eliminate the convergence problem but added an unwanted additional parameter to the hysteresis model. In fact, from a system identification perspective, updating the natural frequency throughout a non-linear dynamic analysis effectively adds two parameters to the hysteresis model; the minimum stiffness ratio and an implicit parameter that is similar to the stiffness degradation parameter $\left(\delta_{\eta}\right)$. Consequently, the system identification process for the degrading natural frequency model will identify values of; $\alpha, \beta, \gamma, \delta_{v}, \delta_{\eta}, \varphi, \mu$ and $v$ that differ from those that would be identified without consideration of natural frequency degradation. However, the end result is the same, a phenomenological hysteresis model that provides a close match between model response and an input real response record or input pseudo-static test data. This implies that there is no need to include natural frequency degradation in the dynamic analysis algorithm since inclusion only alters the 
hysteresis model parameters to match the given input at the cost of an additional hysteresis parameter and possible convergence problems.

It should be noted that accounting for the inelastic response phenomena of natural period elongation within the phenomenological hysteresis model may increase the risk of model error when hysteresis parameters are estimated from pseudo-static test data. In a pseudo-static test there is little or no dynamic effect present in the response, therefore, the true impact of a degrading natural frequency is not reflected in the input data used for hysteresis parameter identification. This may in turn cause the pseudo-static model parameters to underestimate the true dynamic displacement response which now includes the actual consequence of a degrading natural frequency. This risk of underestimating the response displacement is not really the fault of accounting for period elongation within the hysteresis model, nor the fault of the system identification procedure itself, which only seeks to duplicate the input data, rather it is an unavoidable consequence of using pseudo-static data to calibrate a dynamic model.

\subsubsection{Parameter Identification}

As stated in Sec. 3.3.4.1, the determination of appropriate values for the modified hysteresis model parameters; $\alpha, \beta, \gamma, \delta_{\nu}, \delta_{\eta}, \varphi, \mu$ and $v$ contained in Eqs. [4, 5, 8, 9] is known as a system identification problem, or more accurately, as a parameter estimation problem. System identification is the general name given to a wide field that seeks to infer a mathematical or algorithmic model of a dynamic system based on observed data from the system. Parameter estimation is the second stage of the two-stage system identification process that determines the numerical values of all the parameterized elements of the mathematical model identified in the first stage. In the present application, the model from the first stage of the system identification process is the newly modified version of the BWBN hysteresis model, which may be called a gray-box within the overall system framework governed by the equation of motion. The term gray-box is used to describe a model in which the adjustable parameters have a physical interpretation such as the stiffness degradation parameter in the BWBN model. A model 
in which the parameters are simply vehicles for adjusting the fit to the available data and do not reflect physical considerations in the system is referred to as a black-box.

There are two common approaches to minimizing the error between a predictive model and the observed data on which it is based (Ljung 1999). The first approach, termed the prediction-error approach, is to form a scalar-valued norm or criterion function that measures the size of the prediction error and then choose the parameters that minimize the norm or function. This approach contains several well-known procedures such as the Least Squares Method and the Maximum Likelihood Method. The other approach, termed the correlation approach, is to require that the prediction error be uncorrelated with a given data sequence. In other words, the model parameters must be chosen such that the prediction error at every step in the sequence of observed data is independent of the previous steps. This approach is the basis for various instrumentvariable methods, which are most suited to auto-regression type (ARX) system models that describe the relationship between input and output using a linear difference equation containing previous values of the output variable. Using this type of model is equivalent to treating the entire system as a black-box, which by definition casts the parameter identification problem completely in the observation space, in terms of the observed input and output, without any reference to the underlying mechanics of the system.

Given that the parameters to be identified apply only to the gray-box hysteresis model and not an overall black-box system, the prediction-error approach was adopted for developing an algorithm to identify hysteresis parameters from input data. For reasons of simplicity, the quadratic norm of the Least Squares Method was chosen over the likelihood function of the Maximum Likelihood Method to measure the size of the prediction error associated with a given trial parameter vector $\theta$. Therefore, the generalized error norm is given by:

$$
V_{N}\left(\theta, Z^{N}\right)=\frac{1}{N} \sum_{t=1}^{N} l\left(\varepsilon_{F}(t, \theta)\right)
$$


where l(.) is the quadratic norm, $l(\varepsilon)=1 / 2 \varepsilon^{2}, Z^{N}$ is the observed dataset and the prediction error sequence $\varepsilon$ is given by:

$$
\varepsilon(t, \theta)=y(t)-\hat{y}(t \mid \theta)
$$

For predictor models that are a linear function of the parameters to be identified, the prediction error sequence becomes:

$$
\varepsilon(t, \theta)=y(t)-\varphi^{T}(t) \cdot \theta
$$

and the resulting least-squares norm, given by substitution into Eq. [10], becomes a quadratic function in $\theta$ that can be minimized analytically to determine the optimal parameter set. In general, however, Eq. [10] cannot be minimized analytically, as is the case for the gray-box hysteresis model, therefore, the minimum has to be found by iterative numerical techniques.

Numerical minimization methods can be divided into three groups; (i) methods using values of the function $V_{N}$ only, (ii) methods using values of the function $V_{N}$ and its gradient, and (iii) methods using values of the function $V_{N}$, its gradient and its Hessian, which is the second derivative matrix or curvature matrix. For the special case of the Least Squares Method, the gradient of the criterion function is given by:

$$
V_{N}^{\prime}\left(\theta, Z^{N}\right)=-\frac{1}{N} \sum_{t=1}^{N} \psi(t, \theta) \cdot \varepsilon(t, \theta)
$$

where $\psi(t, \theta)$ is the $d x p$ gradient matrix of $\hat{y}(t \mid \theta)$ with respect to $\theta$. This leads to a family of search routines given by:

$$
\hat{\theta}_{N}^{(i+1)}=\hat{\theta}_{N}^{(i)}-\mu_{N}^{(i)}\left[R_{N}^{(i)}\right]^{-1} V_{N}^{\prime}\left(\hat{\theta}_{N}^{(i)}, Z^{N}\right)
$$


where $R_{N}{ }^{(i)}$ is a $d x d$ matrix that modifies the search direction and $\mu_{N}{ }^{(i)}$ is the step size. If $R_{N}{ }^{(i)}$ is defined as the identity matrix, Eq. [14] becomes the steepest-descent method, which corresponds to the second group of methods listed previously. This method has the drawback that it is fairly inefficient close to the minimum of Eq. [10], which is the reason the Hessian is used in the third group of methods, to improve efficiency. The Hessian for the Least Squares Method is given by:

$$
V_{N}^{\prime \prime}\left(\theta, Z^{N}\right)=\frac{1}{N} \sum_{t=1}^{N} \psi(t, \theta) \cdot \psi^{T}(t, \theta)-\frac{1}{N} \sum_{t=1}^{N} \psi^{\prime}(t, \theta) \cdot \varepsilon(t, \theta)
$$

where $\psi^{\prime}(t, \theta)$ is the $d x d$ Hessian of $\varepsilon(t, \theta)$. The third group of methods utilizes the Hessian as the search direction matrix $R$, which then makes Eq. [14] a Newton method. It is quite costly, however, to compute all the terms of $\psi^{\prime}$ so the second term in Eq. [15] is typically ignored, which is equivalent to replacing the Newton-Raphson method with the modified Newton-Raphson method. This is permissible since a good estimate of the Hessian is only required in the vicinity of the minimum for a Newton method and the term multiplying the second derivative in Eq. [15] is the random error $\varepsilon(t, \theta)$, which can have either sign and should in general be uncorrelated with the model. Therefore, the second derivative terms tend to cancel out when summed from $t=1 \ldots N$. The actual implementation of a search scheme using the Hessian is commonly done using a method called the Levenberg-Marquardt procedure that smoothly varies between the two extremes of Eq. [14], the steepest descent method and the inverse-Hessian method. In this procedure, the step-size is set equal to unity $\left(\mu_{N}^{(i)}=1\right)$ and the search direction matrix, $R$, is modified by adding a parameter to the Hessian as follows:

$$
R_{N}^{(i)}(\lambda)=\frac{1}{N} \sum_{i=1}^{N} \psi\left(t, \hat{\theta}_{N}^{(i)}\right) \cdot \psi^{T}\left(t, \hat{\theta}_{N}^{(i)}\right)+\lambda I
$$

where $\lambda$ is a positive scalar that is used to control the convergence in the iterative scheme. In a search region far from the minimum value of the norm, $\lambda$ is set to a large value, which effectively reduces the step-size and turns the search direction towards the 
gradient as in the steepest-descent method. As the minimum is approached, $\lambda$ is iteratively reduced to zero, which smoothly switches the procedure to the inverseHessian method.

While the notion of using a parameter estimation method that identifies the optimal model parameter vector $\theta$ through an automated search for the minimum of the least-squares norm is appealing, there is a practical difficulty in implementing such a scheme. To use the formulas given previously, the gradient of the prediction, $\psi(t, \theta)$, must be calculated at each step of the input data sequence. There are a number of methods for doing so, the choice of which depends on the model structure, but for each method the computational effort required to compute the gradient can be significant. The only alternative to expending that computational effort is to implement a Group 1 method, which uses values of the function $V_{N}$ only, in conjunction with a specific search pattern. Since the gray-box hysteresis model contains eight parameters to be estimated, resulting in an eight-dimensional gradient for a Group 2 or Group 3 method, the Group 1 method, combined with a directed search, was used to develop an algorithm for estimating the hysteresis model parameters.

It is an inherent feature of iterative search routines that only convergence to a local solution is guaranteed, in this case the local minimum of $V_{N}\left(\theta, Z^{N}\right)$. To find the global minimum, which gives the optimal parameter vector $\theta$, there is no other way than to start the iterative minimization routine at different feasible initial values and compare the results. For a physically parameterized model, such as the gray-box hysteresis model, feasible initial values and an associated search pattern may be determined from physical insight into the model structure. Consideration of the physical meaning of each of the modified BWBN hysteresis parameters showed that the dominant parameters are the post-yield stiffness parameter, $\alpha$, and the two parameters that control the nondegrading shape of the hysteresis loop, $\beta$ and $\gamma$. From that starting point, a three-stage algorithm was developed that begins by evaluating combinations of those three parameters that satisfy both Eq. [4], the differential equation of motion, and Eq. [5], the differential equation of hysteretic displacement, for a given input data record. The input 
data is taken from either a pseudo-static cyclic displacement test or an acceleration response time-history of the structure for which the hysteresis parameters are to be identified. The actual number of parameter combinations that satisfy both differential equations depends on the yield displacement of the structure but the upper limit on the possible number of combinations evaluated for least-squares error is set at 3,960,000. This covers values of $\alpha$ that range from 0.01 to 0.99 and values of $\beta$ and $\gamma$ that correspond to a yield displacement range of $0.007-2.000 \mathrm{~m}$.

Once the least-squares error combination of the three dominant parameters is roughly determined, the second stage of the algorithm evaluates trial values of the pinching parameters, $\varphi, \mu$ and $v$, while refining the precision of $\alpha, \beta$ and $\gamma$. As many as 69,300 parameter combinations are evaluated for least-squares error, depending on the initial values of $\alpha, \beta$ and $\gamma$ identified in the first stage. The decision to identify the pinching parameters before the stiffness degradation parameter, $\delta_{\eta}$, which overlap in their physical role in the hysteresis model, was made following comparisons of model fits with each parameter type identified first. Identifying pinching parameters before the stiffness degradation parameter is perhaps intuitive since the stiffness reduction associated with pinching is considerably larger, but localized to a portion of each loading phase, than the stiffness reduction associated with the stiffness degradation parameter, which applies throughout the hysteresis cycle. Therefore, attempting to fit the stiffness degradation parameter before the pinching parameters results in identifying a range of possible values with similar and relatively large least-squares errors that adequately model the stiffness reduction in a certain portion of the loading phase but do a poor job in the remainder of the loading phase.

The third stage of the parameter estimation algorithm finalizes the hysteresis model parameter vector by evaluating the least-squares error for 289 trial values of the strength and stiffness degradation parameters, $\delta_{\nu}$ and $\delta_{\eta}$, in combination with the other parameters identified in the second stage. 


\subsubsection{Parameter Adjustment for MDOF Structures}

For non-linear dynamic analysis of a multiple-degree-of-freedom structure, the hysteresis model parameters, which are identified from structural test data corresponding to a certain stiffness and yield strength, must be adjusted for each different storey stiffness and yield strength. This adjustment is straightforward for the parameters related to yield strength, $\gamma$ and $\beta$, which are simply modified by the ratios of storey stiffness to test structure stiffness and storey yield strength to test structure yield strength to maintain the proper storey yield displacement. The post-yield stiffness parameter, $\alpha$, is assumed to remain constant. The remaining hysteresis parameters control pinching behaviour and strength and stiffness degradation as a function of the dissipated energy, which is calculated as the area of the hysteresis loop. The assumption that is made in adjusting these parameters is that a similar structural system with a different stiffness and/or yield strength that experiences a similar level of energy dissipation, normalized with respect to the calculated yield energy, will exhibit a similar degree of pinching and strength and stiffness degradation as the response history progresses. Using this assumption, the energy-related hysteresis parameters for each storey are adjusted such that the product of the parameter and the constant terms multiplying the normalized dissipated energy is constant (see Eqs. [8] and [9]).

\subsubsection{Fourier Analysis and Power Spectrum Estimation}

Generation of artificial earthquakes for Monte Carlo analysis of structural dynamic response requires a frequency spectrum source that contains the spectral characteristics of the type of earthquake being studied for its impact on structural response. This frequency spectrum source may be a white-noise Gaussian process that is filtered to give the required spectral shape or, alternatively, it may be determined from an actual earthquake record. When using an actual record the power spectral density, which describes the contribution of the individual harmonic components in a random signal, must be determined from a Fourier transform analysis of the discretely sampled ground acceleration data. This analysis is typically performed by the well-known fast Fourier 
transform or FFT algorithm. The salient details of the FFT algorithm and an associated spectral analysis algorithm that were adapted for use in the software application being developed in this project are summarized in the following.

\subsubsection{FFT Algorithm}

The FFT algorithm, first developed by Cooley and Tukey in 1965, is based on a recursive application of the Danielson-Lanczos Lemma, which was developed in 1942. The Danielson-Lanczos Lemma divides the discrete Fourier transform of a function consisting of $N$ discretely sampled points into the sum of two discrete Fourier transforms, each of length $N / 2$. Each individual transform is made up of the even-numbered and odd-numbered points in the original $N$, respectively. Through recursive application of the Danielson-Lanczos Lemma, the original Fourier transform is repeatedly subdivided in half until transforms of unit length are obtained, assuming the original $N$ is an integer power of two. If the length of the original dataset is not a power of two, it must be padded with zeroes up to the next power of two. With the input data subdivided down to transforms of unit length, the Fourier transform of the single data point is simply the identity operation, in other words, the transform of the point is the point itself. The computational efficiency of the FFT algorithm over the slow Fourier transform comes from a data indexing system that is based on recording the pattern of even and odd data points during recursive application of the Danielson-Lanczos Lemma as a binary number. Using a bit reversal procedure, the original data is rearranged into bit-reversed order that allows for a highly efficient method of recombining the one-point transforms into two-point transforms, the two-point transforms into four-point transforms and so on until the final transform is obtained.

The efficiency of the FFT algorithm reduces the computational effort required to estimate a Fourier transform of length $N$ from an $O\left(N^{2}\right)$ process using the slow Fourier transform algorithm to an $O\left(N \log _{2} N\right)$ process. This difference is best understood when illustrated by the example of a function with $N=10^{6}$ discrete points. The computational effort involved in using the slow algorithm is 50,000 times greater than that using the 
FFT, which in terms of computation time translates to one second versus 14 hours. For an earthquake acceleration record with $N=1500$ discrete points, the computational effort in estimating the Fourier transform is decreased by a factor of 142 when using the FFT algorithm.

It is worth noting that other classes of FFT algorithms exist, such as those that subdivide the initial dataset of length $N$ to some small power of two, rather than to the trivial transform of unit length. These are called base-4 FFT's or base-8 FFT'S. There are also FFT algorithms for datasets of length $N$ that are not a power of two. They work by using relations analogous to the Danielson-Lanczos Lemma that subdivide the initial problem into successively smaller problems, not by factors of two, but by whatever small prime factors happen to divide $N$. The larger the largest prime factor of $N$ is, the slower this method becomes until it reverts to the slow Fourier transform when $N$ is prime and no subdivision is possible. One example of this method is the Winograd Fourier transform class of algorithms that, for some values of $N$, may be up to twice as fast as the simpler FFT algorithms based on dataset lengths of an integer power of two. This advantage in speed, however, is offset by considerably more complicated data indexing and the fact that the operation cannot be done in-place, where the original dataset is replaced by its Fourier transform.

\subsubsection{Power Spectrum Algorithm}

Following computation of the Fourier transform of a function or dataset, the estimation of the associated power spectral density (PSD) depends on the type of normalization applied to one of several possible descriptions of the functions total power. For a function $c(t)$ sampled at $N$ points to produce values $c_{0}, c_{1} \ldots c_{N-1}$, total power may be described by:

$$
\sum_{j=0}^{N-1}\left|c_{j}\right|^{2}
$$




$$
\begin{aligned}
& \frac{1}{T} \int_{0}^{T}|c(t)|^{2} d t \approx \frac{1}{N} \sum_{j=0}^{N-1}\left|c_{j}\right|^{2} \\
& \int_{0}^{T}|c(t)|^{2} d t \approx \Delta \sum_{j=0}^{N-1}\left|c_{j}\right|^{2}
\end{aligned}
$$

These descriptions of total power are termed the sum squared amplitude, mean squared amplitude and time-integral squared amplitude, respectively. If the estimation of the power spectral density of $c(t)$ is done using a periodogram estimator of the power spectrum, then the estimate is defined at $N / 2+1$ frequencies as:

$$
\begin{aligned}
& P(0)=P\left(f_{0}\right)=\frac{1}{N^{2}}\left|C_{0}\right|^{2} \\
& P\left(f_{k}\right)=\frac{1}{N^{2}}\left[\left|C_{k}\right|^{2}+\left|C_{N-k}\right|^{2}\right] \quad(k=1,2 \ldots N / 2-1) \\
& P\left(f_{c}\right)=P\left(f_{N / 2}\right)=\frac{1}{N^{2}}\left|C_{N / 2}\right|^{2}
\end{aligned}
$$

where $C_{k}$ is the discrete Fourier transform of the function $c(t)$ given by:

$$
C_{k}=\sum_{j=0}^{N-1} c_{j} e^{2 \pi i j k / N} \quad(k=0,1 \ldots N-1)
$$

and $f_{k}$ is defined only for the zero and positive frequencies as follows:

$$
f_{k}=\frac{k}{N \Delta}=2 f_{c} \frac{k}{N} \quad(k=0,1 \ldots N / 2)
$$

The normalization associated with the periodogram estimate of the power spectral density is such that the sum of the $N / 2+1$ values of $P$ is equal to the mean squared amplitude of the function $c(t)$. This can be seen by comparing Eq. [18] with Parseval's theorem, which is given by: 


$$
\sum_{k=0}^{N-1}\left|h_{k}\right|^{2}=\frac{1}{N} \sum_{n=0}^{N-1}\left|H_{n}\right|^{2}
$$

As seen from Eqs. [20], the PSD is defined over the frequency range from zero to the Nyquist critical frequency, $f_{c}=1 /(2 \Delta)$ where $\Delta$ is the sampling time interval. Integration of the PSD beyond the Nyquist frequency is unnecessary since, according to the sampling theorem, any power outside of the Nyquist interval will be aliased into the interval by the act of discrete sampling. In other words, the frequency components of a function that is not bandwidth limited to less than the Nyquist frequency are aliased, or falsely translated, into the interval $\left(-f_{c}, f_{c}\right)$ automatically by discrete sampling. This is a result of the fact that estimation of the Fourier spectrum from the sampled data, which is taken from a continuous analogue signal, results in an estimated spectrum that is periodic with period $1 / \Delta$ and symmetric about the zero frequency position. Any frequencies higher than $f_{c}$ in the original signal distort the calculated spectrum by aliasing towards higher frequencies, which distorts the Fourier coefficients for frequencies below the Nyquist frequency. However, if a continuous function $h(t)$ that is sampled at an interval $\Delta$ happens to be bandwidth limited to frequencies smaller in magnitude than $f_{c}$, then the function $h(t)$ is completely determined by its samples, $h_{n}$. In fact, $h(t)$ is given explicitly by the formula:

$$
h(t)=\Delta \sum_{n=-\infty}^{\infty} h_{n} \frac{\sin \left[2 \pi f_{c}(t-n \Delta)\right]}{\pi(t-n \Delta)}
$$

The spectrum distortion associated with aliasing can only be dealt with prior to sampling, once the signal has been discretely sampled there is little that can be done to remove aliased power. Therefore, to overcome aliasing, provision must be made ahead of time to sample the signal at a rate that gives at least two points per cycle of the highest frequency present, which is either known ahead of time from the natural bandwidth limit of the signal or else enforced by analog filtering of the continuous signal with a lowpass linear filter prior to sampling. Following data collection it is a simple matter to determine 
if the signal has been properly sampled, if the Fourier transform does not approach zero as the frequency approaches $f_{c}$ from below then it is likely that frequency components outside the Nyquist range have been translated into the critical range. In this event, the Fourier transform cannot be assumed to be zero beyond the Nyquist frequency.

From the perspective of earthquake spectrum estimation and dynamic structural response analysis, the issue of aliasing is not particularly worrisome since a lightly damped structure, which is applicable to most practical civil engineering structures, acts like a narrow-band filter resulting in a sharply peaked response at the undamped natural frequencies of the structure. This means that the major contribution of the power spectral density to the dynamic response is obtained in the vicinity of the natural frequencies of the structure and the value of the power spectral density outside that vicinity does not significantly affect the response (see Eq. 2.11 and Fig. 2.1). Therefore, under the assumption that the earthquake ground acceleration has been sampled to capture frequencies up to perhaps $25 \mathrm{~Hz}$, the spectrum distortion associated with aliasing will have little if any effect on even the higher modes of response, which will typically have natural frequencies much less than $25 \mathrm{~Hz}$.

Of more concern in calculating the periodogram estimator of a power spectrum are the issues of accuracy in the face of frequency leakage and minimizing the variance of the estimator. The first issue of frequency leakage between the discrete frequency components in the periodogram is a consequence of the values of $P\left(f_{k}\right)$ in the periodogram estimate not being exactly equal to the continuous $P(f)$ at frequency $k$ since $f_{k}$ is supposed to be representative of a whole frequency bin extending from halfway from the preceding discrete frequency to halfway to the next one. This problem is addressed by data windowing in which the original data is multiplied by a window function $w_{j}$ that changes smoothly from zero to a maximum and then back to zero as $j$ ranges from 0 to $N$. A smooth window function merely improves on the square window function inherent in any finite sample length $N$ that has been obtained, in effect, by multiplying an infinite record by zero except during the total sampling time, $T=N \Delta$, when it is multiplied by 1 . Thus the square window is in essence an ideal lowpass filter with a stopband or cutoff 
frequency equal to $1 / T$. By the convolution theorem, the Fourier transform of the product of the data and the window function is equal to the convolution of the data's Fourier transform with the window's Fourier transform. Without data windowing, the Fourier transform of the inherent square window, which has large side lobes beyond the cutoff frequency due to the abrupt changes in window amplitude between zero and 1, results in a convolved Fourier transform with substantial components beyond the cutoff frequency. This in turn leads to leakage in the calculated power spectrum. Using a smooth window function counteracts frequency leakage by reducing the side lobes of the convolved Fourier transform (see Fig. 3.3). The window function that has been incorporated into the power spectrum algorithm of the software application is known as the Bartlett window, which has a triangular form given by the following:

$$
w_{j}=1-\left|\frac{j-\frac{1}{2} N}{\frac{1}{2} N}\right|
$$
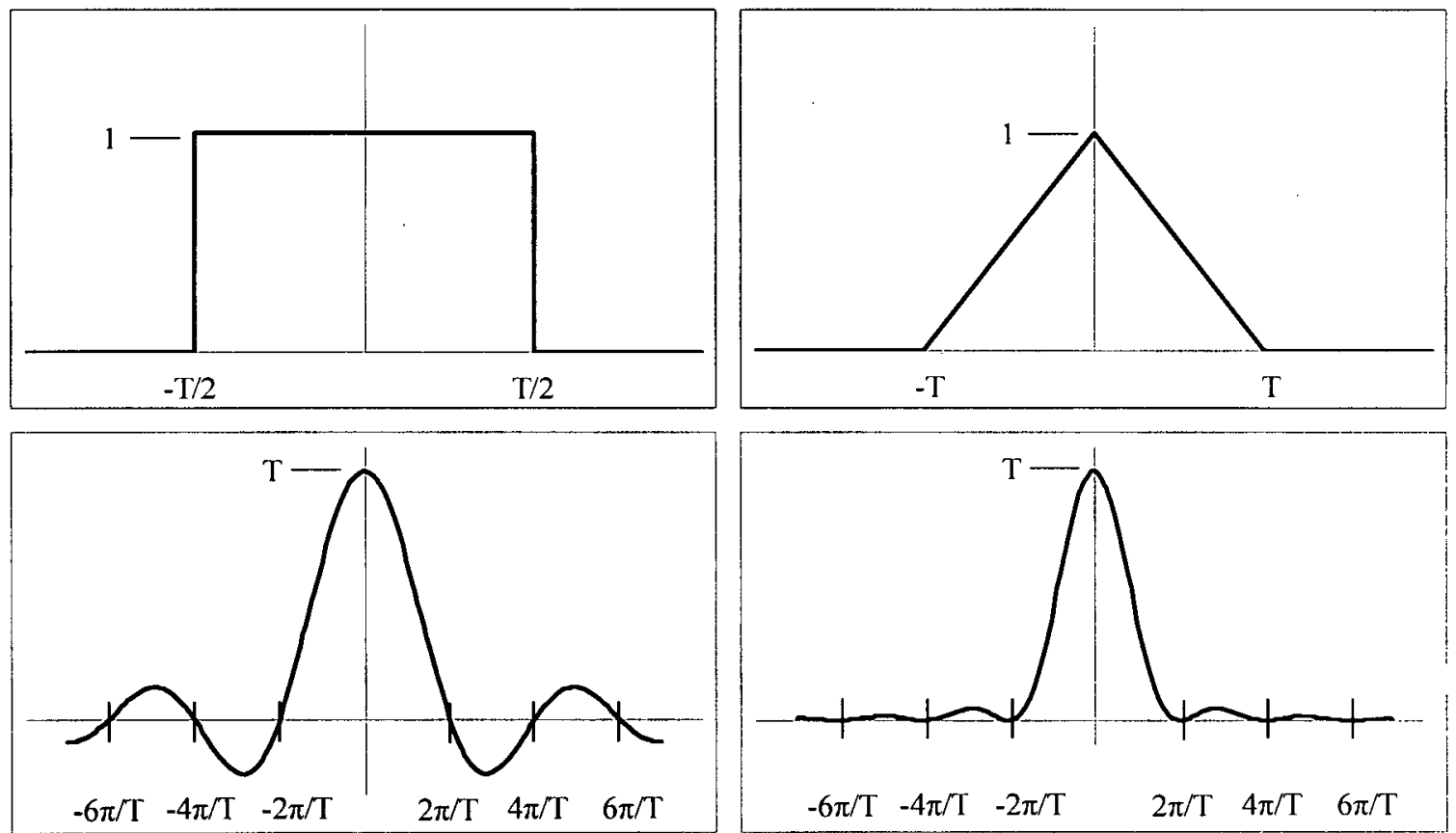

Figure 3.3: Square Window, Bartlett Window and Fourier Transforms 
The second issue in calculating the periodogram estimator of a power spectrum, which is minimizing the variance of the estimate, is achieved by a frequency averaging technique that segments the data into $K$ segments of $2 M$ data points that are separately FFT'd to produce $K$ individual periodogram estimates. These $K$ periodograms are then averaged to obtain a PSD estimate at $M+1$ frequencies between zero and the Nyquist frequency $\left(f_{c}\right)$. This averaging process reduces the variance of the PSD estimate by a factor that depends on how the data in each segment is overlapped with adjacent segments. The best method of overlapping depends on whether variance reduction is being done to obtain the smallest spectral variance from a fixed amount of computation or whether variance reduction is being done to obtain the smallest spectral variance per data point. In the first case it is best to segment the data without any overlapping, which requires $2 K M$ data points, and results in variance reduction by a factor $K$. In the second case, which minimizes variance from a fixed number of available sampled data points, the segments should be overlapped by one half of their length resulting in variance reduction by a factor of approximately $9 K / 11$ for $(K+1) M$ points.

Since the spectral analysis algorithm is intended to calculate the power spectrum of earthquake ground acceleration data that has already been recorded, the second method of variance reduction using overlapping segments of $2 M$ data points was the one that was incorporated into the calculation of the periodogram estimator. The value of $M$, which determines the number of frequency values between zero and $f_{c}$, is set within the algorithm based on the time-step of the input ground acceleration record. Table 3.1 lists the power spectrum frequency resolution for different input time-steps.

Table 3.1: Power Spectrum Frequency Resolution

\begin{tabular}{|c|c|}
\hline Time-Step (sec) & $M$ \\
\hline$\geq 0.04$ & 512 \\
\hline$\geq 0.02$ & 1024 \\
\hline$\geq 0.01$ & 2048 \\
\hline$<0.01$ & 4096 \\
\hline
\end{tabular}


The number of data segments, $K$, is determined from the frequency resolution, $M$, and the number of data points in the acceleration record, which is doubled in length prior to determining $K$. The acceleration record is then padded with zeroes to provide a total of $(K+1) M$ points for spectrum estimation.

\subsubsection{Acceleration Record Filtering}

An algorithm to frequency-filter the input earthquake record, as well as any generated earthquake records, was developed to allow for the removal of a chosen number of high frequency and low frequency components in the record(s). This filtering option was included in the software application to ensure that the ground acceleration record being used for structural dynamic response analysis is truly representative of a real earthquake. The ground displacement in a real earthquake, for example, typically oscillates around a zero mean and comes to rest with a small or zero final displacement. For certain records that contain low frequency components, however, the calculated ground displacement may oscillate around a dominant low frequency or exhibit significant drift over the duration of the earthquake, resulting in a final displacement that is seriously in error.

The filter that is used for removing unwanted frequencies from the acceleration record is a cosine-type window that transitions between 0 and 1 as follows:

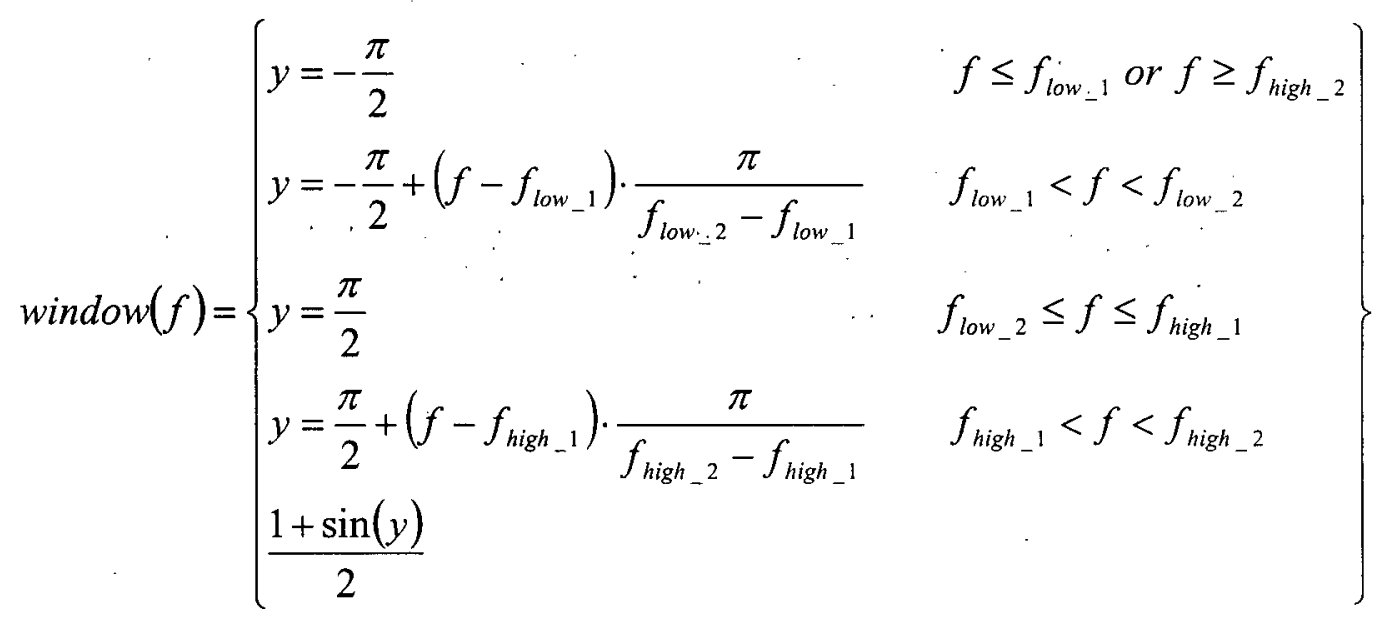


where $f_{\text {low } 1}, f_{\text {low_2 }}, f_{\text {high_1 }}$ and $f_{\text {high } 2}$ are the window transition frequencies. Using this window, the structure of the filtering algorithm is based on a visual inspection of the calculated ground displacement over the time-history of the acceleration record. The unfiltered ground displacement time-history is displayed onscreen in the software user interface and the user then adjusts the low frequency and high frequency window transitions until the resulting ground displacement time-history, which overlays the unfiltered time-history, is deemed acceptable. To aid in determining the effect of removing high and low frequency acceleration components; the minimum, maximum, average and final ground velocities and displacements are summarized in the visual display of the displacement time-history. This allows the user to, for example, adjust the cosine window transitions to filter the acceleration record such that the final ground velocity is close to the physically required value of zero. The calculation of the ground velocity and ground displacement time-histories from which the minimum, maximum, average and final values are determined is done in the frequency domain to reduce the accumulation of error inherent in the double integration procedure required in the time domain.

\subsubsection{Random Number Generation}

A reliable source of random numbers is essential for any sort of stochastic modeling or Monte Carlo analysis to ensure that a random quantity is as close to truly random as possible within the confines of a deterministic computer. An informal definition of randomness in the context of computer-generated sequences is that the deterministic algorithm that produces a random sequence should be different from, and in all measurable respects statistically uncorrelated with, the computer program that uses its output. The computational issues related to generating reliable random sequences are summarized in the following along with a description of the algorithms that were incorporated into the software application for use in randomizing structural properties and generating artificial earthquake time-histories. 
The simplest method of generating random numbers for use in a computer program is to use the routine that has likely been provided in the language the program is written in. While this is a convenient means of generating random numbers it can lead to serious violations of the assumption of uncorrelated random sequences when a large number of random numbers is required. System-supplied random number generators are typically linear congruential generators, which generate a sequence of integers $I_{1}, I_{2}, I_{3}$, ..., each between 0 and $m-1$, by the recurrence relation:

$$
I_{j+1}=a I_{j}+c(\bmod m)
$$

where $m$ is the modulus and $a$ and $c$ are positive integers called the multiplier and the increment, respectively. The recurrence given by Eq. [27] will eventually repeat itself with a period that has a maximum length $m$ for properly chosen values of $m, a$ and $c$. Improperly chosen values of the modulus, multiplier and increment will result in a considerably shorter period length. This then leads to two shortcomings of using a system-supplied random number generator, firstly, some computer manufacturers have made exceedingly poor choices for $m, a$ and $c$ (Press et al. 1999), thereby seriously shortening the repeat period. Second, the value of $m$, which is the largest possible random number, is often not very large on many computers. The American National Standards Institute (ANSI) standard for the C language, which was used to develop the software application, requires only that the system-supplied routine rand() return an integer that is at least 32,767 . This number is far too small for the random number intensive process of generating earthquake time-histories for Monte Carlo analysis. For example, generating a single Poisson process type earthquake similar to the 116 second Chile Llollelo event, sampled at $200 \mathrm{~Hz}$, requires approximately 70,000 random numbers, which means that the entire random number table would be used more than twice for each artificial record that was generated. Clearly, the records are then highly correlated and not close to being truly random as required in a Monte Carlo analysis that may use thousands of earthquake records. 
To eliminate the potential problems associated with system-supplied random number generators and machine specific choices for the recurrence equation parameters, a number of portable random number generators that can be implemented in various programming languages on various machines have been developed. The generator that was implemented in the software application is based on a multiplicative congruential algorithm given by:

$$
I_{j+1}=a I_{j} \quad(\bmod m)
$$

Using this simple recurrence relation, Park and Miller (1988) developed a Minimal Standard generator that is based on the choices:

$$
a=7^{5}=16807 \quad m=2^{31}-1=2147483647
$$

This Minimal Standard generator, first proposed in 1969 , has a period of $2^{31}-2 \approx 2.1 \mathrm{x}$ $10^{9}$ and has passed all theoretical statistical tests since its inception. The portability of this generator to essentially any programming language on essentially any machine is due to its ability to work with numbers generated by Eqs. $[28,29]$ that exceed the maximum value for a 32-bit integer, which is the limit for a high-level language. Using a 64-bit product register in Assembly language would allow the equations to be used directly but the implementation would not be portable between machines.

The implementation of the Park and Miller Minimal Standard random number generator in the software application returns a uniform random deviate between 0.0 and 1.0, exclusive of the endpoint values. A small improvement on the basic Minimal Standard algorithm, called the Bays-Durham shuffle, is included in the generator to remove subtle low-order serial correlations present in the basic generator. This shuffling algorithm shuffles the output such that the $j$ th value in the sequence, $I_{j}$, is output not on the $j$ th call, but rather on a randomized later call, which occurs at $j+32$ on average. This generator has been shown to pass all statistical tests up to the point where the number of calls starts to become on the order of $5 \%$ of the period $m$. Therefore, for applications that 
require less than approximately $100,000,000$ random numbers in a single calculation this generator has no known flaw. Clearly, this is well within the maximum demand imposed by the sort of Monte Carlo analysis mentioned previously. As an aside, there are other well-accepted random number generators with much longer periods that could have been incorporated into the software application, however, their relative execution times are significantly longer. These generators, which have periods of $\approx 2.3 \times 10^{18}$ and beyond, provide random number sequences that are, for all practical purposes, impossible to repeat on existing computers. These types of random number generators are used in cryptographic systems.

Since the randomization of structural properties requires random deviates that follow distributions other than the uniform distribution, provision was made to generate random numbers that also follow the normal, lognormal, Gumbel, Frechet and Weibull distributions. Random sequences with each of these distributions are generated from a sequence of random numbers uniformly distributed between 0.0 and 1.0 using the wellknown inverse transformation method. This method uses the inverse of the cumulative distribution function (CDF) of a variable to map the uniform random numbers to values that follow the required distribution. 


\section{CHAPTER 4}

\section{SOFTWARE FRAMEWORK AND USER-INTERFACE}

\subsection{INTRODUCTION}

Following development of the key solution algorithms and numerical components that form the foundation of PSResponse, which was outlined in Chapter 3, the overall architecture of the software that links all the sub-components together with the user interface was constructed. Chapter 4 summarizes that architecture beginning with a flow-chart description of the core computational framework in Section 4.2. In Section 4.3 , the structure of the user-interface that overlays the computational framework is briefly described followed by a more detailed look at the user-interface in Section 4.4. In this more detailed look, the key software features available to the user are illustrated using screen captures taken from various types of dynamic analysis.

\subsection{COMPUTATIONAL FRAMEWORK}

The calculating engine of PSResponse consists of approximately 79 algorithms linked together in an object-oriented framework that can be loosely divided into six groupings for illustrative purposes. These six groupings are shown in Figure 4.1 to Figure 4.6. With the exception of the User Input box in Figure 4.1 and the Store Results box in Figure 4.5, each box in each figure corresponds to a separate algorithm that is linked or called by other algorithms in the manner shown. For purposes of simplicity and clarity, some algorithms are shown in more than one figure to avoid too many cross-links between figures.

It should be clear that the computational framework illustrated in Figure 4.1 to Figure 4.6 shows all the algorithms and associated links to provide the entire 
functionality of PSResponse. The actual logical path that a particular PSResponse analysis would follow depends, of course, on the options selected and type of dynamic analysis performed.

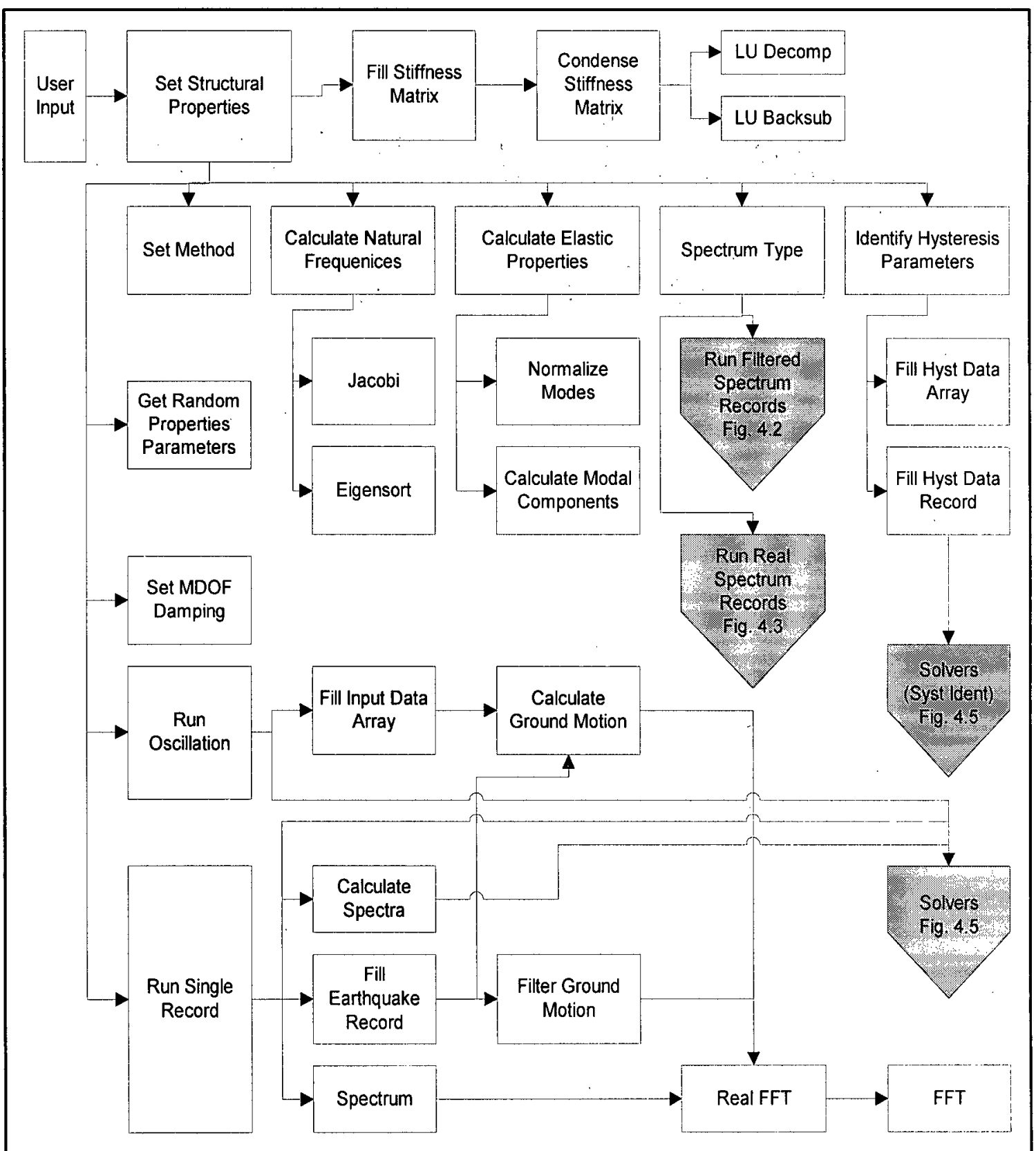

Figure 4.1: Computational Framework - Initialization 


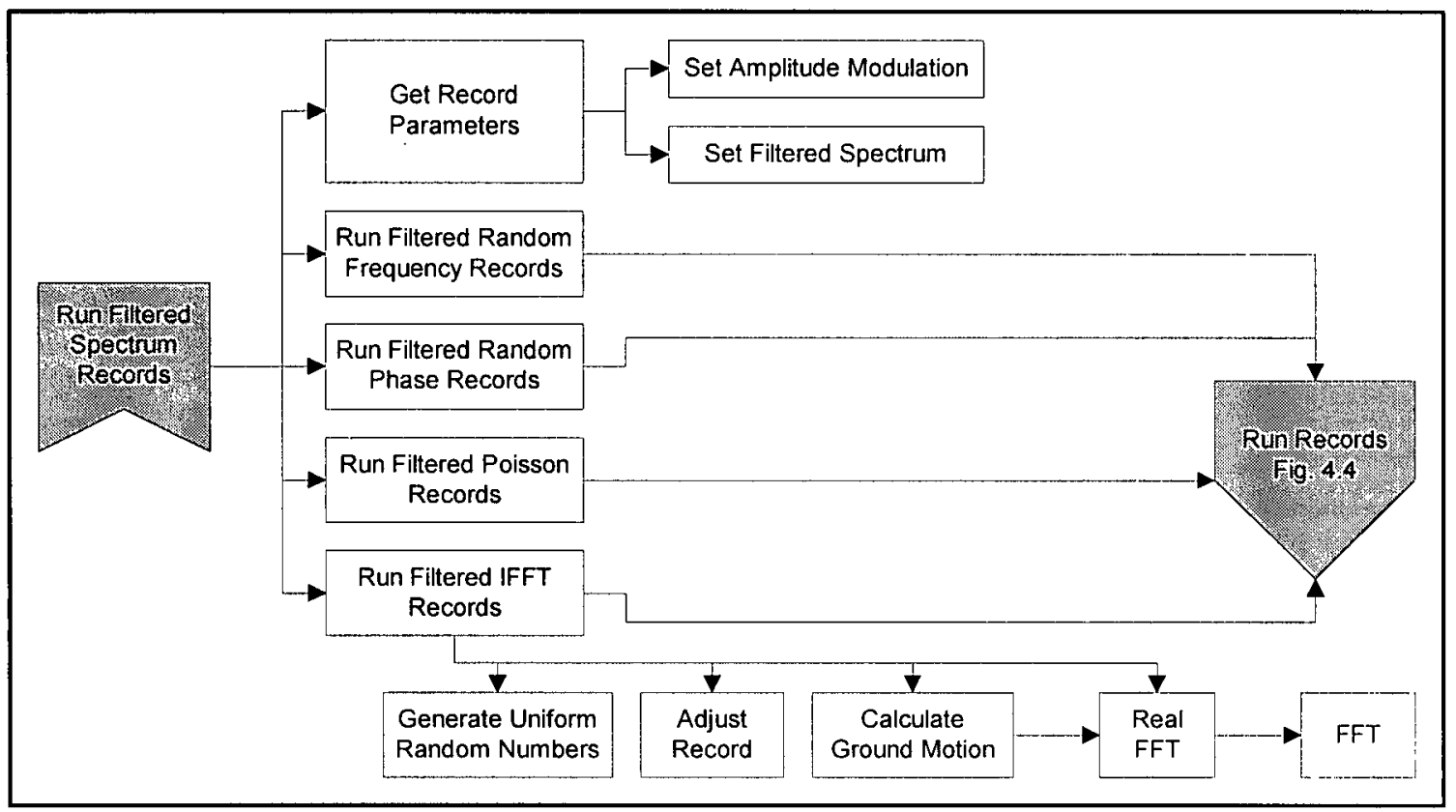

Figure 4.2: Computational Framework - Multiple Record Analysis (Filtered Spectrum)

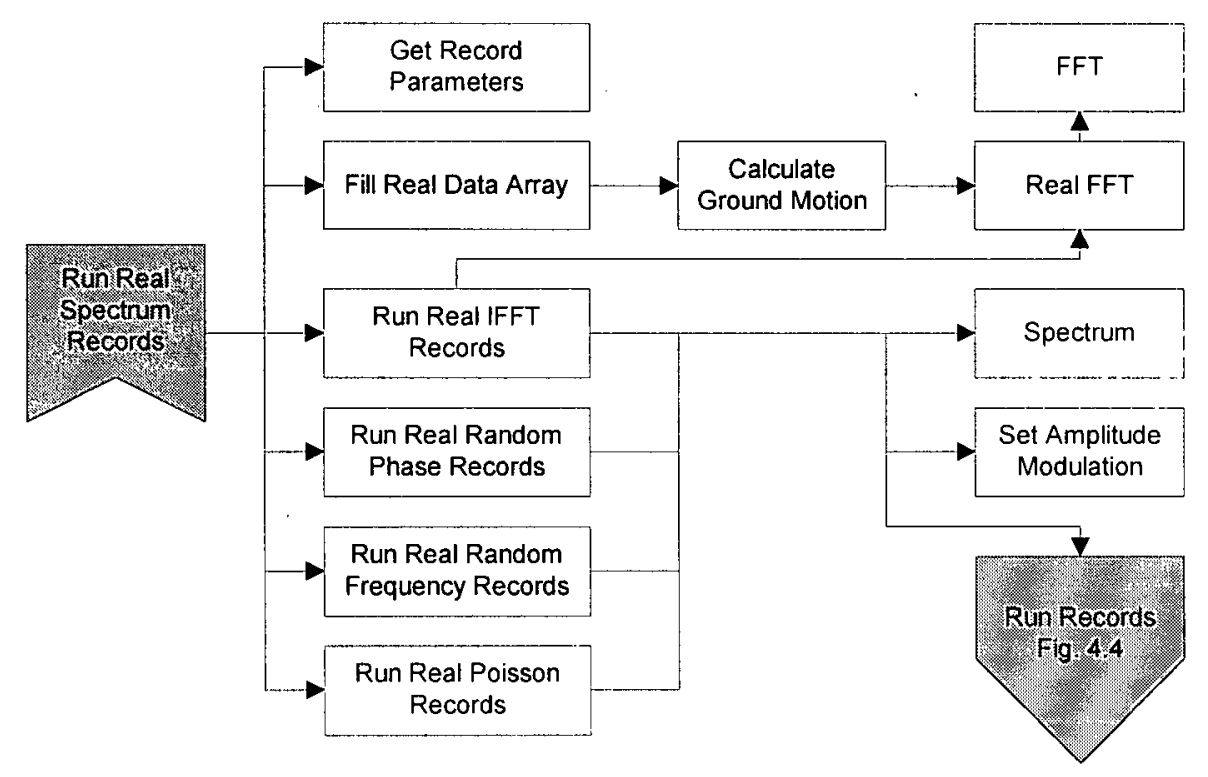

Figure 4.3: Computational Framework - Multiple Record Analysis (Real Spectrum) 


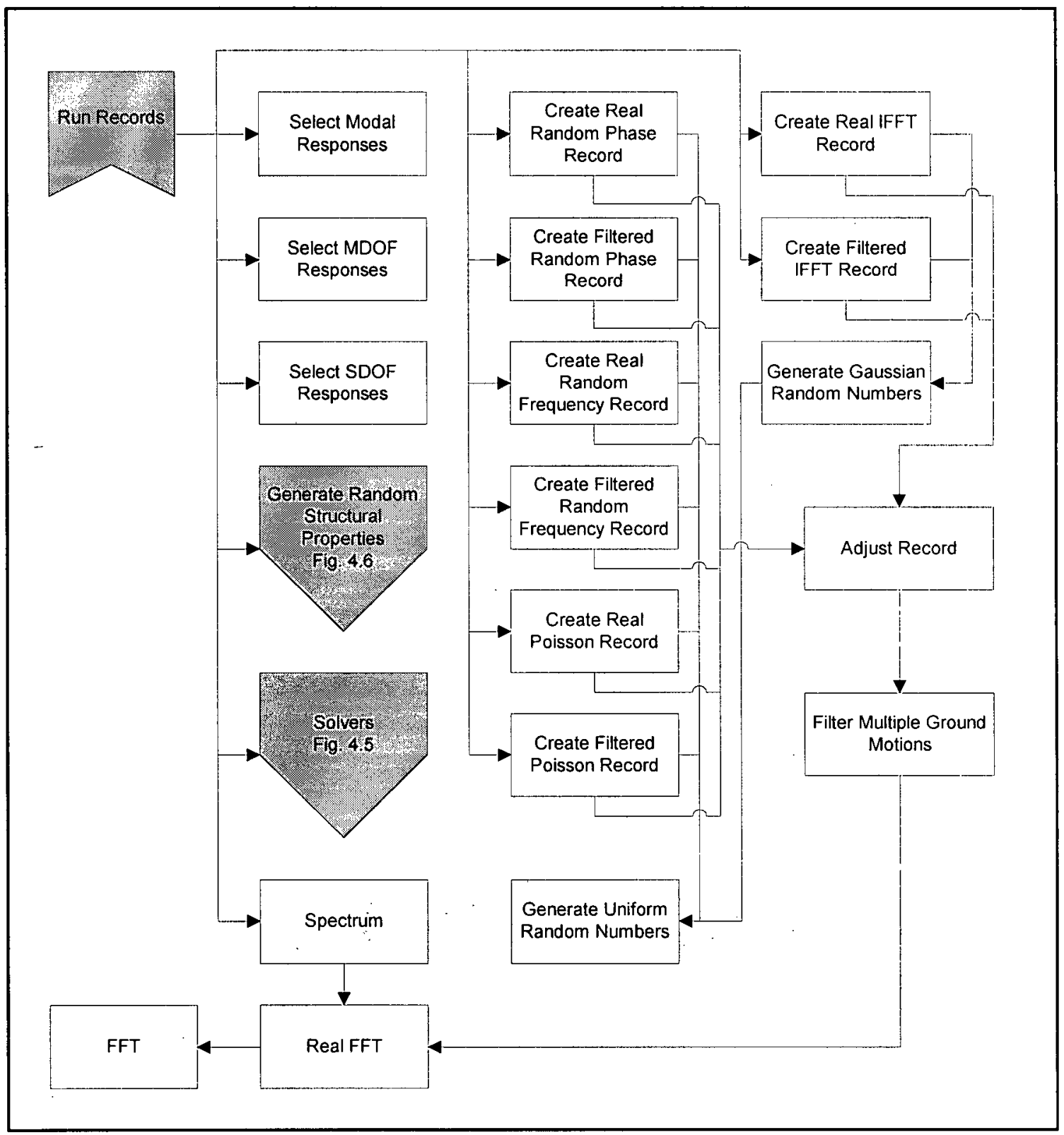

Figure 4.4: Computational Framework - Run Multiple Records

\subsection{USER-INTERFACE STRUCTURE}

The user-interface that overlays the computational framework is based on a wizard manager architecture that guides the user through a series of Windows-driven input and output dialog boxes. The wizard manager algorithm, which acts as the link 
between the dialog boxes and the computational framework, determines the dialog box sequence, passes information between dialog boxes, passes input data to computational algorithms and stores both input data and output arrays. The basic structure of the algorithm, in terms of the general order in which user input is collected, is outlined by the simplified flow-chart in Figure 4.7. Although not shown, there are a total of 36 separate paths through the flow-chart in which the flow-chart boxes typically represent a single dialog box in the user-interface. In some instances, however, several dialog boxes are associated with a single flow-chart box.

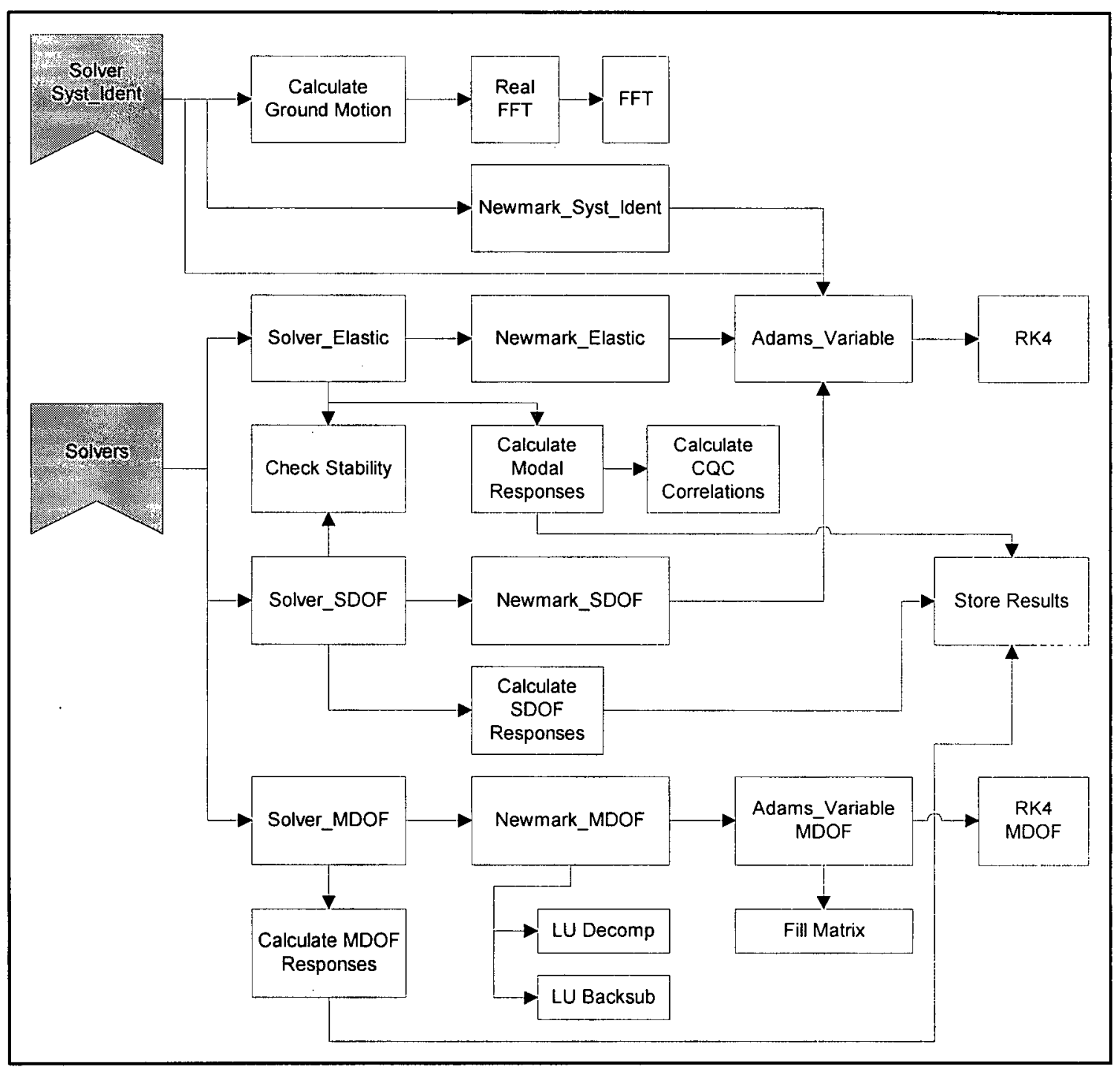

Figure 4.5: Computational Framework - Solvers 


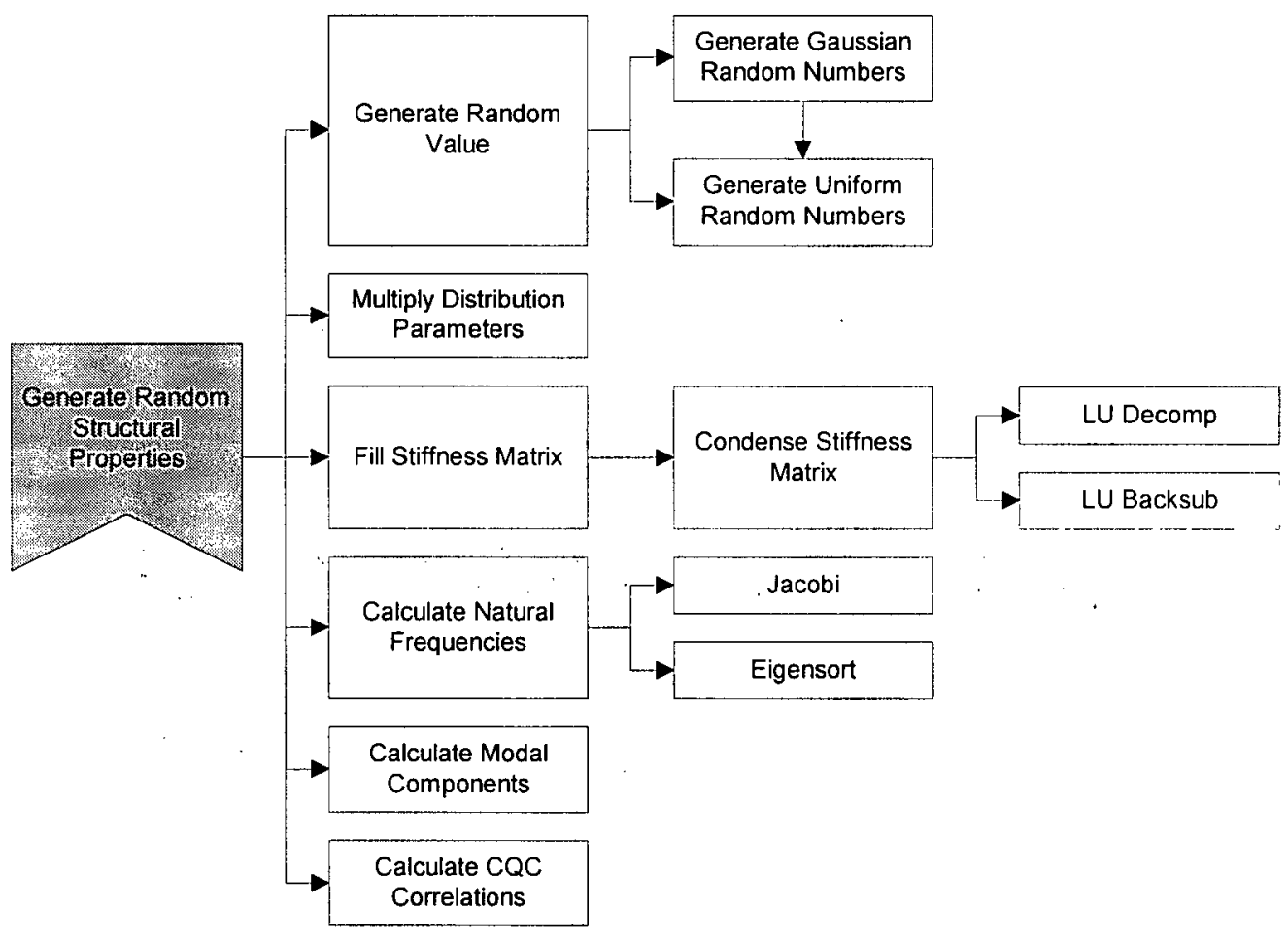

Figure 4.6: Computational Framework - Generate Random Properties

\subsection{SOFTWARE FEATURES}

The user-interface, outlined schematically in Figure 4.7, is best illustrated using screen captures of the dialog boxes and onscreen plots available to the user as well as sample printouts of dynamic analysis results. These screen captures along with explanatory notes, which are shown in the following sections without further comment, will serve to present the key features of the dialog boxes and the analysis options available. 


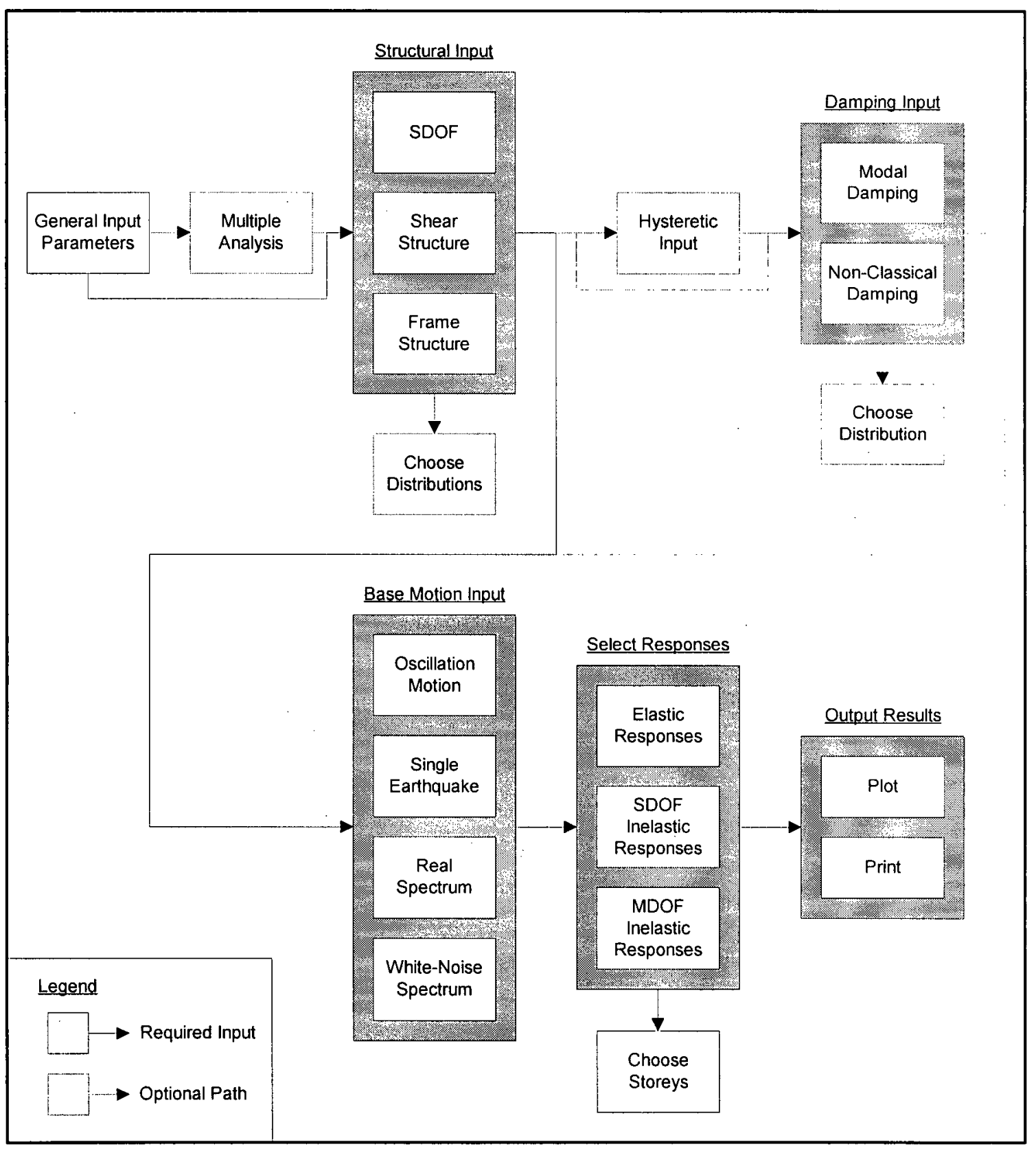

Figure 4.7: User-Interface Framework 


\subsubsection{General Input Parameters Dialog Box}

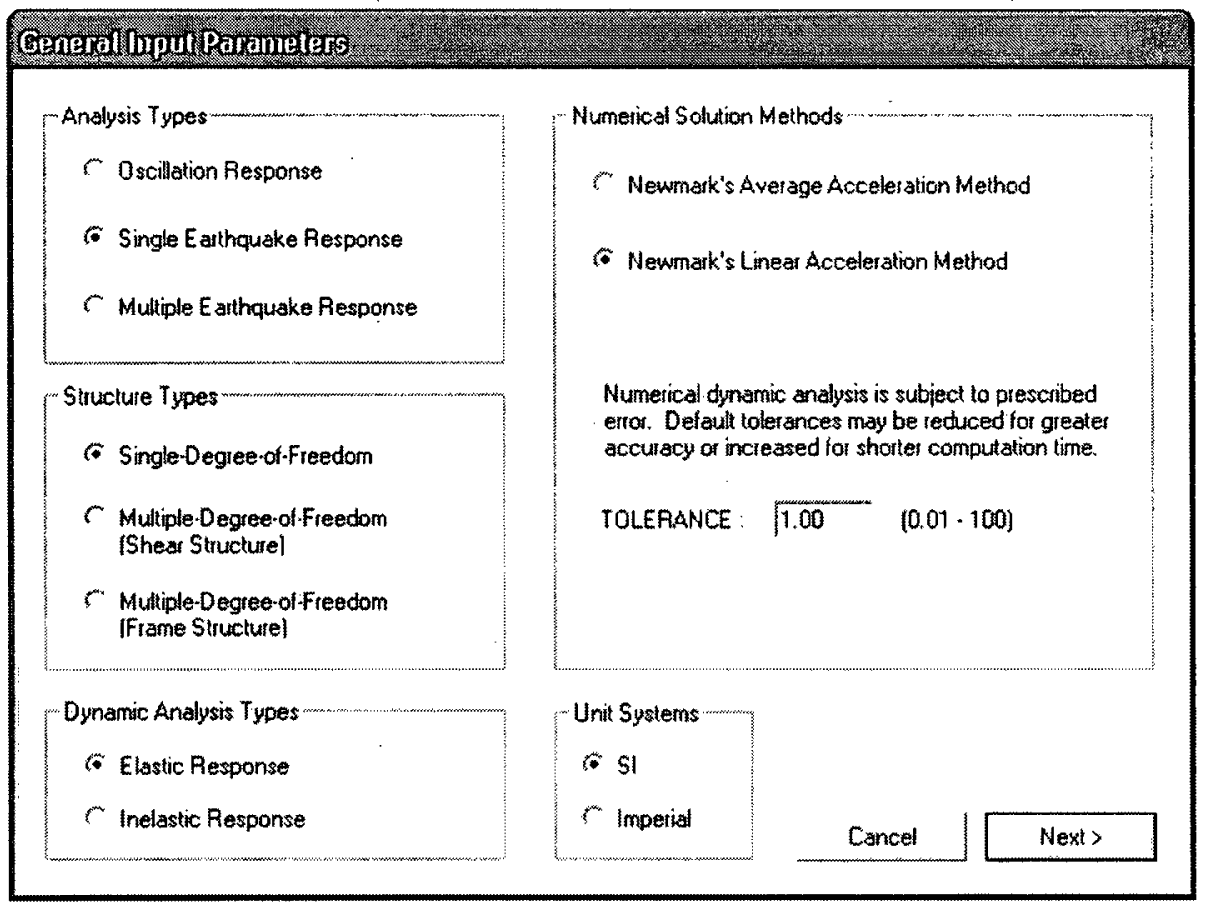

Figure 4.8: General Input Parameters Dialog Box

\begin{tabular}{|c|l|}
\hline Option & \multicolumn{1}{|c|}{ Notes } \\
\hline Oscillation Response & $\begin{array}{l}\text { Input motion is specified as a superposition of sine waves for } \\
\text { structural and connection test protocols. }\end{array}$ \\
\hline Single Earthquake Response & $\begin{array}{l}\text { Detailed response analysis of a single earthquake including } \\
\text { response spectra option and onscreen response time-history plots. }\end{array}$ \\
\hline Multiple Earthquake Response & Summary level response results for multiple earthquakes. \\
\hline Single-Degree-of-Freedom & 1-D structure with fixed base. \\
\hline $\begin{array}{c}\text { Multiple-Degree-of-Freedom } \\
\text { (Shear) }\end{array}$ & 2-D structure with rigid floor diaphragms. \\
\hline $\begin{array}{c}\text { Multiple-Degree-of-Freedom } \\
\text { (Frame) }\end{array}$ & 2-D structure with flexible floor beams. \\
\hline Elastic Response & Elastic response is assumed regardless of displacement. \\
\hline $\begin{array}{c}\text { Newmark Avesponse } \\
\text { Method }\end{array}$ & Inelastic response is modeled using input hysteretic data. \\
\hline $\begin{array}{c}\text { Newmark Linear Acceleration } \\
\text { Method }\end{array}$ & $\begin{array}{l}\text { Unconditionally stable numerical solution method. This method } \\
\text { is automatically selected for inelastic MDOF analysis. }\end{array}$ \\
\hline $\begin{array}{l}\text { Default numerical solution method. } \\
\text { Tolerance }\end{array}$ & $\begin{array}{l}\text { Tolerance relative to encoded default values. This field is used to } \\
\text { multiply the default values. }\end{array}$ \\
\hline
\end{tabular}




\subsubsection{Multiple Earthquake Analysis Parameters Dialog Box}

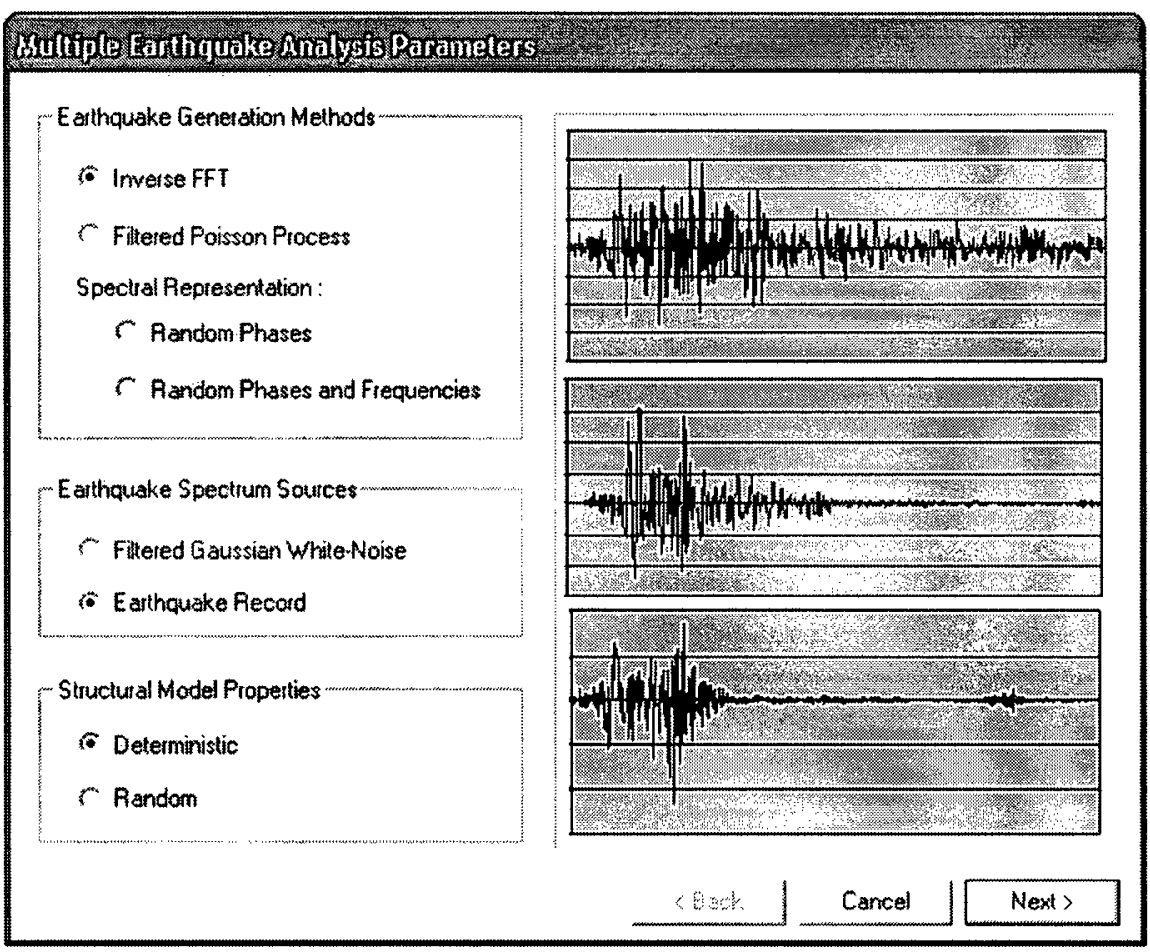

Figure 4.9: Multiple Earthquake Analysis Parameters Dialog Box

\begin{tabular}{|c|l|}
\hline Option & \multicolumn{1}{|c|}{ Notes } \\
\hline Inverse FFT & $\begin{array}{l}\text { Earthquakes are generated using an inverse Fast Fourier Transform } \\
\text { process where the components of the input earthquake frequency } \\
\text { spectrum are randomized with uniform random numbers. }\end{array}$ \\
\hline Filtered Poisson Process & $\begin{array}{l}\text { Generated earthquakes are modeled as the sum of a series of } \\
\text { independent impulses arriving at Poisson distributed times. The } \\
\text { independent pulses have random frequencies with a probability } \\
\text { density based on the spectral density of the input earthquake record. } \\
\text { Phase angles of the independent pulses are uniformly randomly } \\
\text { distributed from 0 } \rightarrow 2 \pi .\end{array}$ \\
\hline Random Phases & $\begin{array}{l}\text { Earthquakes are generated using the Spectral Representation } \\
\text { method with harmonic frequency phases uniformly randomly } \\
\text { distributed from } 0 \rightarrow 2 \pi \text { All generated earthquakes have an } \\
\text { autocorrelation function that matches the input earthquake record. }\end{array}$ \\
\hline Random Phases and Frequencies & $\begin{array}{l}\text { Same as above except the harmonic frequencies are also random } \\
\text { variables with a probability density based on the spectral density of } \\
\text { the input earthquake record. This tends to concentrate random } \\
\text { frequencies around the peak of the input power spectrum. }\end{array}$ \\
\hline Filtered Gaussian White-Noise & $\begin{array}{l}\text { The input spectral density for generating earthquakes is constructed } \\
\text { by shaping a constant white-noise spectrum with a low-pass } \\
\text { Kanai-Tajimi filter and high-pass Clough-Penzien filter. }\end{array}$ \\
\hline Earthquake Record & The input spectral density for generating earthquakes is taken from \\
\hline
\end{tabular}




\begin{tabular}{|c|l|}
\hline & the calculated power spectrum of an input earthquake record. \\
\hline Deterministic & All structural properties and damping values are constant. \\
\hline Random & $\begin{array}{l}\text { Structural properties and damping values are random variables } \\
\text { following a selected probability distribution. A random structure is } \\
\text { generated for each earthquake response analysis. }\end{array}$ \\
\hline
\end{tabular}

\subsubsection{Single-Degree-of-Freedom Properties Dialog Box}

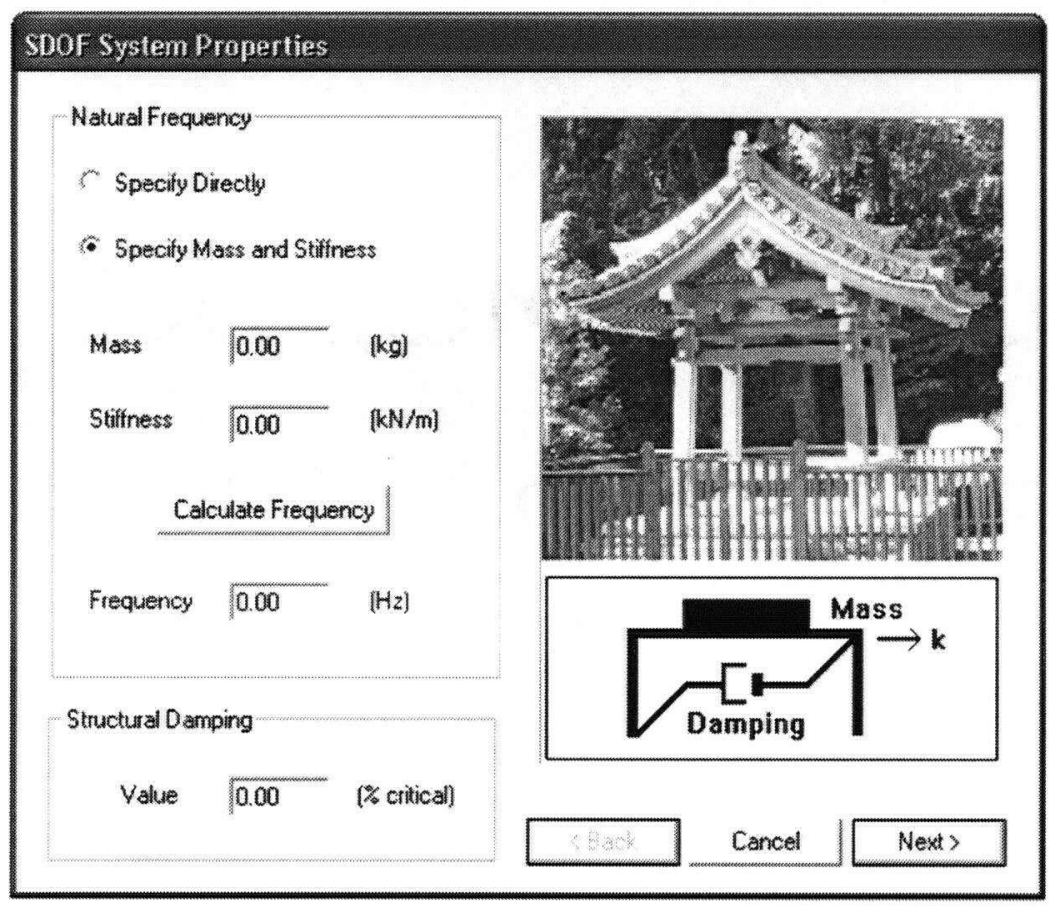

Figure 4.10: Single-Degree-of-Freedom Properties Dialog Box

\begin{tabular}{|c|l|}
\hline Option & \multicolumn{1}{c|}{ Notes } \\
\hline Specify Directly & $\begin{array}{l}\text { The natural frequency of the structure is input directly. This option will } \\
\text { disable the Mass and Stiffness fields and Calculate Frequency button. }\end{array}$ \\
\hline Specify Mass and Stiffness & $\begin{array}{l}\text { The natural frequency of the structure is determined from input values } \\
\text { for mass and stiffness. The Calculate Frequency button updates the } \\
\text { natural frequency. }\end{array}$ \\
\hline
\end{tabular}




\subsubsection{Multiple-Degree-of-Freedom Shear Structure Properties Dialog Box}

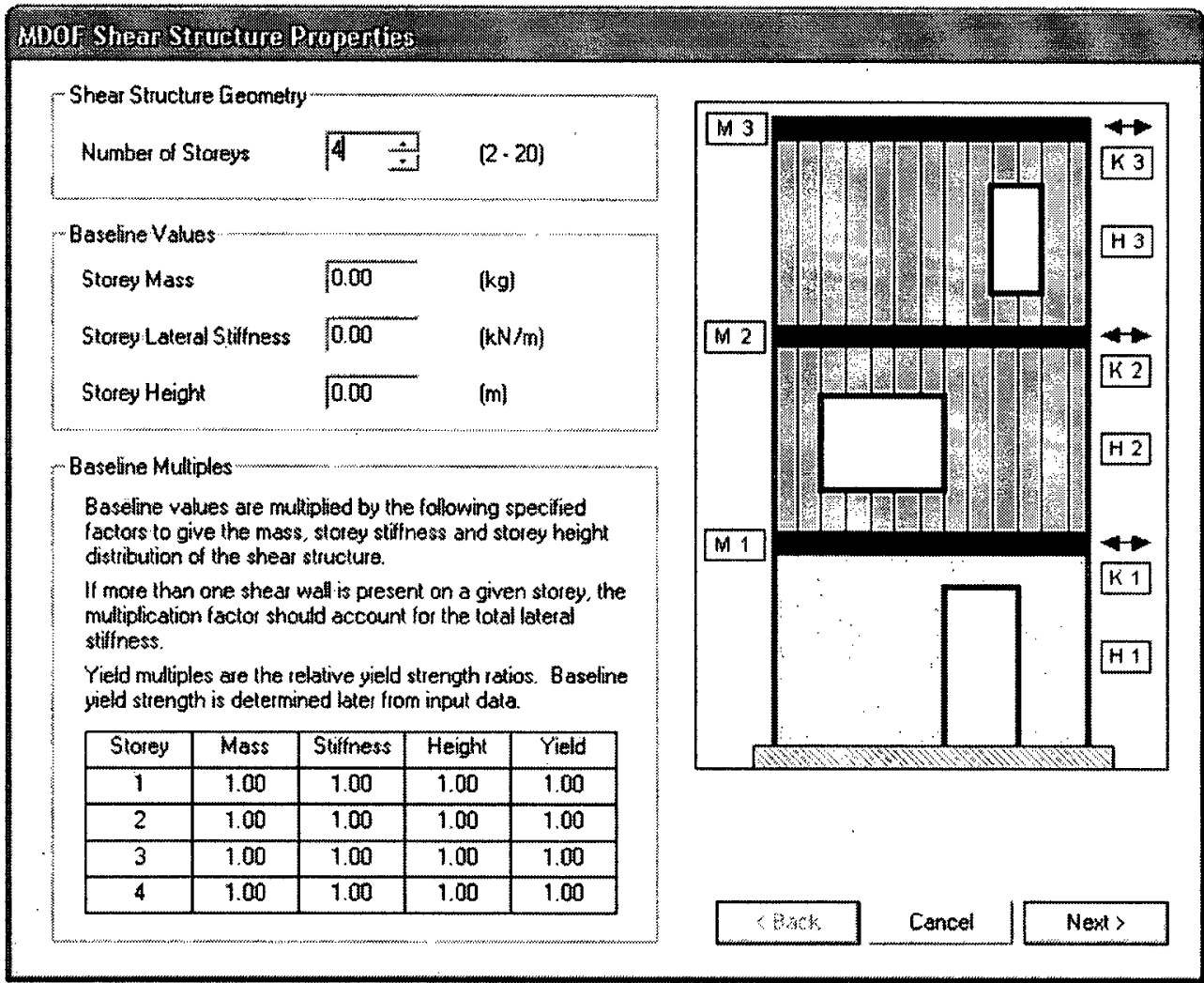

Figure 4.11: Multiple-Degree-of-Freedom Shear Structure Properties Dialog Box

\begin{tabular}{|c|l|}
\hline Field & \multicolumn{1}{|c|}{ Notes } \\
\hline Baseline Values & $\begin{array}{l}\text { Baseline values for storey mass, storey lateral stiffness and storey height are used } \\
\text { to allow a regular structure to be quickly specified as a pattern of baseline } \\
\text { multiples. }\end{array}$ \\
\hline \multirow{3}{*}{ Baseline Multiples } & $\begin{array}{l}\text { Baseline values are multiplied by the specified baseline multiples that default to } \\
1.00 \text {. Yield multiples are the relative yield strengths of each storey in which the } \\
\text { baseline yield strength is determined later from input hysteretic data. The Yield } \\
\text { column is disabled for elastic analysis. }\end{array}$ \\
\hline
\end{tabular}




\subsubsection{Multiple-Degree-of-Freedom Frame Structure Properties Dialog Boxes}
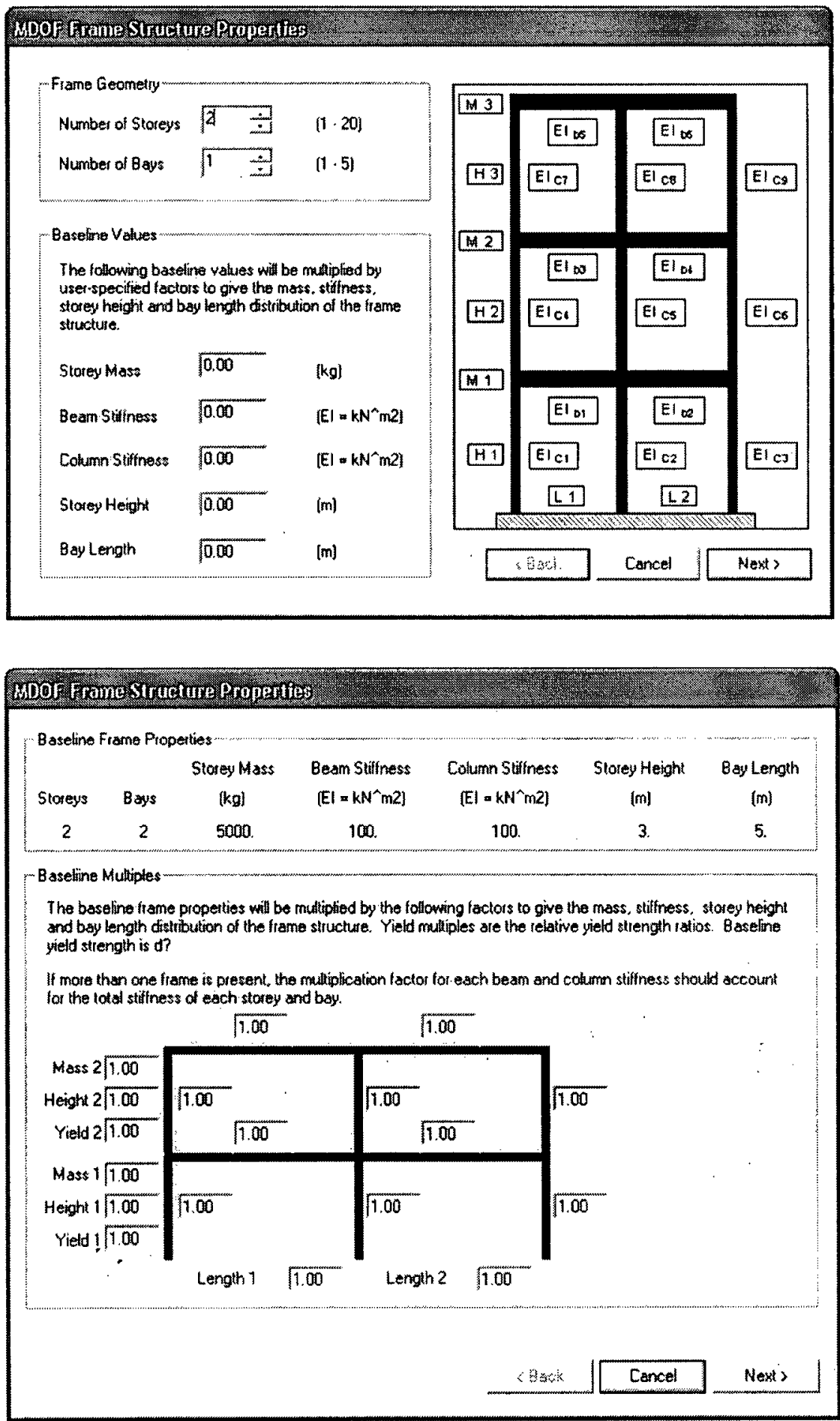

Figure 4.12: Multiple-Degree-of-Freedom Frame Structure Properties Dialog Boxes 


\begin{tabular}{|c|l|}
\hline Field & \multicolumn{1}{|c|}{ Notes } \\
\hline Baseline Values & $\begin{array}{l}\text { Baseline values for storey mass, beam stiffness, column stiffness, storey height } \\
\text { and bay length are used to allow a regular structure to be quickly specified as a } \\
\text { pattern of baseline multiples. }\end{array}$ \\
\hline \multirow{3}{*}{ Baseline Multiples } & $\begin{array}{l}\text { Baseline values are multiplied by the specified baseline multiples that default to } \\
1.00 \text { To simplify the specification of baseline multiples, the input fields are } \\
\text { arranged within a schematic of the frame structure. Yield multiples are the } \\
\text { relative yield strengths of each storey in which the baseline yield strength is } \\
\text { determined later from input hysteretic data. The Yield fields are disabled for } \\
\text { elastic analysis. }\end{array}$ \\
\hline
\end{tabular}

\subsubsection{Probability Distribution Example Dialog Box}

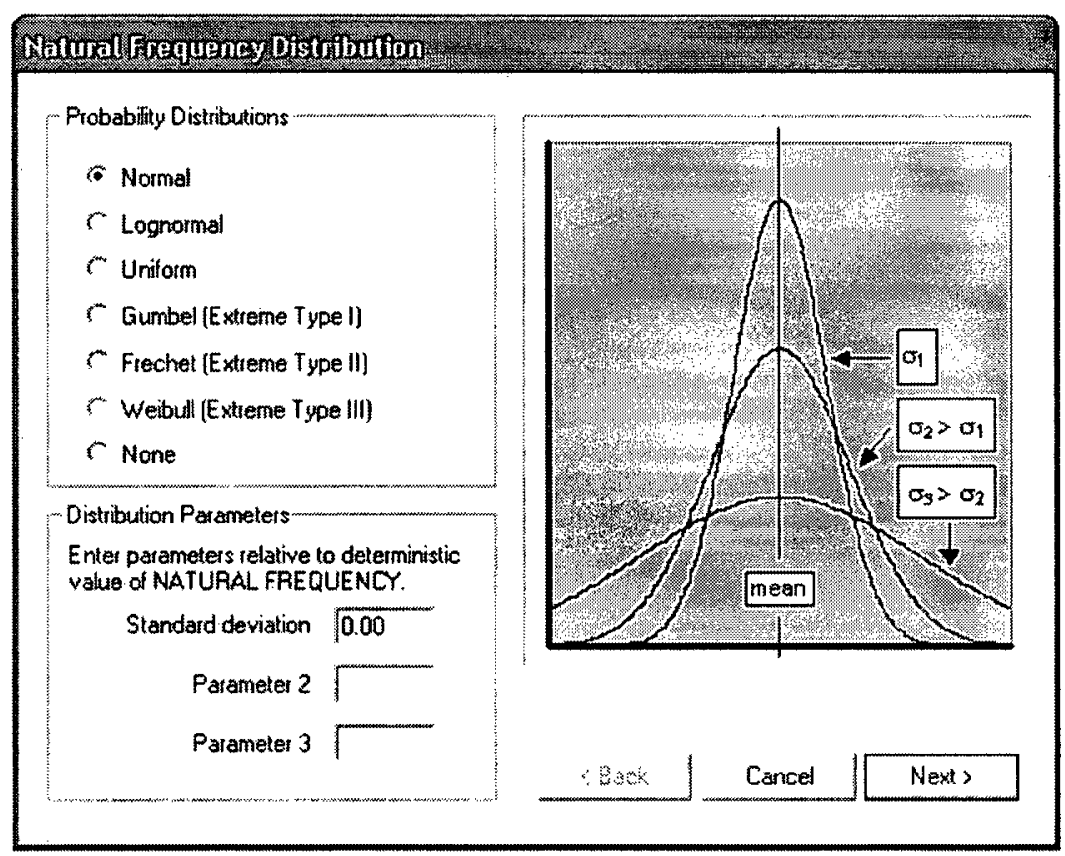

Figure 4.13: Probability Distribution Example Dialog Box

\begin{tabular}{|c|l|}
\hline Option & \multicolumn{1}{|c|}{ Notes } \\
\hline Probability Distributions & $\begin{array}{l}\text { A Probability Distribution dialog box will appear for each structural } \\
\text { parameter that applies to the current analysis. For example, it will appear } \\
\text { twice for a SDOF system that is specified by natural frequency, once for the } \\
\text { natural frequency and once for the specified damping. }\end{array}$ \\
\hline Distribution Parameters & $\begin{array}{l}\text { The parameter fields specify the shape characteristics of the chosen } \\
\text { probability distribution. Values should be entered with respect to the } \\
\text { baseline structural properties entered previously, in the chosen unit system. } \\
\text { In the case of modal damping, parameter values are entered with respect to } \\
\text { the lowest selected mode of response. The mean value of the distribution is } \\
\text { taken as the baseline value. }\end{array}$ \\
\hline
\end{tabular}




\subsubsection{Hysteresis Parameter Identification Dialog Boxes}
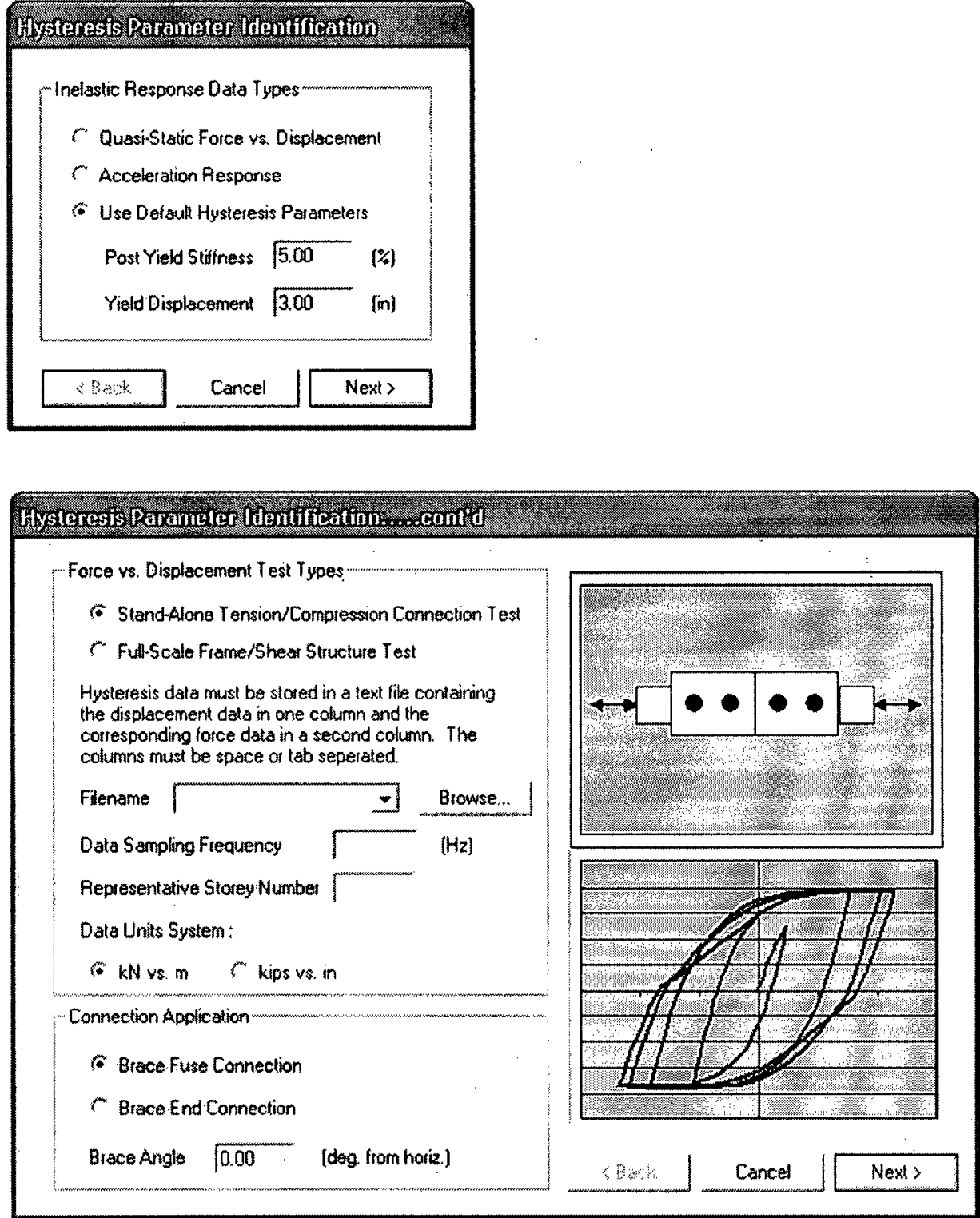

Figure 4.14: Hysteresis Parameter Identification Dialog Box (Quasi-Static Data)

\begin{tabular}{|c|l|}
\hline Option/Field & \multicolumn{1}{|c|}{ Notes } \\
\hline Quasi-Static Force vs. Displacement & $\begin{array}{l}\text { Hysteresis parameter identification is based on pseudo-static } \\
\text { cyclic displacement test data of an SDOF structural system or } \\
\text { connection. }\end{array}$ \\
\hline Acceleration Response & $\begin{array}{l}\text { Hysteresis parameter identification is based on acceleration } \\
\text { response data taken from a shake-table test of an SDOF } \\
\text { structure. }\end{array}$ \\
\hline
\end{tabular}




\begin{tabular}{|c|c|}
\hline Use Default Hysteresis Parameters & $\begin{array}{l}\text { Post-yield stiffness is specified as a percentage of initial } \\
\text { stiffness. Yield displacement may be specified as any value } \\
\text { greater than zero. A yield displacement equivalent to the peak } \\
\text { elastic displacement response will result in a slightly different } \\
\text { dynamic response, as compared to the elastic response, due to } \\
\text { the continuous nonlinear nature of the smooth hysteresis model. } \\
\text { Hysteresis loops are assumed to remain undamaged with no } \\
\text { pinching and no progressive strength or stiffness degradation. } \\
\text { For MDOF structures, the specified yield displacement applies } \\
\text { to the first storey. }\end{array}$ \\
\hline $\begin{array}{c}\text { Stand-Alone Tension/Compression } \\
\text { Test }\end{array}$ & $\begin{array}{l}\text { The dialog box with this option will appear if the Quasi- } \\
\text { Static... option is selected from the previous dialog box. It is } \\
\text { assumed that the specified cyclic displacement data is taken } \\
\text { from a connection test in which the brace-type connection is } \\
\text { subjected to cyclic tension and compression forces. Choosing } \\
\text { this option will enable the Connection Application options at the } \\
\text { bottom of the dialog box. }\end{array}$ \\
\hline $\begin{array}{l}\text { Full-Scale Frame/Shear Structure } \\
\text { Test }\end{array}$ & $\begin{array}{l}\text { It is assumed that the specified cyclic displacement test data } \\
\text { corresponds to the lateral displacement of the top of a full-scale } \\
\text { frame or shear wall. Choosing this option will disable the } \\
\text { Connection Application options at the bottom of the dialog box. }\end{array}$ \\
\hline Filename & $\begin{array}{l}\text { Test data must be stored as a text file with displacement data in } \\
\text { one column and the corresponding force data in a second } \\
\text { column. Columns must be space or tab seperated. Maximum } \\
\text { column width is } 12 \text { characters and data may be specified in } \\
\text { exponential format. }\end{array}$ \\
\hline Data Sampling Frequency & $\begin{array}{l}\text { Number of pseudo-static force vs. displacement data points } \\
\text { recorded per second. }\end{array}$ \\
\hline Representative Storey Number & $\begin{array}{l}\text { This field is enabled when an MDOF structure has been } \\
\text { specified for dynamic analyses. The representative storey } \\
\text { number indicates which frame or shear wall stiffness and yield } \\
\text { strength multiple to associate with the identified hysteresis } \\
\text { parameters. The hysteresis parameters for the other storeys are } \\
\text { adjusted according to the storey stiffnesses and yield strength } \\
\text { multiples specified for the other storeys. }\end{array}$ \\
\hline Data Units System & Units system of the input hysteretic data. \\
\hline Brace Fuse Connection & $\begin{array}{l}\text { Brace with a single yielding element or connection, typically at } \\
\text { mid-brace. All inelastic deformation is concentrated at this one } \\
\text { point. Lateral displacement of the top of the frame and the } \\
\text { corresponding lateral force are determined by adjusting the } \\
\text { input data according to the input brace angle. }\end{array}$ \\
\hline Brace End Connection & $\begin{array}{l}\text { Same as above except the brace has yielding end connections. } \\
\text { Therefore, lateral displacement is calculated as twice the } \\
\text { adjusted input displacement to account for two yielding } \\
\text { connections. }\end{array}$ \\
\hline Brace Angle & Angle of brace in degrees from horizontal. \\
\hline
\end{tabular}




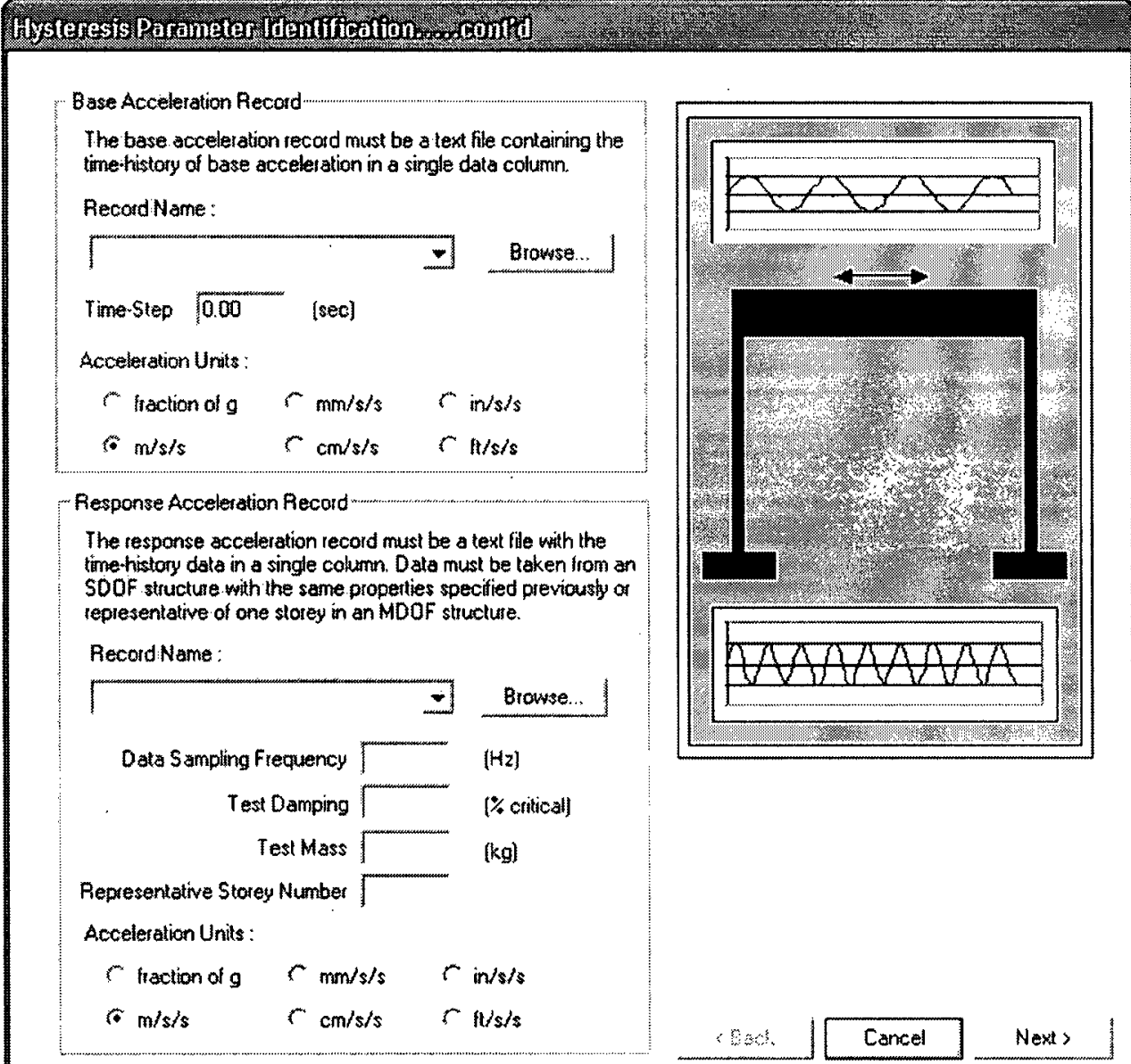

Figure 4.15: Hysteresis Parameter Identification Dialog Box (Acceleration Data)

\begin{tabular}{|c|l|}
\hline Option/Field & \multicolumn{1}{|c|}{ Notes } \\
\hline Base Acceleration Record & $\begin{array}{l}\text { The dialog box with this option will appear if the Acceleration } \\
\text { Response option is selected from the previous dialog box. The base } \\
\text { acceleration record must be stored as a text file containing the time- } \\
\text { history data in a single column. Maximum column width is 12 } \\
\text { characters and data may be specified in exponential format. }\end{array}$ \\
\hline Time-Step & $\begin{array}{l}\text { Time span between base acceleration time-history data points. The } \\
\text { inverse of the time-step must be an integer multiple of the response } \\
\text { acceleration data sampling frequency. }\end{array}$ \\
\hline Acceleration Units & Unit system of input base acceleration record. \\
\hline Response Acceleration Record & $\begin{array}{l}\text { The response acceleration record must be stored as a text file } \\
\text { containing the time--history data in a single column. Data must be } \\
\text { taken from an SDOF structure with the properties specified earlier or } \\
\text { representative of one storey in an MDOF structure. Maximum } \\
\text { column width is 12 characters and data may be specified in } \\
\text { exponential format. }\end{array}$ \\
\hline Data Sampling Frequency & $\begin{array}{l}\text { Number of response acceleration time-history data points recorded } \\
\text { per second. The data sampling frequency must be an integer multiple }\end{array}$ \\
\hline
\end{tabular}




\begin{tabular}{|c|l|}
\hline & of the inverse of the base acceleration time-step. \\
\hline Test Damping & $\begin{array}{l}\text { Estimated damping of the SDOF test structure. This damping level } \\
\text { does not have to match the modal or storey damping level(s) } \\
\text { specified for the structural system being modeled with the identified } \\
\text { hysteresis parameters. }\end{array}$ \\
\hline Test Mass & $\begin{array}{l}\text { This field is enabled when an MDOF structure has been specified for } \\
\text { dynamic analyses. The test mass is defined as the gravity load placed } \\
\text { on the SDOF test structure for shake-table testing. It is used to } \\
\text { determine the natural frequency of the test structure for response } \\
\text { analysis in the hysteresis parameter identification process. }\end{array}$ \\
\hline Representative Storey Number & $\begin{array}{l}\text { This field is enabled when an MDOF structure has been specified for } \\
\text { dynamic analyses. The representative storey number indicates which } \\
\text { frame or shear wall stiffness and yield strength multiple to associate } \\
\text { with the identified hysteresis parameters. The hysteresis parameters } \\
\text { for the other storeys are adjusted according to the storey stiffnesses } \\
\text { and yield strength multiples specified for the other storeys. }\end{array}$ \\
\hline Acceleration Units & Unit system of input response acceleration record. \\
\hline
\end{tabular}

\subsubsection{Modal Damping Parameters Dialog Box}

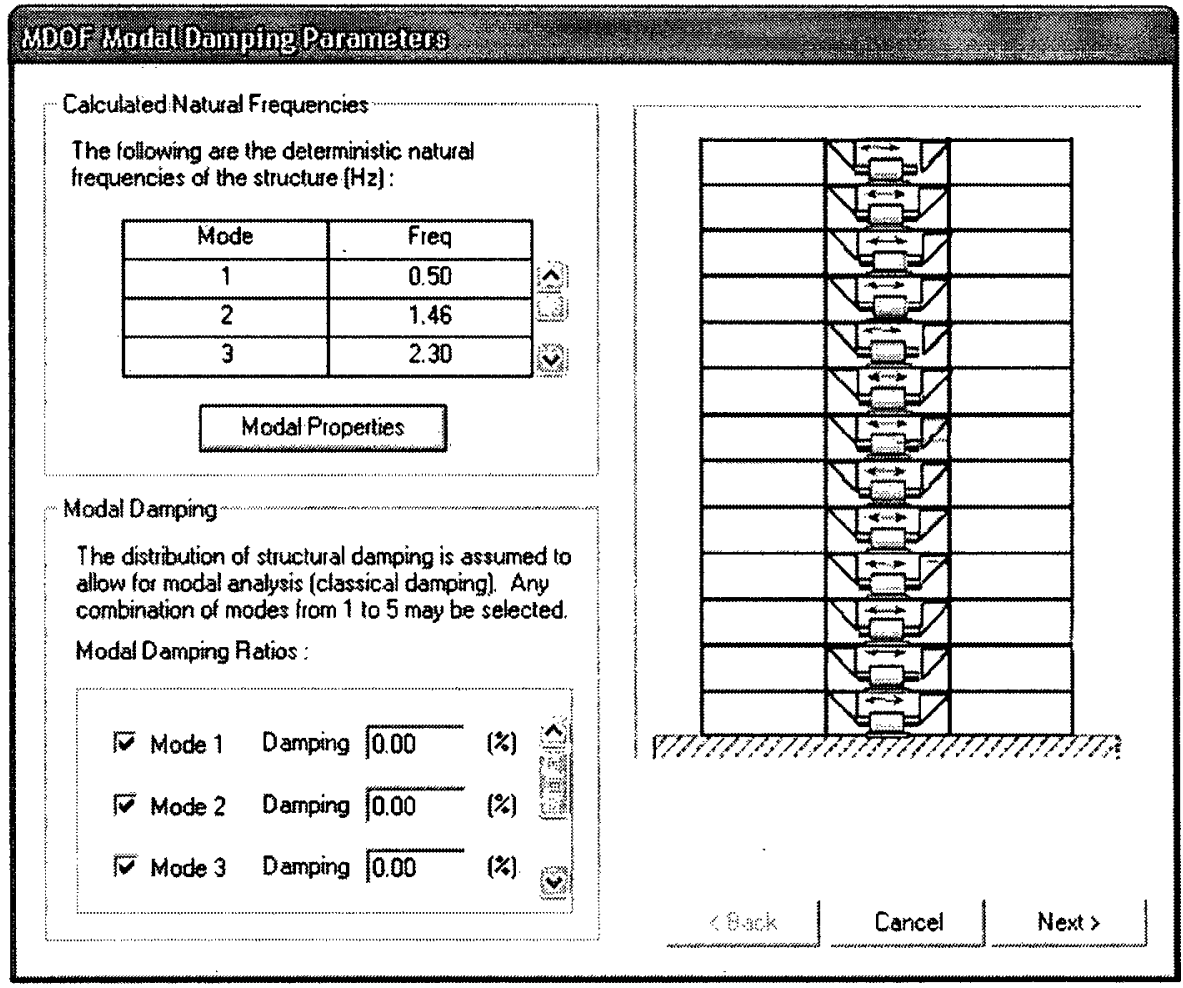

Figure 4.16: Modal Damping Parameters Dialog Box 


\begin{tabular}{|c|l|}
\hline Field & \multicolumn{1}{|c|}{ Notes } \\
\hline Calculated Natural Frequencies & $\begin{array}{l}\text { Calculated natural frequencies for translational modes only. } \\
\text { These frequencies, which are given in Hz, are determined from } \\
\text { the baseline structural properties and baseline multiples that were } \\
\text { specified previously. }\end{array}$ \\
\hline Modal Properties Button & $\begin{array}{l}\text { Generates a pop-up dialog box that displays mode shape data, } \\
\text { modal expansion of floor masses, effective modal masses and } \\
\text { effective modal heights. See Figure 4.17. }\end{array}$ \\
\hline Modal Damping Ratios & $\begin{array}{l}\text { Modes to be included in the elastic response analysis are selected } \\
\text { from a scroll box along with a corresponding damping level for } \\
\text { each selected mode. If structural properties have been specified } \\
\text { as random, a Probability Distribution dialog box will follow this } \\
\text { dialog box. The parameters for the selected damping probability } \\
\text { distribution should be entered relative to the lowest selected } \\
\text { mode. }\end{array}$ \\
\hline
\end{tabular}

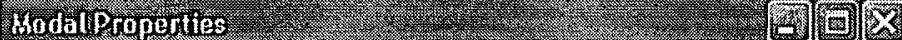

Mode shapes normalized to unily:
(Modes in column ordered hom top storey to boltom
$\begin{array}{llllll}1.0000 & -0.9190 & 0.7635 & -0.5462 & 0.2846 \\ 0.9190 & -0.2846 & 0.5462 & 1.0000 & -0.7635 \\ 0.7635 & 0.5462 & -0.9190 & -0.2846 & 1.0000 \\ 0.5462 & 1.0000 & 0.2846 & -0.7635 & -0.9190 \\ 0.2846 & 0.7635 & 1.0000 & 0.9190 & 0.5462\end{array}$

Mode shapes nomalized to give unil generalized modal masses (Modes in columns ordered from top storey to bottom storey)

$\begin{array}{lllll}0.0028 & 0.0026 & -0.0021 & -0.0015 & 0.0008\end{array}$

$\begin{array}{lllll}0.0026 & 0.0008 & 0.0015 & 0.0028 & .0 .0021\end{array}$

$\begin{array}{lllll}0.0021 & -0.0015 & 0.0026 & -0.0008 & 0.0028\end{array}$

$\begin{array}{lllll}0.0015 & -0.0028 & -0.0008 & -0.0021 & -0.0026\end{array}$

$\begin{array}{lllll}0.0008 & -0.0021 & -0.0028 & 0.0026 & 0.0015\end{array}$

Modal expansion of floor masses (kips/g)

(Modes in columns ordered from top siojey to bottom storey)

$\begin{array}{lllll}125.17 & -36.21 & 15.26 & -6.32 & 1.50\end{array}$

$\begin{array}{lllll}115.03 & -11.22 & .11 .34 & 11.57 & -4.03\end{array}$

$\begin{array}{lllll}95.57 & 21.52 & .19 .09 & -3.29 & 5.28\end{array}$

$\begin{array}{lllll}68.37 & 39.41 & 5.91 & -8.83 & -4.86\end{array}$

$\begin{array}{lllll}35.63 & 30.09 & 20.77 & 10.63 & 2.89\end{array}$

Elfective modal masses (kips/g) : 439.7643 .5912 .113 .750 .78

Elfective modal heights [ $(\mathrm{f}): 42.16 \cdot 14.449 .16 \cdot 7.136 .25$

OK

Figure 4.17: Modal Properties Pop-Up Dialog Box 


\subsubsection{MDOF Damping Parameters Dialog Box}

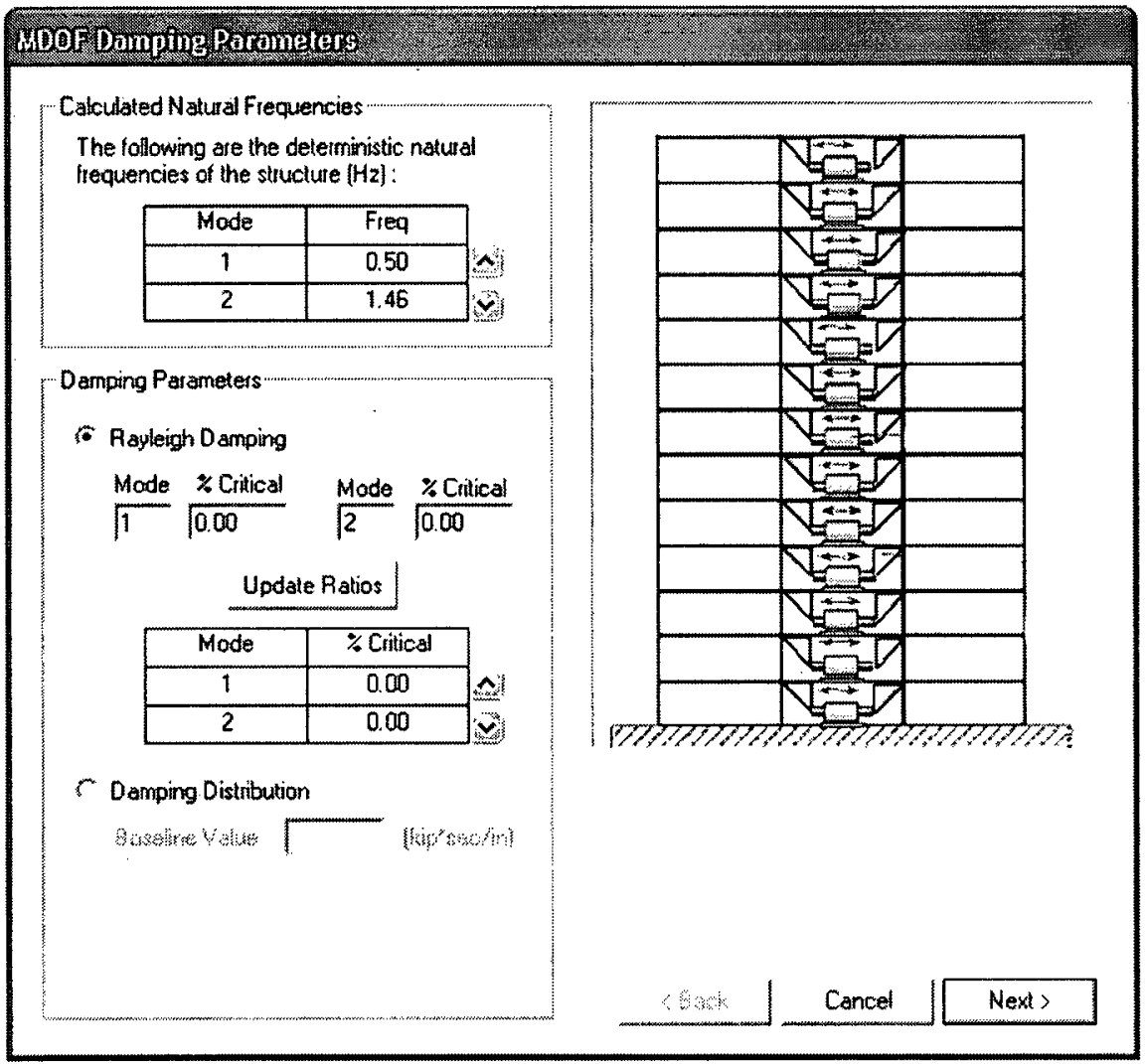

Figure 4.18: MDOF Damping Parameters Dialog Box

\begin{tabular}{|c|l|}
\hline Field/Option & \multicolumn{1}{c|}{ Notes } \\
\hline $\begin{array}{c}\text { Calculated Natural } \\
\text { Frequencies }\end{array}$ & $\begin{array}{l}\text { Calculated natural frequencies for translational modes only. These frequencies are } \\
\text { determined from the baseline structural properties and baseline multiples that } \\
\text { were specified previously. }\end{array}$ \\
\hline \multirow{3}{*}{ Rayleigh Damping } & $\begin{array}{l}\text { Inelastic dynamic analysis precludes modal analysis, which results in the need to } \\
\text { specify the damping matrix. Rayleigh damping is used to construct a classical } \\
\text { damping matrix from modal damping ratios. Two modal damping ratios must be } \\
\text { specified, which then determines the damping ratios for the remaining modes. } \\
\text { The Update Ratios button calculates the damping ratios for each non-specified } \\
\text { mode. If structural properties have been specified as random, a Probability } \\
\text { Distribution dialog box will follow this dialog box. The parameters for the } \\
\text { selected damping probability distribution should be entered relative to the lowest } \\
\text { specified mode. }\end{array}$ \\
\hline Damping & $\begin{array}{l}\text { A non-classical damping matrix is constructed from the specified damping for } \\
\text { each storey. If structural properties have been specified as random, the } \\
\text { parameters for the selected damping probability distribution should be entered } \\
\text { relative to the specified baseline value. }\end{array}$ \\
\hline
\end{tabular}




\subsubsection{Oscillation Motion Parameters Dialog Box}

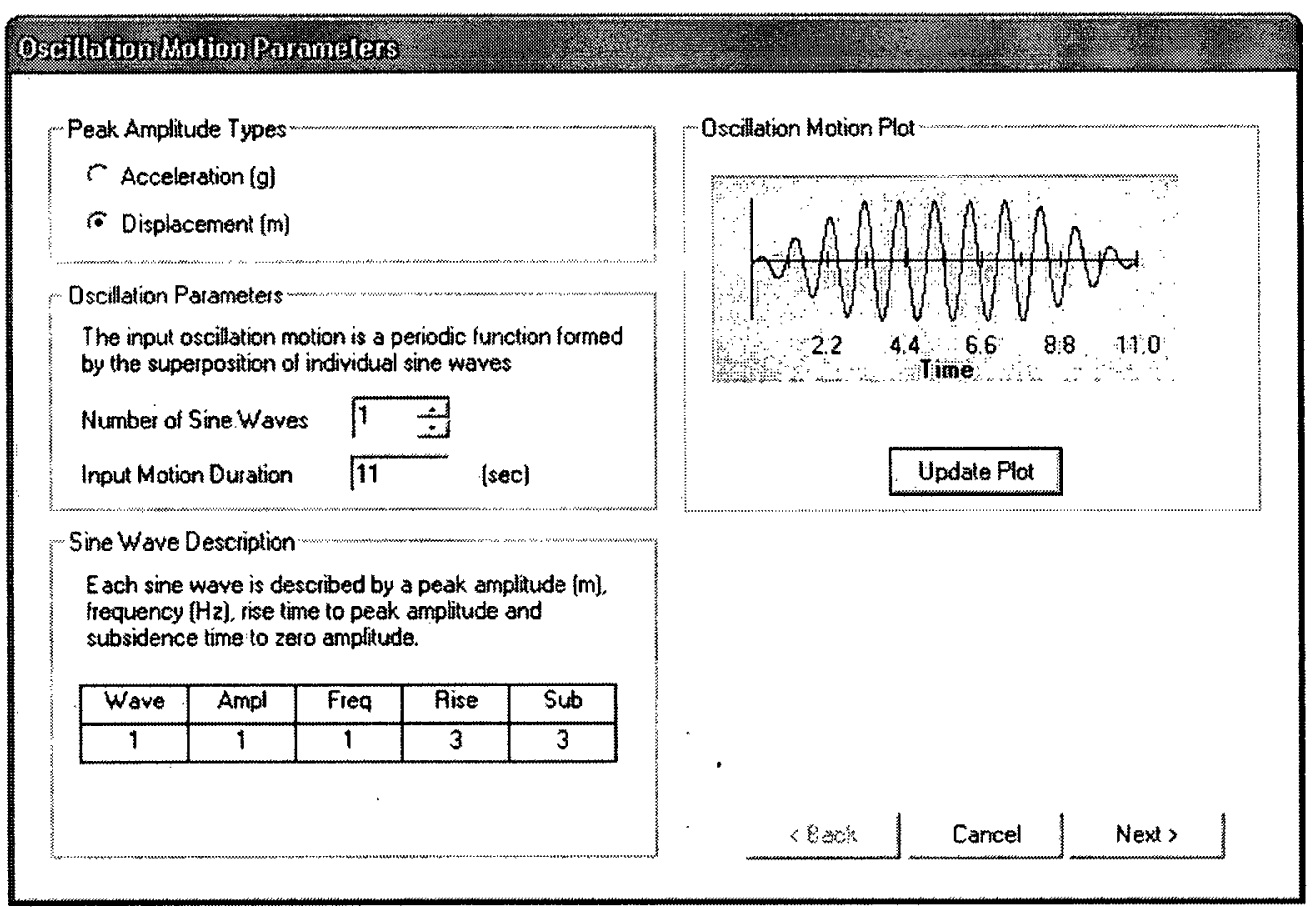

Figure 4.19: Oscillation Motion Parameters Dialog Box

\begin{tabular}{|c|l|}
\hline Field/Option & \multicolumn{1}{|c|}{ Notes } \\
\hline Acceleration & $\begin{array}{l}\text { Base motion is specified as a time-history of acceleration given in } \\
\text { terms of } g .\end{array}$ \\
\hline Displacement & Base motion is specified as a time-history of displacement. \\
\hline Number of Sine Waves & $\begin{array}{l}\text { The input oscillation motion is formed by the superposition of up to } \\
\text { nine sine waves. }\end{array}$ \\
\hline Input Motion Duration & $\begin{array}{l}\text { Duration must be a multiple of 0.01 sec. Maximum duration is } 99 \\
\text { seconds. }\end{array}$ \\
\hline Ampl & $\begin{array}{l}\text { Amplitude of sine wave. This is specified in the chosen unit system } \\
(m, \text { in. or } g)\end{array}$ \\
\hline Freq & Frequency of sine wave in Hz. \\
\hline Rise & $\begin{array}{l}\text { Time when peak amplitude is reached. Default is zero, indicating no } \\
\text { rise time. }\end{array}$ \\
\hline Sub & $\begin{array}{l}\text { Duration of interval during which amplitude decreases from } \\
\text { maximum to zero at the end of the motion. Default is zero, indicating } \\
\text { no subsidence time. }\end{array}$ \\
\hline Update Plot & Updates and displays the calculated superposition of sine waves. \\
\hline
\end{tabular}




\subsubsection{Single Ground Motion Parameters Dialog Box}
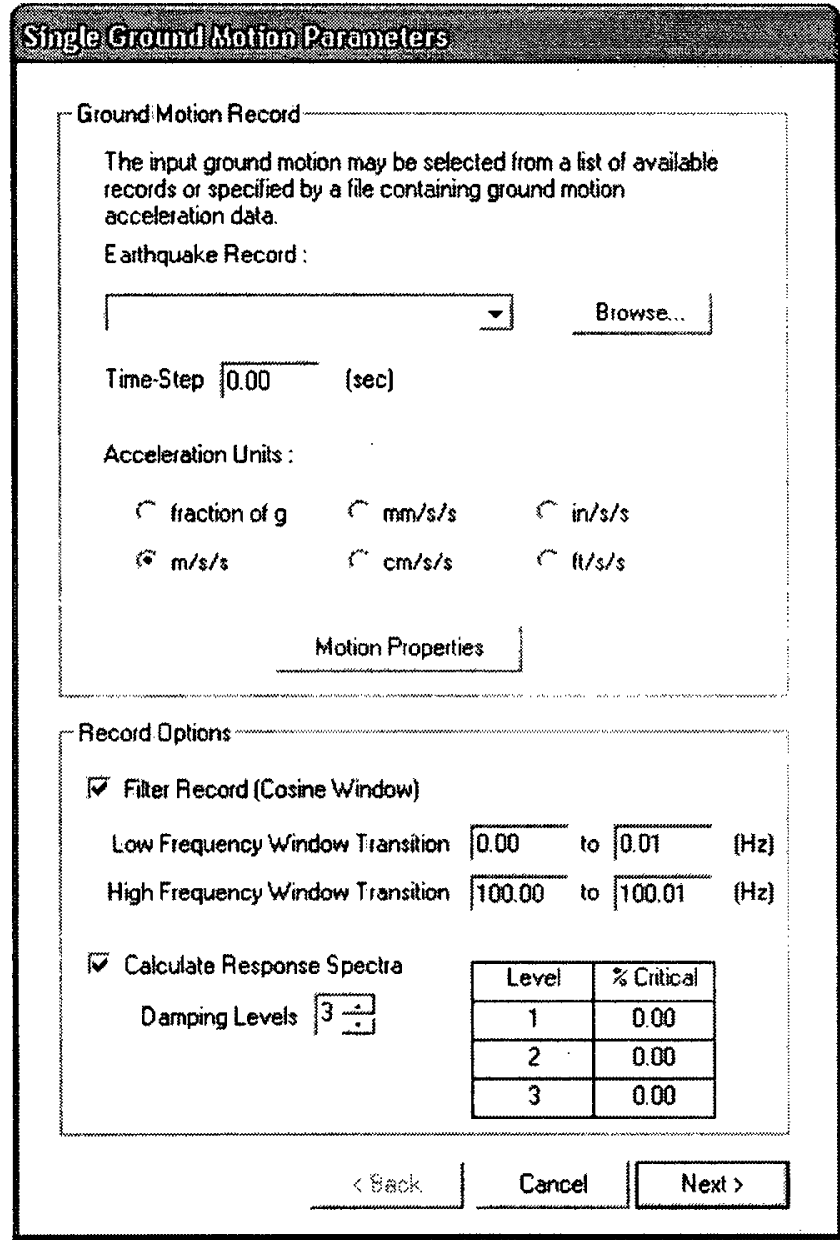

Figure 4.20: Single Ground Motion Parameters Dialog Box

\begin{tabular}{|c|l|}
\hline Field/Option & \multicolumn{1}{|c|}{ Notes } \\
\hline Earthquake Record & $\begin{array}{l}\text { A single ground motion record is used for response analysis and } \\
\text { subsequent onscreen plotting of results. The earthquake record must } \\
\text { be stored as a text file containing the time-history data in a single } \\
\text { column. Maximum column width is } 12 \text { characters and data may be } \\
\text { specified in exponential format. }\end{array}$ \\
\hline Time-Step & Time span between earthquake time-history data points. \\
\hline Acceleration Units & Unit system of earthquake acceleration record. \\
\hline Motion Properties Button & $\begin{array}{l}\text { Generates a pop-up dialog box that displays summary statistics and a } \\
\text { time-history plot of the unfiltered ground motion as well as the } \\
\text { filtered ground motion if the Filter Record option is chosen. See } \\
\text { Figure 4.21. This dialog box may be opened and closed repeatedly to } \\
\text { display the results of adjusting filter window transitions. }\end{array}$ \\
\hline Filter Record & This option enables a cosine-type window filter for removal of high \\
\hline
\end{tabular}




\begin{tabular}{|c|l|}
\hline & $\begin{array}{l}\text { and low frequency content in the earthquake record to ensure that the } \\
\text { ground acceleration record being used for structural dynamic } \\
\text { response analysis is truly representative of a real earthquake. }\end{array}$ \\
\hline $\begin{array}{c}\text { Low Frequency Window } \\
\text { Transition }\end{array}$ & $\begin{array}{l}\text { Specifies the frequency range in which the cosine-window varies } \\
\text { from 0 to 1. Frequencies below the lower limit are removed and } \\
\text { frequencies above the upper limit are unaltered. }\end{array}$ \\
\hline $\begin{array}{c}\text { High Frequency Window } \\
\text { Transition }\end{array}$ & $\begin{array}{l}\text { Specifies the frequency range in which the cosine-window varies } \\
\text { from 1 to 0. Frequencies above the upper limit are removed and } \\
\text { frequencies below the lower limit are unaltered. }\end{array}$ \\
\hline \multirow{5}{*}{$\begin{array}{l}\text { Calculate Response Spectra } \\
\text { specified earthquake record at up to nine damping levels. The } \\
\text { spectra are formed from the maximum elastic response at 351 natural } \\
\text { periods between 0.01 sec and 50 sec. The periods are evenly } \\
\text { distributed in three full decades and a truncated fourth decade defined } \\
\text { as follows: 0.01-0.1 sec, 0.11-1.0 sec, 1.1-10 sec, 10-50 sec. The } \\
\text { spectra are displayed onscreen following response analysis. See } \\
\text { Figure 4.31. }\end{array}$} \\
\hline
\end{tabular}

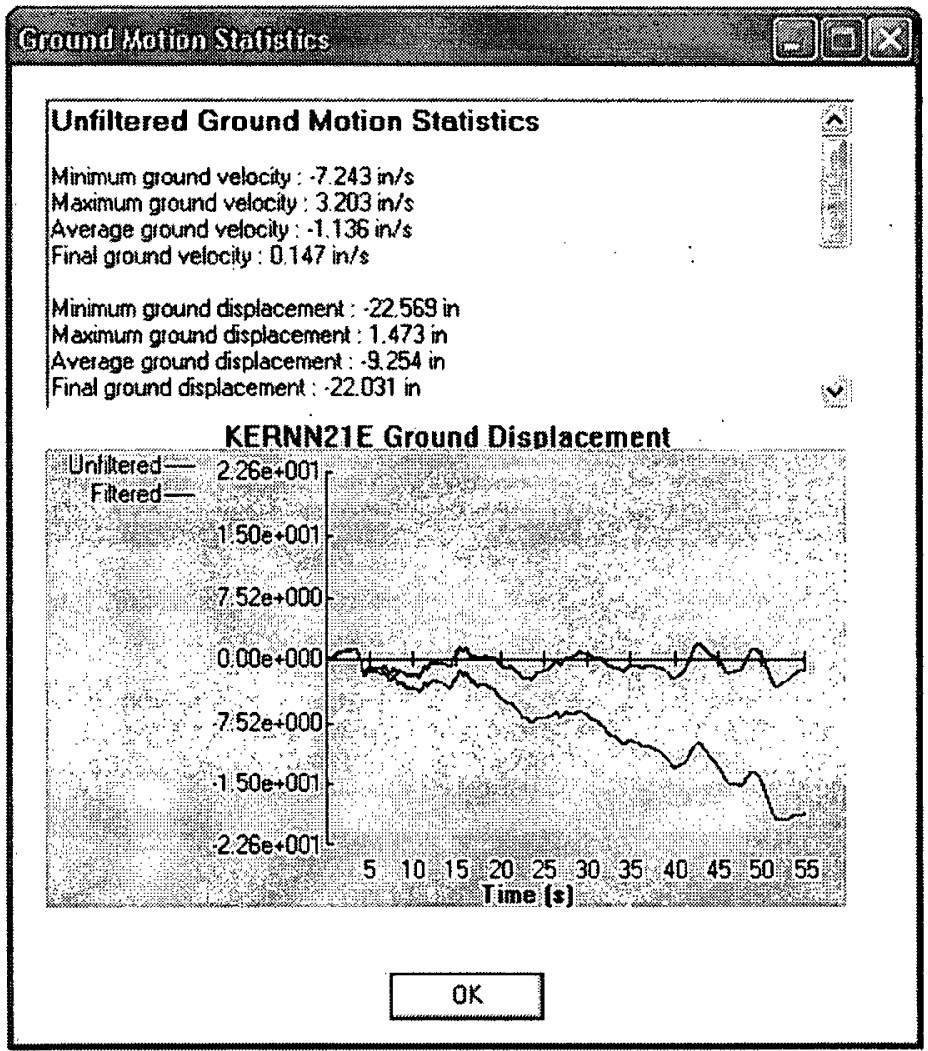

Figure 4.21: Ground Motion Statistics Pop-Up Dialog Box 


\subsubsection{Ground Motion Generation Parameters Dialog Box - Filtered Spectrum}

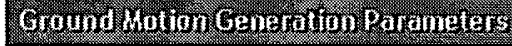

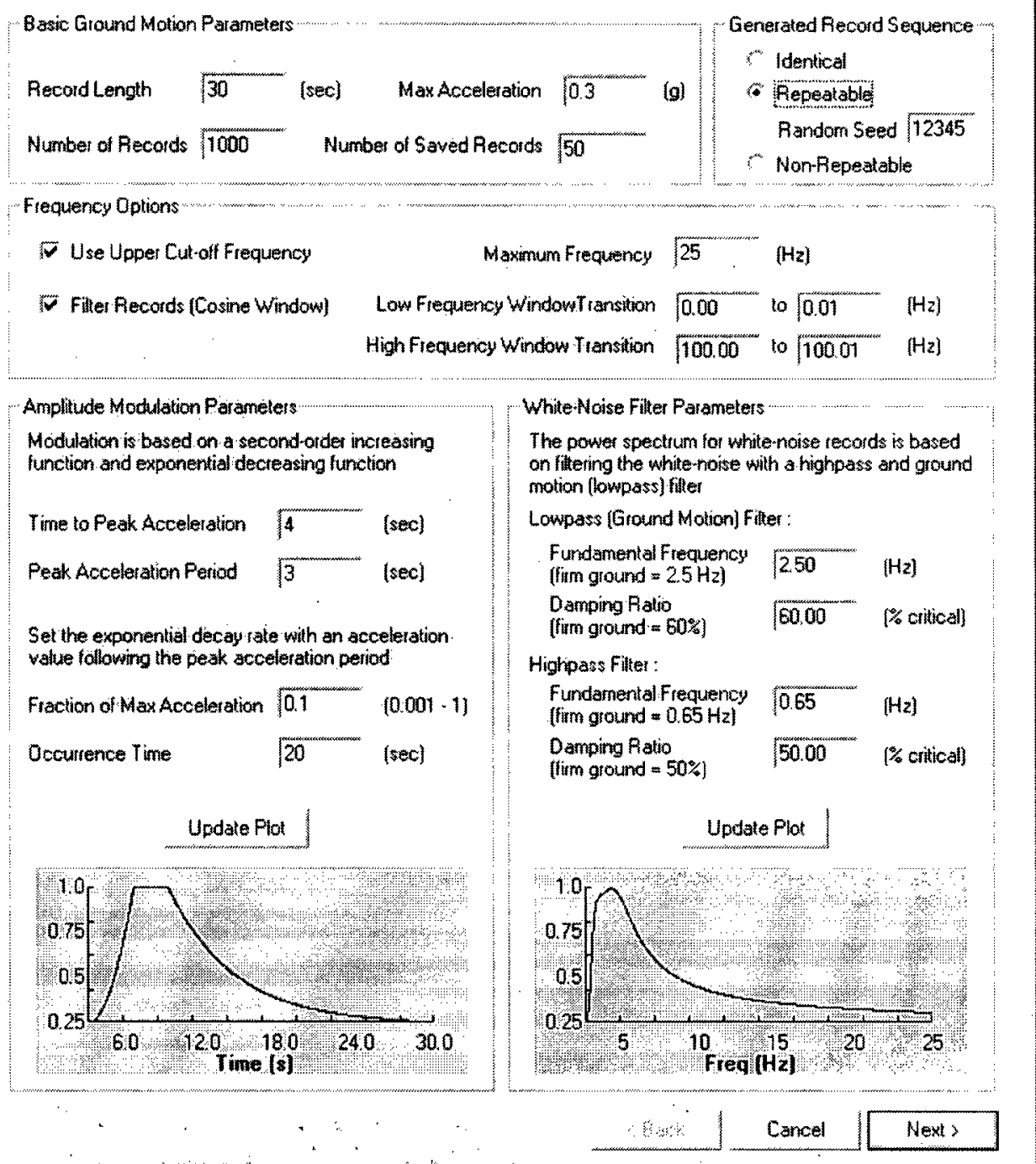

Figure 4.22: Ground Motion Generation Parameters Dialog Box - Filtered Spectrum

\begin{tabular}{|c|l|}
\hline Field/Option & \multicolumn{1}{|c|}{ Notes } \\
\hline Record Length & Duration of earthquake. \\
\hline Maximum Acceleration & Specifies the peak amplitude in the acceleration time-history. \\
\hline Number of Records & Number of generated earthquakes. \\
\hline Number of Saved Records & $\begin{array}{l}\text { Specifies the number of random earthquake time-histories to be } \\
\text { saved in a text file. Maximum number is 50. }\end{array}$ \\
\hline Generated Record Sequence & $\begin{array}{l}\text { This option specifies the random nature of the generated earthquake } \\
\text { records. The three types of record sequences are as follows: } \\
\text { Identical : the same earthquake time-history is used for each }\end{array}$ \\
\hline
\end{tabular}




\begin{tabular}{|c|c|}
\hline & $\begin{array}{l}\text { dynamic analysis. This permits evaluation of the probabilistic } \\
\text { response of structures in which randomness is limited to structural } \\
\text { properties only. } \\
\text { Repeatable : for a given random seed, the same sequence of } \\
\text { earthquake time-histories will be generated each time a multiple } \\
\text { record analysis is done, assuming the same Basic Ground Motion, } \\
\text { Amplitude Modulation and White-Noise Filter parameters are } \\
\text { specified. If structural properties have been specified to be random, } \\
\text { the sequence of random structures is not repeated. This permits } \\
\text { comparison of the relative effect on probabilistic structural response } \\
\text { of earthquake record randomness vs. various degrees of structural } \\
\text { property randomness. } \\
\text { Non-Repeatable : the sequence of generated earthquake time- } \\
\text { histories and the possible sequence of random structural properties } \\
\text { are not repeatable. Specifying this truly random type of multiple } \\
\text { record analysis allows results to be combined with analyses } \\
\text { performed at different times and/or on different computers. }\end{array}$ \\
\hline Use Upper Cut-off Frequency & $\begin{array}{l}\text { This option truncates the input frequency spectrum at the specified } \\
\text { frequency to reduce computation time in generating earthquake } \\
\text { records using the Spectral Representation method or Filtered } \\
\text { Poisson method. This option is disabled when earthquakes are } \\
\text { generated using the inverse Fast Fourier Transform process. }\end{array}$ \\
\hline Filter Records & $\begin{array}{l}\text { This option enables a cosine-type window filter for removal of } \\
\text { high and low frequency content in the earthquake record to ensure } \\
\text { that the ground acceleration record being used for structural } \\
\text { dynamic response analysis is truly representative of a real } \\
\text { earthquake. }\end{array}$ \\
\hline $\begin{array}{l}\text { Low Frequency Window } \\
\text { Transition }\end{array}$ & $\begin{array}{l}\text { Specifies the frequency range in which the cosine-window varies } \\
\text { from } 0 \text { to } 1 \text {. Frequencies below the lower limit are removed and } \\
\text { frequencies above the upper limit are unaltered. }\end{array}$ \\
\hline $\begin{array}{l}\text { High Frequency Window } \\
\text { Transition }\end{array}$ & $\begin{array}{l}\text { Specifies the frequency range in which the cosine-window varies } \\
\text { from } 1 \text { to } 0 \text {. Frequencies above the upper limit are removed and } \\
\text { frequencies below the lower limit are unaltered. }\end{array}$ \\
\hline Time to Peak Acceleration & $\begin{array}{l}\text { Temporal amplitude modulation of the generated ground } \\
\text { acceleration record is based on a second-order increasing function } \\
\text { followed by a period of constant maximum acceleration and then an } \\
\text { exponentially decreasing function. The parameters specifying the } \\
\text { temporal amplitude modulation are treated as deterministic and } \\
\text { therefore are not randomized for each record generated. The } \\
\text { constant maximum acceleration is normalized to unity by the } \\
\text { specified peak acceleration. The time when peak acceleration } \\
\text { amplitude is reached determines the second-order increasing } \\
\text { function. }\end{array}$ \\
\hline Peak Acceleration Period & $\begin{array}{l}\text { Specifies the duration of the constant maximum acceleration period } \\
\text { in the generated earthquake record. }\end{array}$ \\
\hline Fraction of Max Acceleration & $\begin{array}{l}\text { The exponential decay rate in acceleration amplitude is determined } \\
\text { by a single point following the peak acceleration period. The } \\
\text { specified fraction of maximum acceleration sets the amplitude } \\
\text { (ordinate) of the single point relative to the normalized maximum } \\
\text { value of unity. }\end{array}$ \\
\hline Occurrence Time & $\begin{array}{l}\text { Sets the time (abscicca) of the single point determining the } \\
\text { exponential decay rate. }\end{array}$ \\
\hline White-Noise Filter Parameters & $\begin{array}{l}\text { The fundamental frequencies and damping ratios of the highpass } \\
\text { and lowpass filters can be adjusted to shape the input frequency } \\
\text { spectrum to the desired frequency content. }\end{array}$ \\
\hline
\end{tabular}




\subsubsection{Ground Motion Generation Parameters Dialog Boxes - Real Spectrum}

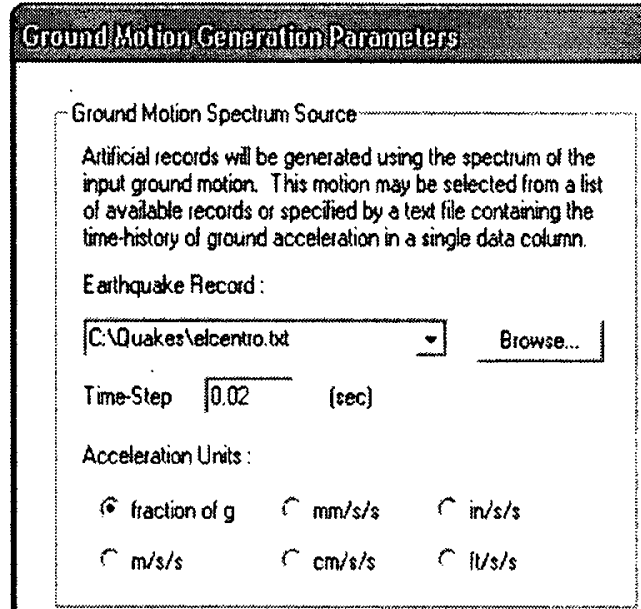

Generalion Parameters

Number of Aulficial Records 100

Number of Saved Recotds 0

C Identical Record Sequence

- Repeatable Record Sequence Seed $\longdiv { 1 2 3 4 5 6 7 }$

C Non-Repealable Record Sequence

Fiter Pecords (Cosine Window)

Low Freq Window Transition 0.00 to $0.01 \quad[\mathrm{~Hz})$

High Freq Window Iransition $\longdiv { 1 0 0 , 0 0 }$ to $100.01 \quad\left(\mathrm{H}_{2}\right)$

〈3ast Cancet $\sqrt{\text { Next }>~}$

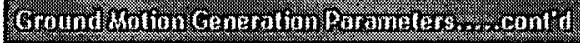

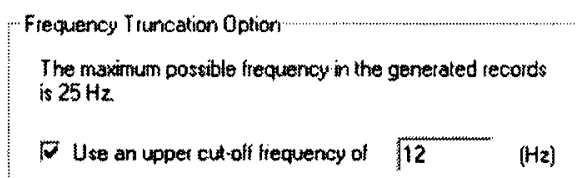

(Hiz)

Duation Truncation Option

The maximum acceleration in the record is $3.12687 \mathrm{~m} / \mathrm{a} / \mathrm{s}$ al $\mathrm{t}=20 \mathrm{~d}$ sea

Truncate ground motion al $\sqrt{113}$ [sec]

¿sok Cancel Nexts

Figure 4.23: Ground Motion Generation Parameters Dialog Boxes - Real Spectrum 


\begin{tabular}{|c|c|}
\hline Field/Option & Notes \\
\hline Earthquake Record & $\begin{array}{l}\text { The input spectral density for generating earthquakes is taken } \\
\text { from the calculated power spectrum of the specified earthquake } \\
\text { record. A deterministic envelope function describing the } \\
\text { temporal amplitude modulation of the record is also calculated } \\
\text { and used for each generated record. The earthquake record } \\
\text { must be stored as a text file containing the time-history data in a } \\
\text { single column. Maximum column width is } 12 \text { characters and } \\
\text { data may be specified in exponential format. }\end{array}$ \\
\hline Time-Step & Time span between earthquake time-history data points. \\
\hline Acceleration Units & Unit system of earthquake acceleration record. \\
\hline Number of Artificial Records & Number of generated earthquakes. \\
\hline Number of Saved Records & $\begin{array}{l}\text { Specifies the number of random earthquake time-histories to be } \\
\text { saved in a text file. Maximum number is } 50 \text {. }\end{array}$ \\
\hline Identical Record Sequence & $\begin{array}{l}\text { The input earthquake time-history is used for each dynamic } \\
\text { analysis. This permits evaluation of the probabilistic response } \\
\text { of structures in which randomness is limited to structural } \\
\text { properties only. }\end{array}$ \\
\hline Repeatable Record Sequence & $\begin{array}{l}\text { For a given random seed value, the same sequence of } \\
\text { earthquake time-histories will be generated each time a multiple } \\
\text { record analysis is done. If structural properties have been } \\
\text { specified to be random, the sequence of random structures is not } \\
\text { repeated. This permits comparison of the relative effect on } \\
\text { probabilistic structural response of earthquake record } \\
\text { randomness vs. various degrees of structural property } \\
\text { randomness. }\end{array}$ \\
\hline Non-Repeatable Record Sequence & $\begin{array}{l}\text { The sequence of generated earthquake time-histories and the } \\
\text { possible sequence of random structural properties are not } \\
\text { repeatable. Specifying this truly random type of multiple record } \\
\text { analysis allows results to be combined with analyses performed } \\
\text { at different times and/or on different computers. }\end{array}$ \\
\hline Filter Records & $\begin{array}{l}\text { This option enables a cosine-type window filter for removal of } \\
\text { high and low frequency content in the earthquake record to } \\
\text { ensure that the ground acceleration record being used for } \\
\text { structural dynamic response analysis is truly representative of a } \\
\text { real earthquake. }\end{array}$ \\
\hline High Frequency Window Transition & $\begin{array}{l}\text { Specifies the frequency range in which the cosine-window } \\
\text { varies from } 1 \text { to } 0 \text {. Frequencies above the upper limit are } \\
\text { removed and frequencies below the lower limit are unaltered. }\end{array}$ \\
\hline Low Frequency Window Transition & $\begin{array}{l}\text { Specifies the frequency range in which the cosine-window } \\
\text { varies from } 0 \text { to } 1 \text {. Frequencies below the lower limit are } \\
\text { removed and frequencies above the upper limit are unaltered. }\end{array}$ \\
\hline Frequency Truncation Option & $\begin{array}{l}\text { This option truncates the calculated power spectrum of the } \\
\text { earthquake at the specified frequency to reduce computation } \\
\text { time in generating earthquake records using the Spectral } \\
\text { Representation method or Filtered Poisson method. This option } \\
\text { is disabled when earthquakes are generated using the inverse } \\
\text { Fast Fourier Transform process. }\end{array}$ \\
\hline Duration Truncation Option & $\begin{array}{l}\text { This option truncates all generated acceleration time-histories at } \\
\text { the specified time to reduce dynamic response computation } \\
\text { time. The occurrence time of maximum acceleration in the } \\
\text { record is provided to aid in setting a truncation time. }\end{array}$ \\
\hline
\end{tabular}




\subsubsection{Elastic Responses Dialog Box}

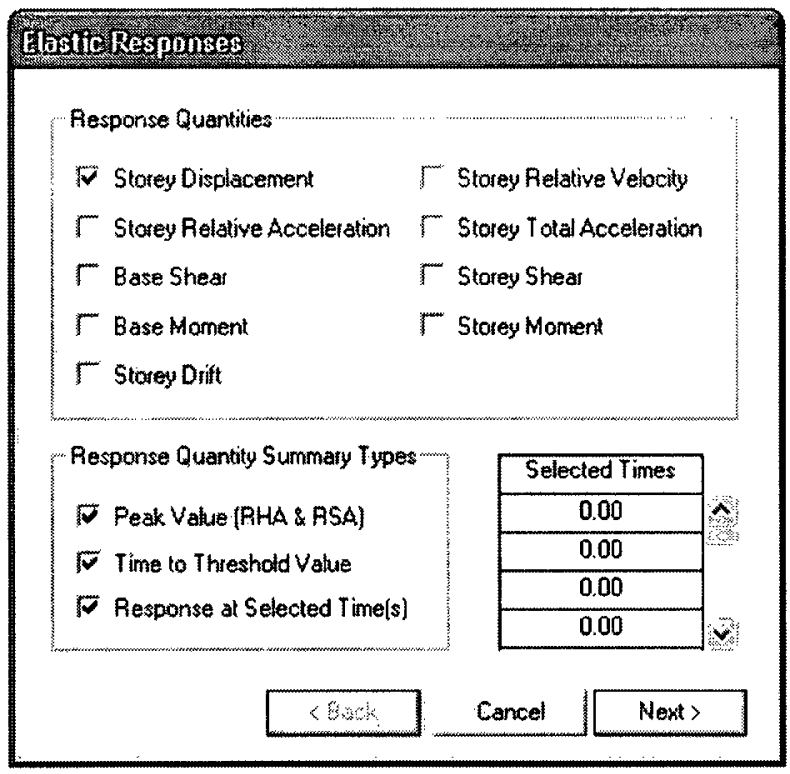

Figure 4.24: Elastic Responses Dialog Box

\begin{tabular}{|c|l|}
\hline Field/Option & \multicolumn{1}{|c|}{ Notes } \\
\hline Response Quantities & $\begin{array}{l}\text { Any combination of response quantities may be selected. Each } \\
\text { selection may have a different combination of specified storeys. Once } \\
\text { all elastic response quantities have been selected, a Storey Selection } \\
\text { Dialog Box will appear for each response quantity. See Figure 4.25. }\end{array}$ \\
\hline Peak Value & $\begin{array}{l}\text { This option is enabled for multiple record analysis only. The peak } \\
\text { value of each selected elastic response quantity for each selected storey } \\
\text { for each earthquake time-history analysis will be included in an output } \\
\text { file. See Figure 4.45. Peak values are determined from the response } \\
\text { history analysis (RHA) as well as a response spectrum analysis (RSA), } \\
\text { which combines maximum modal values using the absolute sum } \\
\text { (ABSSUM), square-root-of-sum-of-squares (SRSS) and complete } \\
\text { quadratic combination (CQC) modal combination rules. }\end{array}$ \\
\hline Time to Threshold Value & $\begin{array}{l}\text { This option is enabled for multiple record analysis only. Selecting this } \\
\text { option allows a threshold value for each selected response quantity to } \\
\text { be specified for each selected storey in the Storey Selection Dialog } \\
\text { Box. See Figure 4.25. The time of the first crossing of the specified } \\
\text { threshold is recorded for each earthquake response analysis. If the } \\
\text { threshold is not reached, the threshold time is recorded as 0.0. The } \\
\text { recorded times are included in an output file. See Figure 4.45. }\end{array}$ \\
\hline Response at Selected Time(s) \\
$\begin{array}{l}\text { This option is enabled for multiple record analysis only. Selecting this } \\
\text { option turns on a scroll box where a maximum of 20 response times } \\
\text { may be entered. The value of each selected response quantity for each } \\
\text { selected storey at the specified times will be recorded. The recorded } \\
\text { values are included in an output file. See Figure 4.45. }\end{array}$ \\
\hline
\end{tabular}




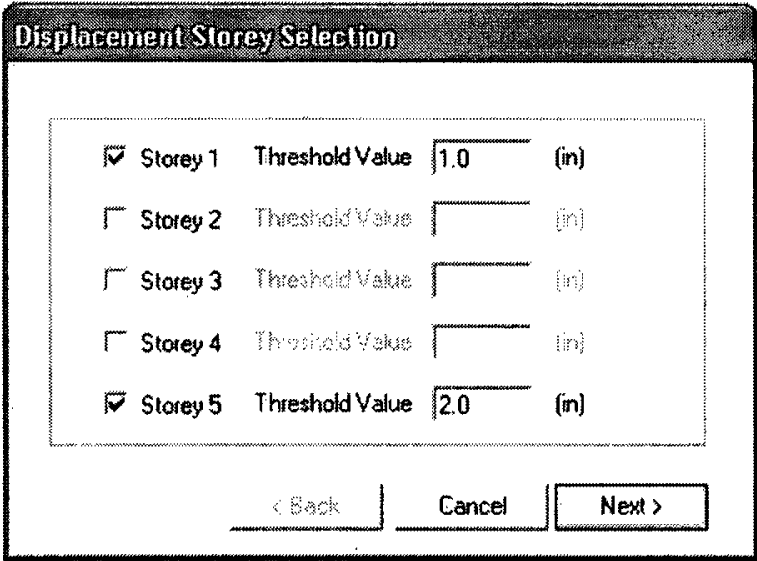

Figure 4.25: Storey Selection Example Dialog Box

\subsubsection{SDOF Inelastic Responses Dialog Box}

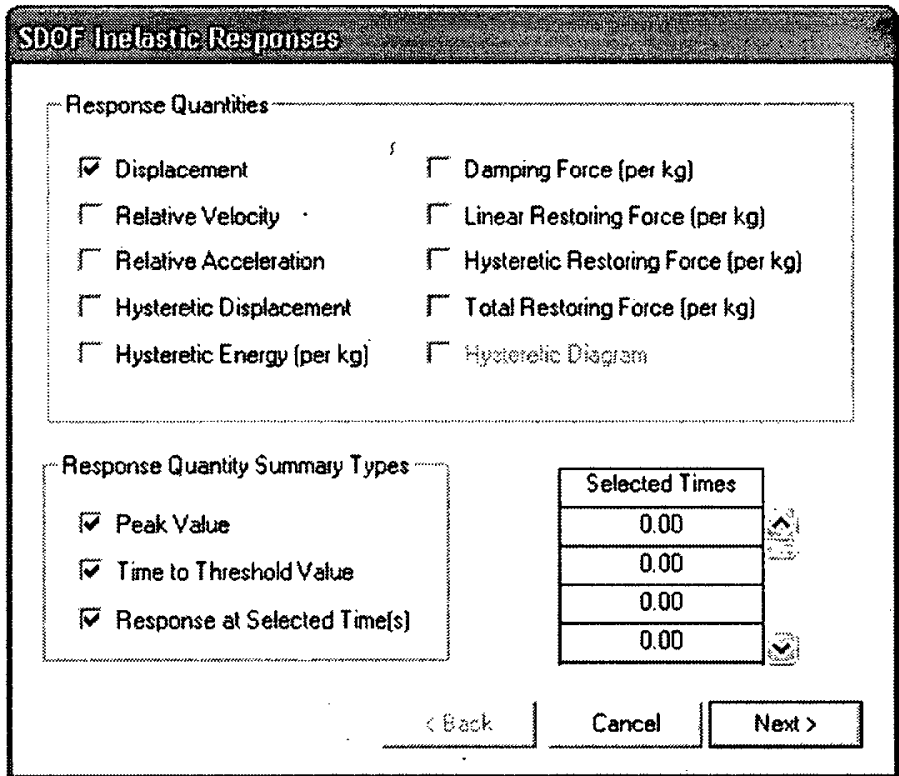

Figure 4.26: SDOF Inelastic Responses Dialog Box

\begin{tabular}{|c|l|}
\hline Field/Option & \multicolumn{1}{c|}{ Notes } \\
\hline Response Quantities & $\begin{array}{l}\text { Any combination of response quantities may be selected. The force and energy } \\
\text { response quantities are expressed per unit mass since a single-degree-of- } \\
\text { freedom structure may be specified by natural frequency only, in which case } \\
\text { mass is not known. The response quantities are defined as follows: } \\
\text { Displacement }: \text { lateral displacement of lumped mass with respect to the ground. }\end{array}$ \\
\hline
\end{tabular}




\begin{tabular}{|c|c|}
\hline & $\begin{array}{l}\text { Hysteretic Displacement : displacement } z \text { in Equation [3.4]. } \\
\text { Relative Velocity: velocity of lumped mass with respect to the ground. } \\
\text { Relative Acceleration : acceleration of lumped mass with respect to the ground. } \\
\text { Damping Force : product of damping and relative velocity. } \\
\text { Linear Restoring Force : product of initial stiffness per unit mass, displacement } \\
\text { and post-yield stiffness. See Equation [3.4]. } \\
\text { Hysteretic Restoring Force : product of initial stiffness per unit mass, hysteretic } \\
\text { displacement and (1-post-yield stiffness). See Equation [3.4]. } \\
\text { Total Restoring Force : sum of linear restoring force and hysteretic restoring } \\
\text { force. } \\
\text { Hysteretic Energy : Summed product of hysteretic restoring force and } \\
\text { displacement. } \\
\text { Hysteretic Diagram : this option enables an onscreen plot of the response } \\
\text { hysteresis loop for a single earthquake analysis. This option is disabled for } \\
\text { multiple record analysis. }\end{array}$ \\
\hline Peak Value & $\begin{array}{l}\text { See Section 4.4.14. Since the dynamic structural response to each earthquake } \\
\text { time-history is assumed to be inelastic, the peak value of each selected elastic } \\
\text { response quantity for each selected storey is determined from the response } \\
\text { history analysis (RHA) only. Response spectrum analysis does not apply. }\end{array}$ \\
\hline $\begin{array}{c}\text { Time to Threshold } \\
\text { Value }\end{array}$ & $\begin{array}{l}\text { See Section } 4.4 .14 \text {. Although an SDOF structure is by definition a single storey } \\
\text { structure, when this option is selected a Storey Selection Dialog Box will appear } \\
\text { for each selected response quantity to permit threshold values to be specified. } \\
\text { See Figure } 4.25 \text {. }\end{array}$ \\
\hline $\begin{array}{c}\text { Response at Selected } \\
\text { Time(s) }\end{array}$ & See Section 4.4.14. \\
\hline
\end{tabular}

\subsubsection{MDOF Inelastic Responses Dialog Box}

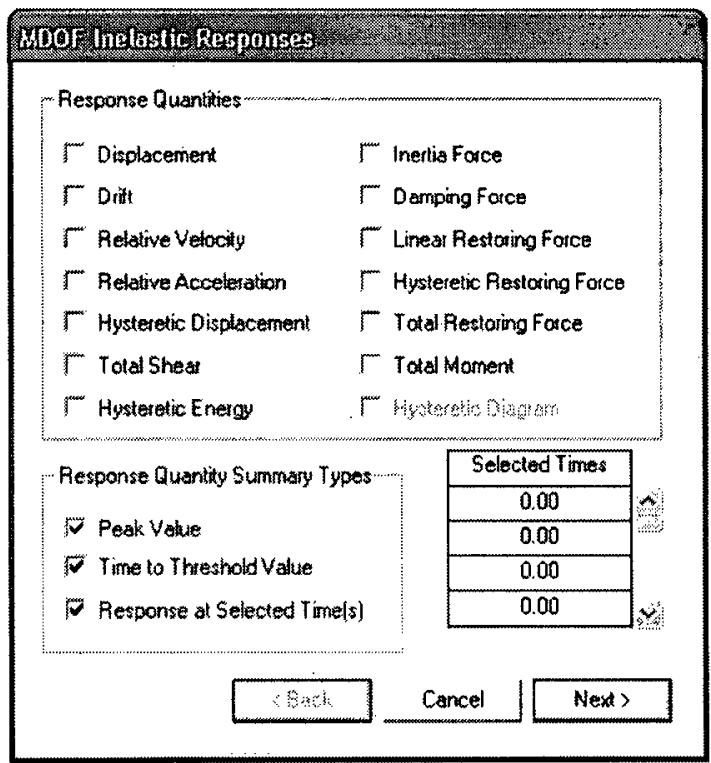

Figure 4.27: MDOF Inelastic Responses Dialog Box 


\begin{tabular}{|c|l|}
\hline Field/Option & \multicolumn{1}{c|}{ Notes } \\
\hline Response Quantities & $\begin{array}{l}\text { See Section } 4.4 .15 \text { for definitions of response quantities. For } \\
\text { multiple-degree-of-freedom structures, with known storey masses, } \\
\text { the force and energy response quantities are expressed as total force } \\
\text { and total energy. }\end{array}$ \\
\hline Peak Value & See Section 4.4.15. \\
\hline Time to Threshold Value & See Section 4.4.14. \\
\hline Response at Selected Time(s) & See Section 4.4.14. \\
\hline
\end{tabular}

\subsubsection{Plot Modal Results Dialog Box}

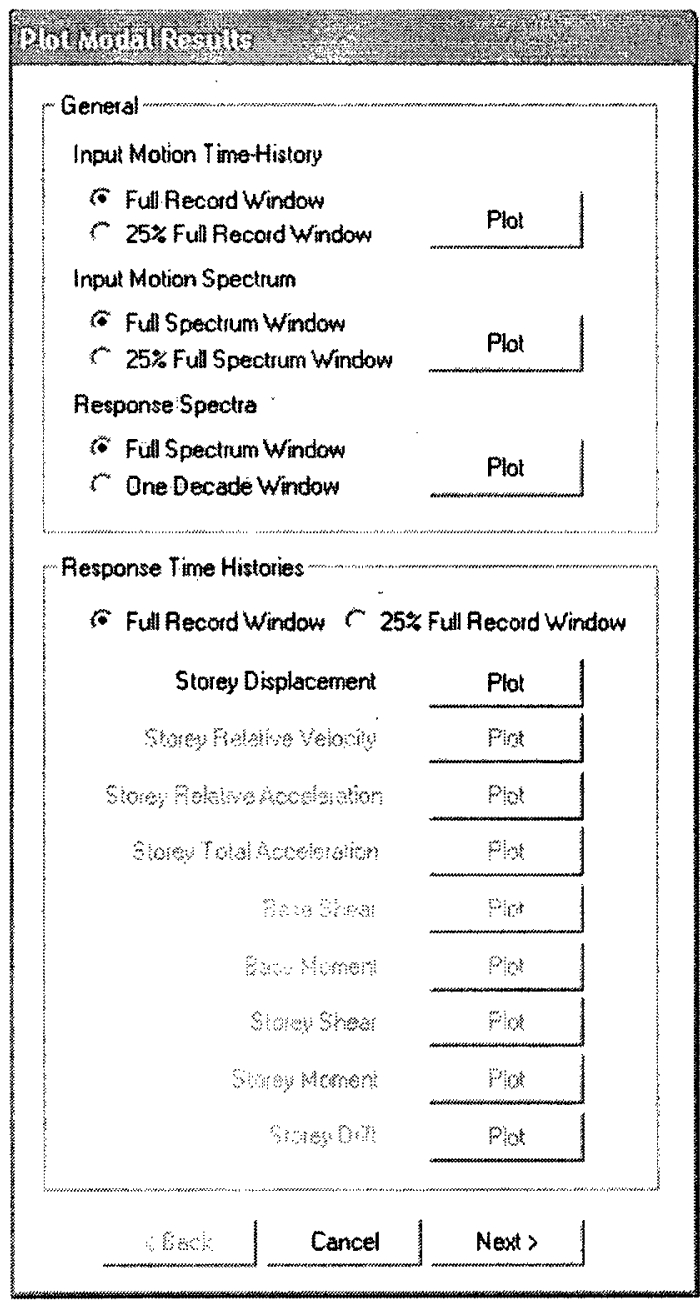

Figure 4.28: Plot Modal Results Dialog Box 


\begin{tabular}{|c|l|}
\hline Button/Option & \multicolumn{1}{|c|}{ Notes } \\
\hline \multirow{2}{*}{$\begin{array}{c}\text { Input Motion } \\
\text { Time-History }\end{array}$} & $\begin{array}{l}\text { The Plot Modal Results dialog box is displayed only if the Oscillation Response } \\
\text { option or Single Earthquake Response option was chosen along with the Elastic } \\
\text { Analysis option from the initial General Input Parameters dialog box. This } \\
\text { gutton activates a pop-up dialog box that displays the time-history of the input } \\
\text { Record Window option displays the time-history in a scale that fits the futire } \\
\text { record across the width of the pop-up dialog box. For a more detailed display, } \\
\text { select the expanded scale given by the 25\% Full Record Window option. Once } \\
\text { the pop-up dialog box has been displayed, it may be closed and re-opened } \\
\text { repeatedly with either time-scale. See Figure 4.29. }\end{array}$ \\
\hline Input Motion & $\begin{array}{l}\text { Displays the power spectrum of the input earthquake motion. The power scale is } \\
\text { normalized to the maximum power value in the record. This button is disabled if } \\
\text { the Oscillation Response option was selected in the General Input Parameters } \\
\text { dialog box. See Figure 4.30. }\end{array}$ \\
\hline Response Spectra & $\begin{array}{l}\text { Displays the response spectra for displacement, pseudo-velocity, pseudo- } \\
\text { acceleration, ratio of pseudo-velocity to relative velocity and ratio of pseudo- } \\
\text { acceleration to relative acceleration for each damping level specified previously. } \\
\text { This button is disabled if the Calculate Response Spectra option was not checked } \\
\text { in the Single Ground Motion Parameters dialog box. See Figure 4.31. }\end{array}$ \\
\hline Response Time- & $\begin{array}{l}\text { Displays a separate pop-up dialog box for each storey that was selected for a } \\
\text { given response quantity. The pop-up dialog box displays the individual modal } \\
\text { Histories } \\
\text { responses as well as the total response. See Figure 4.32. }\end{array}$ \\
\hline
\end{tabular}

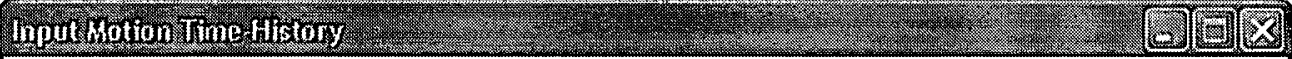
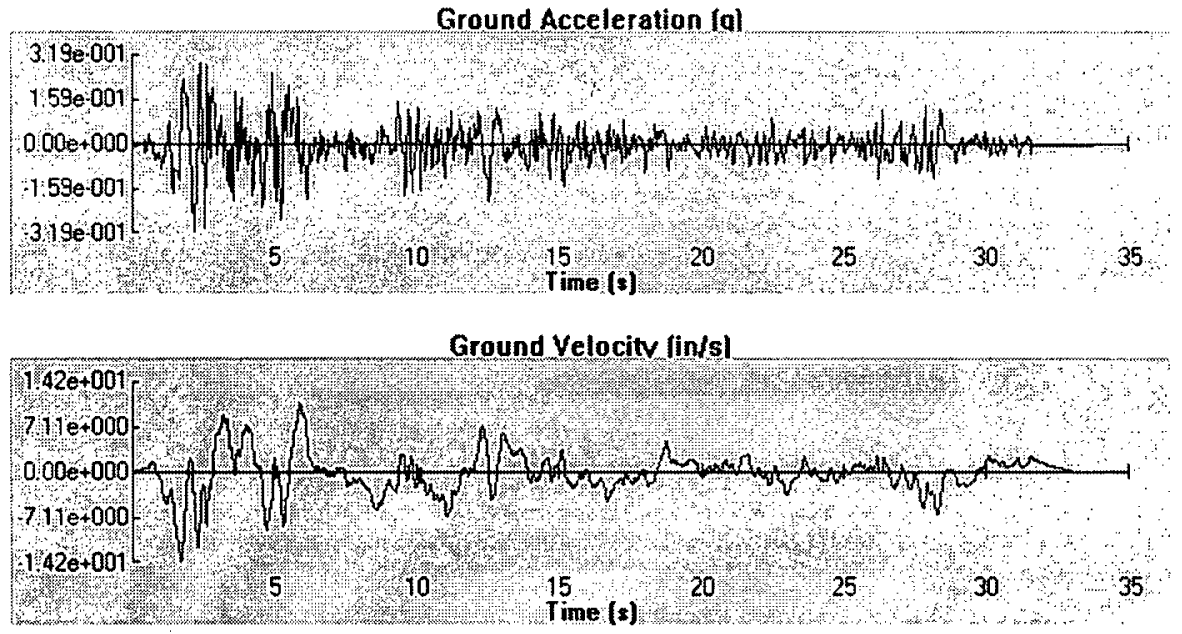

Ground Displacement finl

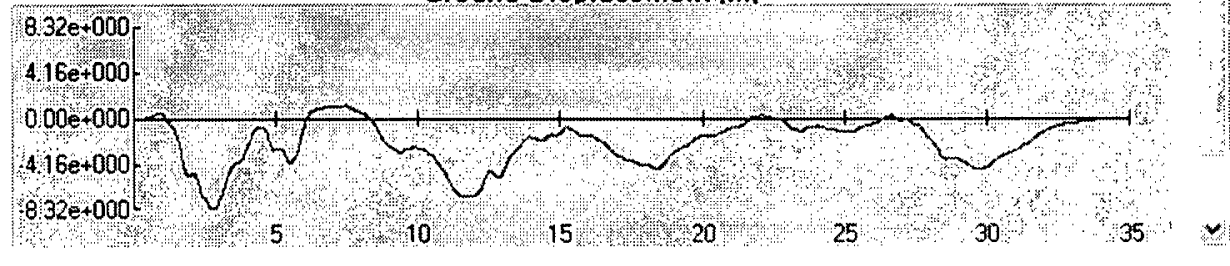

Figure 4.29: Input Motion Time-History Pop-Up Dialog Box 


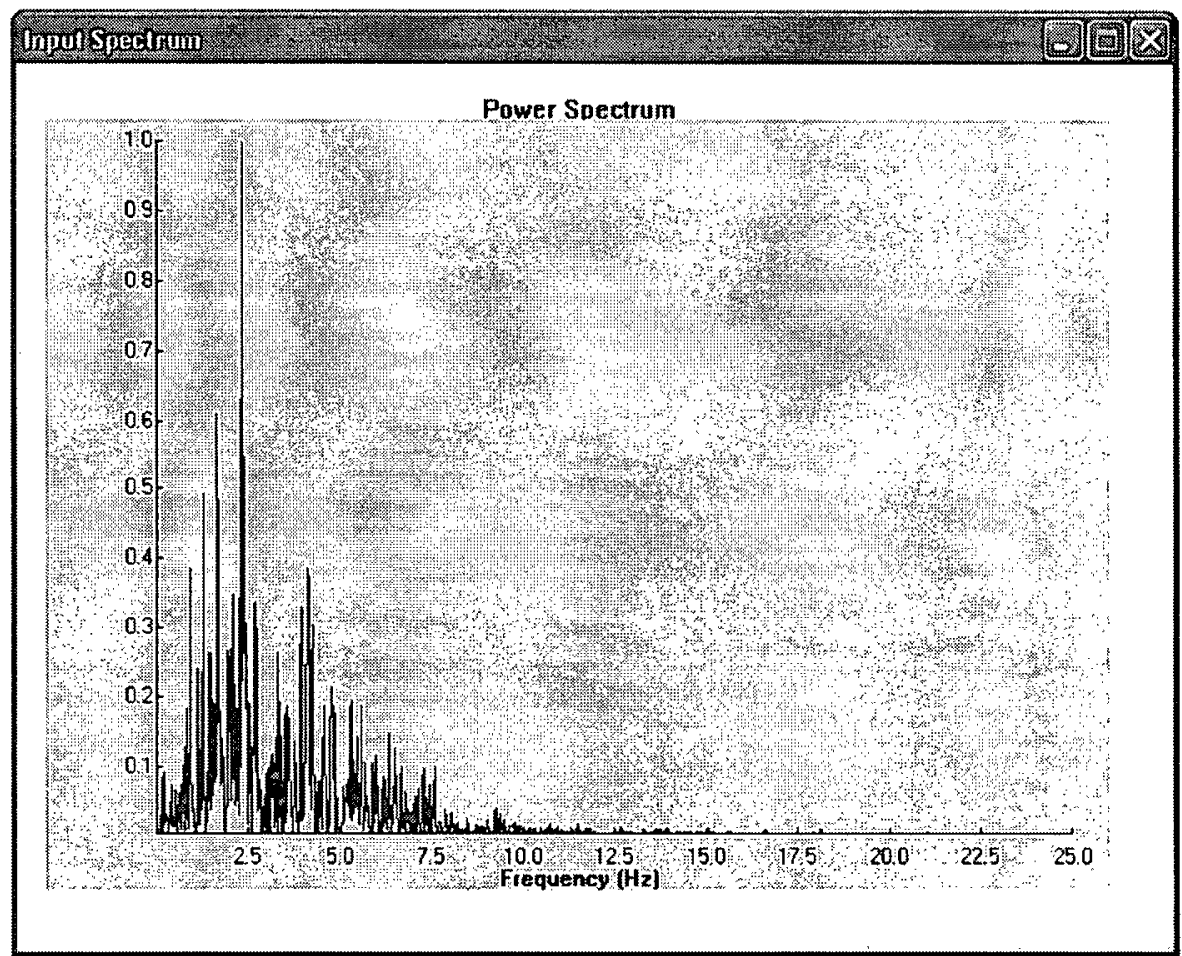

Figure 4.30: Input Spectrum Pop-Up Dialog Box

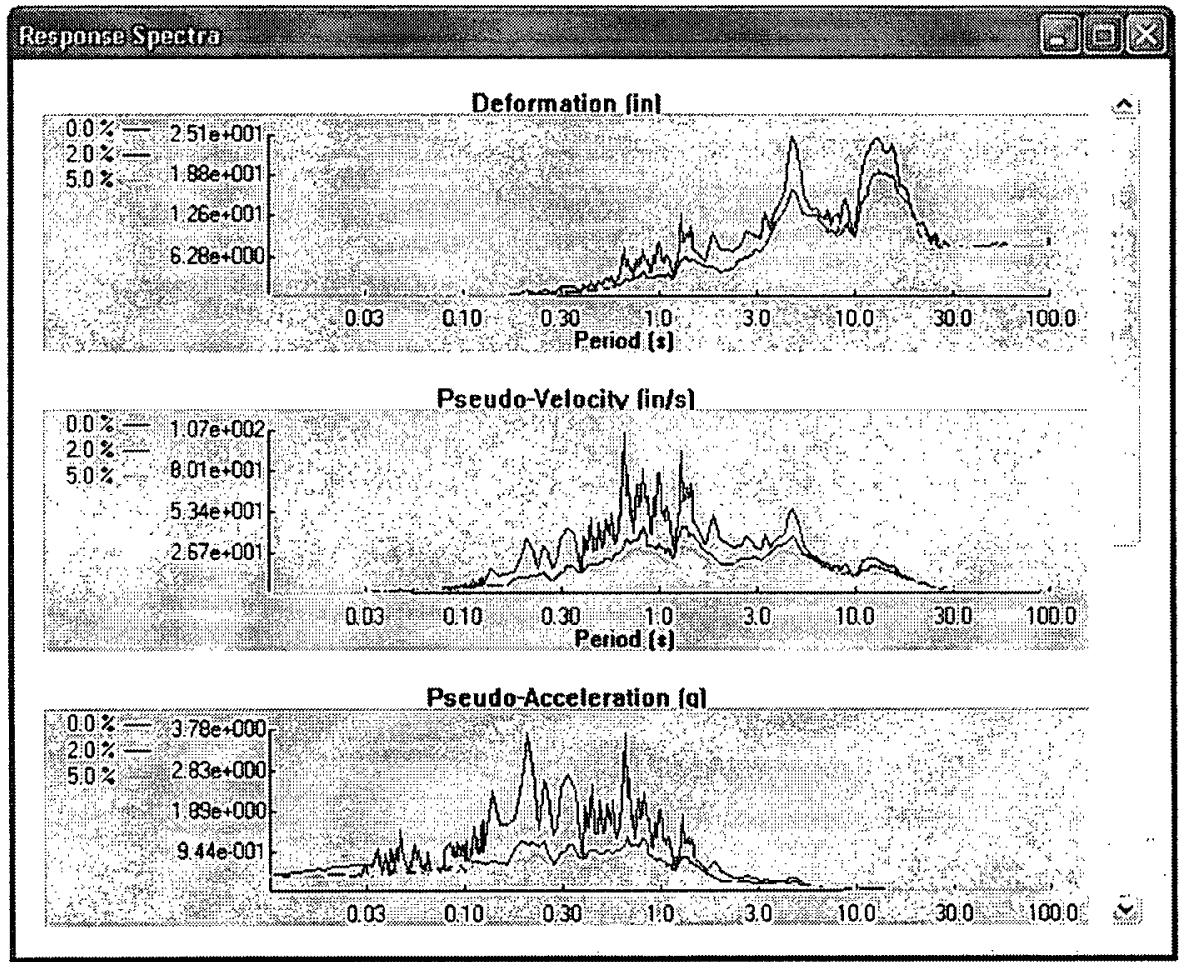

Figure 4.31: Response Spectra Pop-Up Dialog Box 


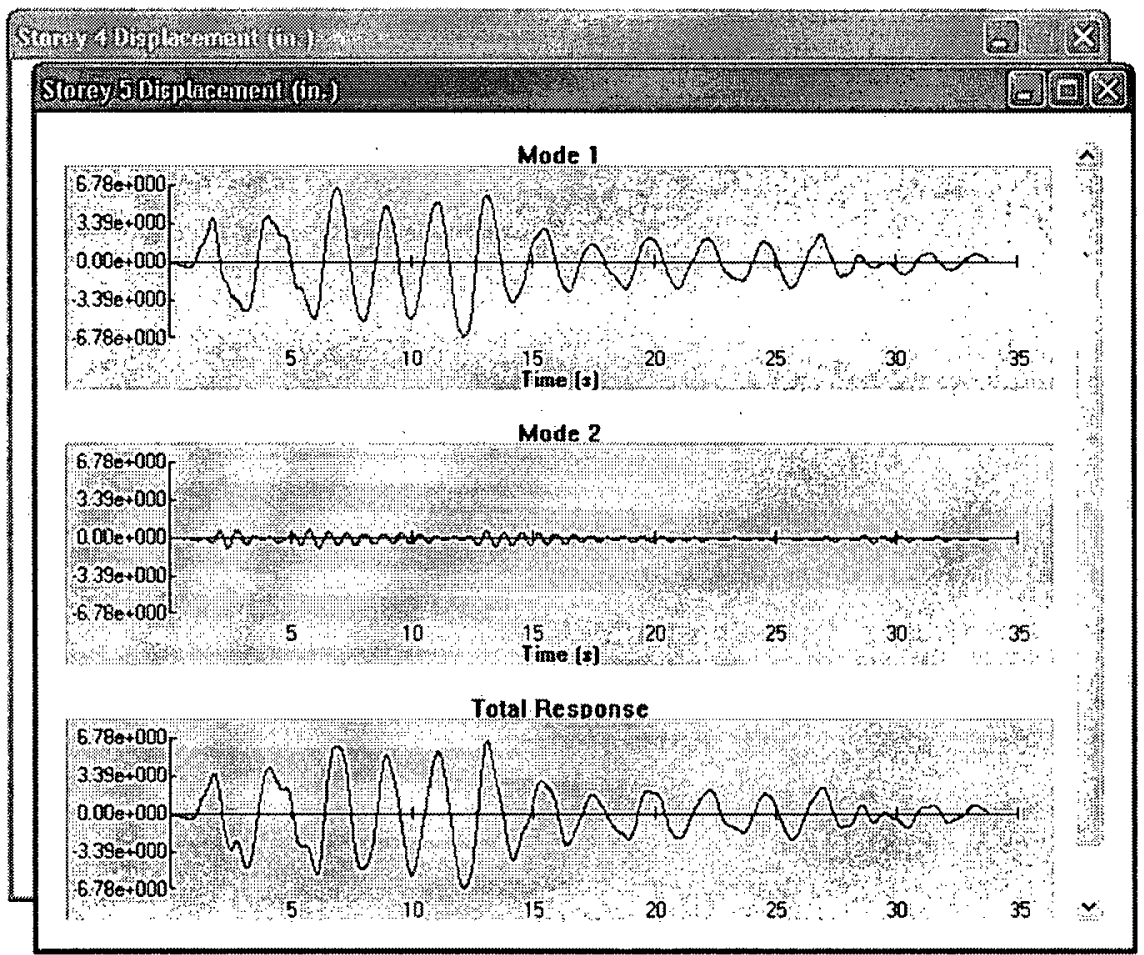

Figure 4.32: Modal Response Time-History Example Pop-Up Dialog Box

\subsubsection{Plot SDOF Results Dialog Box}

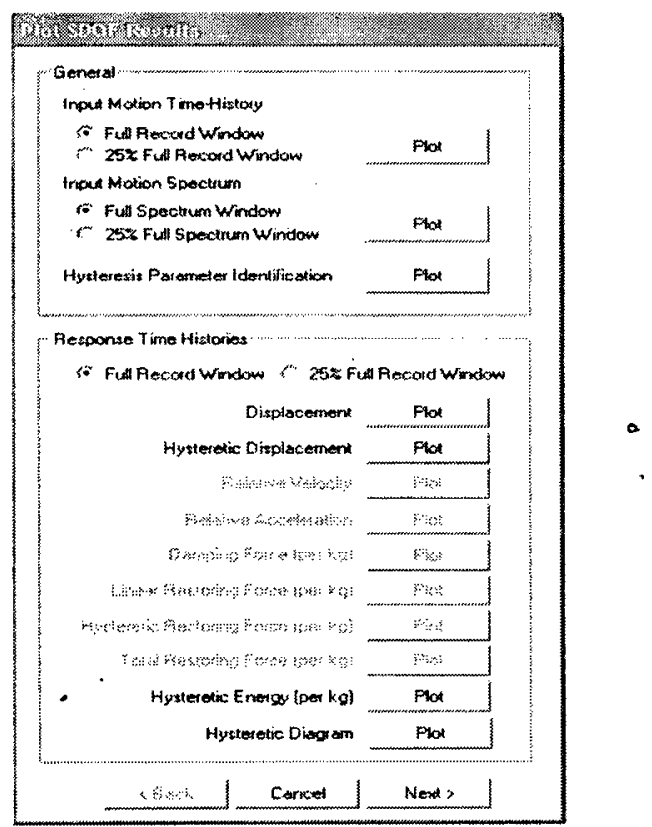

Figure 4.33: Plot SDOF Results Dialog Box 


\begin{tabular}{|c|l|}
\hline Button/Option & \multicolumn{1}{|c|}{ Notes } \\
\hline Input Motion Time-History & $\begin{array}{l}\text { The Plot SDOF Results dialog box is displayed only if the Oscillation } \\
\text { Response option or Single Earthquake Response option was chosen } \\
\text { along with the Inelastic Analysis option from the initial General } \\
\text { Input Parameters dialog box. See Section 4.4.17. }\end{array}$ \\
\hline Input Motion Spectrum & See Section 4.4.17. \\
\hline \multirow{5}{*}{$\begin{array}{l}\text { Hysteresis Parameter } \\
\text { Identification }\end{array}$} & $\begin{array}{l}\text { Displays a comparison of the input hysteresis data with the model } \\
\text { hysteretic response for the same displacement or acceleration time- } \\
\text { history. If the Quasi-Static Force vs. Displacement option was } \\
\text { specified for input hysteresis data, only the input and model } \\
\text { hysteresis loops are displayed. If the Acceleration Response option } \\
\text { was specified for input hysteresis data, the input and model } \\
\text { acceleration response time-histories are displayed along with the } \\
\text { associated model hysteresis loop. The Hysteresis Parameter } \\
\text { Identification button is disabled if the Use Default Hysteresis } \\
\text { Parameters option was selected in the initial Hysteresis Parameter } \\
\text { Identification dialog box. See Figure 4.34 and Figure 4.35. }\end{array}$ \\
\hline Response Time-Histories & $\begin{array}{l}\text { Displays the response time-history for the selected response quantity. } \\
\text { See Section 4.4.19. }\end{array}$ \\
\hline
\end{tabular}

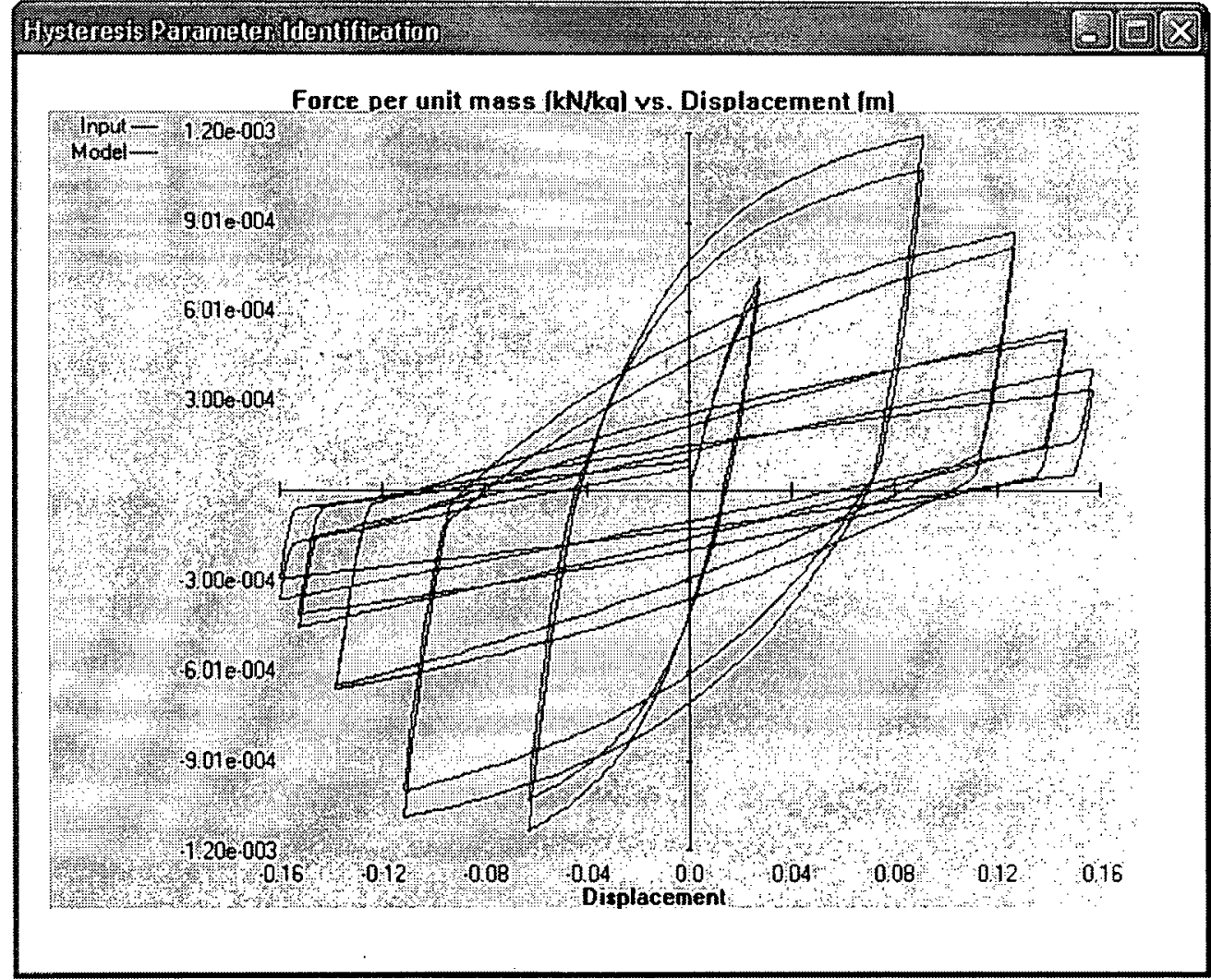

Figure 4.34: Hysteresis Parameter Identification Dialog Box (Quasi-Static Data) 


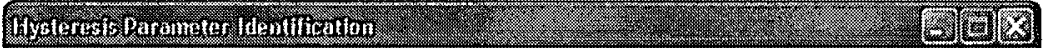
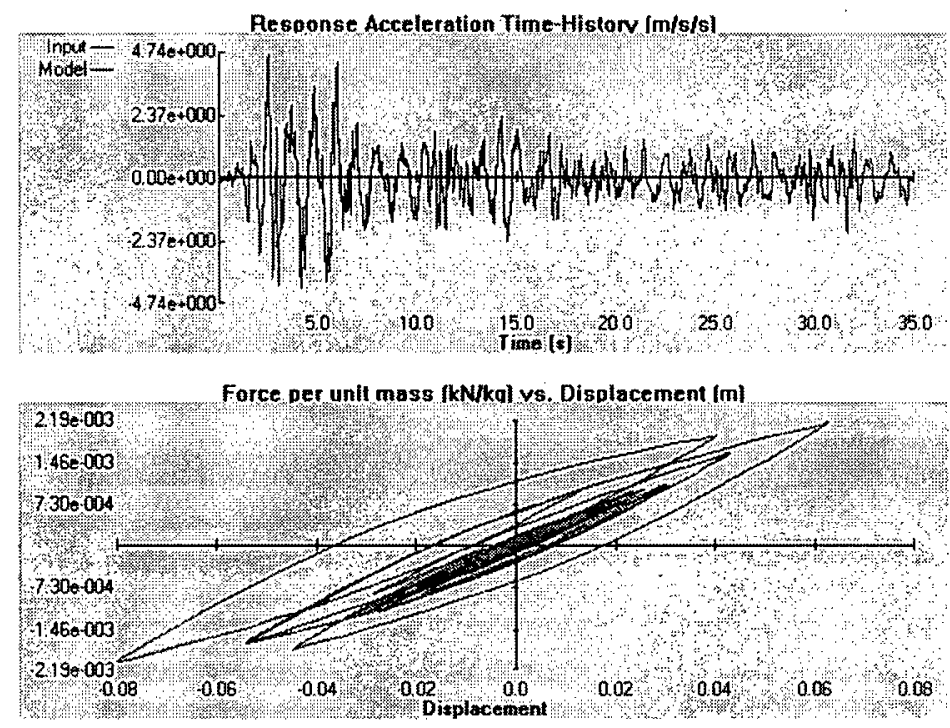

Figure 4.35: Hysteresis Parameter Identification Dialog Box (Acceleration Data)

\subsubsection{Plot MDOF Results Dialog Box}

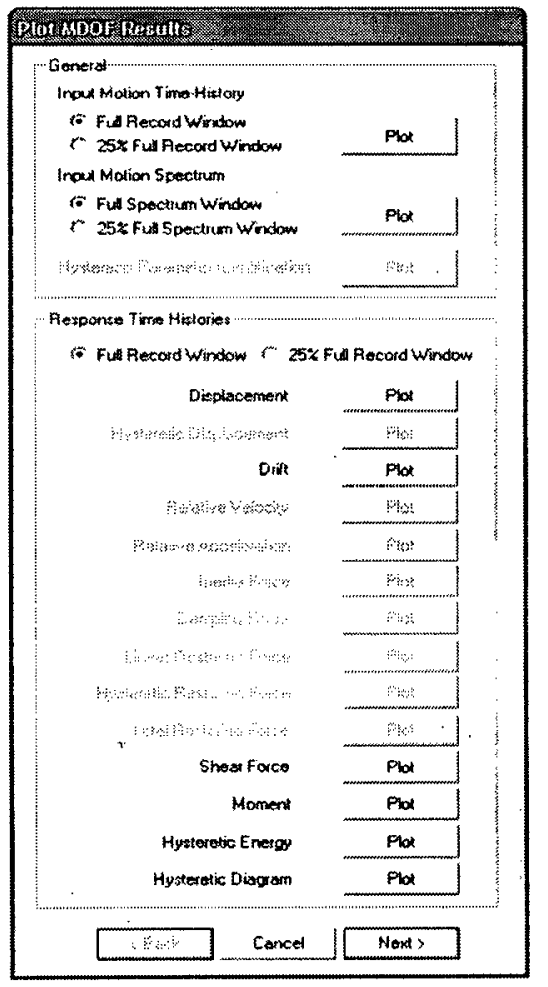

Figure 4.36: Plot MDOF Results Dialog Box 


\begin{tabular}{|c|l|}
\hline Button/Option & \multicolumn{1}{|c|}{ Notes } \\
\hline Input Motion Time-History & $\begin{array}{l}\text { The Plot MDOF Results dialog box is displayed only if the } \\
\text { Oscillation Response option or Single Earthquake Response } \\
\text { option was chosen along with the Inelastic Analysis option } \\
\text { from the initial General Input Parameters dialog box. See } \\
\text { Section 4.4.17. }\end{array}$ \\
\hline Input Motion Spectrum & See Section 4.4.17. \\
\hline Hysteresis Parameter Identification & See Section 4.4.18. \\
\hline Response Time-Histories & $\begin{array}{l}\text { Displays the response time-history of each selected storey } \\
\text { for a given response quantity on a single pop-up dialog box. } \\
\text { See Figure 4.37, Figure 4.38 and Figure 4.39. }\end{array}$ \\
\hline
\end{tabular}

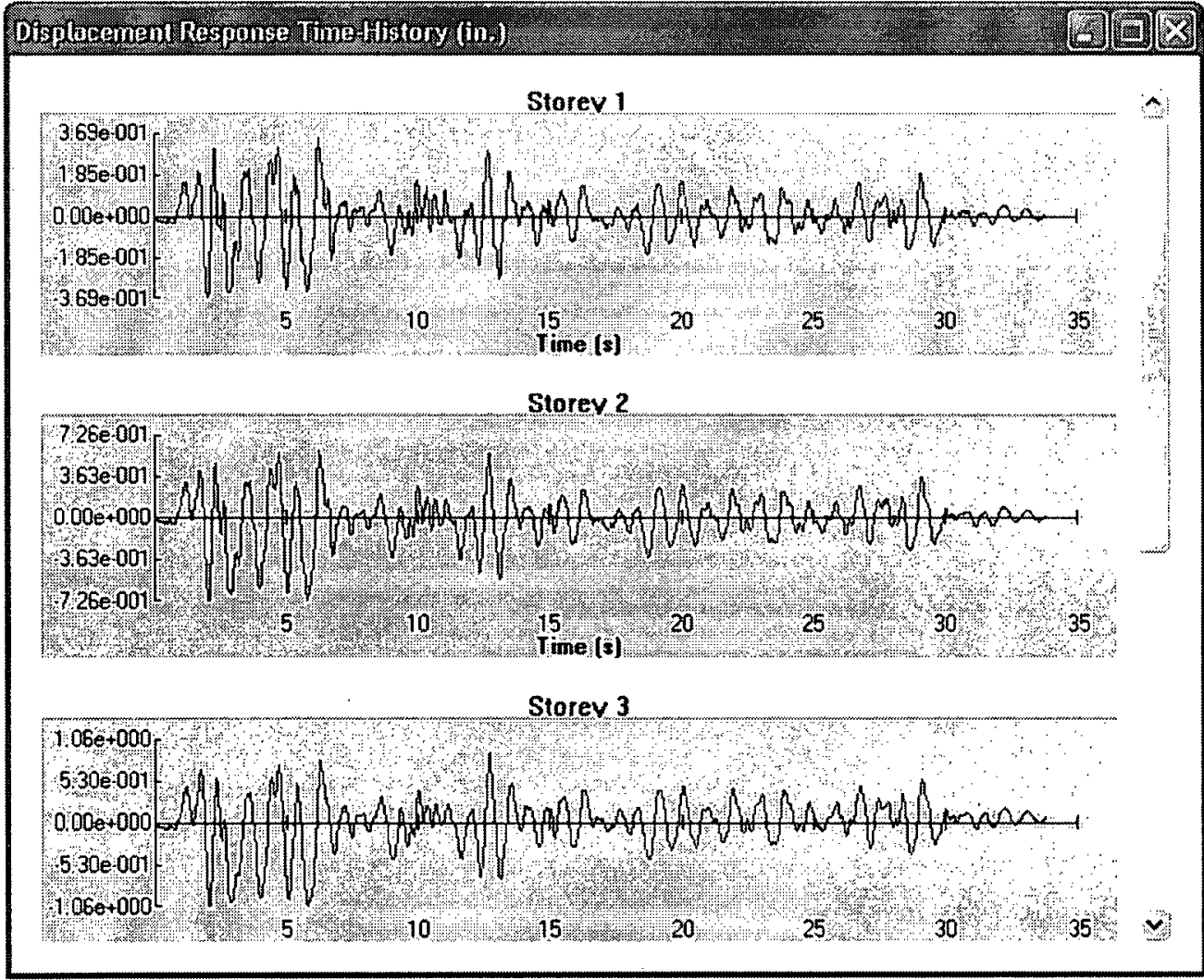

Figure 4.37: MDOF Response Time-History Example Pop-Up Dialog Box (Displacement) 


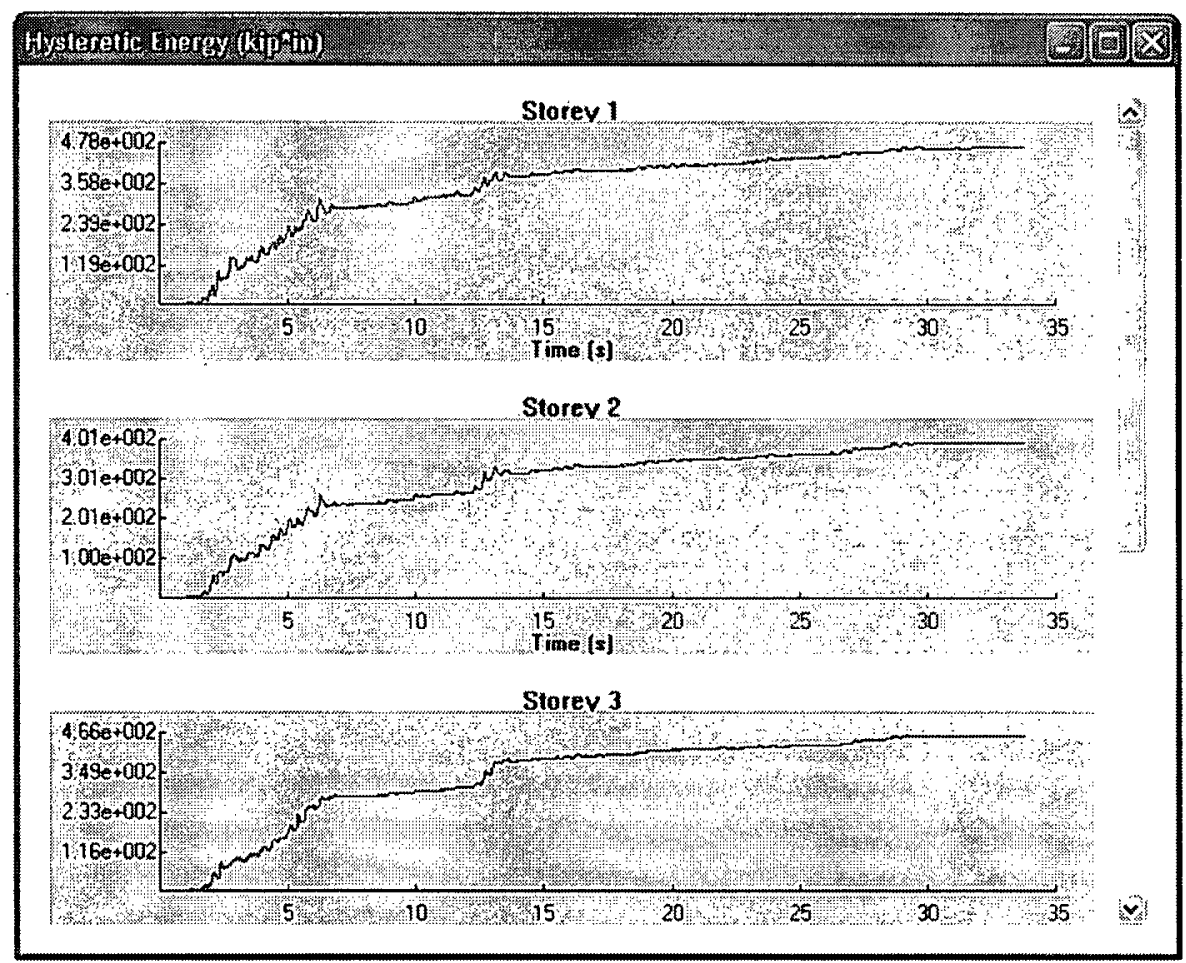

Figure 4.38: MDOF Response Time-History Example Pop-Up Dialog Box (Energy)

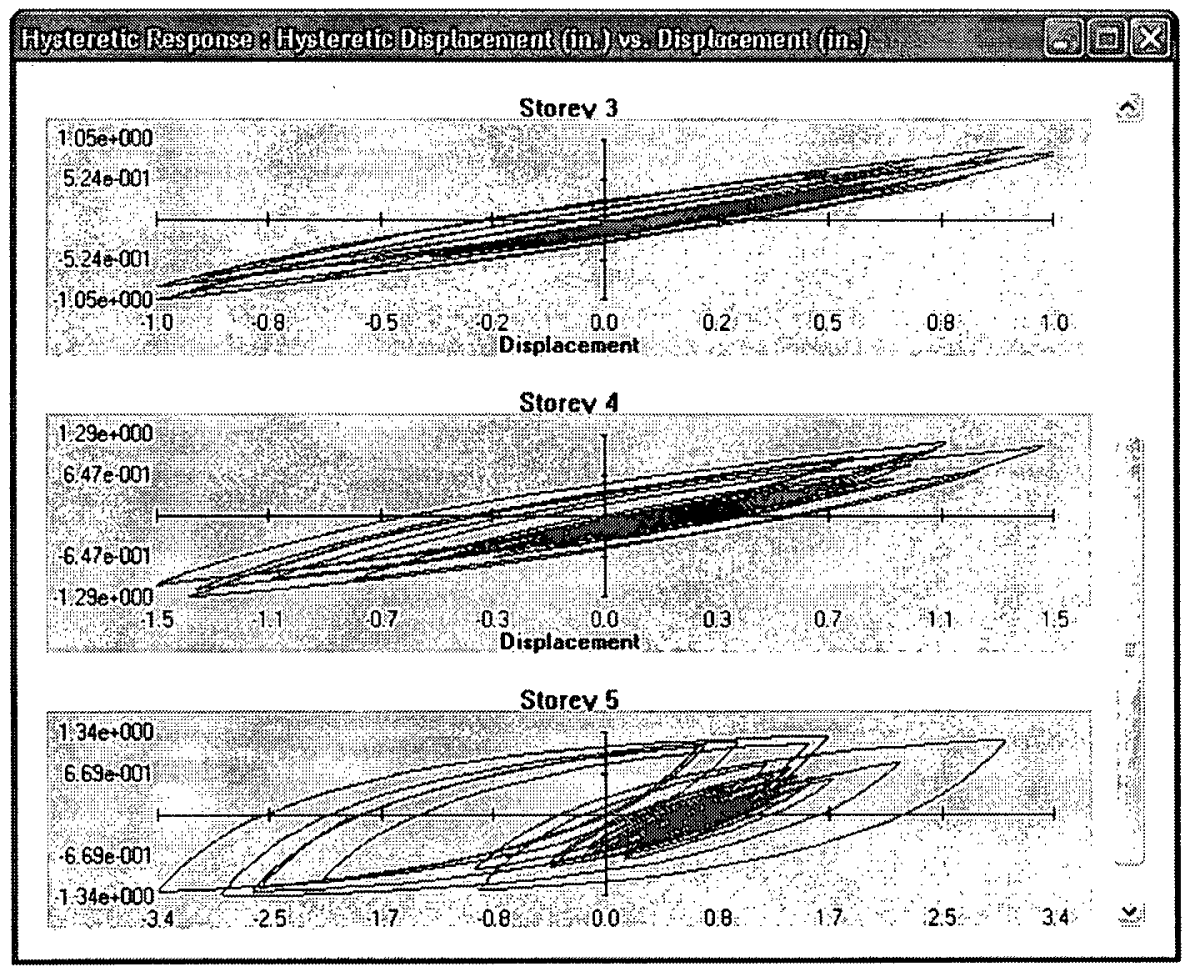

Figure 4.39: MDOF Response Time-History Example Pop-Up Dialog Box (Hysteresis) 


\subsubsection{Print Results Dialog Box}

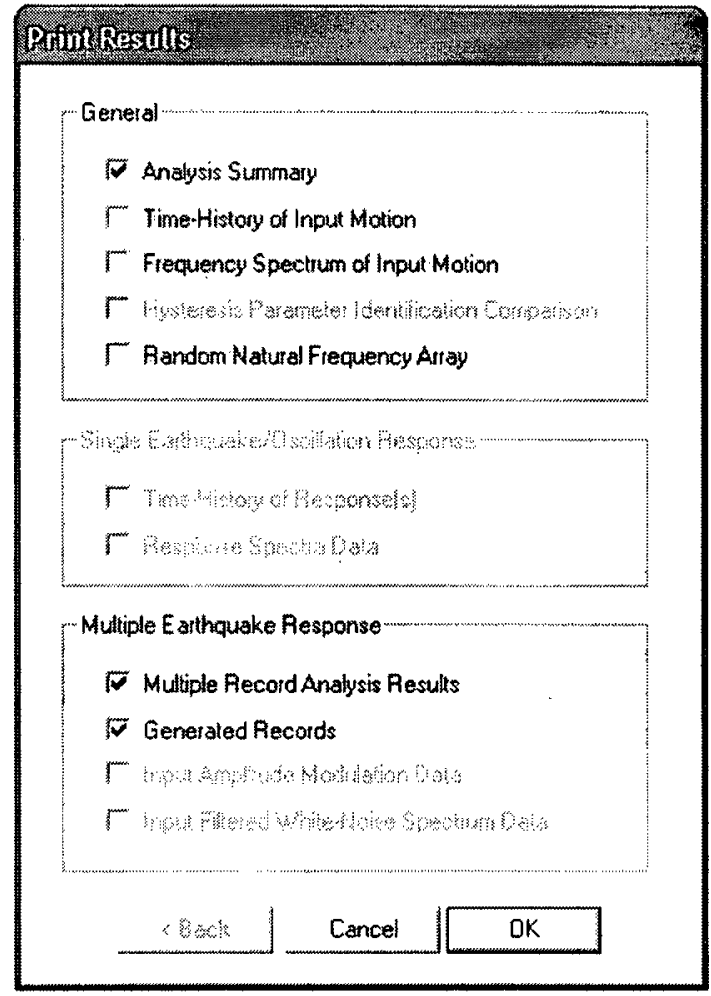

Figure 4.40: Print Results Dialog Box

\begin{tabular}{|c|c|}
\hline Option & Notes \\
\hline Analysis Summary & Summarizes all input data. See Figure 4.41 . \\
\hline Time-History of Input Motion & $\begin{array}{l}\text { Base motion acceleration, velocity and displacement time- } \\
\text { history data. }\end{array}$ \\
\hline Frequency Spectrum of Input Motion & $\begin{array}{l}\text { Calculated power spectrum data from input earthquake } \\
\text { motion. This option is disabled if Oscillation Response } \\
\text { was selected in the General Input Parameters dialog box. }\end{array}$ \\
\hline $\begin{array}{c}\text { Hysteresis Parameter Identification } \\
\text { Comparison }\end{array}$ & $\begin{array}{l}\text { Hysteresis model comparison data used in the onscreen } \\
\text { plots of Section } 4.4 .18 \text { and Section } 4.4 .19 \text {. This option is } \\
\text { disabled if the Elastic Analysis option was selected in the } \\
\text { General Input Parameters dialog box or the Use Default } \\
\text { Hysteresis Parameters option was selected in the initial } \\
\text { Hysteresis Parameter Identification dialog box. }\end{array}$ \\
\hline Random Natural Frequency Array & $\begin{array}{l}\text { Calculated modal frequencies for each random structure } \\
\text { generated in a multiple record analysis. This option is } \\
\text { disabled if the Oscillation Response option or Single } \\
\text { Earthquake Response option was selected in the General } \\
\text { Input Parameters dialog box or structural properties were } \\
\text { specified as deterministic in the Multiple Earthquake } \\
\text { Analysis Parameters dialog box. See Figure } 4.42 \text {. }\end{array}$ \\
\hline $\bar{T}$ & Time-history data used in the onscreen plots of Section \\
\hline
\end{tabular}




\begin{tabular}{|c|c|}
\hline & $\begin{array}{l}\text { 4.4.17, Section } 4.4 .18 \text { and Section } 4.4 .19 \text {. If the Elastic } \\
\text { Analysis option was selected in the General Input } \\
\text { Parameters dialog box, the data is saved in a separate text } \\
\text { file for each selected storey of each selected response } \\
\text { quantity. See Figure } 4.43 \text {. If the Inelastic Analysis option } \\
\text { was selected, the data for each selected storey of a given } \\
\text { response quantity is saved in one text file. This Time- } \\
\text { History of Response(s) option is disabled if the Multiple } \\
\text { Earthquake Response option was selected in the General } \\
\text { Input Parameters dialog box. }\end{array}$ \\
\hline Response Spectra Data & $\begin{array}{l}\text { Response spectra data used in the onscreen plots of } \\
\text { Section 4.4.17. This option is disabled if the Multiple } \\
\text { Earthquake Response option or the Inelastic Analysis } \\
\text { option was selected in the General Input Parameters } \\
\text { dialog box or the Calculate Response Spectra option was } \\
\text { not checked in the Single Ground Motion Parameters } \\
\text { dialog box. See Figure } 4.44 \text {. }\end{array}$ \\
\hline Multiple Record Analysis Results & $\begin{array}{l}\text { Peak values, threshold times and response values at } \\
\text { selected times for each selected response quantity for each } \\
\text { earthquake time-history analysis. This option is disabled if } \\
\text { the Oscillation Response option or Single Earthquake } \\
\text { Response option was selected in the General Input } \\
\text { Parameters dialog box. See Figure } 4.45 \text {. }\end{array}$ \\
\hline Generated Records & $\begin{array}{l}\text { Time-history acceleration data and frequency spectrum } \\
\text { data for the specified number of saved artificial earthquake } \\
\text { records. This option is disabled if the Oscillation } \\
\text { Response option or Single Earthquake Response option } \\
\text { was selected in the General Input Parameters dialog box } \\
\text { or the number of saved records was set at zero in the } \\
\text { Ground Motion Generation Parameters dialog box. }\end{array}$ \\
\hline Input Amplitude Modulation Data & $\begin{array}{l}\text { Temporal amplitude modulation data corresponding to the } \\
\text { onscreen plot in the Ground Motion Generation } \\
\text { Parameters(Filtered Spectrum) dialog box. This option is } \\
\text { disabled if the Oscillation Response option or Single } \\
\text { Earthquake Response option was selected in the General } \\
\text { Input Parameters dialog box or the Earthquake Record } \\
\text { input spectrum option was selected in the Multiple } \\
\text { Earthquake Record Analysis Parameters dialog box. }\end{array}$ \\
\hline Input Filtered White-Noise Spectrum Data & $\begin{array}{l}\text { Input frequency spectrum data corresponding to the } \\
\text { onscreen plot in the Ground Motion Generation } \\
\text { Parameters(Filtered Spectrum) dialog box. This option is } \\
\text { disabled if the Oscillation Response option or Single } \\
\text { Earthquake Response option was selected in the General } \\
\text { Input Parameters dialog box or the Earthquake Record } \\
\text { input spectrum option was selected in the Multiple } \\
\text { Earthquake Record Analysis Parameters dialog box. }\end{array}$ \\
\hline
\end{tabular}




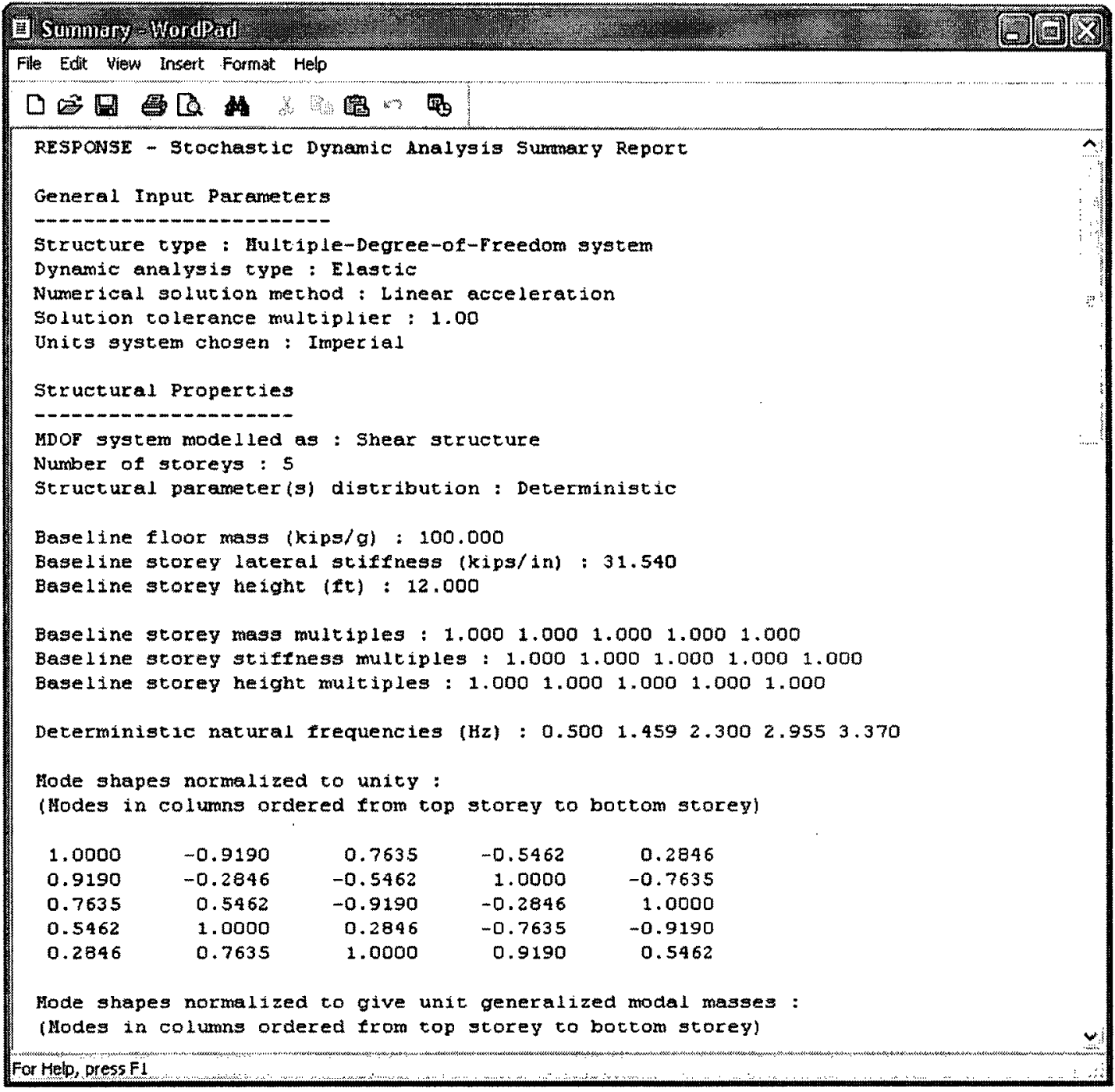

Figure 4.41: Analysis Summary Report 


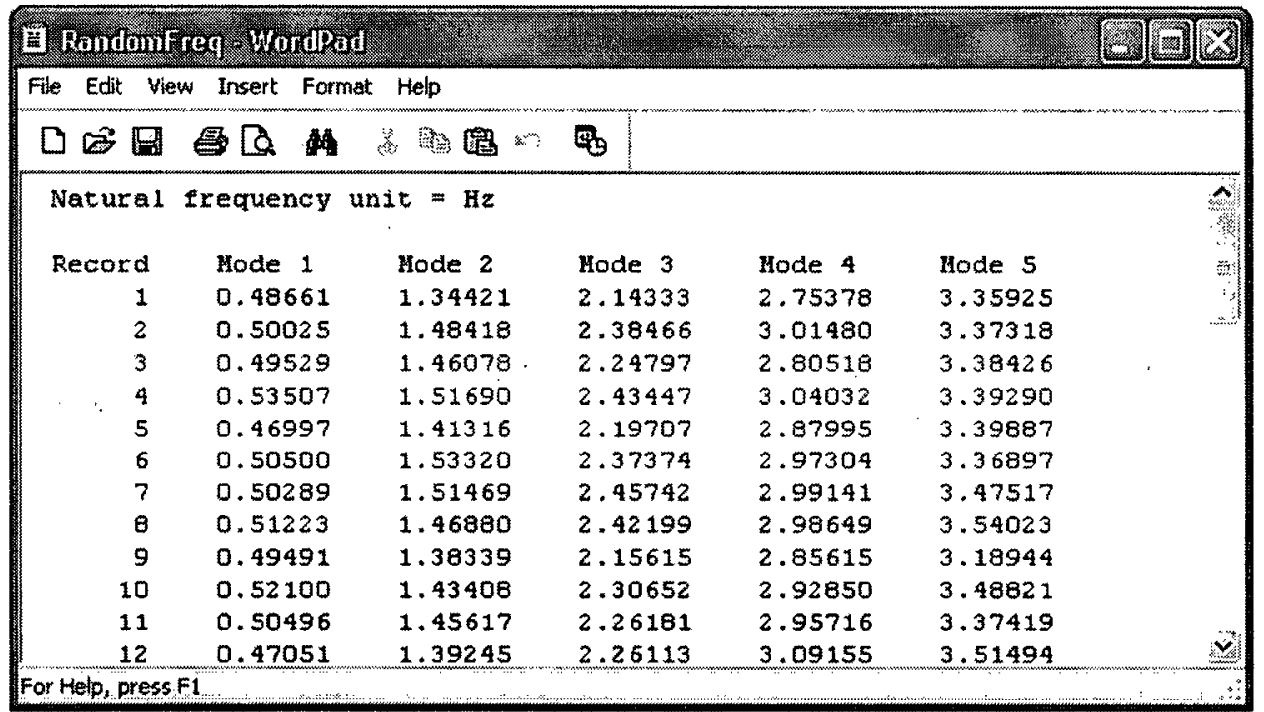

Figure 4.42: Random Natural Frequency Array Printed Output

\begin{tabular}{|c|c|c|c|c|c|}
\hline \multicolumn{2}{|c|}{$\begin{array}{l}\text { gloreys nenlacenient - wardp } \\
\text { Fite Edit View insert format Help }\end{array}$} & & & & \\
\hline \multicolumn{6}{|c|}{ 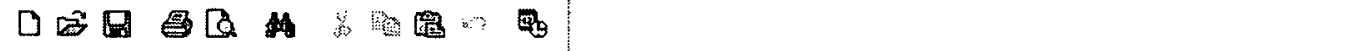 } \\
\hline \multicolumn{3}{|c|}{ Output response units : in } & & & $A$ \\
\hline Time (s) & Kode 1 & Hode 2 & Node 3 & Tocal & \\
\hline $0.000 e+000$ & $0.000000 e+000$ & $0.000000 e+000$ & $0.000000 e+000$ & 0.000000 etoog & \\
\hline $2.000 e-002$ & $-3.020394 \mathrm{e}-004$ & $8.623271 \mathrm{e}-005$ & $-3.711178 e-005$ & $-2.529185 e-004$ & \\
\hline 4.000e-002 & $-1.377908 \mathrm{e}-003$ & $3.898698 \mathrm{e}-004$ & $-1.655512 \mathrm{e}-004$ & $-1.153689 \mathrm{e}-003$ & \\
\hline $6.000 e-002$ & $-3.133410 e-003$ & $8.726570 \mathrm{e}-004$ & $-3.622326 e-004$ & $-2.622986 \mathrm{e}-003$ & \\
\hline $8.000 e-002$ & $-5.327846 e-003$ & $1.449877 \mathrm{e}-003$ & $-5.808864 e-004$ & $-4.458856 \mathrm{e}-003$ & \\
\hline 1.000 e-001 & $-8.293011 \mathrm{e}-003$ & $2.198727 e-003$ & $-8.455147 e-004$ & $-6.939798 \mathrm{e}-003$ & \\
\hline $1.200 e-001$ & $-1.264193 \mathrm{e}-002$ & $3.271097 \mathrm{e}-003$ & $-1.210825 e-003$ & $-1.058166 \mathrm{e}-002$ & \\
\hline 1. $400 e-001$ & $-1.862458 e-002$ & $4.704439 e-003$ & $-1.678402 \mathrm{e}-003$ & $-1.559855 \mathrm{e}-002$ & \\
\hline $1.600 e-001$ & $-2.577589 e-002$ & $6.321739 \mathrm{e}-003$ & $-2.154656 \mathrm{e}-003$ & $-2.160881 e^{-002}$ & \\
\hline 1. $800 e-001$ & $-3.327581 e-002$ & $7.842227 \mathrm{e}-003$ & $-2.504500=-003$ & $-2.793808 e-002$ & \\
\hline $2.000 e-001$ & $-4.074166 e-002$ & $9.117189 \mathrm{e}-003$ & $-2.660099 e-003$ & $-3.428457 e-002$ & \\
\hline $2.200 e-001$ & $-4.866057 \mathrm{e}-002$ & $1.025631 e-002$ & $-2.666943 e-003$ & $-4,107070 e-002$ & \\
\hline $2.400 e-001$ & $-5.794847 e-002$ & $1.149560 \mathrm{e}-002$ & $-2.622278 e-003$ & $-4.907515 e-002$ & \\
\hline $2.600 e-001$ & $-6.896677 \mathrm{e}-002$ & $1.290834 \mathrm{e}-002$ & $-2.586275=-003$ & $-5.864470 e-002$ & \\
\hline 2. $800 \mathrm{e}-001$ & $-8.098102 e-002$ & $1.425050 e-002$ & $-2.506699 \mathrm{e}-003$ & $-6.923721 \mathrm{e}-002$ & \\
\hline $3.000 e-001$ & $-9.317560 e-002$ & $1.526140 \mathrm{e}-002$ & $-2.297541 e-003$ & $-8.021174 e-002$ & \\
\hline $3.200 e-001$ & $-1.054097 e-001$ & $1.588742 \mathrm{e}-002$ & $-1.968026 e-003$ & $-9.149034 e-002$ & \\
\hline $3.400 e-001$ & $-1.174991 \mathrm{e}-001$ & $1.607881 e-002$ & $-1.531394 e-003$ & $-1.029516 e-001$ & \\
\hline $3.600 e-001$ & $-1.293071 e-001$ & $1.585555 \mathrm{e}-002$ & $-1.014835 \mathrm{e}-003$ & $-1.144664 \mathrm{e}-001$ & \\
\hline $3.800 e-001$ & $-1.412986 e-001$ & $1.537967 e-002$ & $-5.248515 e-004$ & $-1.264438 \mathrm{e}-001$ & \\
\hline $4.000 e-001$ & $-1.542344 \mathrm{e}-001$ & $1.985375 e-002$ & $-1.996721 e-004$ & $-1.395804 e-001$ & \\
\hline $4.200 e-001$ & $-1.688670 \mathrm{e}-001$ & $1.452709 e-002$ & $-1.545134 e-004$ & $-1.544945 e-001$ & \\
\hline $4.400 e-001$ & $-1.847443 e-001$ & $1.42972 .5 e-002$ & $-3.109103 \mathrm{e}-004$ & $-1.707580 \mathrm{e}-001$ & \\
\hline 4. $600 e-001$ & $-1.990253 e-001$ & $1.337904 \mathrm{e}-002$ & $-3.446192 e-004$ & $-1.859909=-001$ & \\
\hline $48 \cap n=-n n y$ & $-\because n 936 R \Omega-n n y$ & $11159352-n n 2$ & $-1 \quad 372915-n \cap 4$ & $-1 \quad 8834 n 7=-n n 1$ & 4 \\
\hline
\end{tabular}

Figure 4.43: Example Response Time-History Printed Output (Elastic Analysis) 


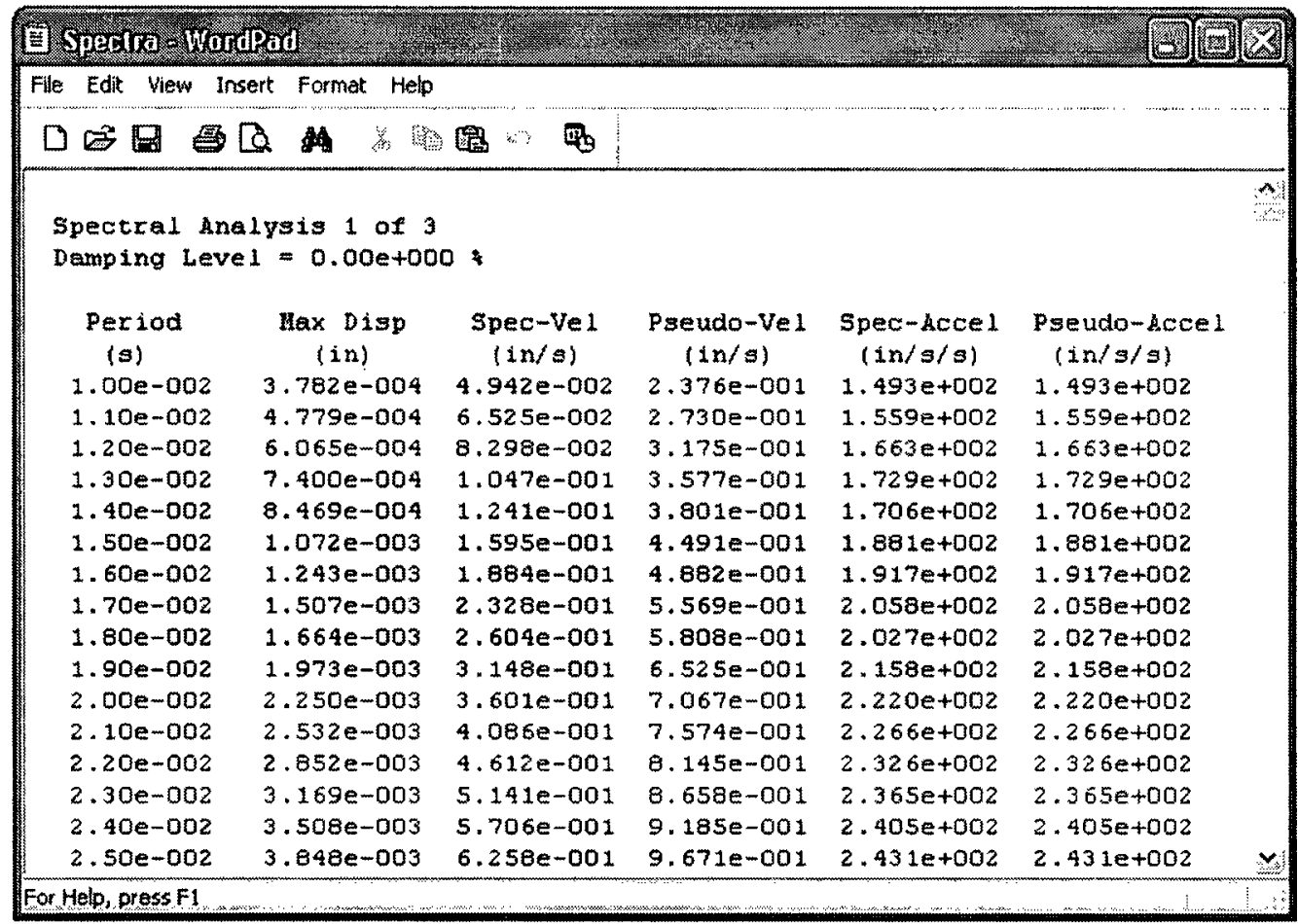

Figure 4.44: Response Spectra Data Printed Output

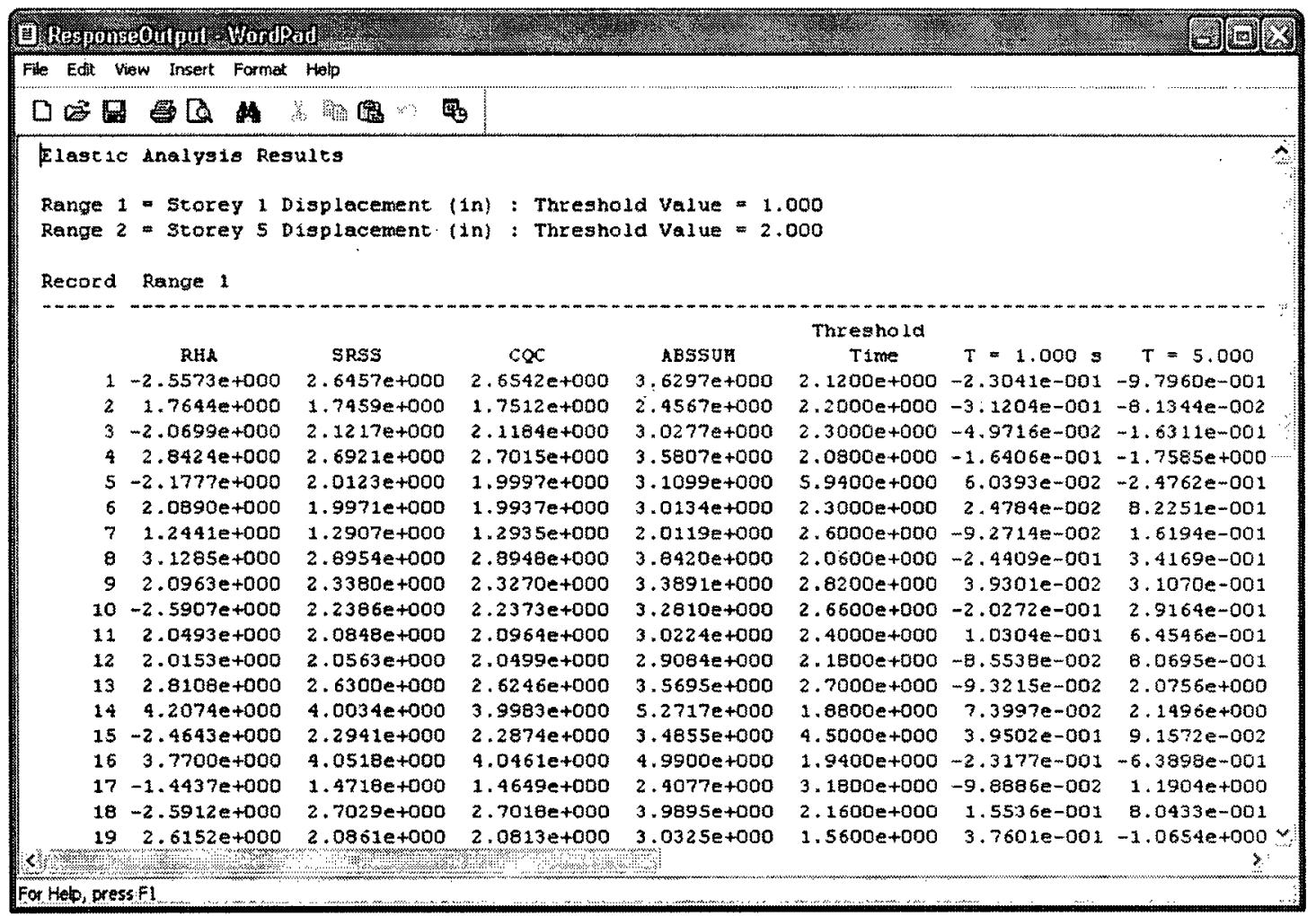

Figure 4.45: Example Multiple Record Analysis Data Printed Output (Elastic Analysis) 


\section{CHAPTER 5}

\section{SOFTWARE VERIFICATION AND CASE STUDIES}

\subsection{INTRODUCTION}

The final stage in any software development process is, of course, verification of the efficacy of the software application. In the case of numerical software, this requires that the calculated output match, to within some subjective tolerance, the corresponding output from an accepted benchmark, which may be a theoretical result, experimental data or results from a similar software model. Once a suitable benchmark has been established and the software has been verified to adequately match that benchmark, it may be released for use as a beta version with the expectation that user testing will identify useful upgrades and problems not identified in initial testing.

The issues related to selecting a suitable benchmark for evaluating structural dynamic analysis results are discussed at the beginning of Section 5.2 followed by several subsections that then use the chosen benchmarks to evaluate various elastic and inelastic PSResponse analysis results. Section 5.3 then summarizes two case studies that were completed as the first application of the beta release of PSResponse. The first case study analyzes the effect of random properties on the dynamic response of a five-storey elastic structure while the second case study analyzes the well-known equal displacement observation in structural dynamics as well as the effect of hysteresis model properties on displacement response.

\subsection{SOFTWARE VERIFICATION}

Verification of numerical software requires that an accepted benchmark be established against which calculated results may be compared. In the case of structural 
dynamic analysis, particularly non-linear time-history analysis, establishing a benchmark must typically rely on experimental data or reasonable agreement between independent software applications, rather than on theoretical results. This is because a complete theoretical structural dynamic response over the time-history of an excitation is not available per se in circumstances involving a non-linear force-deformation relationship and/or response velocity sign reversals. In these situations, the theoretical response assumes perfect conformity to the true force-deformation curve, which requires perfect convergence in the iterative residual force process of each time-step, and perfect identification of response velocity sign reversal points. Clearly, a certain accepted level of numerical error will always be present at every time-step and will tend to accumulate over the duration of a time-history analysis due to the recursive nature of time-stepping methods. Therefore, since the theoretical dynamic response of a structure cannot be separated from the inherent calculation error, software verification must be based on comparison with experimental data or similar software models.

Of the two remaining verification options, the next best choice would typically be verification against experimental data since comparing two software models is subject to compounding of each individual model error. This may result in a situation where the difference between two models is deemed acceptable while the real error in the subject software, as compared to a nominally theoretical result, is the unacceptable sum of the individual model errors. Verification against experimental data avoids the problem of error compounding and is the ultimate test of any mathematical model. However, for this software, comparison of a calculated acceleration response time-history against shaketable data would largely be an exercise in evaluating the efficiency of the built-in system identification algorithm. This is because for a given experimental acceleration response time-history, the software will fit the parameters of the smooth hysteresis model such that the calculated acceleration response time-history matches the input as closely as possible (Sec. 3.3.4.4). While a close match validates the fundamental algorithmic structure and shows that a certain combination of hysteresis parameters was able to match the input acceleration record, it does not necessarily verify that for a different set of structural hysteresis parameters, which may be entered directly to model a desired yield 
displacement, the calculated dynamic response is correct. Of course, one may identify the hysteresis parameters from experimental data collected using a selected base motion history and then compare the calculated and experimental response of a nominally identical structure using the same hysteresis parameters and a different base motion history. This, however, will inevitably result in differences that can be attributed to one or several of the following causes: (i) the nominally identical structure was not identical and, therefore, was modeled with a mismatched set of hysteresis parameters; (ii) the structure was identical but the identified hysteresis parameters are not valid for a different base motion history; (iii) the software model is flawed. The first possible error source is likely not significant and may be quantified by comparing the experimental response of several nominally identical structures using the same base motion history as was used for hysteresis parameter identification. This allows the effect of small structural differences on dynamic response to be evaluated independently of a software flaw or the effect of a different base motion history. The second and third possible error sources are much more likely to be responsible for any observed differences between an experimental response and the calculated response, unfortunately, it is impossible to separate the two. Even if a new set of hysteresis parameters is identified from the experimental response using the different base motion history, it is unclear whether the difference in the hysteresis parameter sets is strictly due to an inherent need to adjust the parameters for each different base motion history or there is a flaw in the software. Therefore, the only way to verify the accuracy of PSResponse is to directly enter a set of basic hysteresis parameters corresponding to a specific yield displacement and compare results with a similar software application and other published results. While this approach is subject to the aforementioned error compounding problem, as well as difficulties in setting up identical dynamic analyses using different software models, the intent is simply to perform a so-called "sanity check" to verify that PSResponse provides dynamic analysis results that are reasonably similar to an accepted industry standard software package.

Following the decision to use the results of a similar software package as the verification benchmark, an accepted industry standard software package needed to be chosen from a list of possible candidates including; SAP, ETABS, DRAIN, CANNY, and 
RAUMOKO among others. Based on consideration of the industry acceptance of each candidate as well as preliminary attempts to use each software package, $\boldsymbol{S A P 2 0 0 0}$ Nonlinear 8.1.2 (Computers \& Structures Inc. 2003) was chosen as the software benchmark. It is the most recent version of a widely-used engineering analysis and design package, first developed in 1975, which is capable of performing highly sophisticated non-linear time-history analysis.

Finally, as a further verification check, results from each software application were checked against results published by Chopra (1995). All results, which were obtained from both elastic and inelastic dynamic analysis of single-degree-of-freedom (SDOF) and multiple-degree-of-freedom (MDOF) structures, are summarized in the following sections.

\subsubsection{Elastic Analysis}

Verification of elastic dynamic analysis results is a relatively straightforward process, as compared to inelastic dynamic analysis results, since elastic analysis does not involve an iterative residual force process and does not depend on the assumed hysteretic behaviour of the structure. Without those sources of discrepancy, elastic analysis results should be almost identical amongst all software applications.

\subsubsection{Single-Degree-of-Freedom Elastic Systems}

Beginning with SDOF systems, Figure 5.1 and Table 5.1 show the PSResponse and $S A P 2000$ calculated deformation response of several natural frequency and damping combinations to the north-south component of the 1940 Imperial Valley, California earthquake recorded at $\mathrm{El} \mathrm{Centro,} \mathrm{hereafter} \mathrm{referred} \mathrm{to} \mathrm{as} \mathrm{the} \mathrm{El}$ Centro ground motion. Included in Table 5.1 are Chopra's peak deformation values taken from results given as Figure 6.4.1. There is very good agreement between all results, with $S A P 2000$ and Chopra being virtually identical while PSResponse gave slightly different peak deformations. The difference is likely due to the way in which the overshoot problem 
(Sec. 3.3.2) is dealt with in each set of results. It appears that the SAP2000 and Chopra results do not reflect a subdivision of time-steps to determine the point of zero velocity when a velocity sign reversal occurs.

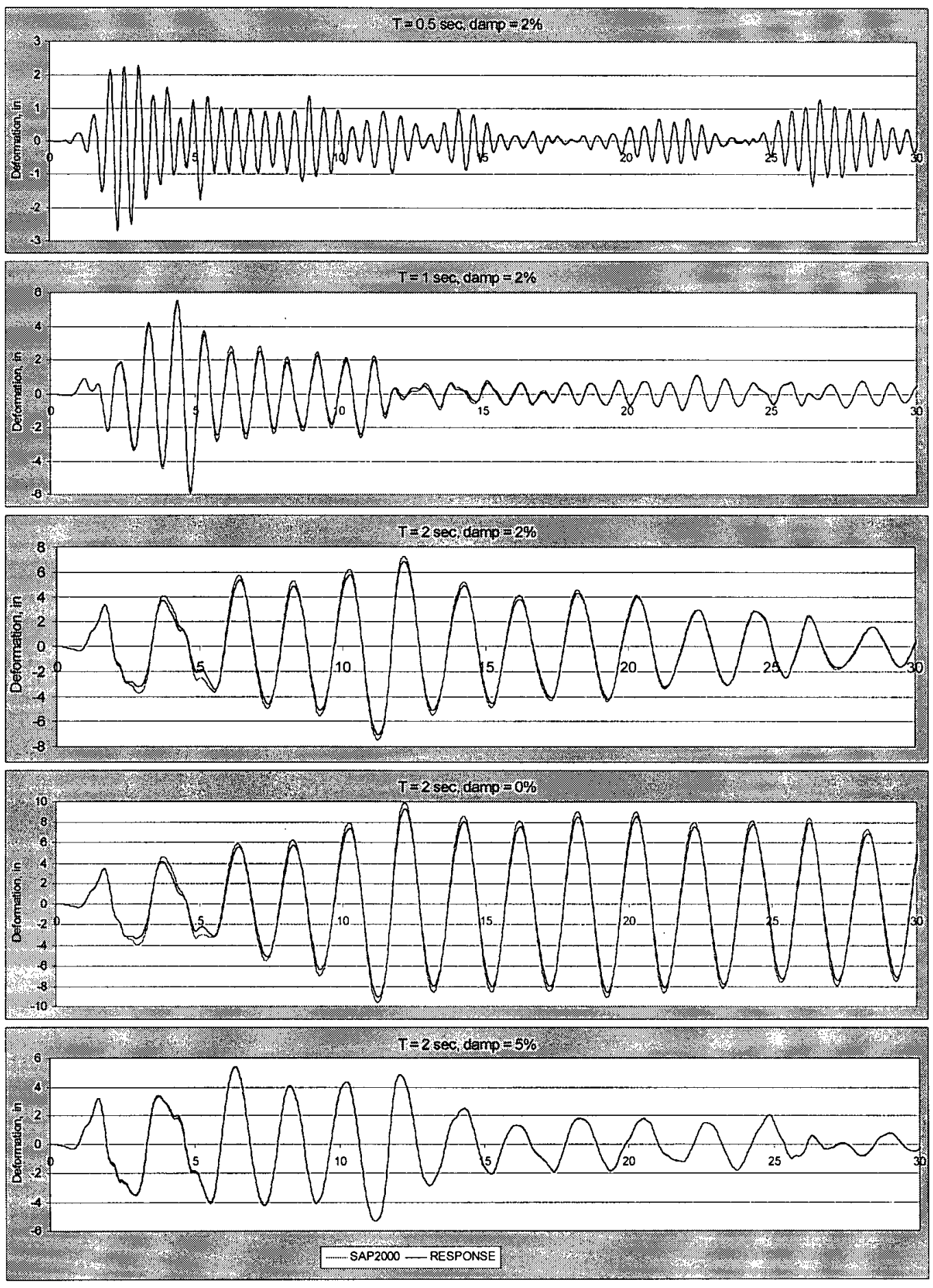

Figure 5.1: Elastic Deformation Response of SDOF Systems - PSResponse vs. SAP2000 
When this feature of PSResponse was disabled, all three sets of results were virtually identical.

Table 5.1: Comparison of Peak Deformation Responses of SDOF Systems (in.)

\begin{tabular}{|l|c|c|c|}
\hline System & PSResponse & SAP2000 & Chopra \\
\hline $\mathrm{T}=0.5 \mathrm{sec}, \zeta=2 \%$ & -2.70 & -2.67 & -2.67 \\
\hline $\mathrm{T}=1 \mathrm{sec}, \zeta=2 \%$ & -5.72 & -5.97 & -5.97 \\
\hline $\mathrm{T}=2 \mathrm{sec}, \zeta=2 \%$ & -7.06 & -7.47 & -7.47 \\
\hline $\mathrm{T}=2 \mathrm{sec}, \zeta=0 \%$ & 9.29 & 9.92 & 9.91 \\
\hline $\mathrm{T}=2 \mathrm{sec}, \zeta=5 \%$ & 5.43 & 5.37 & 5.37 \\
\hline
\end{tabular}

\subsubsection{Multiple-Degree-of-Freedom Elastic System}

To verify elastic analysis results for MDOF systems, the five-storey shear frame shown in Figure 5.2 was analyzed to allow comparison with detailed results given by Chopra as Example 13.2.6 and Example 13.8.2. Figure 5.3 and Table 5.2 illustrate the close agreement between each set of software results and Chopra's published results for several selected response quantities related to the El Centro ground motion.

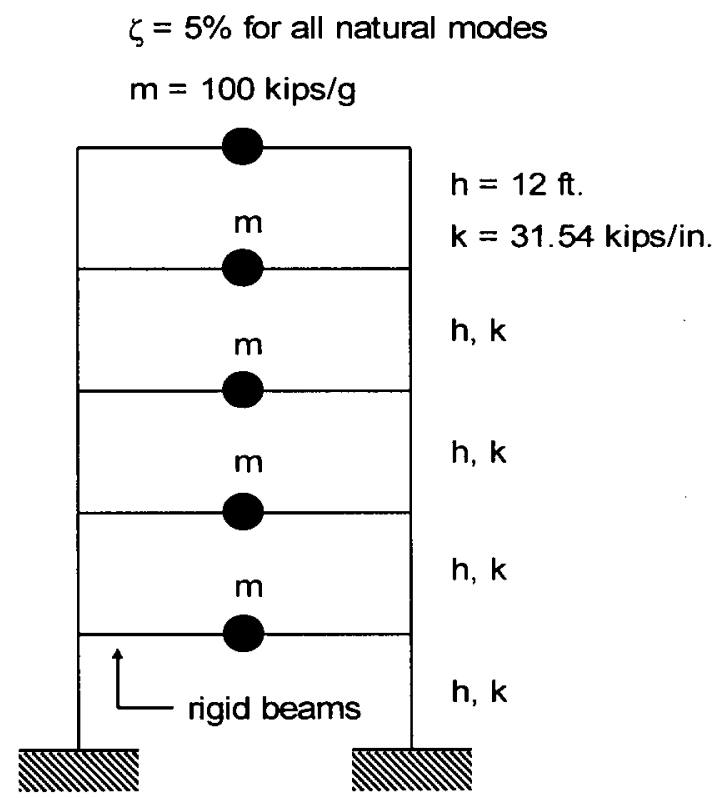

Figure 5.2: Five-Storey Elastic Shear Frame 

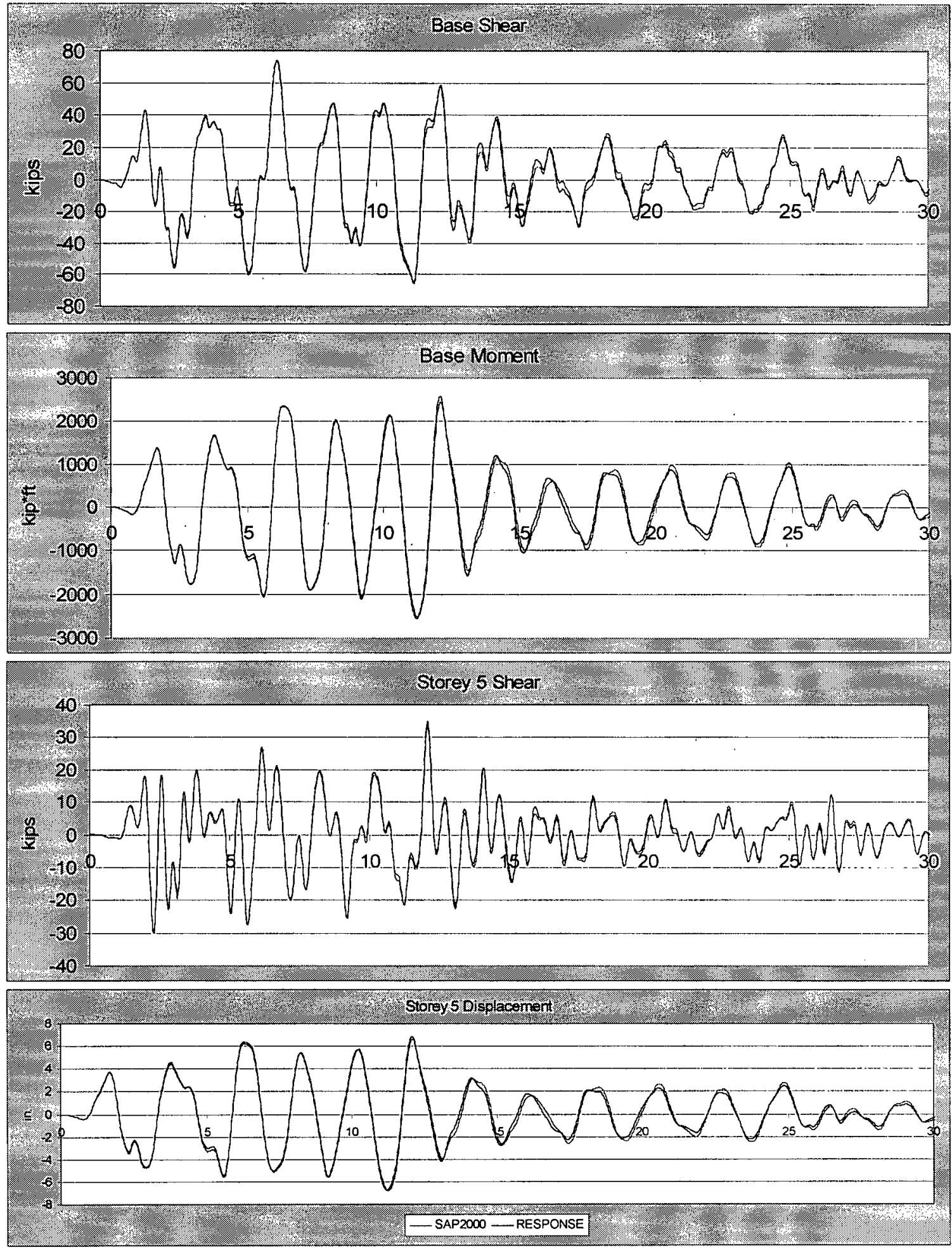

Figure 5.3: Elastic Responses of Five-Storey Shear Frame - PSResponse vs. SAP2000 
Table 5.2: Comparison of Elastic Peak Responses of Five-Storey Shear Frame

\begin{tabular}{|c|c|c|c|c|c|}
\hline $\begin{array}{l}\text { ModeMrodal } \\
\text { Combination }\end{array}$ & $\begin{array}{l}\text { Result } \\
\text { Source }\end{array}$ & $\begin{array}{c}\text { Base } \\
\text { Shear } \\
\text { Shas) }\end{array}$ & $\begin{array}{l}\text { Base Moment } \\
(\text { (kip'st })\end{array}$ & $\begin{array}{l}\text { Storey } 5 \\
\text { Shear (kips) }\end{array}$ & $\begin{array}{l}\text { Storey } 5 \\
\text { Displacement (in.) }\end{array}$ \\
\hline \multirow{2}{*}{ Mode 1} & PSResponse & 61.021 & 2572.637 & 17.368 & 6.797 \\
\hline & Chopra & 60.469 & 2549.400 & 17.211 & 6.731 \\
\hline \multirow{2}{*}{ 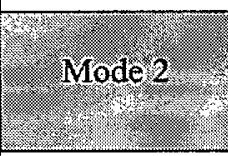 } & PSRRpionse & 24.174 & -349 & -20.0 & -0.922 \\
\hline & Chopra & 20.533 & -354.330 & -20.382 & 0.936 \\
\hline \multirow{2}{*}{ Mode 3} & PSResponse & -9.864 & -90.376 & -12.919 & -0.239 \\
\hline & Chopra & -9.867 & -90.402 & -12.923 & -0.239 \\
\hline \multirow{2}{*}{ Mode 4} & PSResponse & -2908 & $\overline{2}$ & 4.8 & 0.0 \\
\hline & Chopra & x. -2.943 & 20.986 & -4.951 & 40.0 .055 \\
\hline \multirow{2}{*}{ Mode 5} & PSResponse & -0.553 & -3.460 & -1.062 & -0.009 \\
\hline & Chopra & -0.595 & -3.718 & -1.141 & -0.010 \\
\hline \multirow{2}{*}{ ABSSUN } & PSResponse & 98.601 & 303799 & 56.404 & 8.026 \\
\hline & Chopra & 98.407 & 3018.8 & 56.608 & 8.971 \\
\hline \multirow{2}{*}{ SRSS } & PSResponse & 66.470 & 2598.7 & 29.985 & 6.866 \\
\hline & Chopra & 66.066 & 2575.6 & 30.074 & 6.800 \\
\hline \multirow{2}{*}{$\frac{1}{90 \mathrm{cos}}$} & PSResponse & 66.396 & 25968 & 30,701 & 6.861 \\
\hline & Chopra & 66.507 & $(25722$ & 29.338 & 6.793 \\
\hline \multirow{3}{*}{ RHA } & PSResponse & 74.030 & 2587.5 & 34.970 & 6.836 \\
\hline & Chopra & 73.278 & 2593.2 & 35.217 & 6.847 \\
\hline & SAP2000 & 74.376 & -2517.6 & 33.716 & -6.806 \\
\hline
\end{tabular}

The preceding elastic dynamic analysis results given in Table 5.1, Figure 5.1, Table 5.2 and Figure 5.3 indicate that there is very good agreement between each independent set of results for both single-degree-of-freedom elastic systems and a multiple-degree-of-freedom elastic system. This is taken as evidence that PSResponse results meet an acceptable standard of accuracy for elastic dynamic analysis of structures. 


\subsubsection{Inelastic Analysis}

Unlike elastic dynamic analysis, inelastic dynamic analysis depends on the assumed hysteretic behaviour of the structure, assumed post-yield behaviour and convergence of an iterative residual force process at each time-step. To allow a comparison of results with SAP2000 and results published by Chopra (1995), PSResponse hysteretic behaviour for each time-history analysis was constrained to remain undamaged with no pinching and no progressive strength or stiffness degradation. Yield displacement or drift, which must be specified in PSResponse when the default non-degrading, non-pinching behaviour is selected, was determined for each analysis from the non-linear dynamic analysis examples given by Chopra, which serve as PSResponse verification cases. In SAP2000, yielding behaviour is based on a userspecified yield stress for each structural element such as a beam or column. These yield stresses were adjusted in each analysis to limit all shear forces to the elastoplastic limits imposed in the Chopra examples.

\subsubsection{Single-Degree-of-Freedom Inelastic Systems}

Beginning again with SDOF systems, Figure 5.4, Figure 5.5, Figure 5.6 and Table 5.3 show the PSResponse and SAP2000 calculated responses of systems with identical elastic properties but different normalized yield strengths to the El Centro ground motion, where the normalized yield strength is equivalent to the ratio of yield displacement to peak elastic displacement. Included in Table 5.3 are Chopra's peak values taken from results given in Section 7.4.1. Note that $\boldsymbol{S A P 2 0 0 0}$ results are not given for the case in which normalized yield strength $=0.125$ because the software indicated that the system had collapsed under the El Centro ground motion. To illustrate the relative hysteretic behaviour of each system, Figure 5.7 shows the calculated energy dissipation for each normalized yield strength, which is the cumulative area of the associated hysteresis loop. Results are presented for PSResponse only since the corresponding results from SAP2000 were not available. 

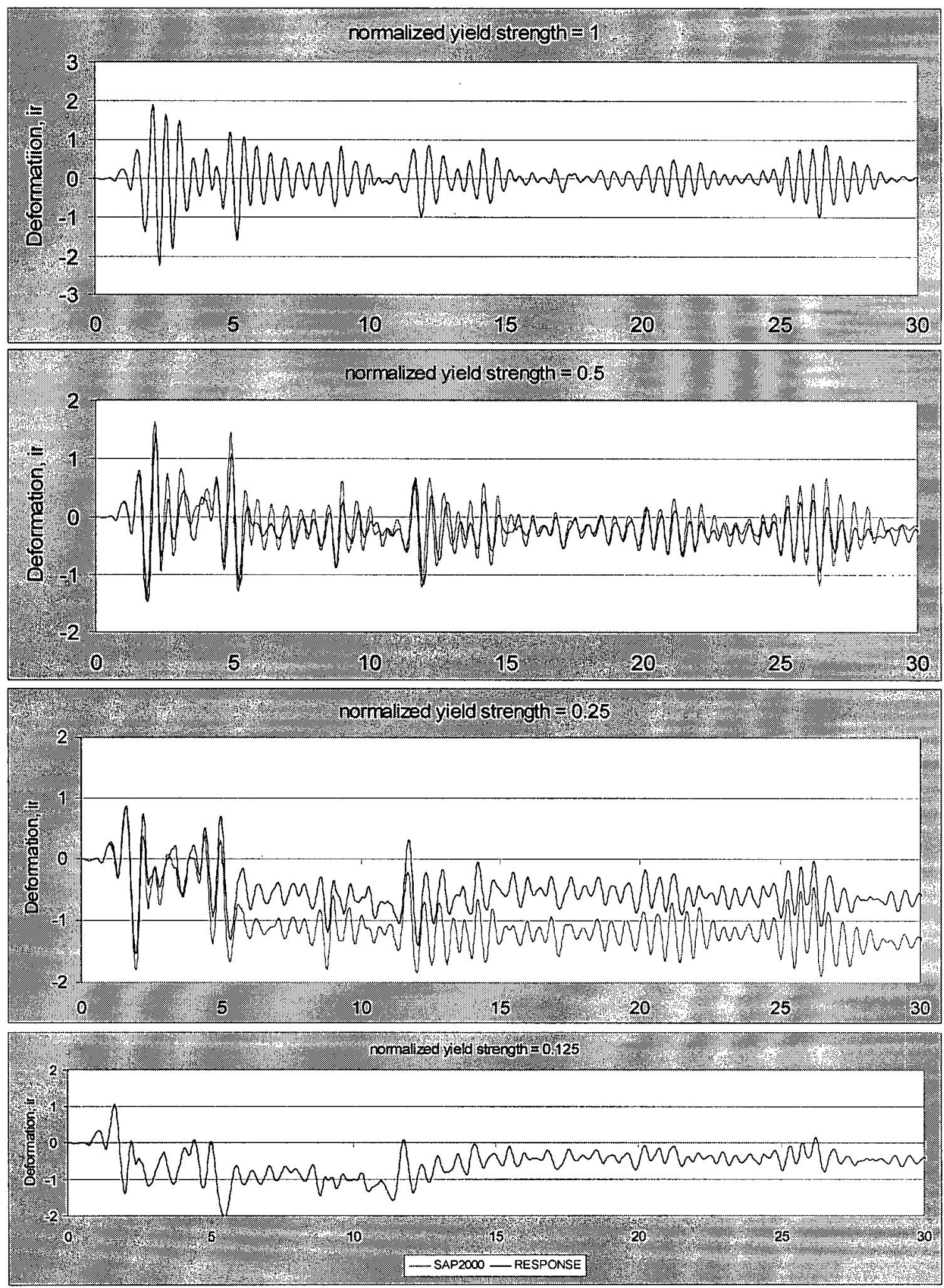

Figure 5.4: Inelastic Deformation Response of SDOF Systems - PSResponse vs. SAP2000 

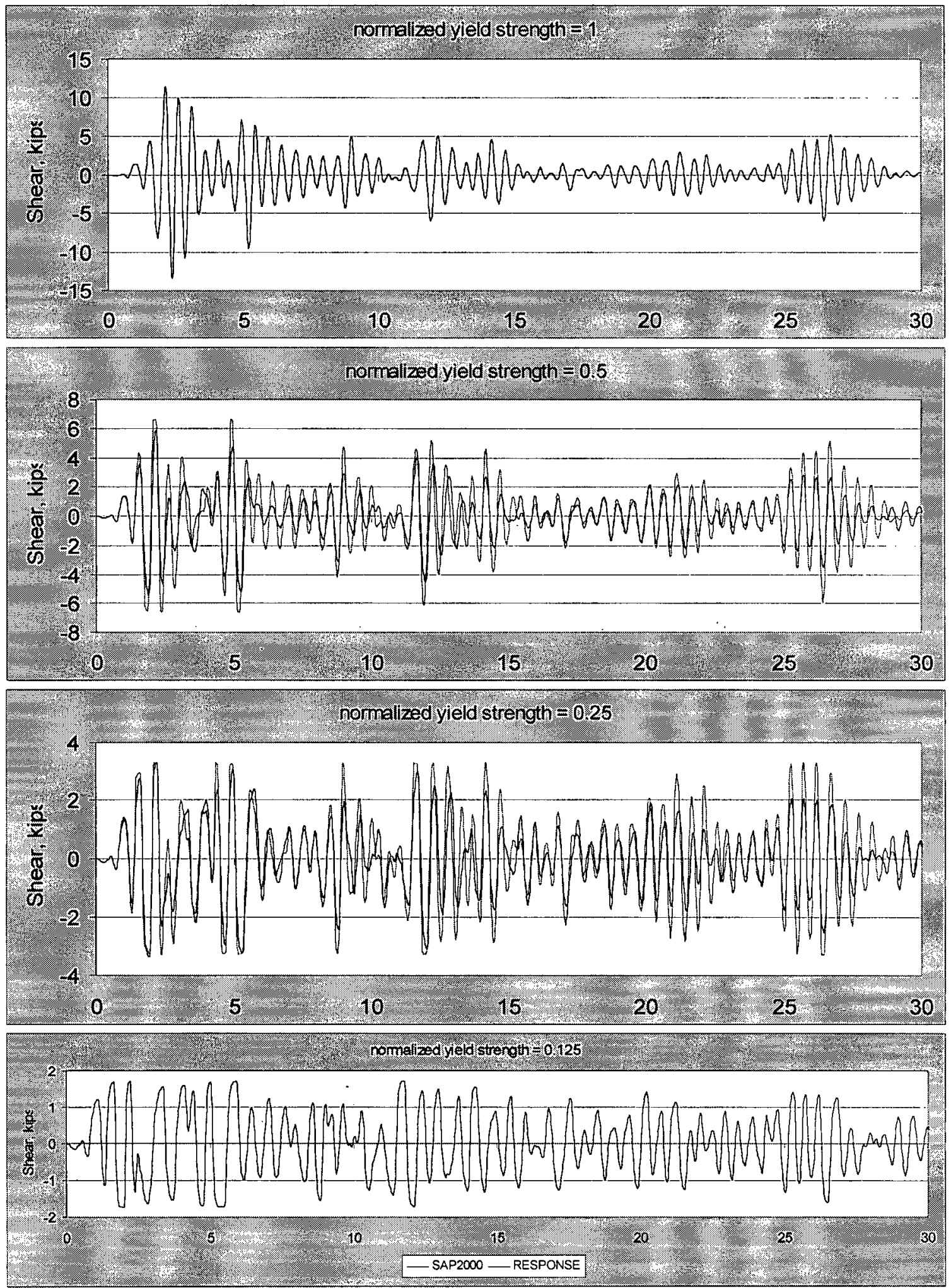

Figure 5.5: Inelastic Shear Response of SDOF Systems - PSResponse vs. SAP2000 

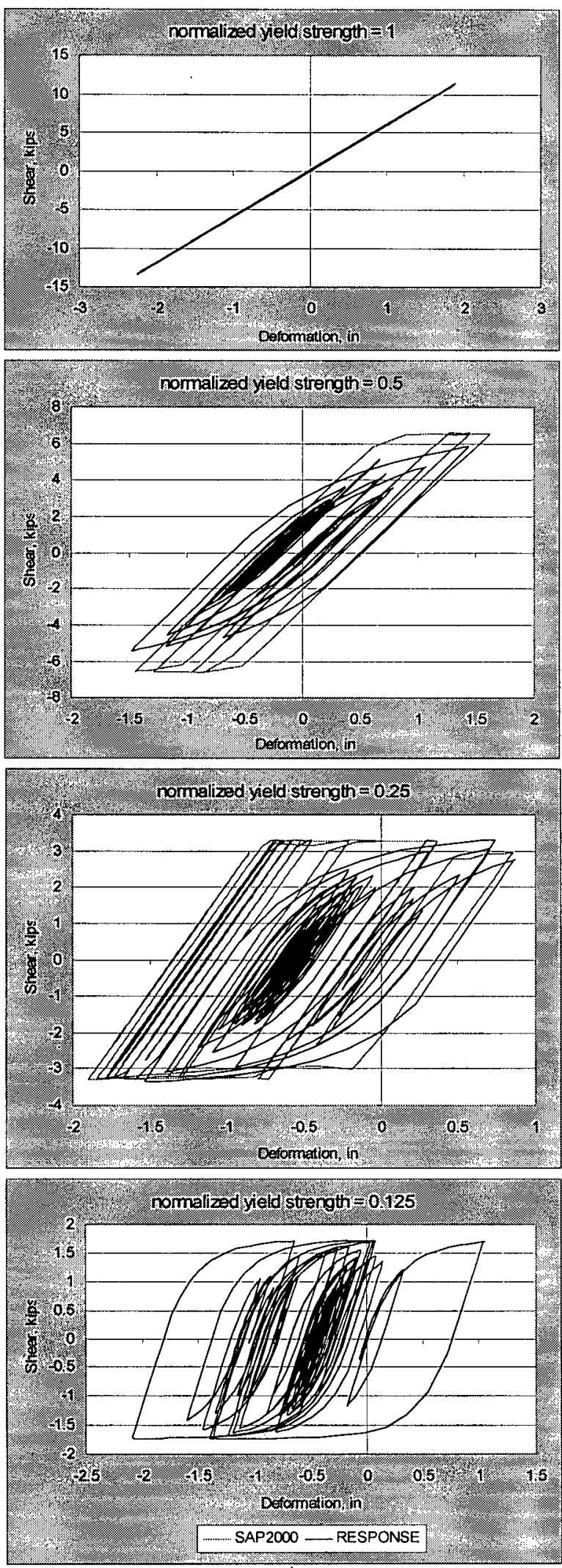

Figure 5.6: Hysteresis Loops of SDOF Systems - PSResponse vs. SAP2000 
Table 5.3: Comparison of Inelastic Peak Responses of SDOF Systems ( $\mathrm{T}=0.5 \mathrm{sec}$, Damp $=5 \%$ )

\begin{tabular}{|c|c|c|c|c|c|c|c|c|}
\hline & \multicolumn{2}{|c|}{ Norm Yield $=1$} & \multicolumn{2}{|c|}{ Norm Yield $=0.5$} & \multicolumn{2}{|c|}{ Norm Yield $=0.25$} & \multicolumn{2}{|c|}{ Norm Yield $=0.125$} \\
\hline & $\begin{array}{l}\text { Displ } \\
(\mathrm{in})\end{array}$ & $\begin{array}{l}\text { Shear } \\
\text { (kips) }\end{array}$ & $\begin{array}{l}\text { Displ } \\
\text { (in) }\end{array}$ & $\begin{array}{l}\text { Shear } \\
\text { (kips) }\end{array}$ & $\begin{array}{c}\text { Displ } \\
\text { (in) }\end{array}$ & $\begin{array}{l}\text { Shear } \\
\text { (kips) }\end{array}$ & $\begin{array}{l}\text { Displ } \\
\text { (in) })\end{array}$ & $\begin{array}{l}\text { Shear } \\
\text { (kips) }\end{array}$ \\
\hline PSResponse & -2.25 & -13.45 & -1.48 & 5.84 & -1.54 & -3.37 & -2.09 & -1.74 \\
\hline Chopra & -2.25 & -13.45 & 1.62 & 6.73 & 1.75 & 3.36 & 2.07 & 1.68 \\
\hline SAP2000 & -2.24 & -13.38 & 1.63 & 6.64 & -1.90 & 3.32 & - & - \\
\hline
\end{tabular}

As expected, the preceding inelastic dynamic analysis results in Table 5.3, Figure 5.4, Figure 5.5 and Figure 5.6 show greater differences between software applications than the elastic analysis results presented previously (Sec. 5.2.1.1) due to the increased number of assumptions and sources of numerical error inherent in inelastic dynamic analysis. The primary difference between PSResponse and SAP2000, other than the overshoot issue identified previously, is in the "sharpness" of the yield point, which is clearly illustrated in Figure 5.6. The $\boldsymbol{S A P 2 0 0 0}$ shear forces exhibit classic elastoplastic behaviour, which was also used by Chopra, while the PSResponse shear forces follow smooth curves and do not have well-defined yield points. This is because the default hysteresis parameters in PSResponse were selected to reflect the yield behaviour of most structures, which tends to follow a smooth, continuous curve without abrupt changes in stiffness. For structures that do have a well-defined yield point, such as certain SDOF steel structures, the sharpness of the yield point could be increased in PSResponse with a minor change to the default hysteresis parameters.

In spite of the difference in yield behaviour between models, there is still reasonably good agreement between PSResponse, SAP2000 and the published results of Chopra. Since the intent was simply to perform a "sanity check" to verify that PSResponse provides inelastic dynamic analysis results that are similar to a standard software package, the reasonably good agreement between calculated responses is again taken as evidence that PSResponse results meet an acceptable standard of accuracy for single-degree-of-freedom inelastic structures. 

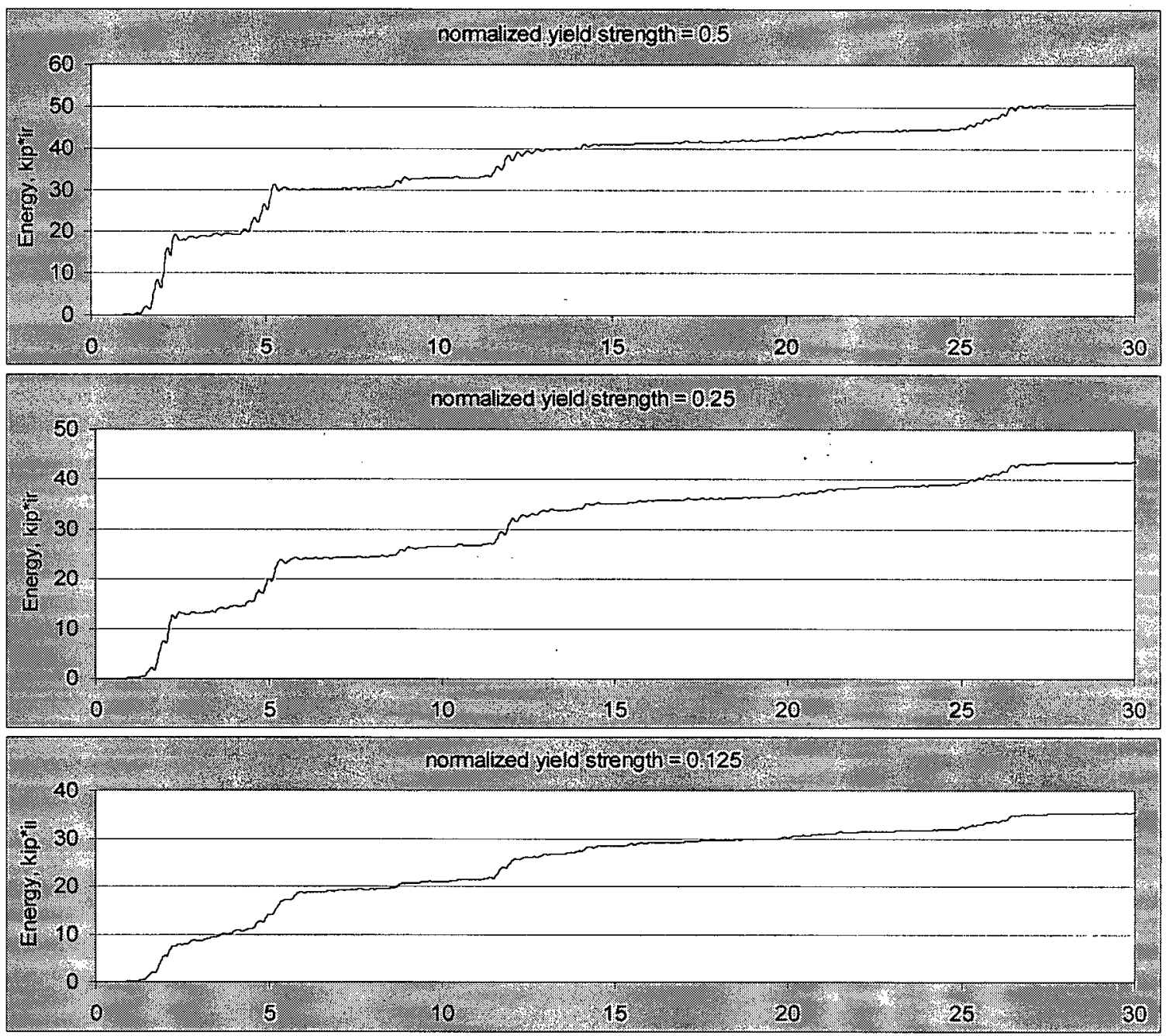

Figure 5.7: Dissipated Energy of SDOF Systems - PSResponse

\subsubsection{Multiple-Degree-of-Freedom Inelastic System}

The final stage in the PSResponse software verification process was the simulation of a multiple-degree-of-freedom structure undergoing inelastic deformation. To verify inelastic dynamic analysis results for MDOF systems, a five-storey shear frame, shown in Figure 5.8, was again analyzed to allow comparison with results given by Chopra in Section 19.1.2 and Section 19.1.3. Table 5.4, Figure 5.9, Figure 5.10 and Figure 5.11 show the PSResponse and SAP2000 calculated storey deformation and shear responses of the frame structure to the El Centro ground motion. To again illustrate relative hysteretic behaviour, Figure 5.12 shows the energy dissipation at each storey 
level for PSResponse only, since the corresponding results from $\boldsymbol{S A P 2 0 0 0}$ were again not available.

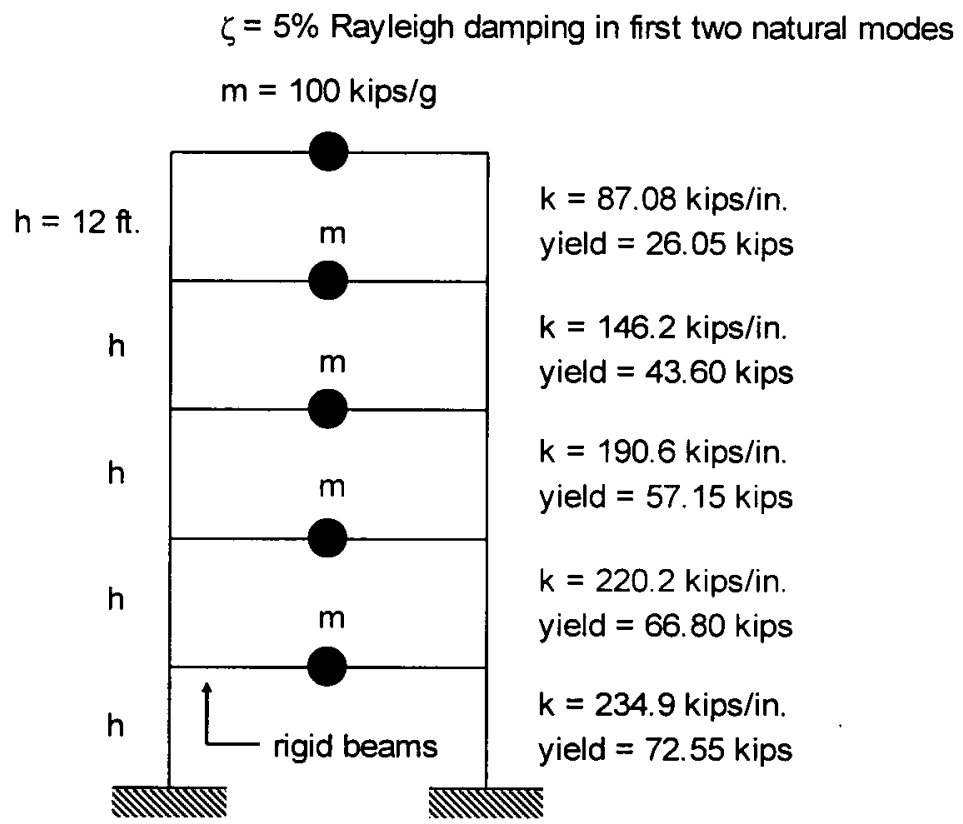

Figure 5.8: Five-Storey Inelastic Shear Frame

Table 5.4: Comparison of Inelastic Peak Responses of Five-Storey Shear Frame

\begin{tabular}{|c|c|c|c|c|}
\hline Storey & Quantity & PSResponse & SAP2000 & Chopra \\
\hline \multirow{3}{*}{1} & Displacement (in.) & -0.78 & -1.05 & -1.20 \\
\hline & Drift (in.) & -0.78 & -1.05 & -1.20 \\
\hline & Shear (kips) & 69.56 & 72.02 & 72.55 \\
\hline \multirow{3}{*}{$\frac{2}{2}$} & Displacement (in) & 1.38 & -2.28 & 2.05 \\
\hline & Drif (in) & -0.71 & -1.23 & $3 .-3$ \\
\hline & Shear (kips) & 60.60 .92 & 2.65 .79 & $=66.80$ \\
\hline \multirow{3}{*}{3} & Displacement (in.) & -2.12 & -2.60 & -2.40 \\
\hline & Drift (in.) & -0.80 & 1.05 & - \\
\hline & Shear (kips) & 52.60 & 57.69 & 57.15 \\
\hline \multirow{3}{*}{$\sqrt[3]{3}$} & Displacement (in) & -2.63 & -2.50 & 2.40 \\
\hline & Drift (in.) & 0.59 & $1911=$ & $x_{0}=0$ \\
\hline & Shear (kips) $\overbrace{}^{2}$ & 40.28 & 44.46 & $y=43.60$ \\
\hline \multirow{3}{*}{5} & Displacement (in.) & -3.08 & -3.48 & -3.20 \\
\hline & Drift (in.) & 0.68 & -1.02 & -0.81 \\
\hline & Shear (kips) & 25.30 & 25.67 & 26.05 \\
\hline
\end{tabular}



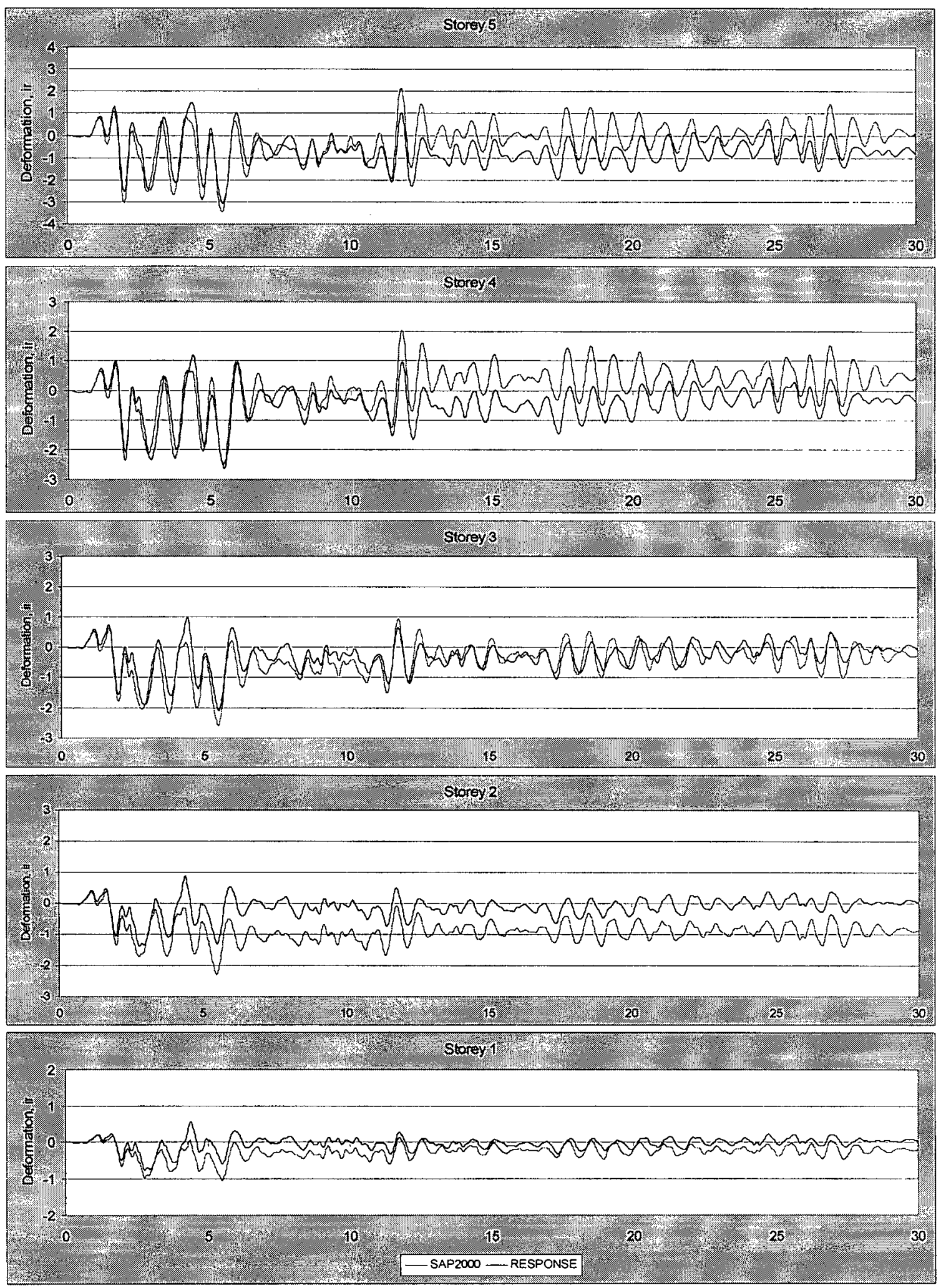

Figure 5.9: Inelastic Deformation Response of Five-Storey Shear Frame - PSResponse vs. SAP2000 

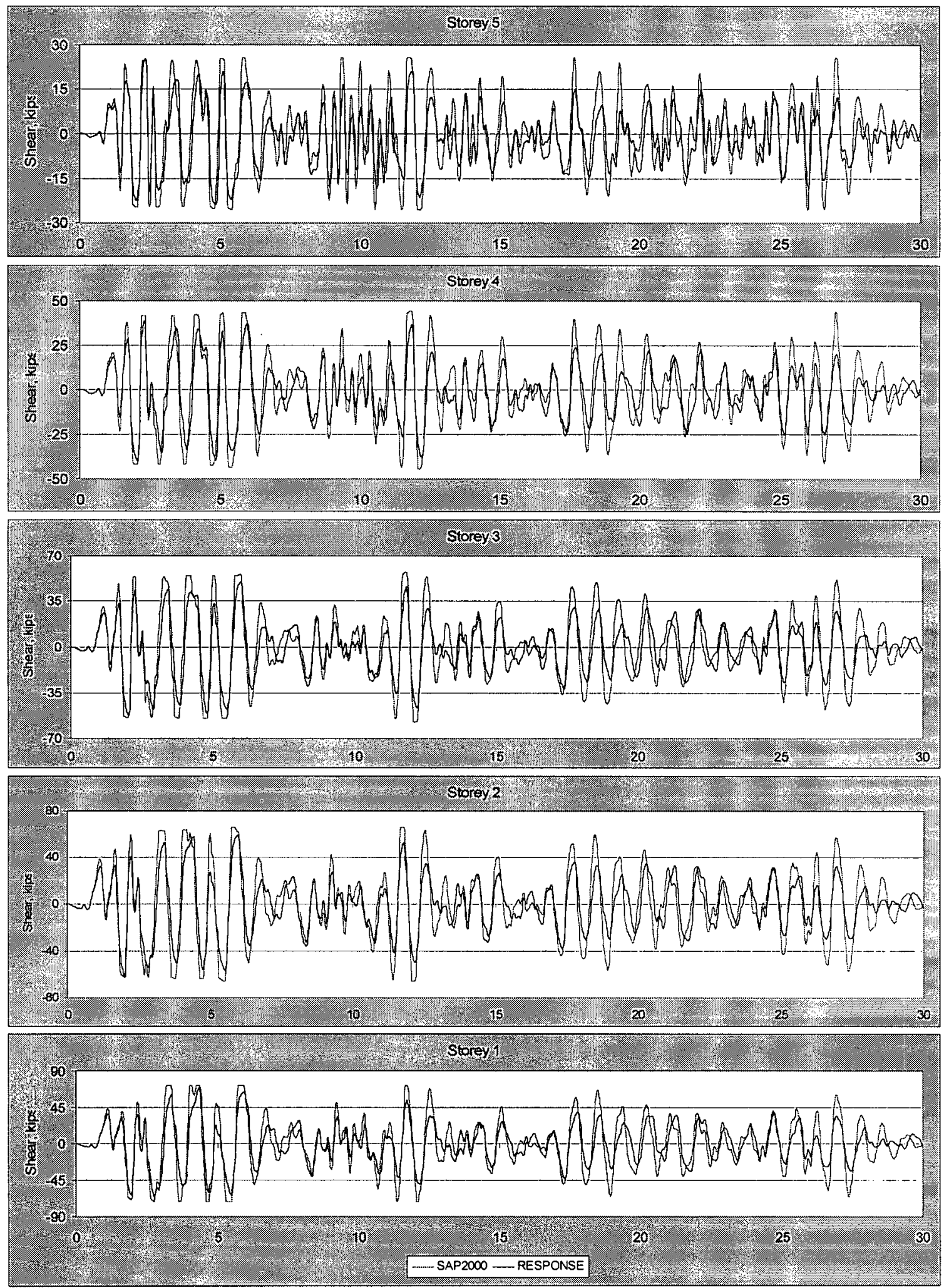

Figure 5.10: Inelastic Shear Response of Five-Storey Shear Frame - PSResponse vs. SAP2000 

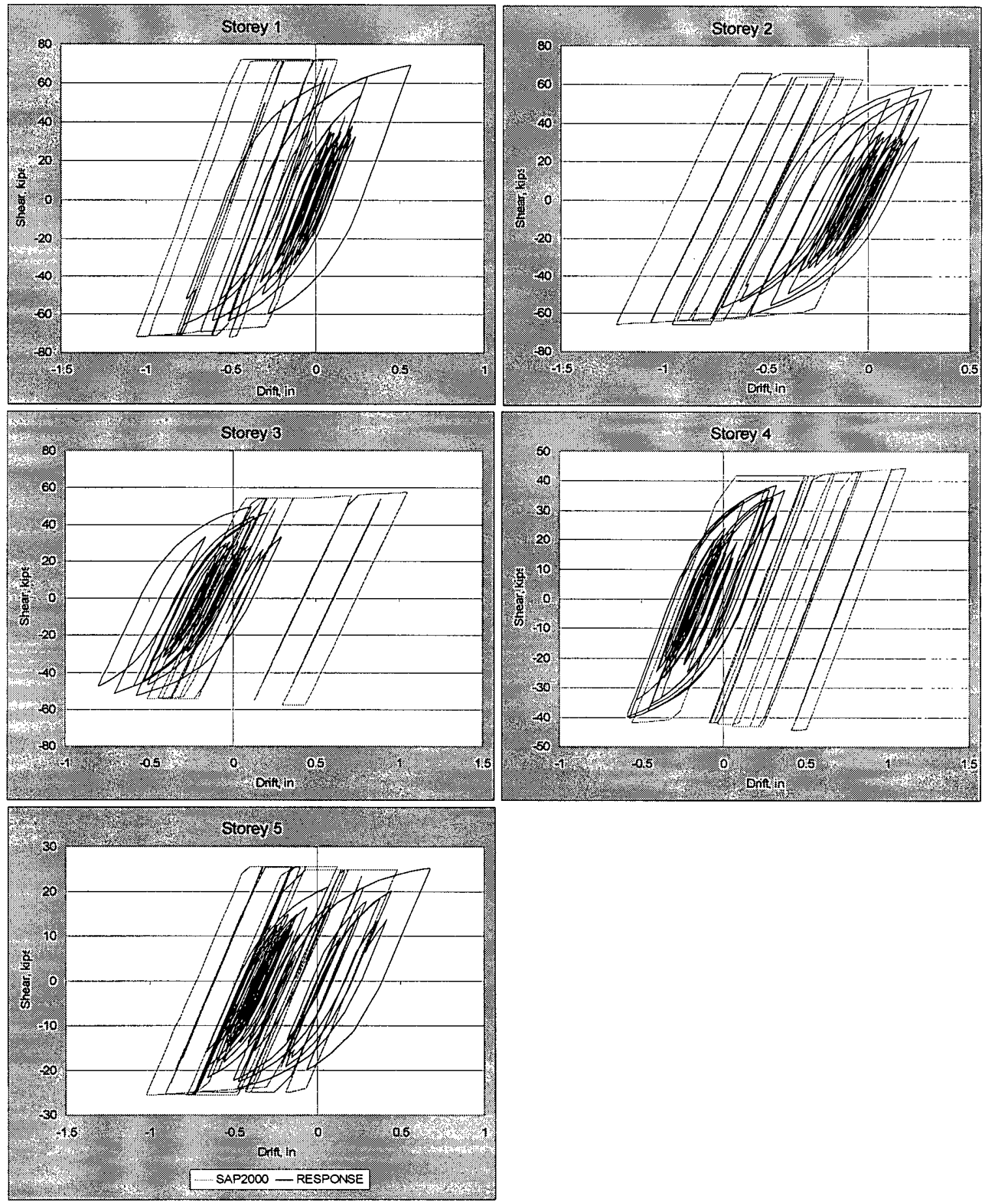

Figure 5.11: Hysteresis Loops of Five-Storey Shear Frame - PSResponse vs. SAP2000 

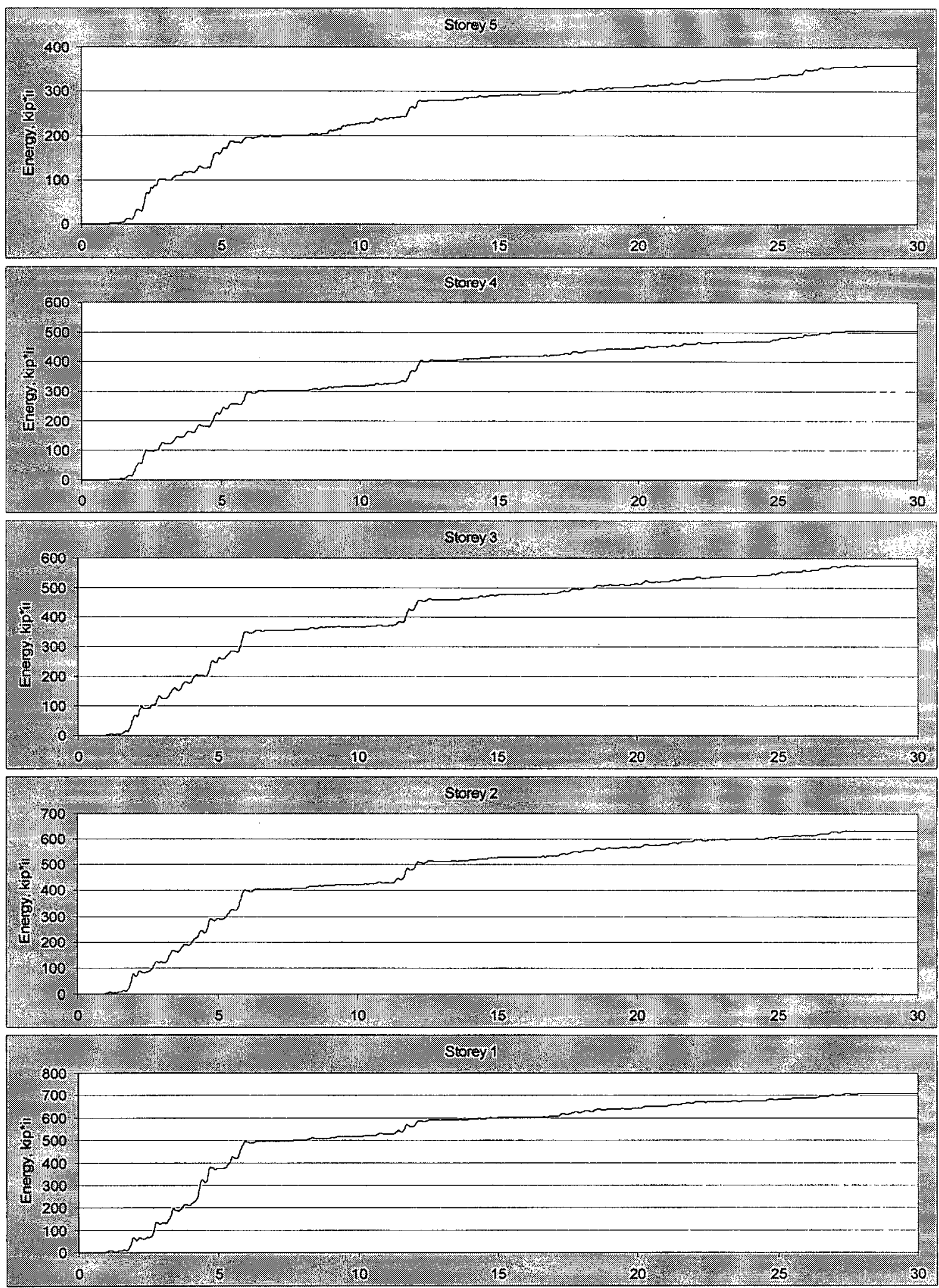

Figure 5.12: Dissipated Energy of Five-Storey Shear Frame - PSResponse 
Similar to SDOF systems, the preceding inelastic dynamic analysis results for a five-storey shear structure subjected to the El Centro ground motion show greater differences between software applications than the elastic analysis results of a similar structure (Sec. 5.2.1.2). This is again due to the additional sources of numerical error and increased number of assumptions inherent in inelastic dynamic analysis as compared to elastic analysis. Differing assumptions regarding yield behaviour and the overshoot problem, which were identified previously (Sec. 5.2.2.1), as well as systemic differences in setting up a dynamic analysis between SAP2000 and PSResponse mean that the analyses will never be exactly comparable. Nevertheless, each independent set of dynamic analysis results for a multiple-degree-of-freedom inelastic structure, and the published results of Chopra, are in reasonably close agreement. This standard of accuracy is considered acceptable for the intended purpose of PSResponse, which is to provide fast, summary-level dynamic analysis results for determining the probabilistic response of linear and non-linear systems under stochastic dynamic loading. To that end, it is worth noting that PSResponse was two orders of magnitude faster than SAP2000 in calculating the inelastic dynamic response of the five-storey shear structure subjected to the El Centro ground motion. Calculation time for PSResponse was less than one second on a $1.5 \mathrm{GHz}$ Pentium 4 computer with $256 \mathrm{MB}$ RDRAM while $\boldsymbol{S A P 2 0 0 0}$, with its much more detailed finite-element basis, took approximately 83 seconds.

\subsection{CASE STUDIES}

The first application of the beta release of PSResponse was the completion of two case studies that were selected from the myriad possibilities in structural dynamics largely to demonstrate the capabilities of the program as a research and analysis tool. These case studies analyzed, from a probabilistic point of view, three general questions pertinent to structural dynamics and earthquake engineering; the relative effect of random structural properties on the dynamic response of a structure, the appropriateness of the well-known equal displacement observation in structural dynamics and the effect of hysteresis model properties on displacement response. Results from the case studies provide answers to those questions for the chosen structural models, input variable ranges 
and output response quantities without attempting to be completely general in nature. Also, comparison of certain results with some of the seismic structural response and reliability studies cited in Section 2.5 was left as future work.

\subsubsection{Case Study \#1}

To assess the relative effect of structural property variability on the random response of a structure subjected to random earthquake ground motions, the five-storey elastic structure used for model verification in Section 5.2.1.2, and shown in Figure 5.2, was subjected to a sequence of 1000 generated records, using the El Centro ground motion as the seed, at five different levels of variability in the storey mass, storey stiffness, storey height and modal damping. To ensure that the frequency content of the generated records did not result in unrealistic ground displacements, the peak elastic displacement of a long period SDOF structure $(T=12.0$ seconds) was determined for each record in the sequence using; no filtering, filtering with a low frequency window transition of $0.05-0.075 \mathrm{~Hz}$ and filtering with a low frequency window transition of $0.1-$ $0.15 \mathrm{~Hz}$. In each case there was no significant difference in the statistics describing the distribution of the peak displacements. The variability levels of the structural properties were characterized by Normal distributions with a coefficient of variation (COV) of 0.0 , $0.1,0.2,0.3$ and 0.4 in each of the structural parameters with the exception of storey height where the COV's were set at $0.0,0.007,0.014,0.021$ and 0.028 , corresponding to standard deviations of 1 to 4 inches in the 12 foot storey height. The dynamic responses that were chosen for probabilistic analysis were limited to; peak base shear, peak base moment and peak fifth storey displacement.

Table 5.5 lists the basic statistics for each set of peak results that were generated using the same earthquake sequence as well as statistics for the peak results of the deterministic structure $(\mathrm{COV}=0.0)$ subjected to a second sequence of 1000 earthquakes.

The second earthquake sequence was used to verify that the probability distribution of each response quantity was not affected by a particular random sequence of earthquakes. For reference purposes, the peak elastic responses of the five-storey structure to the El 
Centro ground motion are bracketed in Table 5.5 below each response quantity in column one.

The actual frequency distributions corresponding to each set of calculated statistics in Table 5.5 are given as histogram plots in Figure 5.13 - Figure 5.15. These plots are overlaid with the associated Gumbel distribution, given as a solid line, and Normal distribution, given as a dashed line, to illustrate the fit of each type of distribution to the data. Note that the discontinuity in some of the Normal distributions is the result of lumping together all responses below the point of the discontinuity.

Table 5.5: Peak Response Statistics of a Five-Storey Elastic Shear Frame

\begin{tabular}{|c|c|c|c|c|c|c|c|}
\hline $\begin{array}{l}\text { Response } \\
\text { Quantity }\end{array}$ & Statistic & $\begin{array}{l}\text { Seq. } \# 1 \\
\mathrm{COV}=0.0\end{array}$ & $\begin{array}{c}\text { Seq } \# 2 \\
\mathrm{cOV}=0.0\end{array}$ & $\begin{array}{l}\text { Seq. } \# 1 \\
\mathrm{COV}=0.1\end{array}$ & $\begin{array}{c}S e g .11 \\
\operatorname{cov}=0.2\end{array}$ & $\begin{array}{c}\text { Seq. } \# 1 \\
\mathrm{COV}=0.3\end{array}$ & $\begin{array}{c}\text { Seq. } \# 1 \\
\mathrm{COV}=0.4\end{array}$ \\
\hline \multirow{4}{*}{$\begin{array}{l}\text { Base } \\
\text { Shear } \\
\text { (kips) } \\
(74.03)\end{array}$} & Min. & 27.40 & 28.16 & 31.96 & 27.99 & 19.79 & 13.67 \\
\hline & Max. & 155.35 & 185.68 & 173.67 & 193.67 & 169.14 & 290.82 \\
\hline & Avg. & 73.33 & 74.37 & 73.41 & 73.92 & 73.92 & 76.30 \\
\hline & Stdev. & 20.58 & 22.31 & 21.25 & 22.36 & 24.07 & 29.35 \\
\hline \multirow{4}{*}{$\begin{array}{l}\text { Base } \\
\text { Moment } \\
(\text { Kip*fi) } \\
(2587.53)\end{array}$} & $\mathrm{Mi}$ & 9940103 & 21153.60 & 988.00 & 945.33 & 832.28 & 493.37 \\
\hline & Max. & 6829.30 & 27128.50 & 6705.30 & 7882.40 & 6643.50 & 1224800 \\
\hline & A ve. & 284327 & 2878.58 & 2851.44 & 281901 & 2777.07 & 2718.96 \\
\hline & Stalev. & 877.92 & $\begin{array}{r}915.03 \\
\end{array}$ & 902.10 & 933.67 & 973.03 & 1133.05 \\
\hline \multirow{4}{*}{$\begin{array}{l}\text { Storey } 5 \\
\text { Displ. } \\
\text { (in.) } \\
(6.84)\end{array}$} & Min. & 2.63 & 3.05 & 2.66 & 2.75 & 2.69 & 2.59 \\
\hline & Max. & 18.04 & 18.83 & 19.21 & 21.11 & 28.70 & 37.46 \\
\hline & Avg. & 7.51 & 7.61 & 7.61 & 7.77 & 8.32 & 8.83 \\
\hline & Stdev. & 2.32 & 2.42 & 2.42 & 2.57 & 3.25 & 3.89 \\
\hline
\end{tabular}

From Figure 5.13, Figure 5.14 and Figure 5.15 it is apparent that the Gumbel distribution is a better description of the peak response data in each case than the Normal distribution, as expected, since the Gumbel distribution is an Extreme Value-Type I distribution. Therefore, using the Gumbel distribution as a basis for comparison, Figure 5.16 summarizes the individual distributions for each set of results to illustrate the effect of increasing variability in structural parameters on the probability distribution of the chosen elastic response quantities. 

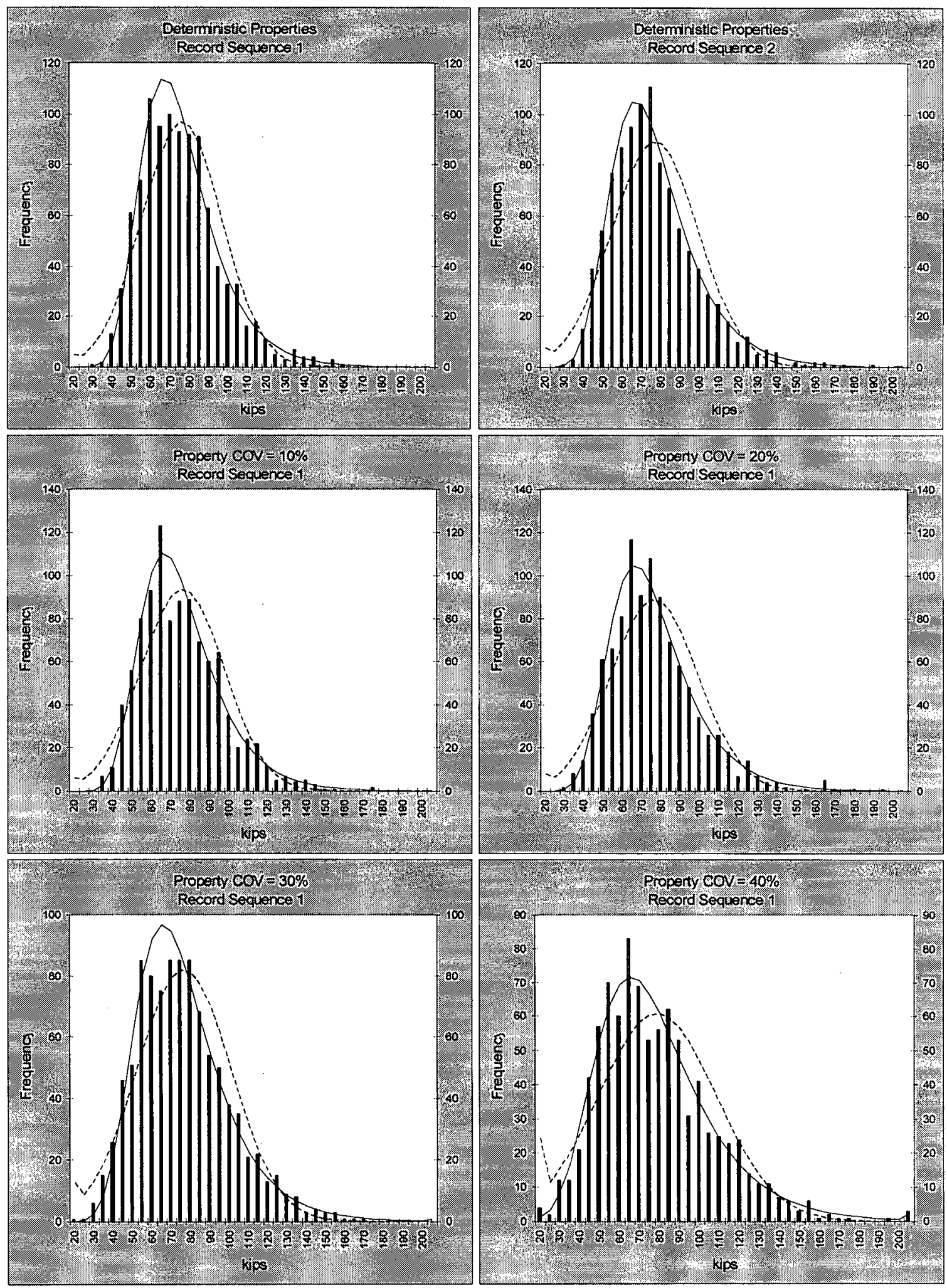

Figure 5.13: Base Shear Response Histograms 

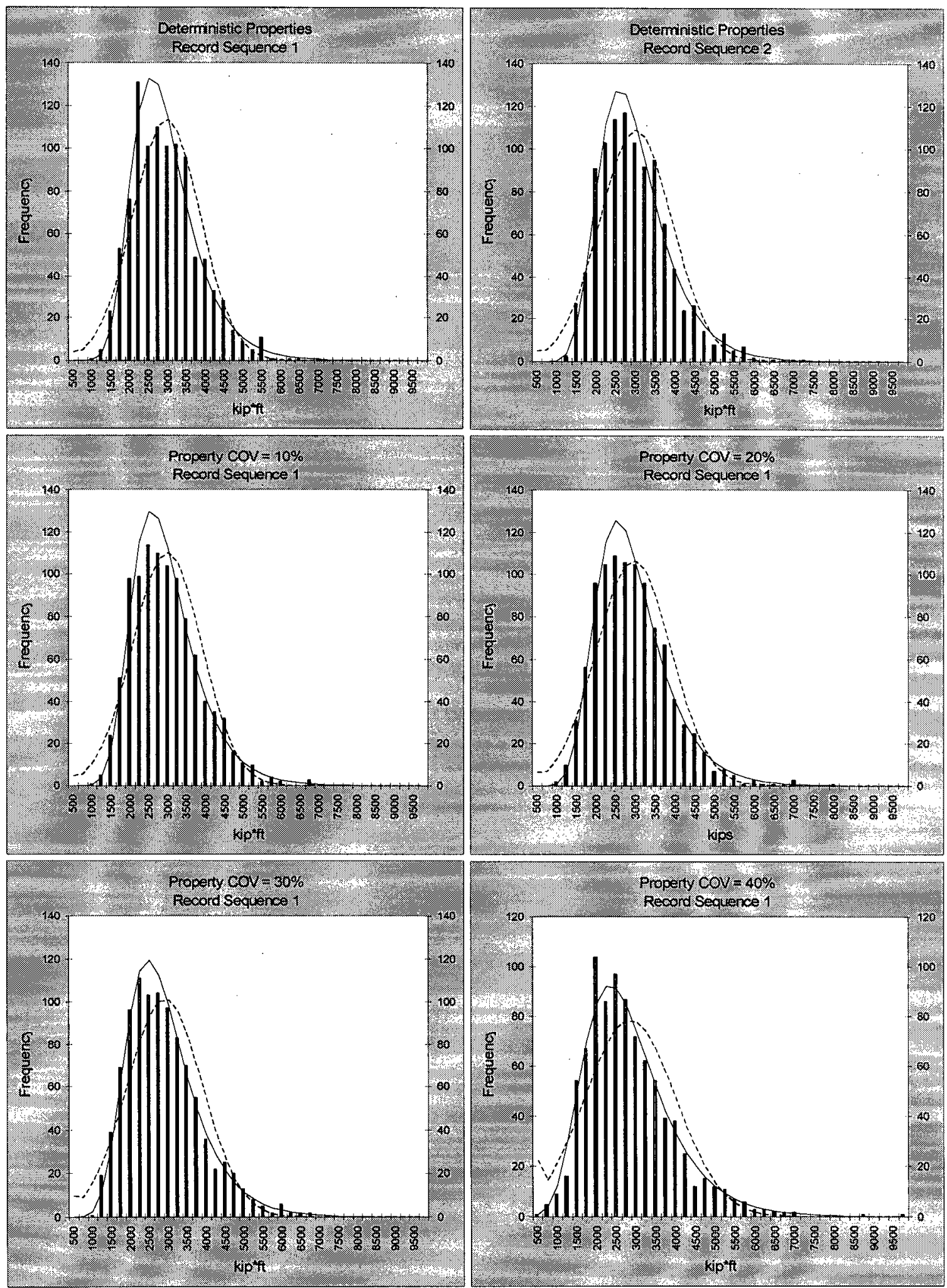

Figure 5.14: Base Moment Response Histograms 

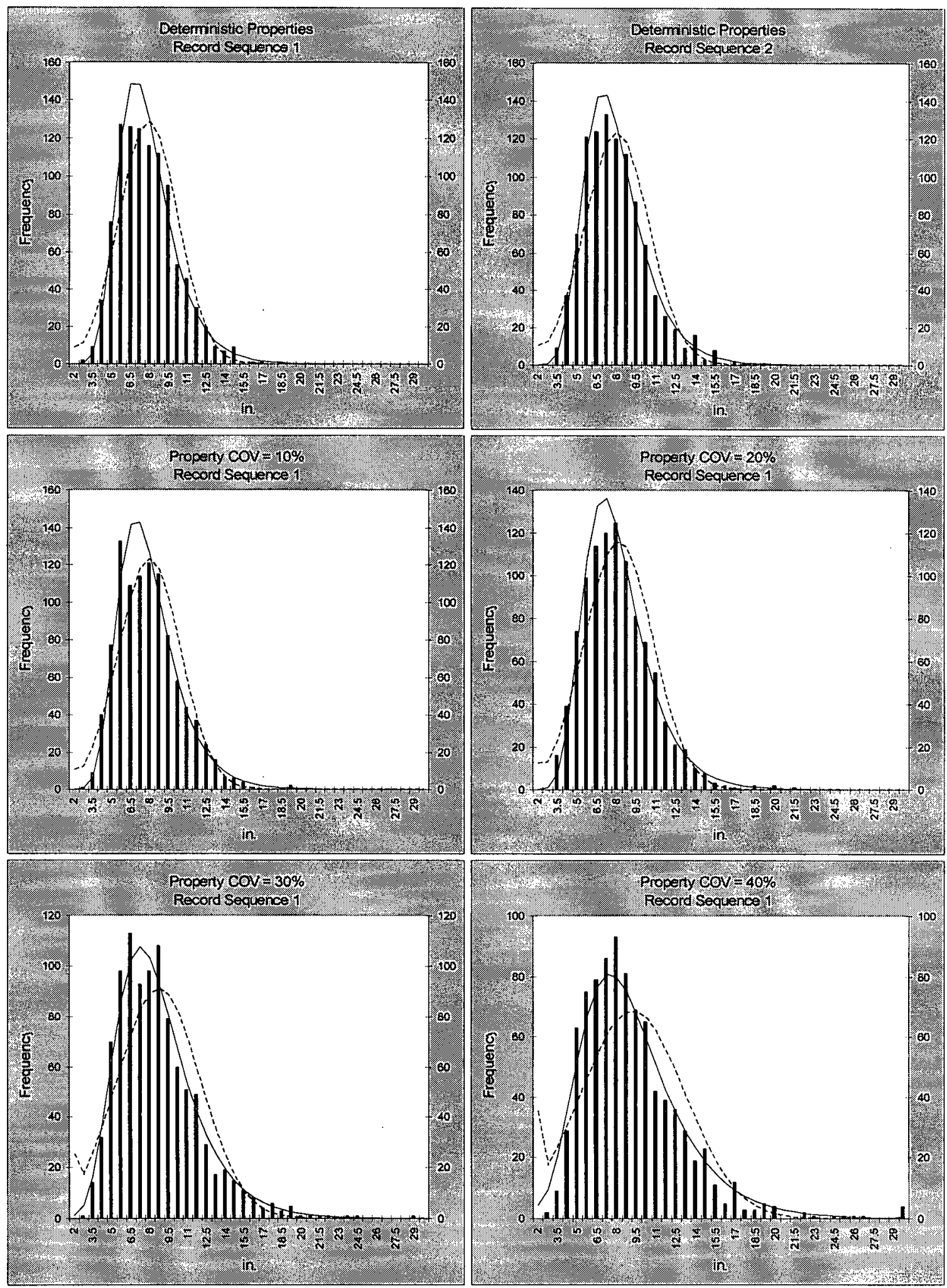

Figure 5.15: Storey 5 Displacement Response Histograms 

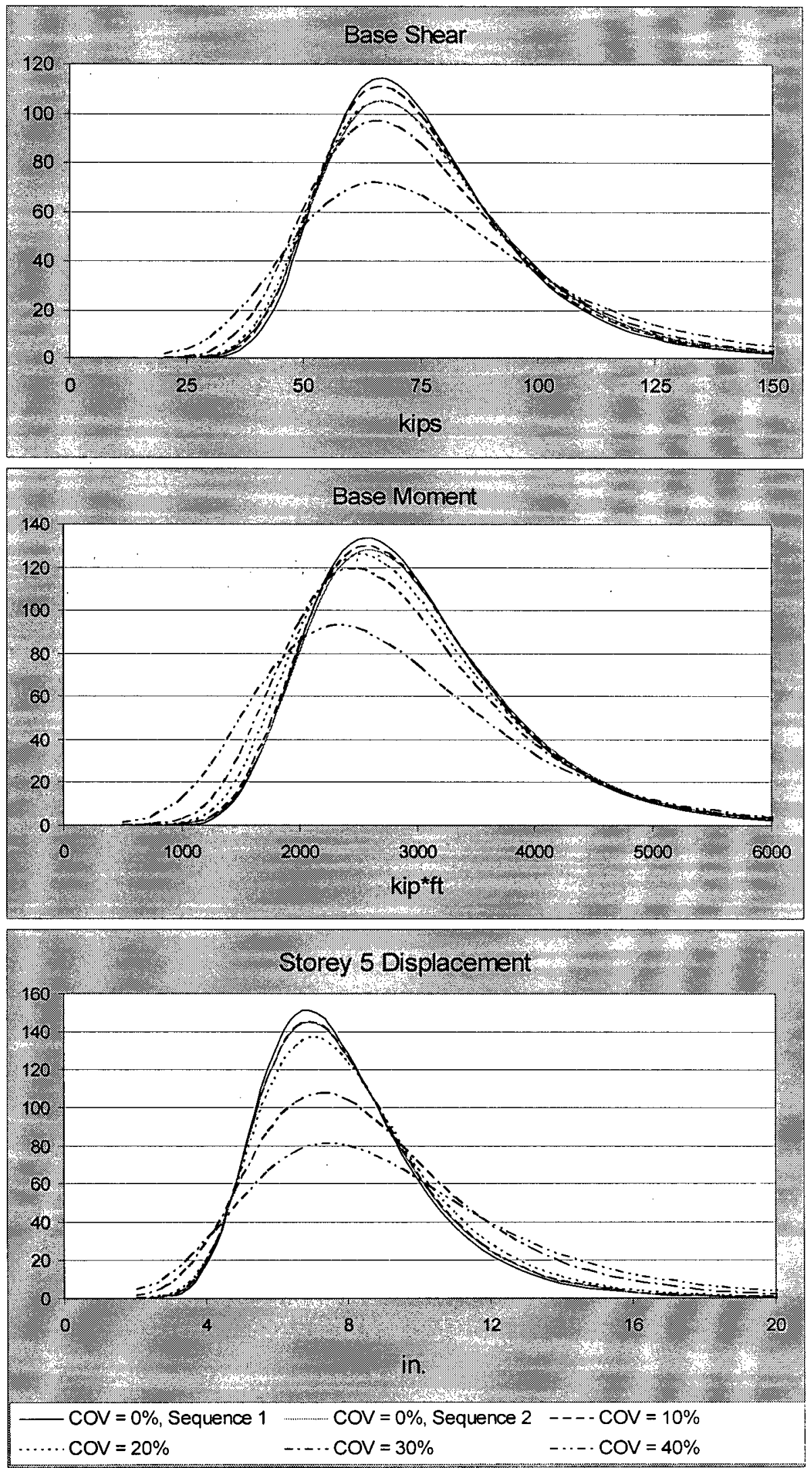

Figure 5.16: Peak Response Distributions 
To assess whether the individual distributions are statistically equivalent or significantly different, several statistical tests were performed to compare each set of peak results with those of the deterministic structure $(\mathrm{COV}=0.0)$. These tests evaluate whether two distributions possess the same mean (Student's t-test), and the same variance ( $F$-test) and, more generally, whether two distributions are equivalent based on the maximum difference between the cumulative distribution functions (KolmogorovSmirnov test). Table 5.6 lists the statistics comparing the response distribution for each level of structural variability with that of the deterministic structure, including the statistics comparing the response distributions of the deterministic structure using the two different generated record sequences.

Table 5.6: Elastic Peak Response Distribution Comparison Statistics

\begin{tabular}{|c|c|c|c|c|c|c|}
\hline $\begin{array}{l}\text { Response } \\
\text { Quantity }\end{array}$ & Statistic & $\begin{array}{c}\text { Seq. } \# 2 \\
C O V=0.0\end{array}$ & $\begin{array}{c}\text { Seg.\#1 } \\
\mathrm{cov}=0.1\end{array}$ & $\begin{array}{c}\text { Seq } \# 1 \\
\operatorname{cov}=0.2\end{array}$ & $\begin{array}{c}\text { Seq. } \# 1 \\
\text { COV }=0.3\end{array}$ & $\begin{array}{c}\text { Seq } 41 \\
\operatorname{COV}=0.4\end{array}$ \\
\hline \multirow{3}{*}{$\begin{array}{l}\text { Base Shear } \\
\text { (kips) }\end{array}$} & F & 1.1751 & 1.0659 & 1.1803 & 1.3681 & 2.0333 \\
\hline & $t$ & -1.0834 & -0.0890 & -0.6113 & -0.5891 & -2.5671 \\
\hline & $\overline{\mathrm{Q}_{\mathrm{KS}}}$ & 0.6785 & 0.6406 & 0.8840 & 0.0809 & 0.0003 \\
\hline \multirow{3}{*}{$\begin{array}{l}\text { Base } \\
\text { Moment } \\
\left(\mathrm{Kaip}^{*} \mathrm{ft}\right)\end{array}$} & $2 \mathrm{~F}:$ & 271.0863 & -1.0559 & 1.1311 & 1,2284 & 1.6657 \\
\hline & & . 0.8805 & -0.2051 & 0.5988 & 1.5939 & 2.6829 \\
\hline & Qks & $8 \times 0.7161$ & 0.8840 & 0.3344 & 00089 & $20.0000^{2}$ \\
\hline \multirow{3}{*}{$\begin{array}{l}\text { Storey } 5 \\
\text { Displ. } \\
\text { (in.) }\end{array}$} & $\mathrm{F}$ & 1.0863 & 1.0917 & 1.2236 & 1.9620 & 2.8190 \\
\hline & $\bar{t}$ & -0.8804 & -0.8978 & -2.3359 & -6.4176 & -9.0789 \\
\hline & $\mathrm{Q}_{\mathrm{KS}}$ & 0.7161 & 0.7888 & 0.0525 & 0.0000 & 0.0000 \\
\hline
\end{tabular}

Comparing the statistics of Table 5.6 with the critical values of $F(0.8496,1.1771)$ and $t(-2.57823,2.5783)$ at the $1 \%$ level of significance, it appears that a coefficient of variation of $10 \%$ in the storey mass, storey stiffness, storey height and modal damping does not significantly affect the elastic response distribution of peak base shear, peak base moment or peak fifth storey displacement for a random sequence of El Centro type earthquakes. At the $\mathrm{COV}=20 \%$ level of variability, the peak fifth storey displacement distribution was marginally affected while the peak base shear and peak base moment distributions remain unaffected. At the $\mathrm{COV}=30 \%$ level of variability, the peak fifth storey displacement distribution and the peak base shear distribution were both 
significantly affected while the peak base moment distribution was only marginally affected, however, this may be a result of the smaller variability used for storey height. At the $\mathrm{COV}=40 \%$ level of variability, all response distributions were significantly affected. These observations are supported by the Kolmogorov-Smirnoff test statistic $Q_{K S}$ for each set of peak responses, which may be interpreted as the probability that the underlying distribution of the peak responses is the same as that of the deterministic structure. In each case where the response distribution appears to be affected at a certain level of parameter variability, there is a corresponding sharp decrease in the $Q_{K S}$ statistic indicating that it is highly unlikely that the underlying distributions are equivalent. Lastly from Table 5.6, the distribution comparison statistics for the deterministic structure using the two different sequences of random earthquakes indicate that there is no significant difference in the distributions of any of the three types of peak responses. This suggests that the assumption that the probability distribution of each response quantity is not affected by a particular random sequence of earthquakes is valid.

Keeping in mind that only one particular type of earthquake and one basic pattern of storey mass, storey stiffness, storey height and modal damping were modeled, the foregoing results seem to indicate that the randomness of the generated ground motions accounts for the majority of the observed range in a given peak response while structural randomness has a relatively minor effect. This then suggests, or perhaps confirms the prevailing opinion, that careful attention needs to be paid to the characteristics of the ground motion records used when analyzing the dynamic response behaviour of a structure. Once a suitable seed record or suite of seed records has been selected, however, the peak response probability distributions for a given structural model could be applied to a real structure with reasonable confidence since the assumed level of uncertainty in the structural parameters needs to be only approximately correct.

Finally, to assess the accuracy of determining peak responses for a multipledegree-of-freedom system from a response spectrum analysis (RSA) procedure, the peak time-history (RHA) responses in each set of results were compared with modal combinations using three common modal combination rules; the absolute sum 
(ABSSUM) rule, the square-root-of-sum-of-squares (SRSS) rule and the complete quadratic combination (CQC) rule. Table 5.7 lists the basic statistics for each distribution of percentage differences between the RHA peak response and the corresponding RSA peak responses. For reference purposes, the percentage differences between the RHA and RSA results for the El Centro ground motion are bracketed in Table 5.7 below each modal combination type in column two.

Table 5.7: Statistics for the Percentage Difference between RHA \& RSA Results

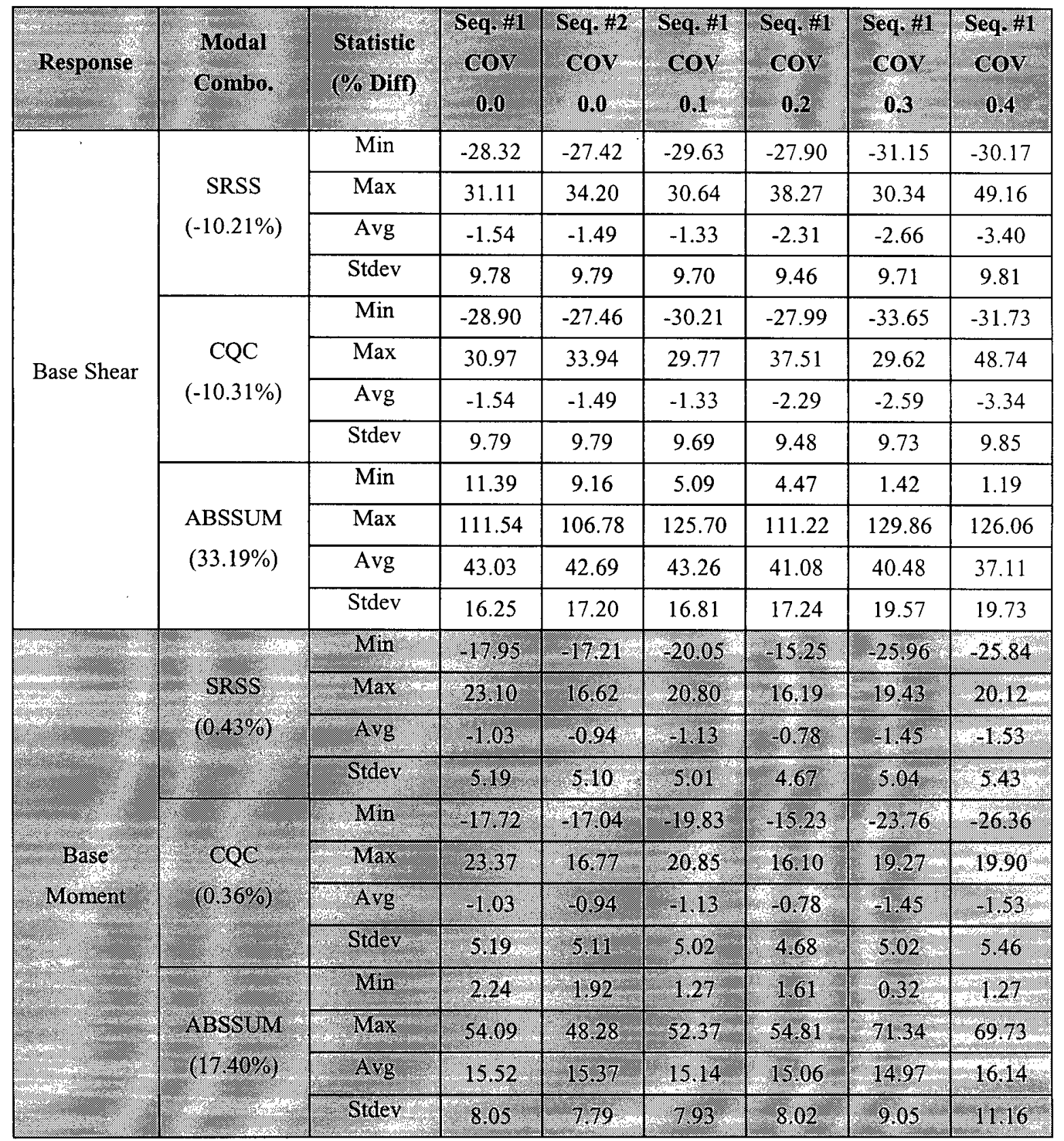




\begin{tabular}{|c|c|c|c|c|c|c|c|c|}
\hline Response & $\begin{array}{l}\text { Modal } \\
\text { Combo. }\end{array}$ & $\begin{array}{l}\text { Statistic } \\
\text { (\% Difn) }\end{array}$ & $\begin{array}{c}\mathrm{Seq} \cdot \mathrm{fl}^{-1} \\
\mathrm{COV} \\
0.0\end{array}$ & $\begin{array}{c}\text { Seq. \#2 } \\
\text { COV } \\
0.0\end{array}$ & $\begin{array}{c}\text { Seq. } \# 1 \\
\text { cov } \\
0.1\end{array}$ & $\begin{array}{c}\text { Seq. } \# 1 \\
\text { Cov } \\
0.2\end{array}$ & $\begin{array}{c}\text { Seq. }+1 \\
\text { cov } \\
0.3\end{array}$ & $\begin{array}{c}\text { Seq. }+1 \\
\text { Cov } \\
0.4\end{array}$ \\
\hline \multirow{12}{*}{$\begin{array}{c}\text { Storey } 5 \\
\text { Displ. }\end{array}$} & \multirow{4}{*}{$\begin{array}{c}\text { SRSS } \\
(0.44 \%)\end{array}$} & Min & -17.95 & -17.21 & -18.06 & -20.86 & -22.49 & -18.88 \\
\hline & & Max & 23.11 & 16.62 & 21.04 & 16.22 & 16.66 & 18.48 \\
\hline & & Avg & -1.03 & -0.94 & -1.11 & -0.92 & -1.12 & -0.91 \\
\hline & & $\overline{\text { Stdev }}$ & 5.19 & 5.10 & 5.01 & 4.81 & 4.72 & 4.66 \\
\hline & \multirow{4}{*}{$\begin{array}{c}\text { CQC } \\
(0.37 \%)\end{array}$} & Min & -17.72 & -17.04 & -17.87 & -21.06 & -22.01 & -18.68 \\
\hline & & Max & 23.37 & 16.77 & 21.11 & 16.09 & 16.39 & 18.16 \\
\hline & & Avg & -1.03 & -0.94 & -1.11 & -0.92 & -1.12 & -0.91 \\
\hline & & Stdev & 5.19 & 5.11 & 5.01 & 4.83 & 4.71 & 4.65 \\
\hline & \multirow{4}{*}{$\begin{array}{c}\text { ABSSUM } \\
(17.41 \%)\end{array}$} & Min & 2.24 & 1.92 & 1.20 & 2.24 & 0.84 & 0.87 \\
\hline & & $\overline{\text { Max }}$ & 54.09 & 48.28 & 55.12 & 48.39 & 53.13 & 54.43 \\
\hline & & Avg & 15.52 & 15.37 & 15.22 & 14.78 & 13.60 & 12.91 \\
\hline & & Stdev & 8.05 & 7.79 & 7.84 & 7.62 & 7.87 & 8.46 \\
\hline
\end{tabular}

Comparing any distribution statistic in Table 5.7 across a row shows that the statistic remains approximately constant at each level of structural variability. This was not unexpected since the modal combination rules are not direct functions of any type of uncertainty in the structural parameters, rather they are functions of individual peak modal responses, and in the case of the CQC rule, a correlation coefficient that depends only on modal frequencies and damping. Therefore, given a random structure, the associated natural frequencies and random damping determine both the peak RHA response and RSA results for a given earthquake motion without regard to the structural variability that generated the random structure. Although not directly influenced by structural variability, the accuracy of each set of RSA results is certainly affected by the variation in each random structure but in a manner that is difficult to predict. Each unique set of natural frequencies and modal damping results in a different relationship between the magnitudes of the peak modal responses and, therefore, a different level of influence of the higher modes of response, which in turn has a direct effect on the accuracy of the RSA results. For a certain random structural realization in which the fundamental mode dominates the total response, the RSA results will tend to be quite accurate whereas another structural realization with more significant higher modes of 
response will tend to show larger errors in the RSA results. The difficulty in predicting the effect of a certain level of structural randomness on RSA accuracy is due to the jagged nature of the response spectrum of a single earthquake. Individual modal responses may be negligibly or significantly affected by large or small random variations in modal frequencies and damping, particularly at certain periods, which results in an unpredictable effect on the modal combination and hence an unpredictable effect on RSA accuracy. Furthermore, the relationship between the accuracy of the CQC results as compared to the SRSS and ABSSUM results is influenced by the degree of separation between the natural frequencies for a given structure. Structures with well-separated natural frequencies will exhibit little or no difference between CQC and SRSS peak responses with the SRSS peak response becoming relatively less accurate for more closely spaced natural frequencies.

The observed distribution statistics in Table 5.7 for the ABSSUM results, which are an upper-bound on the peak response, show an average overestimation of the peak base shear of approximately $42 \%$, and an average overestimation of the peak base moment and peak fifth storey displacement of approximately 15\%. Clearly, the ABSSUM modal combination rule gives a very conservative estimate of peak response. The SRSS and CQC results for each response type across the range of structural variability were virtually identical, which indicates that the natural frequencies of the five-storey shear frame were well-separated at each level of variability. The average error for the peak base shear was in the range of $-1.3 \%$ to $-3.4 \%$, the average error for the peak base moment was in the range of $-0.8 \%$ to $-1.5 \%$, and the average error for the peak fifth storey displacement was in the range of $-0.9 \%$ to $-1.1 \%$. The error is largest for the peak base shear because the higher mode responses are likely more significant relative to the first mode as compared to the peak base moment and peak fifth storey displacement (see Table 5.2). Although the SRSS and CQC peak responses consistently underestimated the RHA peak responses in this limited study, this is not a general trend for response spectrum analysis. The variance in each set of errors was observed, as noted previously, to be approximately constant across the range of structural variability in Table 5.7, therefore, it is well represented for each response quantity by the results for the 
deterministic structure. Figure 5.17 shows the histogram plots for the SRSS and CQC errors in peak base shear, peak base moment and peak fifth storey displacement. Included with the histogram plots are the associated Normal distributions to illustrate the close agreement with the observed error distribution.

Using the Normal distributions in Figure 5.17 to represent the SRSS and CQC error distribution, the variance or standard deviation for each response quantity is a measure of the level of confidence that may be applied to an estimate of the accuracy of each modal combination rule. From the basic properties of a Normal distribution, $68.3 \%$ of the area is contained within one standard deviation of the mean, which indicates that approximately $2 / 3$ of observed results should be no more or no less than one standard deviation different from the average value. Using this property, approximate rules-ofthumb can be established for the accuracy of the CQC and SRSS modal combination rules for peak base shear, peak base moment and peak fifth storey displacement. Taking the mean errors in Table 5.7 to be approximately zero, the observed standard deviations indicate that $2 / 3$ of the time the CQC and SRSS results for peak base shear will be within $10 \%$ of the RHA result, while peak base moment and peak fifth storey displacement will be within $5 \%$ of the RHA result.

The preceding approximate rules-of-thumb and observations regarding the relative effect of structural variability on peak response distribution were, of course, derived from a very limited study using only one particular type of earthquake and one basic pattern of storey mass, storey stiffness, storey height and modal damping. However, they serve as an example of the type of analysis that may be done using PSResponse to provide fast, summary-level dynamic analysis results for determining the probabilistic response of a structure under stochastic dynamic loading. 

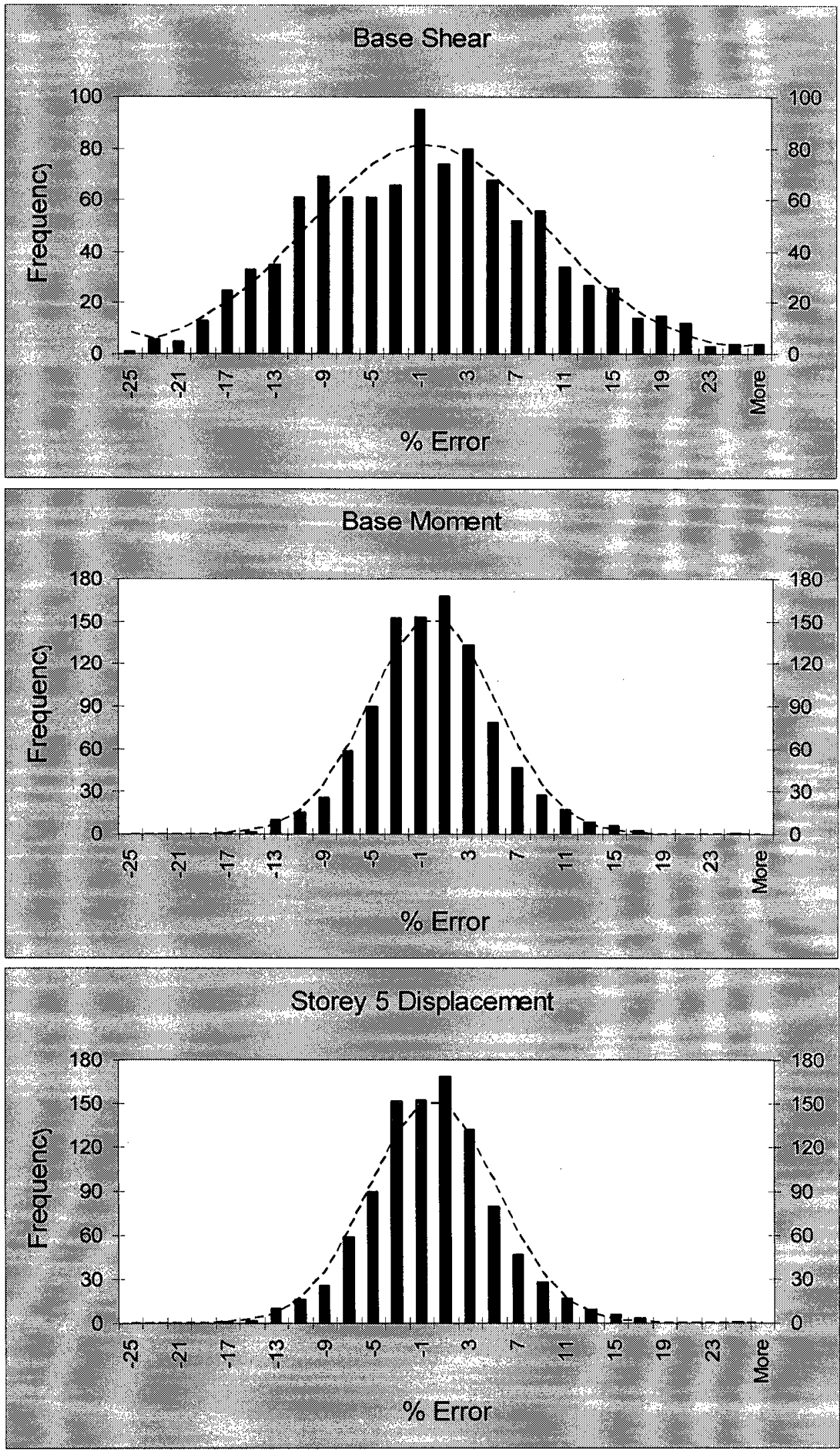

Figure 5.17: Error Distribution for CQC \& SRSS vs. RHA Peak Responses 


\subsubsection{Case Study \#2}

To assess the appropriateness of the well-known equal displacement observation in structural dynamics and the effect of hysteresis model properties on displacement response, a group of single-degree-of-freedom structures with eight different natural periods and $2 \%$ damping were subjected to a sequence of 1000 generated records, again using the El Centro ground motion as the seed, using six different hysteresis models. The natural periods of the structures were selected from the deformation and acceleration spectra for the El Centro earthquake to cover a reasonably wide range of periods that might be encountered in an actual structure. The periods that were selected were; 0.1 , $0.2,0.4,0.5,0.6,1.0,3.0$ and 8.0 seconds, which are shown on the response spectra in Figure 5.18. The hysteresis models were derived from experimental data taken from a cyclic lateral displacement-controlled test of a Parallam ${ }^{\circledR}$ column, which was connected to a rigid base with special hollow steel tubes acting as dowels (Ruxton 2003). The cyclic test data was analyzed using the hysteresis parameter identification feature of PSResponse, which identifies the best hysteresis parameter set based on a least-squares error algorithm. The displacement and force time-histories of the cyclic test as well as the experimental hysteresis loop and corresponding best--fit model are shown in Figure 5.19 .
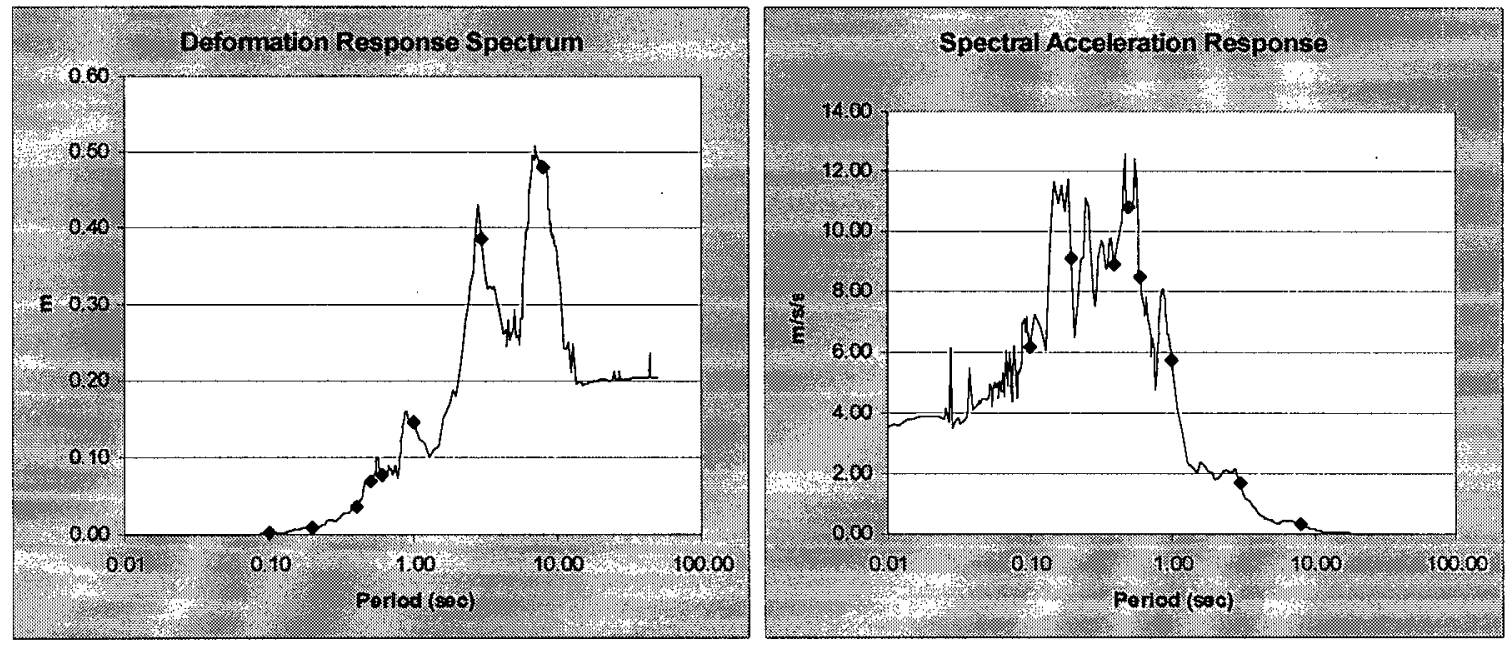

Figure 5.18: El Centro Response Spectra 

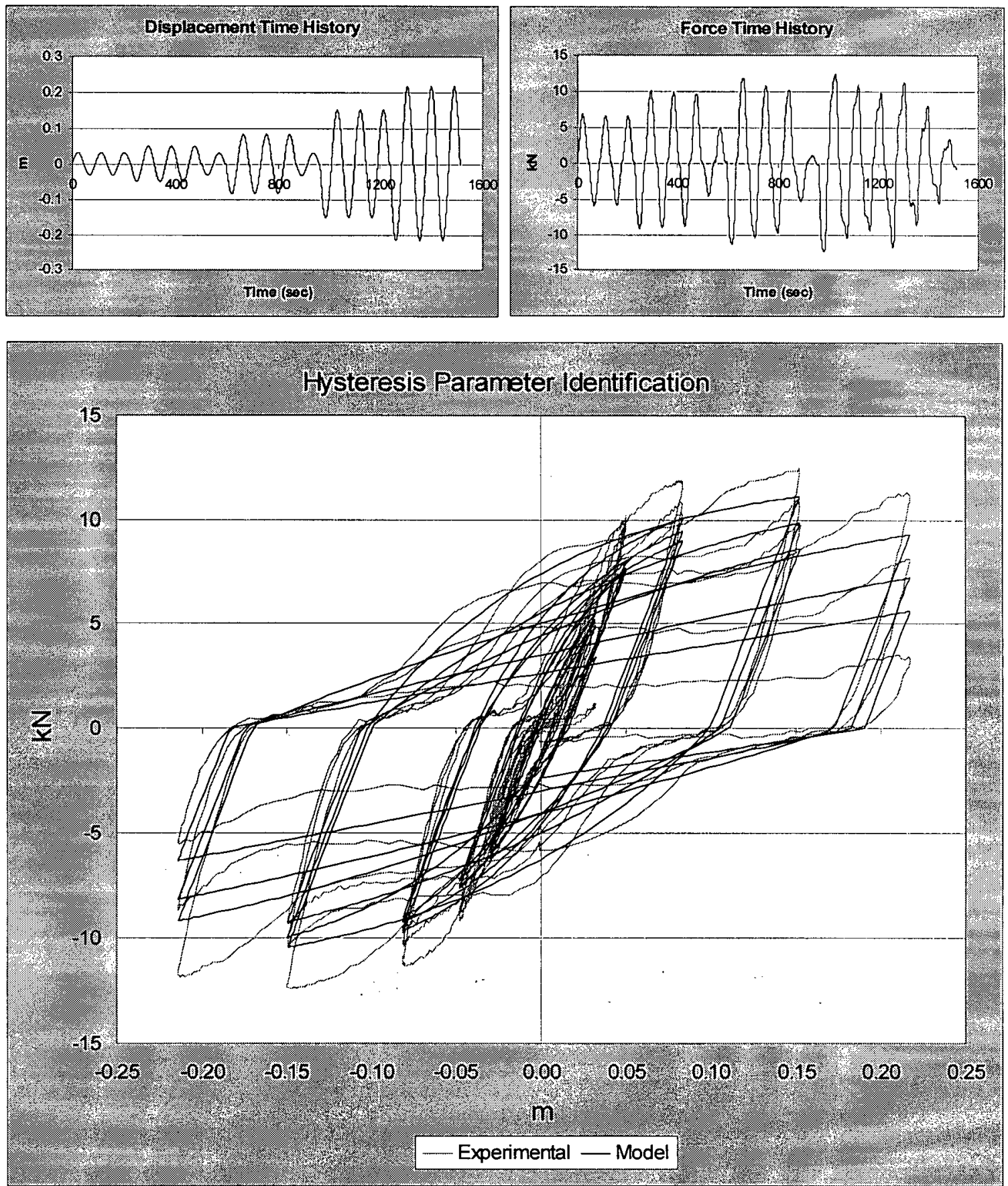

Figure 5.19: Cyclic Test of Parallam ${ }^{\circledR}$ Column and Best-Fit Hysteresis Model

Note from Figure 5.19 that the best-fit model is a compromise between two regions of pinching and stiffness recovery in one direction of loading for the large amplitude displacement cycles. The large displacement cycles begin as usual with a 
pinched region at the beginning of the loading cycle followed by stiffness recovery and a stiffness plateau but this is followed by another region of increasing stiffness in the same half-cycle of loading. This unusual behaviour does not fit well with the behaviour of the modified BWBN hysteresis model that was incorporated into PSResponse, therefore, the hysteresis parameter identification algorithm identified the best-fit compromise between the two regions of stiffness recovery.

The hysteresis models that were derived from the best-fit model of Figure 5.19 are listed in Table 5.8. These models were chosen to represent a broad range of possible inelastic behaviour exhibited by structures with different yield strengths and rates of structural deterioration. The different characteristics of each model were achieved by simply altering the appropriate best-fit hysteresis parameters that had been identified by PSResponse. To illustrate the behaviour of each hysteresis model, Figure 5.20 shows the first five seconds of deformation response to the El Centro ground motion for a singledegree-of-freedom structure with $\mathrm{T}=8.0$ seconds and $2 \%$ damping for each of the six models.

Table 5.8: Hysteresis Model Descriptions

\begin{tabular}{|c|c|c|}
\hline Model & 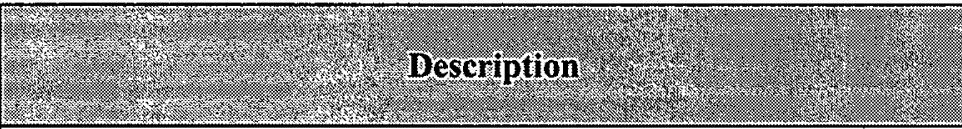 & $\begin{array}{l}\text { Pield } \\
\text { Displacement } \\
(\mathrm{m})\end{array}$ \\
\hline A & Best-fit hysteresis model from test data. & 0.060 \\
\hline $\bar{B}$ & Best-fit hysteresis model with degradation parameters doubled. & 0.060 \\
\hline $\mathrm{C}$ & Best-fit hysteresis model with degradation parameters quadrupled. & 0.060 \\
\hline $\mathrm{D}$ & No degradation $-100 \%$ yield strength of Model A. & 0.060 \\
\hline $\bar{E}$ & No degradation $-50 \%$ yield strength of Model $\mathrm{A}$. & 0.030 \\
\hline $\mathrm{F}$ & No degradation $-25 \%$ yield strength of Model $\mathrm{A}$. & 0.015 \\
\hline
\end{tabular}

Having established a number of hysteresis models to simulate a range of inelastic behaviour, the deformation response of a single-degree-of-freedom structure was determined at the eight natural periods noted previously using the same sequence of 1000 generated earthquake records for each period and hysteresis model combination. Table 
5.9 lists the basic statistics for each set of peak deformation results as well as the statistics for the peak deformation of an elastic structure at each period.

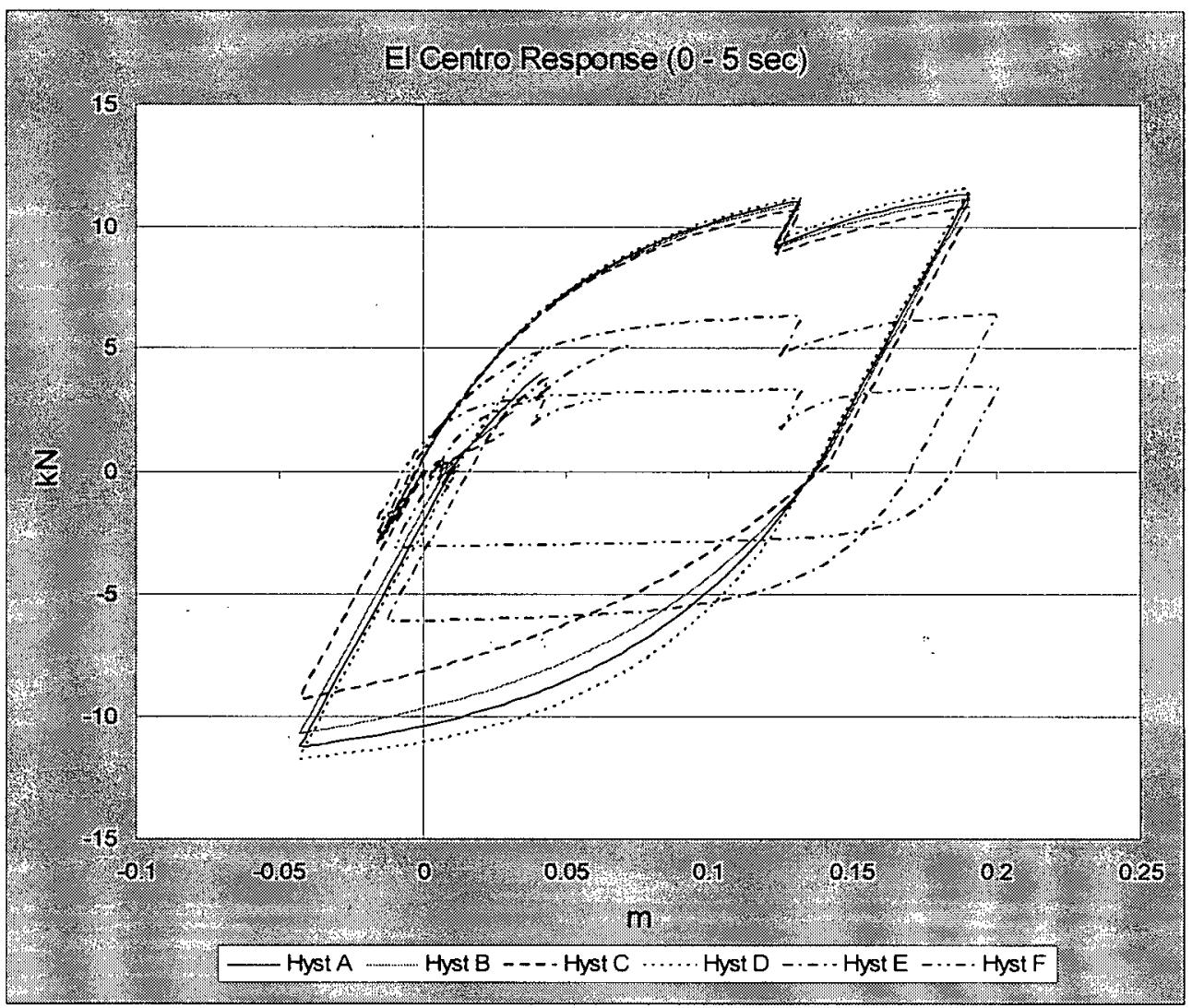

Figure 5.20: Hysteretic Response of SDOF Structure with $T=8.0 \mathrm{sec}$, Damping $=\mathbf{2} \%$

Table 5.9: SDOF Peak Deformation Response Statistics (m)

\begin{tabular}{|c|c|c|c|c|c|c|c|c|}
\hline \multirow{2}{*}{ T } & Statistic & Flastic & Hyst A & Hyst B & Hyst C & Hyst D & Hyst E & Hyst F \\
\hline \multirow{3}{*}{$\begin{array}{c}0.1 \\
\text { sec. }\end{array}$} & Min. & 0.00083 & 0.00082 & 0.00082 & 0.00082 & 0.00082 & 0.00084 & 0.00082 \\
\cline { 2 - 9 } & Max. & 0.00236 & 0.00225 & 0.00225 & 0.00227 & 0.00222 & 0.00223 & 0.00220 \\
\cline { 2 - 9 } & Avg. & 0.00141 & 0.00140 & 0.00140 & 0.00140 & 0.00140 & 0.00140 & 0.00140 \\
\cline { 2 - 9 } & Stdev. & 0.00024 & 0.00023 & 0.00023 & 0.00023 & 0.00023 & 0.00023 & 0.00023 \\
\hline \multirow{2}{*}{0.2} & Min. & 0.00457 & 0.00461 & 0.00461 & 0.00462 & 0.00460 & 0.00460 & 0.00427 \\
\hline \multirow{2}{*}{ sec. } & Max. & 0.02312 & 0.01526 & 0.01557 & 0.01434 & 0.01656 & 0.01447 & 0.01469 \\
\hline & Avg. & 0.01012 & 0.00883 & 0.00877 & 0.00864 & 0.00894 & 0.00841 & 0.00796 \\
\hline
\end{tabular}




\begin{tabular}{|c|c|c|c|c|c|c|c|c|}
\hline T & Statistic & Blastic & Hyst A & Hyst B & Fyst C & Myst D & Hyst E & Hyst 1 \\
\hline \multirow{4}{*}{$\begin{array}{l}0.4 \\
\text { sec. }\end{array}$} & Min. & 0.01784 & 0.01439 & 0.01438 & 0.01400 & 0.01468 & 0.01324 & 0.01206 \\
\hline & Max. & 0.08166 & 0.05231 & 0.05515 & 0.05547 & 0.05180 & 0.04943 & 0.06213 \\
\hline & Avg. & 0.03709 & 0.02910 & 0.02910 & 0.02932 & 0.02919 & 0.02842 & 0.03114 \\
\hline & Stddey & 0.00928 & 0.00539 & 0.00544 & 0.00556 & 0.00540 & 0.00529 & 0.00722 \\
\hline \multirow{4}{*}{$\begin{array}{c}0.5 \\
\mathrm{sec}\end{array}$} & & 0002416 & 0.02116 & 20.02100 & 0.02033 & 0.02116 & 0.01919 & 0.01882 \\
\hline & Max. & 0.11331 & 0.06816 & 0.06832 & 0.07154 & 0.06709 & 0.07387 & 0.09819 \\
\hline & & 0.05788 & 0.041411 & 004138 & 0.04151 & 0.041149 & 0.04033 & 0.04548 \\
\hline & Staey: & 00.01540 & 0.00772 & 0.00783 & 0.00824 & 0.00758 & 0.00814 & 0.01251 \\
\hline \multirow{4}{*}{$\begin{array}{l}0.6 \\
\text { sec. }\end{array}$} & Min. & 0.03165 & 0.02344 & 0.02300 & 0.02343 & 0.02462 & 0.02462 & 0.02226 \\
\hline & Max. & 0.15964 & 0.09256 & 0.09318 & 0.10498 & 0.09295 & 0.10506 & 0.13715 \\
\hline & $\overline{\mathrm{Avg}}$ & 0.07457 & 0.05124 & 0.05121 & 0.05153 & 0.05128 & 0.05090 & 0.05841 \\
\hline & $\mathrm{St}$ & 0.02054 & 0.01039 & 0.01063 & 0.01136 & 0.01023 & 0.01238 & 0.01824 \\
\hline \multirow{4}{*}{$\begin{array}{r}1,0 \\
\text { sec } \\
\end{array}$} & & 0.03881 & 0.03608 & 0.03641 & 0.03541 & 0.03559 & 0.03524 & 0.03503 \\
\hline & & 0.25019 & 0.15670 & 0.16267 & 0.17988 & 0.15322 & 0.21269 & 0.34074 \\
\hline & Avg. & 0.11296 & 0.07759 & 0.07748 & 0.07848 & 007799 & 0.08316 & 0.10252 \\
\hline & Stdey & 0.03412 & 0.01984 & 0.02001 & 0.02139 & 0.01994 & 0.02628 & 0.03952 \\
\hline \multirow{4}{*}{$\begin{array}{l}3.0 \\
\text { sec. }\end{array}$} & Min. & 0.06733 & 0.05492 & 0.05501 & 0.05435 & 0.05706 & 0.06479 & 0.07339 \\
\hline & Max. & 1.11230 & 0.81400 & 0.82257 & 0.82161 & 0.82328 & 1.05070 & 1.28130 \\
\hline & Avg. & 0.34202 & 0.21224 & 0.21426 & 0.22266 & 0.21450 & 0.25422 & 0.30196 \\
\hline & $\bar{S}$ & 0.15300 & 0.09276 & 0.09787 & 0.10225 & 0.09410 & 0.12333 & 0.15232 \\
\hline \multirow{4}{*}{$\begin{array}{l}8.0 \\
\text { sec }\end{array}$} & Min. & 0.09298 & 0.07416 & 0.07930 & 0.07801 & 0.07947 & 0.08089 & 0.07906 \\
\hline & Max. & 1.30990 & 2.53050 & 2.531110 & 3.04320 & 2.51870 & 3.24960 & 372100 \\
\hline & Avg & 0.42486 & 0.43666 & 0.45300 & 0.50538 & 0.43297 & 0.56794 & 0.71517 \\
\hline & Stder. & 0.20187 & 0.28548 & 0.30441 & 0.34712 & 0.28002 & 0.39477 & 0.50414 \\
\hline
\end{tabular}

The actual frequency distributions corresponding to each set of calculated statistics in Table 5.9 are given as histogram plots in Figure 5.21 - Figure 5.28. These plots are once again overlaid with the associated Gumbel distribution, given as a solid line, and Normal distribution, given as a dashed line, to illustrate the fit of each type of distribution to the data. Again note that the discontinuity in some of the Normal distributions is the result of lumping together all responses below the point of the discontinuity. 

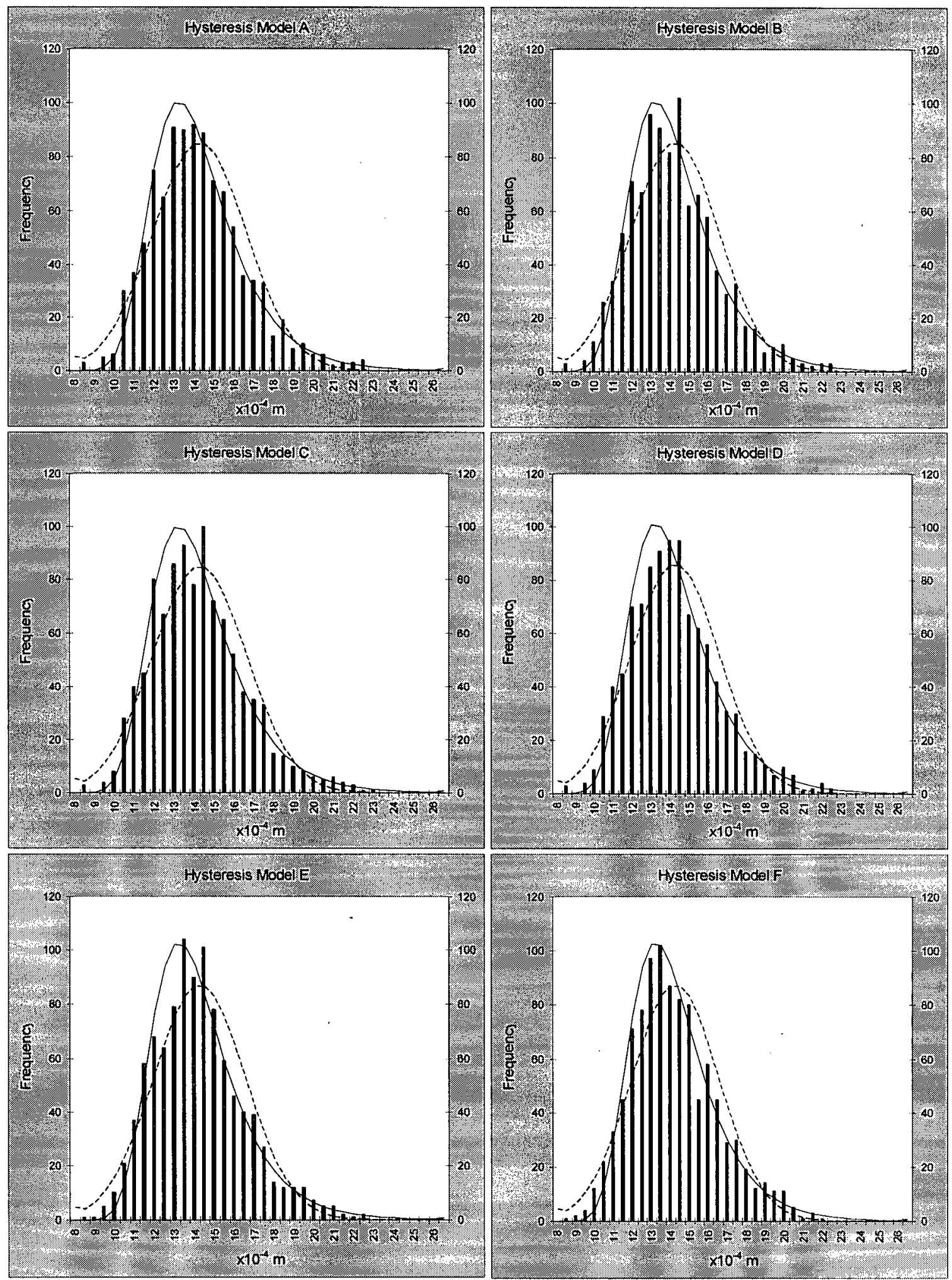

Figure 5.21: Displacement Response Histograms, $T=0.1 \mathrm{sec}$, Damping $=2 \%$ 

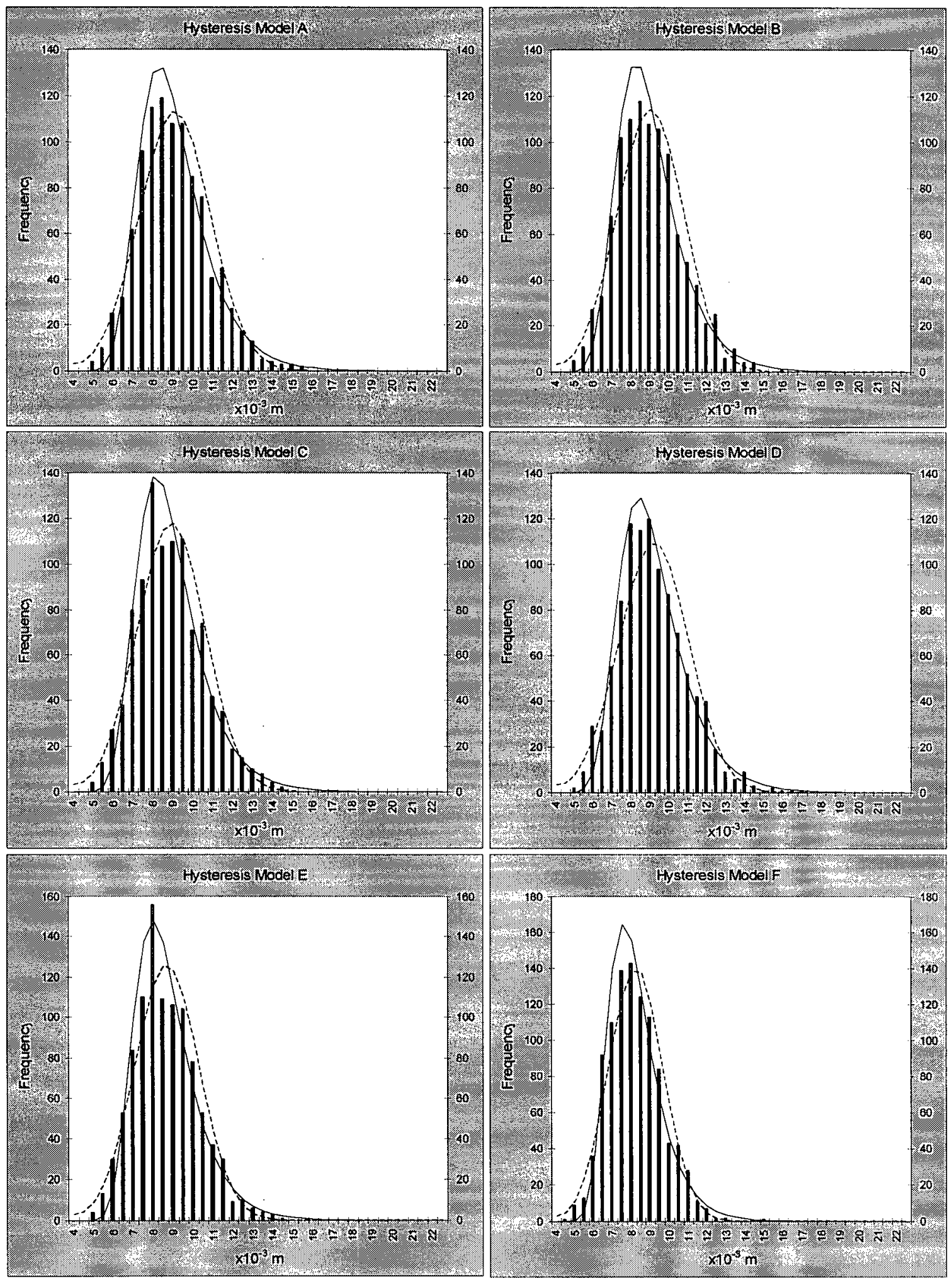

Figure 5.22: Displacement Response Histograms, $T=0.2 \mathrm{sec}$, Damping $=2 \%$ 

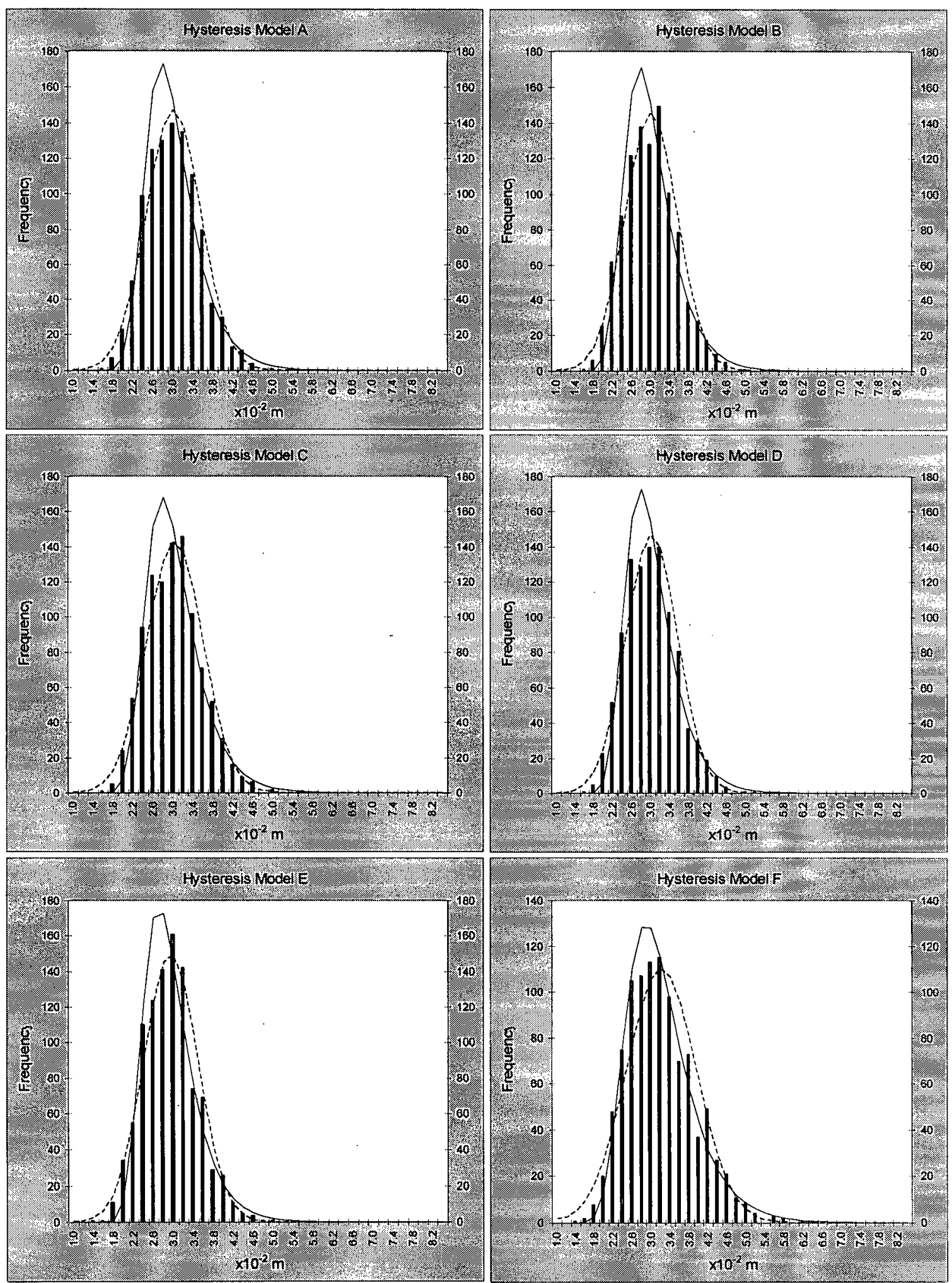

Figure 5.23: Displacement Response Histograms, $T=0.4$ sec, Damping $=2 \%$ 

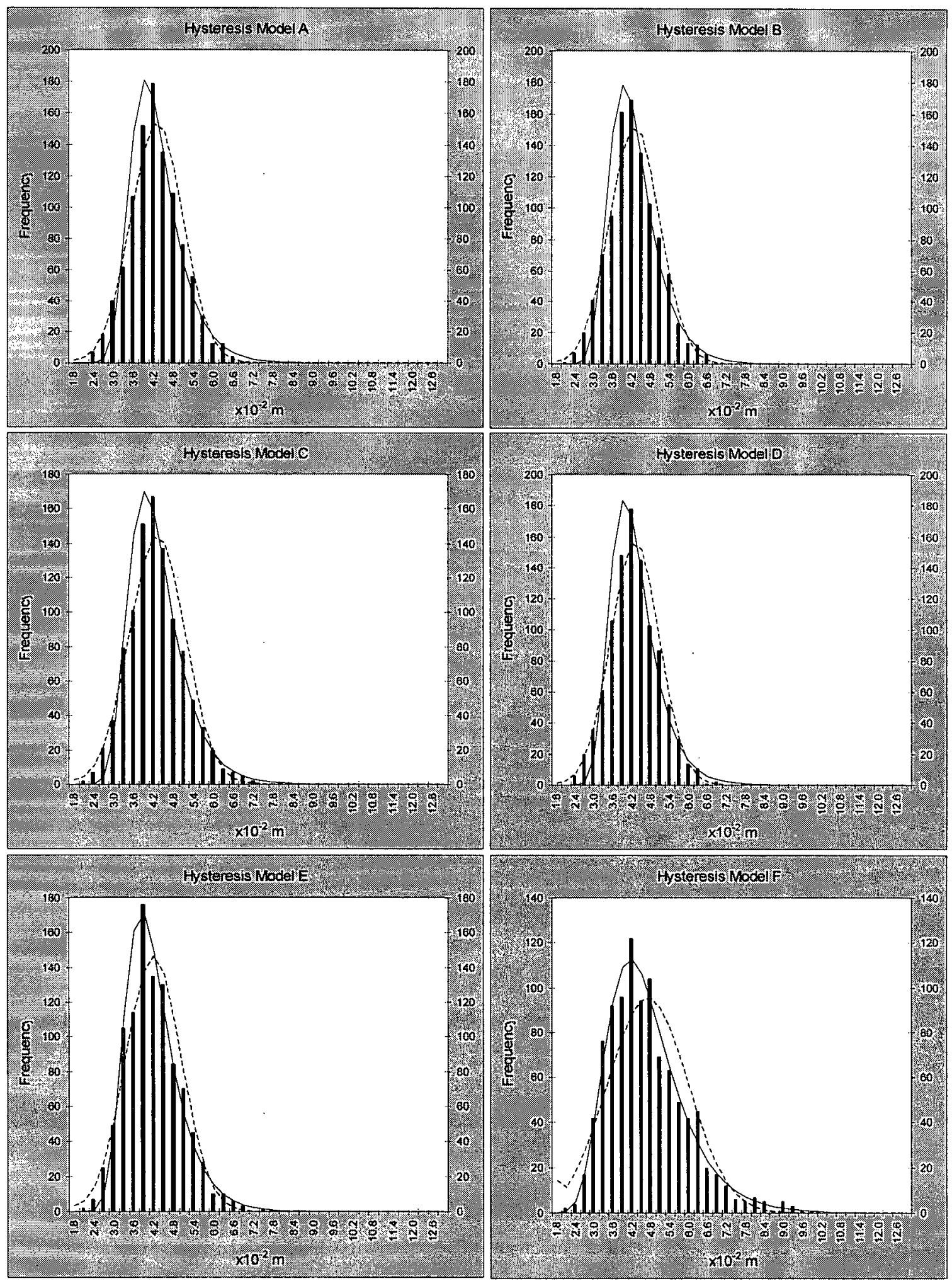

Figure 5.24: Displacement Response Histograms, $T=0.5$ sec, Damping $=2 \%$ 

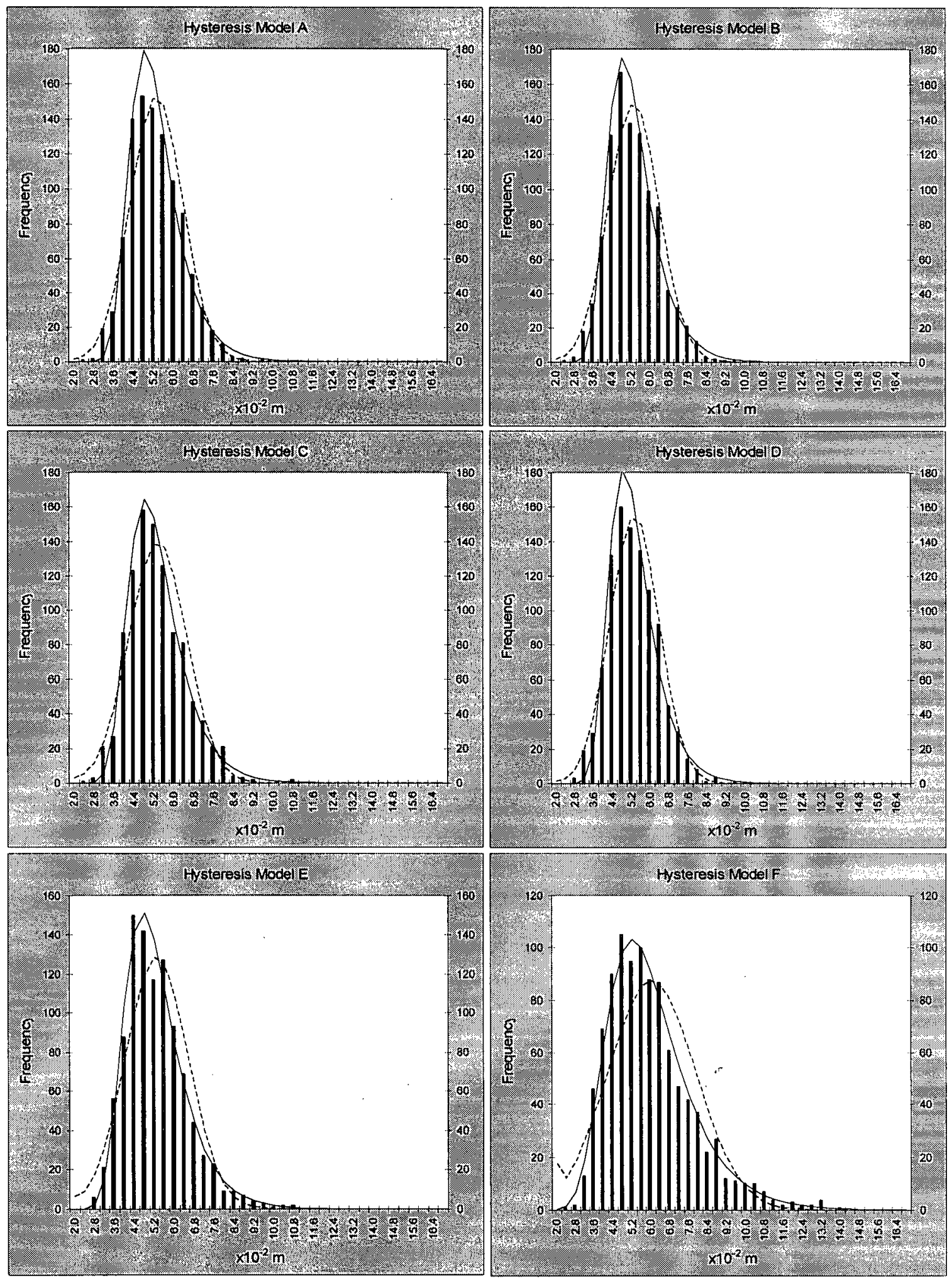

Figure 5.25: Displacement Response Histograms, $T=0.6 \mathrm{sec}$, Damping $=2 \%$ 

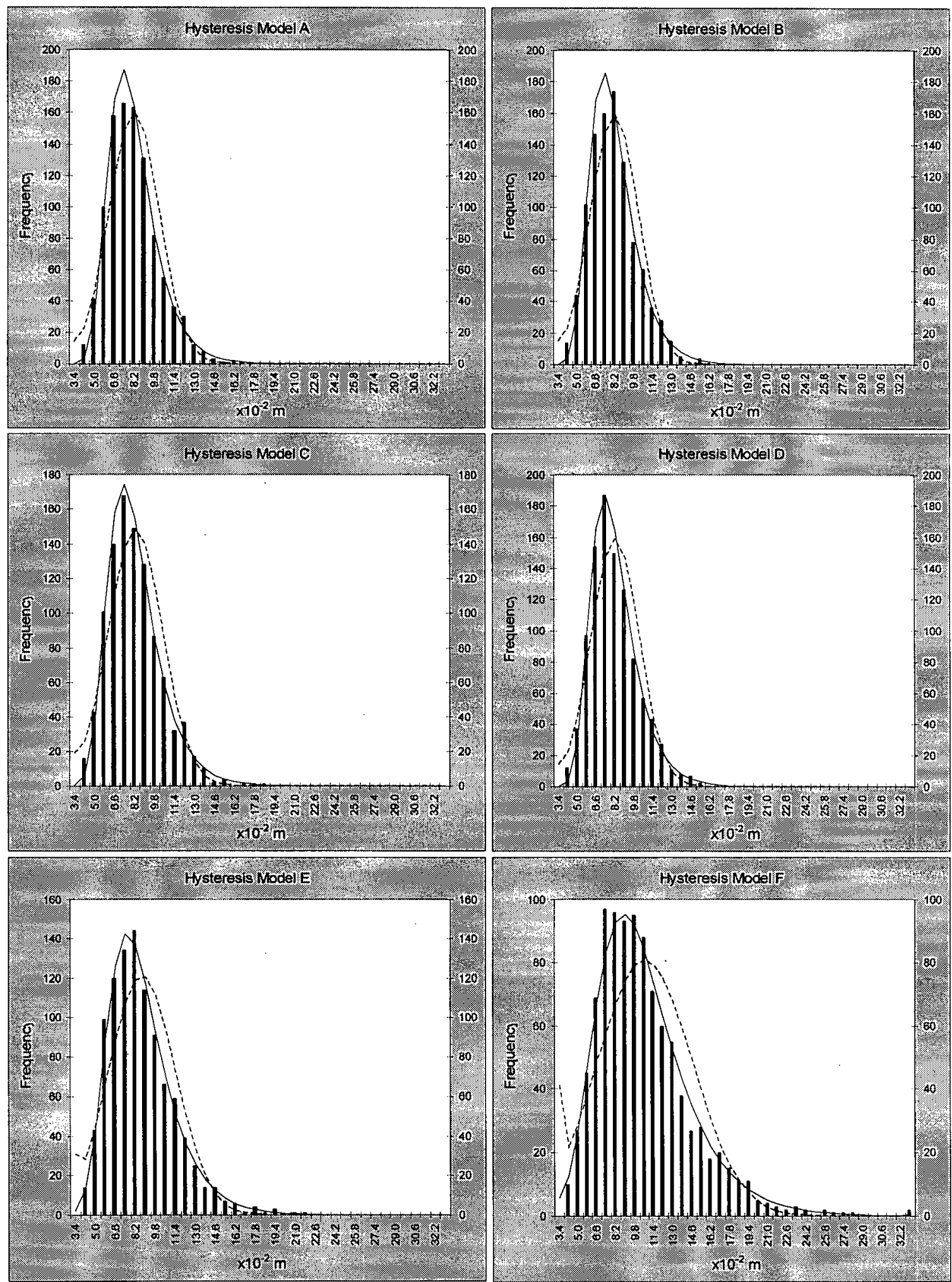

Figure 5.26: Displacement Response Histograms, $T=1.0 \mathrm{sec}$, Damping $=2 \%$ 

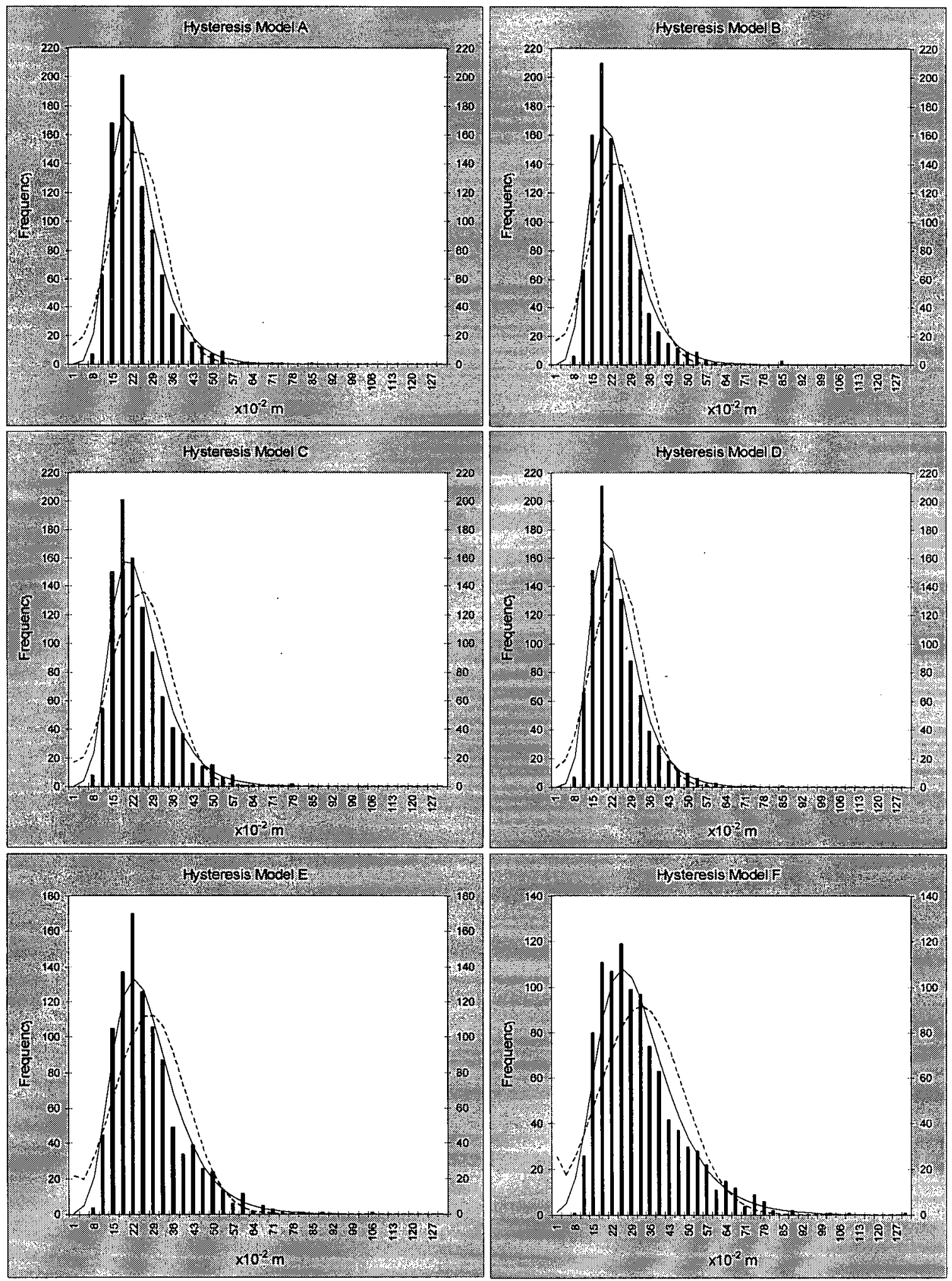

Figure 5.27: Displacement Response Histograms, $T=3.0 \mathrm{sec}$, Damping $=2 \%$ 

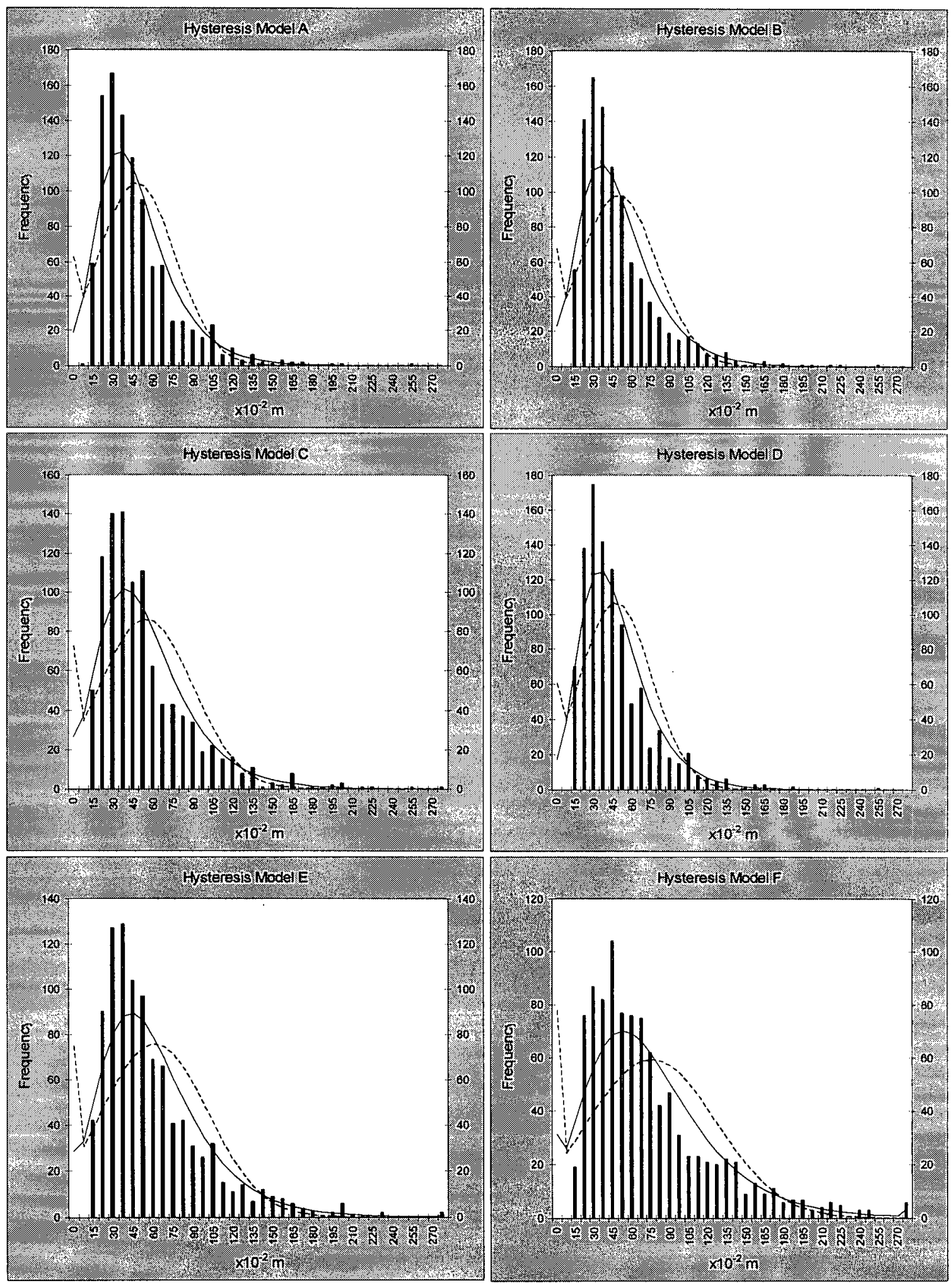

Figure 5.28: Displacement Response Histograms, $T=8.0 \mathrm{sec}$, Damping $=2 \%$ 
- From Figure 5.21 - Figure 5.28 it is apparent that the Gumbel distribution is again a better description of the peak deformation data in each case than the Normal distribution. Therefore, using the Gumbel distribution as a basis for comparison, Figure 5.29 summarizes the individual distributions at each period, including the distribution of peak elastic deformations, to illustrate the effect of the different hysteresis models and their agreement with the elastic distribution across the range of natural periods.

Beginning with the question of the effect of hysteresis model properties on peak displacement response, a number of observations can be made from Figure 5.29 and Table 5.10, which lists the dispersion parameter and mode describing the Gumbel distribution for each set of results. Note that the dispersion parameter is analogous to the variance of a Normal distribution while the mode is the location of the peak in the distribution. Comparing only the hysteresis models with each other, the modes of each distribution are located at essentially the same peak displacement for a given natural period. There was a tendency for the modes of the lower yield strength models, Hysteresis $\mathrm{E}$ and Hysteresis $\mathrm{F}$, to occur at slightly higher peak displacements, especially at the longer natural periods with their larger peak displacements, but this tendency was relatively small. At natural periods of 0.1 seconds and 0.2 seconds there was negligible difference between the hysteresis models because the peak displacements did not reach yield for any of the models. With increasing peak displacements at the longer natural periods, the dispersion of the distributions for the lower yield strength models and Hysteresis $\mathrm{C}$, with degradation parameters quadrupled, was increased with respect to the other models, particularly for Hysteresis $\mathrm{F}$ with $25 \%$ yield strength. This tendency was not particularly significant for Hysteresis $\mathrm{C}$ but there were definite differences from Hysteresis A and B, which had smaller degradation parameters. Only at the large peak displacements of $\mathrm{T}=3.0$ seconds and $\mathrm{T}=8.0$ seconds did the distribution of Hysteresis $\mathrm{B}$, with degradation parameters doubled, become noticeably different from Hysteresis A. At all natural periods, the distributions of Hysteresis A and D, which represented $100 \%$ yield strength with and without degradation parameters, were negligibly different. 

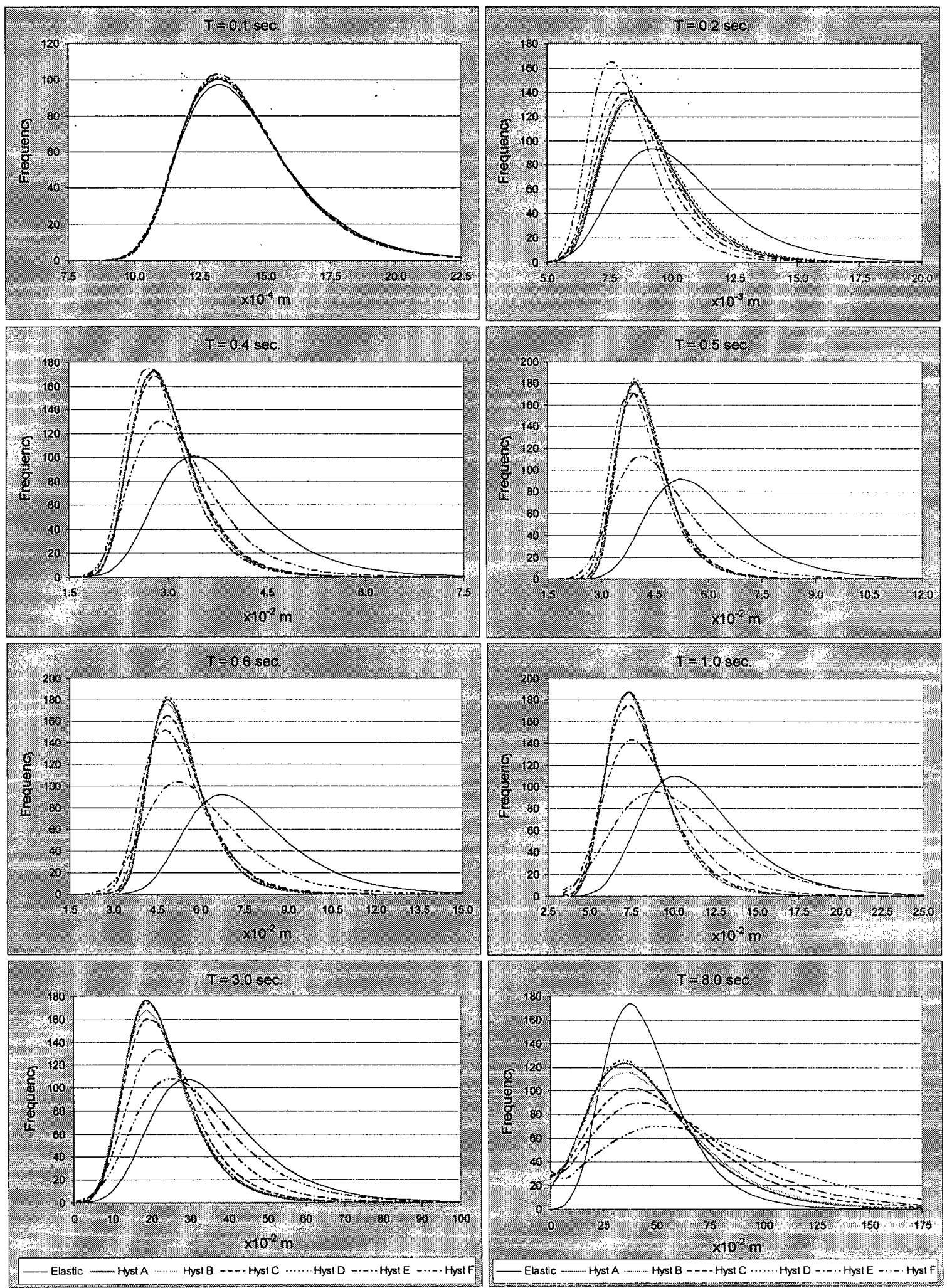

Figure 5.29: Hysteresis Model Comparison of Peak Deformation Distributions 
Table 5.10: Gumbel Distribution Parameters for Peak Deformation

\begin{tabular}{|c|c|c|c|c|c|c|c|}
\hline +8. & Statistic & Hyst A & Hyst B & Hyst C & Hyst D & forst $\mathrm{E}$ & Hyst F \\
\hline \multirow{2}{*}{$0.1 \mathrm{sec}}$. & Dinnorion & 479 & 5501.9051 & 5460.1337 & 5531.1127 & 5603.9804 & 5623.0013 \\
\hline & & 0.0 & 0 & 13 & 0.0 & 0.0013 & 0.00 \\
\hline \multirow{2}{*}{$0.2 \mathrm{sec}$} & & 728 & 737.2292 & 760.2819 & 208.0143 & 812.1206 & 905.2658 \\
\hline & Mo & 0.0 & 0.0080 & 0.0079 & 0.0081 & 0.0077 & 0.0073 \\
\hline \multirow{2}{*}{$0.4 \mathrm{sec}$} & Dis! & 238.0 & 235.7321 & 230.5929 & 237.6564 & 242.4594 & 177.7142 \\
\hline & & 0.0 & 0 & 0.0268 & 0 & 0.0260 & 00 \\
\hline \multirow{2}{*}{$0.5 \mathrm{sec}$} & & 166 & & 155.7097 & 169.1176 & 157.5203 & 102.5202 \\
\hline & Mode & 0.0379 & 0.0379 & $\sqrt{0.0}$ & 0.0381 & 0.0367 & 0.0398 \\
\hline \multirow{2}{*}{$0.6 \mathrm{sec}$} & Dis & 123 & 120.6 & 112.9021 & 125.3194 & 103.6185 & 70.3339 \\
\hline & & 0.0466 & 0.0464 & 0.0464 & 0.0467 & 0.0453 & 0.0502 \\
\hline \multirow{2}{*}{ (7) $1.0 \mathrm{sec}$} & & & 64.1057 & 599523 & 613180 & 48.7978 & 32.4550 \\
\hline & Mode & 0.0687 & 0.0685 & 0.0689 & 0.0690 & 0.0713 & 0.0847 \\
\hline \multirow{2}{*}{$3.0 \mathrm{sec}$. } & Disp & 13.8 & 13 & 12.5436 & 13.6 & 10.3990 & 8.4201 \\
\hline & se & 0. & 0.1702 & 0.1766 & 0.1721 & 0.1987 & 0.2334 \\
\hline $8: 0 \mathrm{sec}$ & Mo & $\frac{4.4926}{0.3082}$ & $\frac{4.2133}{0.3160^{\circ}}$ & 0.3 & $\frac{3 x-5802}{0.3069}$ & $\frac{3.2489}{0.3903}$ & $\frac{2.5440}{0.4883}$ \\
\hline
\end{tabular}

To enable a quantitative comparison of the effect of each hysteresis model on the distribution of peak deformation response at each natural period, the fitted Gumbel distributions of Figure 5.29 were used to evaluate the reliability index $\beta$ of each hysteresis model associated with structural drift limits of; $0.5 \%, 1 \%, 2 \%$ and $4 \%$, respectively. This type of analysis is an example of the intended purpose of PSResponse, which is to enable the evaluation of structural reliability under earthquake loading.

The displacement limits corresponding to the chosen drift limits were determined from a structural height that was calculated by assuming that the experimental lateral stiffness data of Figure 5.19 was taken from a rigid beam portal frame structure with 0.2 $\mathrm{m} \times 0.2 \mathrm{~m}$ timber columns. The resulting frame height of $5.34 \mathrm{~m}$ gives displacement 
limits of; $0.027 \mathrm{~m}, 0.053 \mathrm{~m}, 0.107 \mathrm{~m}$ and $0.214 \mathrm{~m}$, respectively, for the chosen drift limits. The performance function for this reliability analysis may then be expressed as:

$$
G=X-x
$$

where $X$ is the displacement limit and $x$ is the peak displacement random variable. The probability of failure and the associated reliability index may then be simply determined as:

$$
\begin{aligned}
& p_{f}=P(G \leq 0)=P(x \geq X) \\
& \beta=-\Phi^{-1}\left(p_{f}\right)
\end{aligned}
$$

Evaluation of the exceedence probability in Eq. [2] was done using the cumulative distribution function of the Gumbel distribution given by:

$$
F_{X}(x)=\exp [-\exp (-a(x-b))]
$$

where $a$ is the dispersion of the distribution and $b$ is the mode of the distribution. Table 5.11 lists the calculated probabilities of exceeding each displacement (drift) limit for each

\begin{tabular}{|c|c|c|c|c|c|c|c|c|}
\hline $\mathrm{T}$ & Limit & Elastic & Hyst A & Dyst B & Hyst C & Hyst D & Hyst E & Hyst $\mathrm{C}$ \\
\hline \multirow{4}{*}{$0.1 \mathrm{sec}$} & $0.027 \mathrm{~m}$ & 0 & 0 & 0 & 0 & 0 & 0 & 0 \\
\hline & $0.053 \mathrm{~m}$ & 0 & 0 & 0 & 0 & 0 & 0 & 0 \\
\hline & $0.107 \mathrm{~m}$ & 0 & 0 & 0 & 0 & 0 & 0 & 0 \\
\hline & $0.214 \mathrm{~m}$ & 0 & 0 & 0 & 0 & 0 & 0 & 0 \\
\hline & $0.027 \mathrm{~m}$ & 0.0001 & $20^{\circ}$ & 0 & 0 & 8 & 0 & 0 \\
\hline & $0.053 \mathrm{~m}$ & $3=0$ & 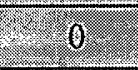 & $2.0 \%$ & 0 & $\begin{array}{l}-0 \\
-0\end{array}$ & 0 & $2.0 \%$ \\
\hline & $0.107 \mathrm{~m}$ & $0^{\circ}$ & $0^{0}$ & $\sqrt{7}+0^{2}=$ & 0 & 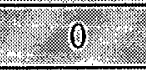 & 0 & -0.0 \\
\hline & $0.214 \mathrm{~m}$ & $=0$ & 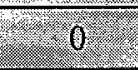 & $\overline{0}$ & 0 & $2=0$ & 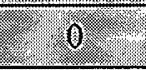 & 0 \\
\hline
\end{tabular}
hysteresis model at each natural period and Table 5.12 shows the reliability indices associated with the calculated exceedence probabilities.

Table 5.11: Displacement (Drift) Limit Exceedence Probabilities 


\begin{tabular}{|c|c|c|c|c|c|c|c|c|}
\hline 102 & I Iminit & WTlastic & Hyst 4 & Pryst B & Hyst d & Tryst D & Hiryst & Hyst $\mathrm{T}$ \\
\hline \multirow{4}{*}{$0.4 \mathrm{sec}$} & $0.027 \mathrm{~m}$ & 0.8960 & 0.6035 & 0.6015 & 0.6167 & 0.6111 & 0.5474 & 0.6902 \\
\hline & $0.053 \mathrm{~m}$ & 0.0604 & 0.0019 & 0.0020 & 0.0024 & 0.0020 & 0.0014 & 0.0115 \\
\hline & $0.107 \mathrm{~m}$ & 0 & 0 & 0 & 0 & 0 & 0 & 0 \\
\hline & $0.214 \mathrm{~m}$ & 0 & 0 & 0 & 0 & 0 & 0 & 0 \\
\hline \multirow{4}{*}{ 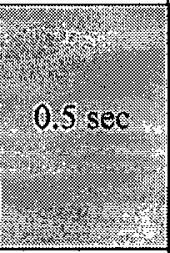 } & $0.027 \mathrm{~m}$ & $=0.9994 \%$ & 0.9979 & 009973 & -0.9954 & 0.9985 & 0.9898 & 099761 \\
\hline & $0.053 \mathrm{~m}$ & 0.5697 & 80.0787 & 0.0803 & 0.0895 & 0.0770 & 0.0735 & 0.2287 \\
\hline & $0.107 \mathrm{~m}$ & $\sqrt{100094}$ & Pry & 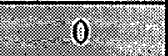 & $\sqrt{7 \times 10}$ & $\sqrt{7}, 0^{1}$ & $\sqrt{2}=0$ & $(0.0010$ \\
\hline & $0214 \mathrm{~m}$ & 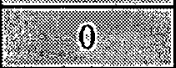 & 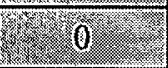 & $\sqrt{6} 0$, & $\sqrt{2 \% \sqrt{2}}$ & $8=0$ & 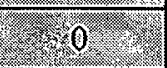 & $\sqrt{2}: \sqrt{0}$ \\
\hline \multirow{4}{*}{$0.6 \mathrm{sec}$} & $0.027 \mathrm{~m}$ & 1 & 1 & 1 & 0.9999 & 1 & 0.9987 & 0.9940 \\
\hline & $0.053 \mathrm{~m}$ & 0.8846 & 0.3634 & 0.3639 & 0.3786 & 0.3642 & 0.3634 & 0.5601 \\
\hline & $0.107 \mathrm{~m}$ & 0.0714 & 0.0006 & 0.0007 & 0.0011 & 0.0005 & 0.0017 & 0.0182 \\
\hline & $0.214 \mathrm{~m}$ & 0.0001 & 0 & 0 & 0 & 0 & 0 & 0 \\
\hline \multirow{4}{*}{ 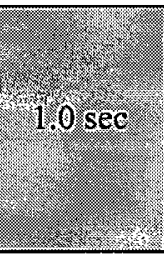 } & $0.027 \%$ & & & $\sqrt{27 \times 19}$ & 2 & $\sqrt[7]{7}$ & 0.9998 & 0.9985 \\
\hline & $20.053 \mathrm{~m}$ & 1009952 & 0.9361 & 0.9326 & 0.9248 & 20.9393 & 0.9 .9134 & 0.9393 \\
\hline & $0.107 \mathrm{~m}$ & 0.5046 & 0.0805 & 0.0812 & 010966 & 0.0832 & 0.1609 & 0.3846 \\
\hline & $0.214 \mathrm{~m}$ & (20.0125 & 30.0001 & 0.0001 & 0.0002 & 0.0001 & 0.0009 & 0.0150 \\
\hline \multirow{4}{*}{$3.0 \mathrm{sec}$} & $0.027 \mathrm{~m}$ & 0.9996 & 0.9993 & 0.9985 & 0.9985 & 0.9993 & 0.9974 & 0.9966 \\
\hline & $0.053 \mathrm{~m}$ & 0.9982 & 0.9938 & 0.9904 & 0.9911 & 0.9937 & 0.9894 & 0.9896 \\
\hline & $0.107 \mathrm{~m}$ & 0.9822 & 0.9098 & 0.8987 & 0.9089 & 0.9120 & 0.9254 & 0.9449 \\
\hline & $0.214 \mathrm{~m}$ & 0.8064 & 0.4219 & 0.4307 & 0.4652 & 0.4318 & 0.5739 & 0.6920 \\
\hline \multirow{4}{*}{$8.0 .5 e c$} & $0.0027 \mathrm{n}$ & 0.9991 & 0.9709 & $.0,9659$ & 809627 & 0.9728 & 0.9614 & 0,9606 \\
\hline & $0.053 \mathrm{~m}$ & 0.9974 & $0.9570^{\circ}$ & $0.9516^{\circ}$ & 0.9496 & $0.9592 \%$ & 0.9498 & 0.9515 \\
\hline & $0.107 \mathrm{~m}$ & 09854 & 0.9153 & 809104 & 0.9134 & 0.9178 & $\sqrt{0.9187}$ & 0.9285 \\
\hline & $0.214 \mathrm{~m}$ & 0.8826 & 0.7828 & 0.7850 & 80.8075 & 0.7836 & 0.8302 & .0 .865 \\
\hline
\end{tabular}

Table 5.12: Displacement (Drift) Limit Reliability Indices

\begin{tabular}{|c|c|c|c|c|c|c|c|c|}
\hline$\sqrt{T} / 2 \%$ & Timit & Elastic & myst A & Hyst B & Myst C. & Hyst B & fryst $\mathrm{E}$ & Hyst F \\
\hline \multirow{4}{*}{$0.1 \mathrm{sec}$} & $0.027 \mathrm{~m}$ & 8 & 8 & 8 & 8 & 8 & 8 & 8 \\
\hline & $0.053 \mathrm{~m}$ & 8 & 8 & 8 & 8 & 8 & 8 & 8 \\
\hline & $0.107 \mathrm{~m}$ & 8 & 8 & 8 & 8 & 8 & 8 & 8 \\
\hline & $0.214 \mathrm{~m}$ & 8 & 8 & 8 & 8 & 8 & 8 & 8 \\
\hline \multirow{4}{*}{ ( } & $0.027 \mathrm{~m}$ & 3,7086 & 4.7531 & 47946 & 4.8966 & 4.6621 & 51166 & 55062 \\
\hline & $0.053 \mathrm{~m}$ & 162656 & 1771611 & $\frac{7.7706}{7.76}$ & 8 & 7.759 & 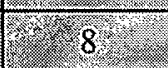 & 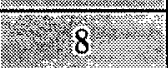 \\
\hline & $0.107 \mathrm{~m}$ & 8 & 8 & 8 & & $\sqrt{9}$ & $=\sqrt[7]{ } \cdot \sqrt{8}$ & 8 \\
\hline & $0.214 \mathrm{~m}$ & $\sqrt[3]{8}$ & & & 8 & & & $\sqrt{1.8}$ \\
\hline
\end{tabular}




\begin{tabular}{|c|c|c|c|c|c|c|c|c|}
\hline T & Iinit & Elastic & Tyst 4 & Hyst B & Hyst C & Hyst $\mathbf{D}$ & Hyst E & Hyst F \\
\hline \multirow{4}{*}{$0.4 \mathrm{sec}$} & $0.027 \mathrm{~m}$ & -1.2590 & -0.2625 & -0.2573 & -0.2969 & -0.2821 & -0.1192 & -0.4964 \\
\hline & 0.05 & 1.5515 & 2.8951 & 2.8777 & 2.8222 & 2.8853 & 2.9784 & 2.2743 \\
\hline & $0.107 \mathrm{~m}$ & 3.9710 & 5.7328 & 5.7020 & 5.6243 & 5.7238 & 5.8180 & 4.8026 \\
\hline & 0.21 & 6.6620 & 8 & 8 & 8 & 8 & 8 & 7.7571 \\
\hline \multirow{4}{*}{$0.5 \mathrm{sec}$} & $0.027 \mathrm{~m}$ & -3.2187 & -2.8584 & $2-2.7823$ & -2.6016 & -2.9694 & -2.3180 & -1.9785 \\
\hline & $0.053 \mathrm{~m}$ & -0.1756 & 1.4139 & 1.4031 & 1.3439 & 1.4255 & 1.4505 & 0.7433 \\
\hline & $0.107 \mathrm{~m}$ & 2.3511 & 4.2555 & 42227 & 4.0973 & 4.2970 & 4.1672 & 3.0835 \\
\hline & $0.214 \mathrm{~m}$ & 4.7051 & 7.2567 & 672032 & 70082 & 7.3254 & 7.0775 & 5.5136 \\
\hline \multirow{4}{*}{$0.6 \mathrm{sec}$} & $0.027 \mathrm{~m}$ & -4.1384 & -4.1923 & -4.0139 & -3.6553 & -4.3232 & -3.0221 & -2.5111 \\
\hline & $0.053 \mathrm{~m}$ & -1.1985 & 0.3493 & 0.3481 & 0.3091 & 0.3474 & 0.3494 & -0.1513 \\
\hline & $0.107 \mathrm{~m}$ & 1.4652 & 3.2512 & 3.2074 & 3.0701 & 3.2790 & 2.9334 & 2.0915 \\
\hline & $0.214 \mathrm{~m}$ & 3.7377 & 5.9899 & 5.9157 & 5.6982 & 6.0381 & 5.4469 & 4.2668 \\
\hline \multirow{4}{*}{$\begin{array}{l}0 \\
160 \mathrm{sec}\end{array}$} & 0.02 & -4.8320 & -4.9423 & -4.8473 & -4.4371 & -4.9719 & 3.5885 & $=2.9712$ \\
\hline & $0.053 \mathrm{~m}$ & -2.5923 & -15230 & -1.4956 & -1.4380 & -1.5489 & -1.3621 & -1.5486 \\
\hline & $0.107 \mathrm{~m}$ & -0.0115 & 1.4019 & 1.3974 & 1.3012 & 1.3836 & 0.9906 & 0.2934 \\
\hline & $0.214 \mathrm{~m}$ & 2.2412 & 3.7652 & 3.7489 & 3.5886 & 3.7480 & 3.1064 & 2.1713 \\
\hline \multirow{4}{*}{$3.0 \mathrm{sec}$} & $0.027 \mathrm{~m}$ & -3.3665 & -3.1968 & -2.9769 & -2.9777 & -3.1846 & -2.7980 & -2.7072 \\
\hline & $0.053 \mathrm{~m}$ & -2.9149 & -2.4980 & -2.3416 & -2.3677 & -2.4968 & -2.3058 & -2.3124 \\
\hline & $0.10^{7}$ & -2.1006 & -1.3396 & -1.2741 & -1.3339 & -1.3529 & -1.4422 & -1.5975 \\
\hline & $0.214 \mathrm{~m}$ & -0.8647 & 0.1971 & 0.1745 & 0.0873 & 0.1718 & -0.1862 & -0.5014 \\
\hline \multirow{4}{*}{$80 \mathrm{sec}$} & $0.027 \mathrm{~m}$ & -3.126 & 718941 & -1.8240 & -1.7826 & -1.9236 & -1.7674 & -17574 \\
\hline & $0.053 \mathrm{~m}$ & -2.7965 & 217171 & -1.6607 & 1.6406 & -1.7419 & -1.6428 & -1.6597 \\
\hline & $0.107 \mathrm{~m}$ & $-2,1814$ & -1.3743 & -1.3432 & -1.3621 & -1.3905 & -1.3966 & -1.4646 \\
\hline & $0.214 \mathrm{~m}$ & 41.1883 & -0.7815 & -0.7890 & -0.8688 & -0.7844 & -0.9549 & -1.1073 \\
\hline
\end{tabular}

Examining the reliability indices of Table 5.12 reveals three key trends in the drift exceedence probabilities. Firstly, for each drift limit at each natural period the elastic response gives a conservative estimate of drift reliability as evidenced by the smaller $\beta$ value compared to each of the six hysteresis models. Secondly, the $\beta$ values for the hysteresis models vary significantly at a given natural period and drift limit. Thirdly, the range of variability in the $\beta$ values for the hysteresis models is dependent on the natural period and chosen drift limit. For example, the reliability index for $\mathrm{T}=1.0$ seconds and 
$1 \%$ drift $(0.053 \mathrm{~m})$ ranged between -1.3621 and -1.5489 , while for $2 \% \operatorname{drift}(0.107 \mathrm{~m})$ at the same natural period, the reliability index ranged between 1.4019 and 0.2934 .

The latter two key observations indicate that the characteristics of a hysteresis model have a significant effect on the calculated seismic reliability of a structure, with the effect being more or less pronounced depending on the capacity limit that is used to assess seismic reliability. Taken together, these observations show that the hysteretic behaviour of a structure needs to be accurately modeled, particularly in shorter natural period structures, to provide an accurate probabilistic description of response and hence a good estimate of seismic structural reliability. The natural period dependence of the relative importance of an accurate hysteresis model can be seen in the basic equation of motion for a structure, which can be written as follows:

$$
\ddot{x}+2 \xi \omega_{n} \dot{x}+\frac{F(x)}{m}=-\ddot{a}_{g}
$$

From that form of the equation of motion it is clear that the relative importance of the restoring force $F(x)$ is reduced with increasing mass for a given structural stiffness. Therefore, the shape of the hysteresis loop for longer period structures is relatively less important when determining the probabilistic response of the structure.

Turning to the question of the appropriateness of the equal displacement observation in structural dynamics, it is clear from Figure 5.29 that the distributions of elastic peak displacement differ from the inelastic distributions for all natural periods exhibiting a significant response. In general, the modes of the inelastic distributions are located at a lower peak displacement than the modes of the corresponding elastic distributions, which were close to the spectral displacement for the El Centro ground motion at each period (see Fig. 5.18). This observed trend suggests that the elastic response of a structure tends to give a conservative estimate of peak inelastic displacement, which was noted previously in the analysis of drift reliability. To enable evaluation of this observed conservativeness and identify how it changes with natural 
period and relative yield strength, the percentage difference between the elastic and inelastic peak displacement for each record in the sequence of 1000 generated ground motions was determined at each natural period using three hysteresis models; Hysteresis D, Hysteresis $\mathrm{E}$ and Hysteresis F. These represent non-degrading hysteretic behaviour . with $100 \%$ yield strength, $50 \%$ yield strength and $25 \%$ yield strength, respectively. Determining the probabilistic description of the percentage difference between peak elastic and peak inelastic displacement then allows for a reliability analysis of the conservativeness of the equal displacement principle.

Table 5.13 lists the basic statistics for the distribution of each set of percentage difference results and Figure 5.30 shows the histogram plots of the actual frequency distribution of the results for two natural periods. These plots are once again overlaid with the associated Gumbel distribution, given as a solid line, and Normal distribution, given as a dashed line, to illustrate the fit of each type of distribution to the data.

Table 5.13: Percentage Difference Statistics for Inelastic vs. Elastic Peak Deformation

\begin{tabular}{|c|c|c|c|c|}
\hline$P$ & Statistic & Hyst D & Hyst $\mathrm{E}$ & Hyst F \\
\hline \multirow{4}{*}{$0.1 \mathrm{sec}$} & Min. & -25.64 & -25.23 & -28.60 \\
\hline & Max. & 20.45 & 33.41 & 34.40 \\
\hline & Avg. & -0.46 & -0.42 & -0.33 \\
\hline & Stdev. & 4.91 & 6.34 & 7.84 \\
\hline & . Min. & -33.56 & $\begin{array}{r}44.89 \\
\end{array}$ & -55.61 \\
\hline & Max & 16.75 & 22.40 & 31.25 \\
\hline & Avg. & -10.58 & -15.28 & -19.14 \\
\hline & (7) Stdev: & $\sqrt{7.40}$ & $\sqrt{10.24}$ & 13.50 \\
\hline \multirow{4}{*}{$0.4 \mathrm{sec}$. } & Min. & -52.25 & -58.31 & -60.70 \\
\hline & Max. & 31.69 & 53.06 & 123.04 \\
\hline & Avg. & -19.17 & -20.45 & -12.08 \\
\hline & Stdev. & 13.35 & 17.88 & 26.25 \\
\hline \multirow{4}{*}{ (2) 0.5 sec. } & Min. & -59.69 & -67.54 & 2.70 .91 \\
\hline & Max & 23.38 & 66.37 & 114.47 \\
\hline & 2.74. & 0.25 .62 & -27.02 & 10.17 .11 \\
\hline & Stdev. & 214.60 & 18.71 & 28.47 \\
\hline
\end{tabular}




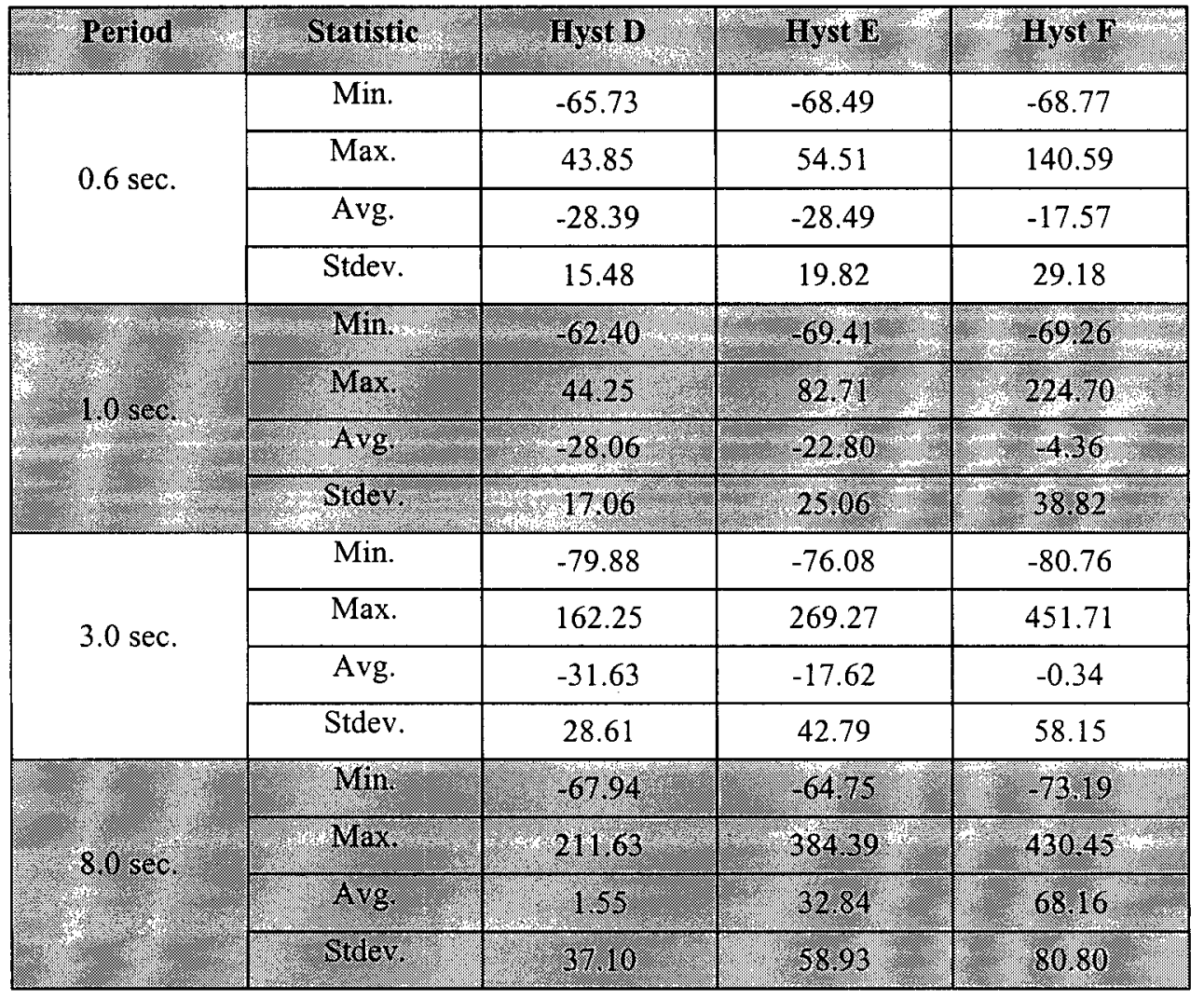

From Figure 5.30, the Gumbel distribution once again appears to be a better description of the percentage difference data than the Normal distribution, therefore, using the Gumbel distribution as a basis for comparison, Figure 5.31 summarizes the individual distributions of each set of percentage difference results for each hysteresis model at each natural period. Also, Table 5.14 lists the dispersion parameter and mode describing the Gumbel distribution for each set of results.

Table 5.14: Gumbel Distribution Parameters for Percentage Difference Results

\begin{tabular}{|c|c|c|c|c|}
\hline Period & Statistic & Hyst D & Hyst E & Hyst F \\
\hline \multirow{2}{*}{$0.1 \mathrm{sec}}$. & Dispersion & 0.26 & 0.20 & 0.16 \\
\cline { 2 - 5 } & Mode & -2.67 & -3.27 & -3.86 \\
\hline \multirow{3}{*}{$0.2 \mathrm{sec}}$. & Dispersion & 0.17 & 0.13 & 0.09 \\
\cline { 2 - 5 } & Mode & -13.91 & -19.89 & -25.22 \\
\hline \multirow{2}{*}{$0.4 \mathrm{sec}}$. & Dispersion & 0.10 & 0.07 & 0.05 \\
\cline { 2 - 5 } & Mode & -25.18 & -28.49 & -23.89 \\
\hline
\end{tabular}




\begin{tabular}{|c|c|c|c|c|}
\hline Period & Statistic & Hyst D & Hyst E & Hyst F \\
\hline \multirow{2}{*}{$0.5 \mathrm{sec}}$. & Dispersion & 0.09 & 0.07 & 0.05 \\
\cline { 2 - 5 } & Mode & -32.19 & -35.45 & -29.92 \\
\hline \multirow{2}{*}{$0.6 \mathrm{sec}}$. & Dispersion & 0.08 & 0.06 & 0.04 \\
\cline { 2 - 5 } & Mode & -35.36 & -37.41 & -30.70 \\
\hline \multirow{2}{*}{$1.0 \mathrm{sec}}$. & Dispersion & 0.08 & 0.05 & 0.03 \\
\cline { 2 - 5 } & Mode & -35.74 & -34.08 & -21.83 \\
\hline \multirow{2}{*}{$3.0 \mathrm{sec}$} & Dispersion & 0.04 & 0.03 & 0.02 \\
\hline \multirow{2}{*}{$8.0 \mathrm{sec}}$. & Mode & -44.50 & -36.88 & -26.51 \\
\cline { 2 - 5 } & Dispersion & 0.03 & 0.02 & 0.02 \\
\cline { 2 - 5 } & Mode & -15.15 & 6.32 & 31.79 \\
\hline
\end{tabular}

Using the fitted Gumbel distributions of Figure 5.31, the probability that the percentage difference between peak inelastic and peak elastic displacement exceeded $0 \%$ was evaluated for each hysteresis model (i.e. yield level) at each natural period. This then allows for a reliability estimate of the conservativeness of the equal displacement principle. Note that a negative percentage difference indicates that the peak elastic displacement exceeded the peak inelastic displacement, thereby giving a conservative estimate of peak displacement. Table 5.15 lists the calculated exceedence probabilities for each hysteresis model at each natural period.

Table 5.15: Zero Percent Difference Exceedence Probabilities

\begin{tabular}{|c|c|c|c|c|c|c|c|c|}
\hline & $\mathrm{T}=\mathbf{0 . 1} \mathrm{s}$ & $\mathrm{T}=0.2 \mathrm{~s}$ & $\mathrm{~T}=0.4 \mathrm{~s}$ & $\mathrm{~T}=\mathbf{0 . 5 \mathrm { s }}$ & $\mathrm{T}=\mathbf{0 . 6 \mathrm { s }}$ & $\mathrm{T}=\mathbf{1 . 0 \mathrm { s }}$ & $\mathrm{T}=\mathbf{3 . 0 \mathrm { s }}$ & $\mathrm{T}=\mathbf{8 . 0 \mathrm { s }}$ \\
\hline Hyst D & 0.3924 & 0.0858 & 0.0853 & 0.0575 & 0.0520 & 0.0659 & 0.1272 & 0.4469 \\
\hline Hyst E & 0.4029 & 0.0795 & 0.1215 & 0.0843 & 0.0850 & 0.1603 & 0.2818 & 0.6826 \\
\hline Hyst F & 0.4127 & 0.0871 & 0.2674 & 0.2287 & 0.2285 & 0.3850 & 0.4272 & 0.8092 \\
\hline
\end{tabular}




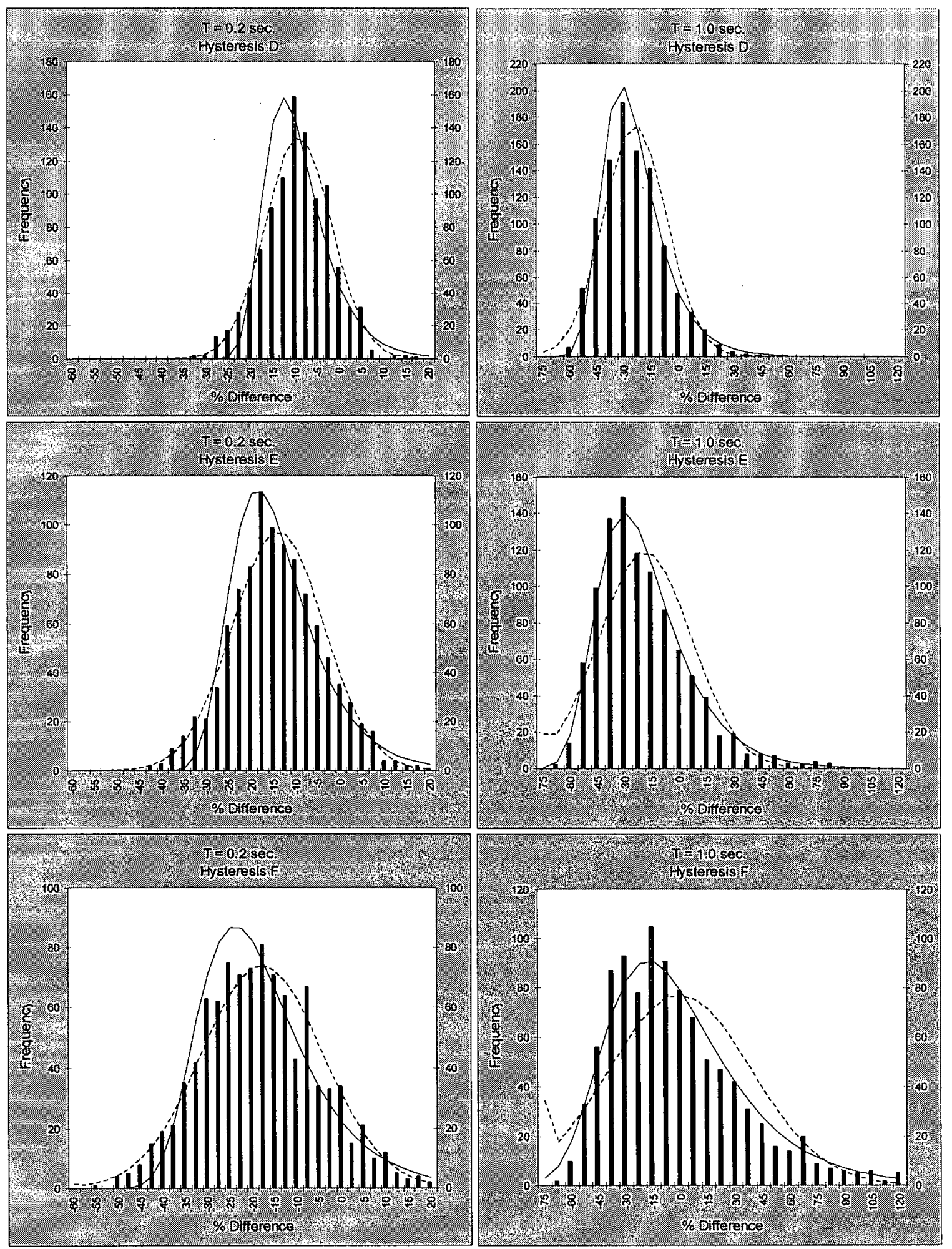

Figure 5.30: Percentage Difference Histograms for Inelastic vs. Elastic Peak Deformation 

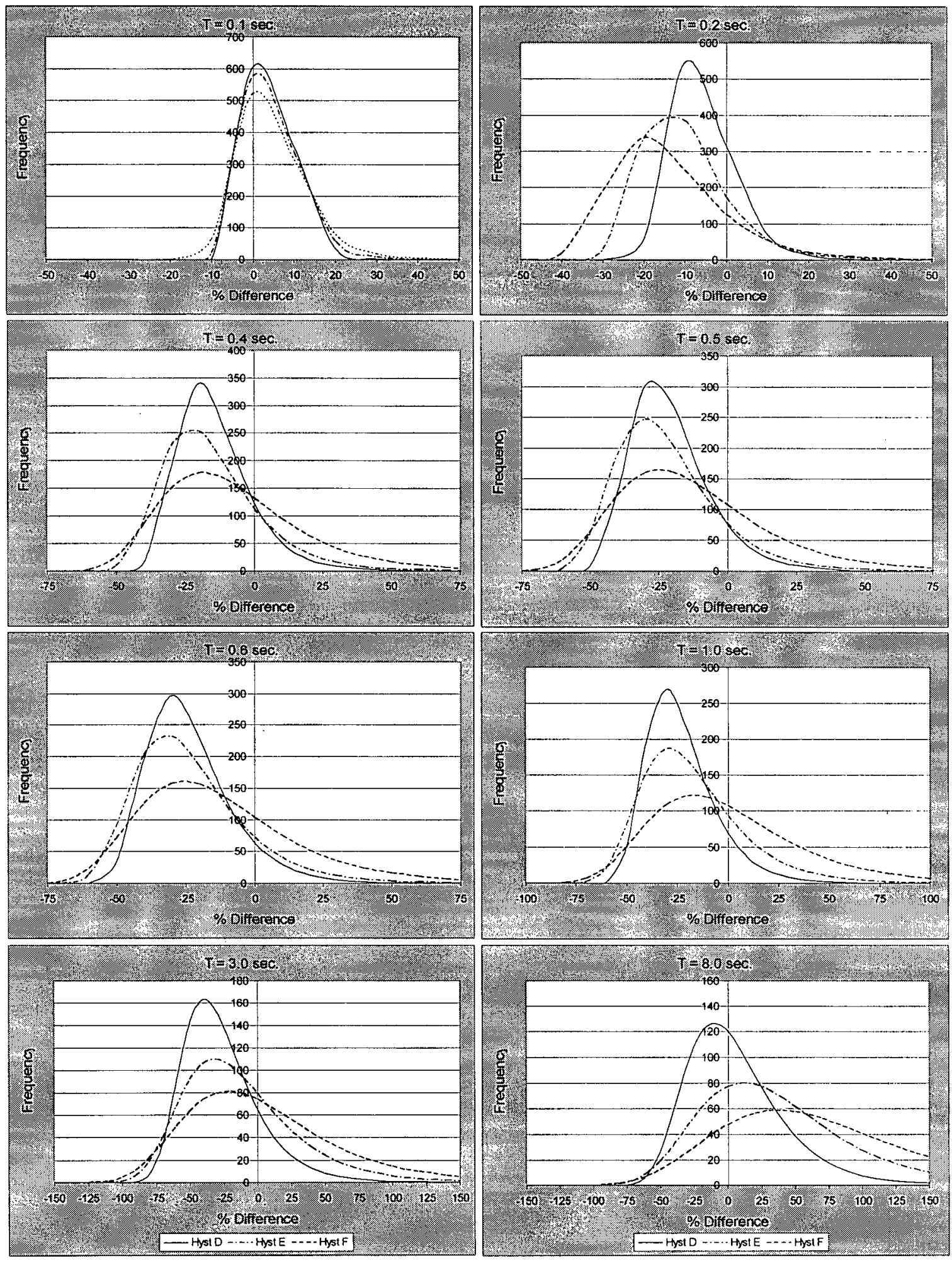

Figure 5.31: Percentage Difference Distributions for Inelastic vs. Elastic Peak Deformation 
Examining the Gumbel distributions of Figure 5.31 and the associated reliability indices of Table 5.16 it is clear that the peak elastic displacement of a structure generally gave a conservative estimate of peak inelastic displacement (elastic $\geq$ inelastic). This trend was consistent except for the longer natural period structures $(T=3.0 \mathrm{sec}, \mathrm{T}=8.0$ sec) with reduced yield strength (Hyst E, Hyst F) where very large peak displacements, which exceeded peak ground displacement by a factor of 2 to 3 , were commonly observed (see Fig. 5.18 and Fig. 5.29). Also, for a given natural period the probability that peak elastic displacement gave a conservative estimate of peak inelastic displacement (elastic $\geq$ inelastic) was reduced as yield strength decreased. This is clearly evident from the increasing dispersion in the percentage difference distributions as yield strength decreased from $100 \%$ to $50 \%$ to $25 \%$ in Hysteresis D, Hysteresis $\mathrm{E}$ and Hysteresis F, respectively. The degree of dispersion consistently increased with increasing peak displacements as natural period increased.

While it is difficult to make definitive statements regarding the accuracy of the equal displacement principle owing to the wide spread in percentage difference results in Figure 5.31, the preceding observations indicate that the equal displacement principle is generally valid in the sense that elastic peak displacement provides a useful, conservative first approximation of inelastic peak displacement. 


\section{CHAPTER 6}

\section{SUMMARY, CONCLUSIONS AND RECOMMENDATIONS}

\subsection{SUMMARY}

The evaluation of seismic reliability of building structures is a complex and computationally expensive process since it requires, at the most fundamental level, the evaluation of the probabilistic dynamic response of a given structure to the stochastic dynamic action of an earthquake. Because of the difficulty of determining the response of a structure in a statistical sense, past estimates of the seismic reliability of existing structures, and typical structural systems, have been largely qualitative in nature. With the movement of many national building codes towards more performance-based design measures, a need was identified for a more quantitative method of evaluating structural reliability under seismic loading.

The overall objective of this study was then to develop a simple, useable software application for probabilistic analysis of the dynamic response of civil engineering structures to random ground motions. Knowing the probabilistic response of a structure, an accurate assessment of a specific reliability measure can be made from the probability of exceeding a chosen threshold. Using this approach, the aim was to provide a tool for engineers and researchers that could be used to evaluate the probable effect of a wide range of ground motion characteristics and structural model parameters, each with their own random nature, on the dynamic response of a structure.

To begin the process of developing the software application, a comprehensive review of random vibration methods and numerical procedures was carried out to identify a suitable method of analyzing the probabilistic seismic response of civil engineering structures. This review, which included all key research related to the mathematical 
modelling of probabilistic structural behaviour done over the past 50 years, assessed the limitations of each method with regard to the level of complexity in the structural model, degree of non-linearity in system restoring forces and nature of the random excitation process. To provide an accurate, robust and practical means of evaluating structural reliability under seismic loading, the chosen procedure had to allow for highly non-linear response behaviour, realistic stochastic structural models with multiple degrees of freedom, and realistic earthquake motions. These requirements tended to eliminate all the frequency-domain based analytical random vibration methods, with the exception of the Equivalent Linearization method, because they are too restrictive in their inherent assumptions to confidently apply their results to real structures experiencing realistic earthquakes.

The Markov-based methods assume a white noise excitation, which is a poor representation of a real earthquake spectrum, and most of them (Galerkin method, Finite Element method, and the Closure Technique) have the disadvantage of slow convergence or large computational requirements for highly non-linear or multiple-degree-offreedom systems. In the case of the Numerical Diffusion method, the applicability of Generalized Cell Mapping (GCM) to degrading hysteretic systems, which characterizes most structures, is not known.

The Perturbation method and the Functional Series Representation method are not confined to a white-noise assumption for the excitation and are even applicable to multiple-degree-of-freedom systems. However, these methods are limited to weakly non-linear systems and as such are not applicable to ductile, hysteretic structural systems.

The Equivalent Linearization method has been successfully applied to response and damage prediction of a variety of highly non-linear structural systems under seismic excitation. However, response results may tend to be underestimated using this method when the excitation spectral content is such that the power spectral density function vanishes rapidly as the frequency goes to zero, which is typical of earthquake excitation. In addition, the assumption of a Gaussian input excitation results in an assumed Gaussian 
response of the linearized non-linear system. This assumption is not correct for a nonlinear system, which is known to have a non-Gaussian response to a Gaussian input. The result of an assumed Gaussian response is that it may significantly misrepresent the frequency of high response levels to extreme loads, which contribute most to firstpassage and fatigue failures.

With these limitations in mind, the decision was made to forego the efficiency of the frequency-domain based methods in favour of a robust numerical time-history approach incorporating the Monte Carlo method. This approach, while more computationally demanding than the analytical procedures, allows the probabilistic response of a structure to be evaluated without regard to the degree of non-linearity in the restoring force, complexity of the structural system, nature of the variability in structural properties or nature of the random excitation process.

The decision to adopt a Monte Carlo, time-history approach for determining the probabilistic seismic response of a structure required that several types of component models be incorporated into the overall architecture of the software, which was named PSResponse. Models for generating and modulating artificial ground motion timehistories, structural models along with a means of simulating a non-linear, hysteretic restoring force, as well as an overall numerical time-stepping method to solve the differential equation of motion were linked together to form the computational foundation of the software. In addition, the overall framework of PSResponse required algorithms for Fourier analysis and power spectrum estimation, a frequency filtering algorithm to ensure that input ground accelerations were truly representative of a real earthquake, a long period random number generator to ensure a reliable source of random numbers essential for Monte Carlo analysis, and algorithms for solving the eigensystem representing the natural frequencies and mode shapes of a multiple-degree-of-freedom structure. In total, the computational engine of PSResponse consists of approximately 79 algorithms linked together in an object-oriented framework. 
The ground motion models that were incorporated into PSResponse are wellknown methods that reproduce the probabilistic characteristics of a specified frequency spectrum while the hysteresis model is a version of the well-known BWBN, or BoucWen, hysteresis model that was specifically modified for this software application. The BWBN model, which uses a smooth differential equation to represent the non-linear component of the restoring force in the equation of motion, was chosen over the use of piece-wise linear equations because it is able to reproduce a wide variety of hysteresis shapes, including pinching and degradation behaviour, without the use of numerous empirical rules governing the relationship of stiffness to displacement. It also allowed for the development of an algorithm within the software that identifies the parameters governing hysteretic behaviour from experimental data provided by the user. Thirteen separate parameters must be identified in the original BWBN model, although two of the parameters are typically set to unity, which is a computationally demanding process since system identification problems rapidly increase in difficulty as the number of parameters increases. To simplify the process, the BWBN model was modified to reduce the number of parameters controlling pinching behaviour from six to three using the assumption that overall structural hysteretic pinching begins at or very near zero restoring force in each loading cycle. With this modified pinching function, the role of each of the three new parameters is more easily understood than the relationship between the six parameters of the original pinching function. One parameter controls the overall rate of increase in pinching as damage cycles progress, a second parameter controls the rate of stiffness recovery throughout the loading phases of each cycle and a third parameter controls the rate of stiffness recovery during the increasing displacement portion of each loading phase. Incorporation of the modified BWBN hysteresis model into the numerical timestepping procedure required that a separate numerical solution algorithm be linked with the Newton-Raphson iteration scheme in the Newmark Method since the first-order, non-linear ordinary differential equation of the BWBN model has no exact solution.

Following development of the solution algorithms and numerical components that form the computational engine of PSResponse, a Windows user-interface was developed to provide easy access to the software and ensure the integrity of the input data prior to 
analysis. The user-interface is based on a wizard manager architecture that guides the user through a sequence of input and output dialog boxes that depends on the type of analysis selected. A wizard manager algorithm, which acts as the link between the dialog boxes and the computational framework, determines which of the 26 dialog boxes are required, passes information between dialog boxes, passes input data to the computational algorithms and stores both input data and output arrays.

The final phase in the development of PSResponse was verification of the accuracy of a calculated dynamic response against a reliable benchmark, which was chosen to be $\boldsymbol{S A P 2 0 0 0}$ Nonlinear 8.1.2 (Computers \& Structures Inc. 2003) because it is an accepted industry standard software package capable of performing highly sophisticated non-linear time-history analysis. As a further verification check, elastic and inelastic analysis results from each software application for both single-degree-offreedom and multiple-degree-of-freedom structures were compared with corresponding results published by Chopra (1995). This comparison showed good agreement between each set of calculated responses for each type of analysis; which was taken as confirmation that PSResponse results meet an acceptable standard of accuracy for the intended purpose of the software. This purpose is to provide fast, summary-level dynamic analysis results for determining the probabilistic response of linear and nonlinear systems under stochastic dynamic loading. To that end, it is worth noting that PSResponse was two orders of magnitude faster than SAP2000 in calculating the inelastic dynamic response of the five-storey shear structure subjected to the El Centro ground motion. Calculation time for PSResponse was less than one second on a $1.5 \mathrm{GHz}$

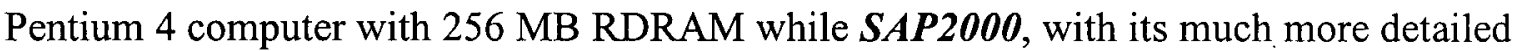
finite-element basis, took approximately 83 secọnds.

Finally, as a first application of the beta release of PSResponse, two case studies were done to demonstrate the capabilities of the program as a research and analysis tool. These case studies analyzed, from a probabilistic point of view, three general questions pertinent to structural dynamics and earthquake engineering; the relative effect of random structural properties on the dynamic response of a structure, the appropriateness of the 
well-known equal displacement observation and the effect of hysteresis model properties on displacement response.

The first case study analyzed the effect of random properties on the dynamic response of a structure by evaluating the probable peak base shear, peak base moment and peak fifth storey displacement of a five-storey elastic structure using a sequence of 1000 generated records and five levels of variability in the storey mass, storey stiffness, storey height and modal damping. As well, the probable accuracy of the Response Spectrum Analysis (RSA) procedure for determining the peak responses of a multipledegree-of-freedom system using the absolute sum (ABSSUM), square-root-of-sum-ofsquares (SRSS) and complete quadratic combination (CQC) modal combination rules was evaluated.

Results from the first case study showed that peak elastic response data is well described by the Gumbel distribution, which was then used as the basis for comparison in evaluating the effect of increasing structural parameter variability on the probability distribution of the chosen peak elastic responses. Using a number of statistical tests, the distribution of each peak response was compared at each level of structural variability with the distribution associated with a deterministic structure. From that analysis it was determined that the randomness of the generated ground motions accounts for the majority of the observed range in a given peak response while structural randomness had a relatively minor effect. This then suggests that careful attention needs to be paid to the characteristics of the ground motion records used when analyzing the dynamic response behaviour of a structure. Once a suitable seed record or suite of seed records has been selected, however, the peak response probability distributions for a given structural model could be applied to a real structure with reasonable confidence since a coefficient of variation in the structural parameters of between 0.2 and 0.3 was required before any significant affect on the peak elastic response distributions was observed. Finally, results from the analysis of the accuracy of the RSA procedure showed, as expected, that the ABSSUM modal combination rule gives a very conservative estimate of peak response and that the SRSS and CQC error distribution is well represented by the Normal 
distribution. Using the properties of the Normal distribution, approximate rules-ofthumb were established for the accuracy of the CQC and SRSS modal combination rules for peak base shear, peak base moment and peak fifth storey displacement. The observed standard deviations in the distributions of the peak responses indicated that; $2 / 3$ of the time the CQC and SRSS results for peak base shear will be within 10\% of the RHA result, while peak base moment and peak fifth storey displacement will be within $5 \%$ of the RHA result.

The second case study analyzed the equal displacement observation in structural dynamics as well as the effect of hysteresis model properties on displacement response using a group of single-degree-of-freedom structures with eight different natural periods and $2 \%$ damping subjected to a sequence of 1000 generated records using six different hysteresis models. The hysteresis models for the structures were derived from experimental data taken from a cyclic lateral displacement-controlled test of a Parallam ${ }^{\circledR}$ column, which was analyzed using the hysteresis parameter identification feature of PSResponse. Dynamic analysis results showed that the Gumbel distribution is again a good description of the random behaviour of peak displacement response and, therefore, it was used as the basis for comparison in evaluating the effect of the different hysteresis models as well as the agreement between peak elastic and peak inelastic displacement.

To quantify the effect of different hysteresis models on peak inelastic displacement response, the reliability index $\beta$ of each hysteresis model associated with structural drift limits of; $0.5 \%, 1 \%, 2 \%$ and $4 \%$, was calculated for each of the eight natural periods. This type of analysis is an example of the intended purpose of PSResponse, which is to enable the evaluation of structural reliability under earthquake loading. The calculated reliability indices showed three key trends in the drift exceedence probabilities. Firstly, for each drift limit at each natural period the elastic response gives a conservative estimate of drift reliability as evidenced by the smaller $\beta$ value compared to each of the six hysteresis models. Secondly, the $\beta$ values for the hysteresis models vary significantly at a given natural period and drift limit. Thirdly, the 
range of variability in the $\beta$ values for the hysteresis models is dependent on the natural period and chosen drift limit.

The latter two key observations indicate that the characteristics of a hysteresis model have a significant effect on the calculated seismic reliability of a structure, with the effect being more or less pronounced depending on the capacity limit that is used to assess seismic reliability. Taken together, these observations show that the hysteretic behaviour of a structure needs to be accurately modeled, particularly in shorter natural period structures, to provide an accurate probabilistic description of response and hence a good estimate of seismic structural reliability. The natural period dependence of the relative importance of an accurate hysteresis model is a consequence of the decreasing importance of the restoring force $F(x)$ in the equation of motion as structural mass increases for a given structural stiffness. Therefore, the shape of the hysteresis loop for longer period structures is relatively less important when determining the probabilistic response of the structure:

Results from the analysis of the equal displacement observation showed that the distribution of elastic peak displacements differed from the inelastic distribution for each hysteresis model for all natural periods exhibiting a significant response. In general, the modes of the inelastic distributions were located at a lower peak displacement than the mode of the corresponding elastic distribution. This observed trend suggests that the elastic response of a structure tends to give a conservative estimate of peak inelastic displacement. To enable evaluation of this observed conservativeness and identify how it changes with natural period and relative yield strength, the distribution of percentage difference between the elastic and inelastic peak displacement was determined for each natural period using three hysteresis models; Hysteresis D, Hysteresis E and Hysteresis F, which represent non-degrading hysteretic behaviour with $100 \%$ yield strength, $50 \%$ yield strength and $25 \%$ yield strength, respectively.

Using Gumbel distributions to describe the distribution of percentage difference results, the probability that the percentage difference between peak inelastic and peak 
elastic displacement exceeded $0 \%$ was evaluated for each hysteresis model (i.e. yield level) at each natural period. This then allowed reliability indices to be calculated for the conservativeness of the equal displacement principle. Note that a negative percentage difference indicates that the peak elastic displacement exceeded the peak inelastic displacement, thereby giving a conservative estimate of peak displacement.

The calculated reliability indices for the conservativeness of the equal displacement principle showed that peak elastic displacement is generally a conservative estimate of peak inelastic displacement (elastic $\geq$ inelastic) except for longer natural period structures with reduced yield strength. Also, for a given natural period the probability that peak elastic displacement will give a conservative estimate of peak inelastic displacement (elastic $\geq$ inelastic) is reduced as yield strength decreases.

While it is difficult to make definitive statements regarding the accuracy of the equal displacement principle owing to the wide spread in percentage difference results for each hysteresis model at each natural period, the preceding observations indicate that the equal displacement principle is valid in the sense that elastic peak displacement provides a useful, generally conservative first approximation of inelastic peak displacement.

\subsection{CONCLUSIONS}

Developing a software application for evaluating the probabilistic response of a structural system to the stochastic dynamic action of an earthquake is a challenging process that depends on the successful integration of a number of mathematical modeling techniques that have been developed over the last several decades. Conclusions regarding the merits of the modeling methods and program functionality that were integrated into PSResponse as well as the contributions that have been made in the development of this research and analysis tool can be summarized with respect to the objectives that were set out at the beginning of this project. These objectives were: 
1. Evaluate the various analytical and numerical methods that have been developed to predict the response of linear and non-linear systems under stochastic dynamic actions.

2. Identify a method suitable for probabilistic analysis of the seismic response of civil engineering structures.

3. Develop software to enable application of the chosen probabilistic analysis method for use by engineers and researchers in evaluating structural reliability under seismic loading.

The first two objectives were met through a comprehensive review of the random vibration methods and numerical procedures that have been developed since the first application of probabilistic methods in the field of structural dynamics several decades ago. The theory and application of probabilistic methods is a vast field that crosses back and forth over traditional discipline boundaries between engineering, physics, and mathematics and every effort was made to identify and assess all available methods for their suitability in analyzing the probabilistic seismic response of civil engineering structures. That review now serves as a reasonably current state-of-the-art summary of probabilistic methods and the component models required in numerical procedures. From the review it was determined that the frequency-domain based random vibration methods are too restrictive in their inherent assumptions to confidently apply their results to real structures experiencing realistic earthquakes. Instead, a numerical time-history approach incorporating the Monte Carlo method provides a robust, accurate and straightforward means of evaluating the probabilistic response of a structure without regard to the degree of non-linearity in the restoring force, complexity of the structural system, nature of the variability in structural properties or nature of the random excitation process.

The third objective was met by developing a user-friendly, intuitive software tool that successfully integrates all the elements required in stochastic numerical modeling into what is believed to be the first software application of its kind. Engineers and researchers now have at their disposal a software application that can rigorously evaluate 
the probable effect of a wide range of ground motion characteristics and structural model parameters, each with their own random nature, on the dynamic response of a structure. This required that an entirely new, standalone application be developed to provide the computational speed necessary for Monte Carlo dynamic analysis of elastic and inelastic structures as well as the convenience of a Windows user-interface for easy access to the software.

To provide researchers the ability to evaluate the probabilistic response behaviour of experimental structural systems and connections, a new algorithm for parameter identification of the well-known BWBN, or Bouc-Wen, hysteresis model was developed for use with experimental data. This algorithm, and the non-linear analysis algorithms in general, also incorporated a modification to the BWBN model that was specifically made for this software application to simplify the parameter identification process. The number of parameters controlling pinching behaviour in the $\mathrm{BWBN}$ model was reduced from six to three, which has the added benefit that the role of each of the three new parameters is more easily understood than the relationship between the six parameters of the original pinching function.

For engineering design and analysis purposes, the probabilistic seismic response behaviour of a particular structure or structural system may be evaluated simply using the calculated lateral yield displacement of the structure and a specified post-yield relative stiffness. This allows the approximate probability distribution of seismic response behaviour to be determined without having experimental data available to fit the BWBN hysteresis model. The accuracy of this approximate distribution, which is based on an estimated yield strength and does not include the effect of degrading hysteretic behaviour, was investigated in a case study that is summarized shortly. Changes to a design affecting either the dynamic behaviour of the structure or the random distributions of the structural properties would currently require a new probabilistic analysis of the response behaviour, however, future development of PSResponse could incorporate a neural network or response surface application to reduce the requirement for new probabilistic analyses as design changes are made. 
Following the successful completion of the objectives of this project, a further contribution was made to the fields of structural dynamics and earthquake engineering through the completion of two case studies that also demonstrated the capabilities of PSResponse as a research and analysis tool. These case studies provided for the first time a probabilistic analysis of the importance of the hysteresis assumption in inelastic dynamic analysis, the accuracy of the equal displacement observation and the relative effect of random structural properties on elastic dynamic response. The general conclusions that were drawn within the context of the scope of the case studies were as follows:

1. The hysteretic behaviour of a structure needs to be accurately modeled, particularly in shorter natural period structures, to provide an accurate probabilistic description of response and hence a good estimate of seismic structural reliability.

2. The equal displacement principle is valid in the sense that elastic peak displacement provides a generally conservative first approximation of inelastic peak displacement, which in turn results in a generally conservative prediction of reliability.

3. The characteristics and randomness of ground motion records has a much larger influence than structural randomness on the probabilistic dynamic response of a structure. Therefore, once a suitable seed record has been selected, the peak response probability distributions for a given structural model could be applied to a real structure with reasonable confidence since the assumed level of uncertainty in the structural parameters needs to be only approximately correct. However, for strength related limit state evaluations related to peak response, structural variability still has an important effect. 


\subsection{RECOMMENDATIONS FOR FURTHER RESEARCH}

There are a virtually unlimited number of studies that could be carried out to evaluate the probable response of a wide range of structural models with various combinations of structural properties possessing different random distributions. These studies could include evaluations of; base isolation systems, optimal mass and damping distributions, first-passage probabilities and the temporal evolution of probabilistic response distributions. These types of analysis are possible with PSResponse but were not included in the case studies done as part of this project.

Future development of PSResponse could include the addition of several postprocessing features that would automate the statistical analysis of dynamic response results as well as link the results to a neural network or response surface application for structural optimization and design that conforms to specific performance measures. 


\section{REFERENCES}

Abrahamson, N.A., "Spatial Variation of Multiple Support Inputs", Proceedings of the $1^{\text {st }}$ U.S. Seminar on Seismic Evaluation and Retrofit of Steel Bridges, University of

California at Berkeley, San Francisco, October 1993.

Adams, J., Weichert, D., Halchuk, S., and Basham, P., "Trial Seismic Hazard Maps of Canada - 1995: Final Values for Selected Canadian Cities", Geological Survey of Canada, Ottawa, Ontario, Open File 3283.

Adomian, G., Stochastic Systems, Academic Press, London, 1983.

Akaike, H., “A New Look at Statistical Model Identification”, IEEE Transactions on Automation and Control, 19, 716-723, 1979.

Amin, M., and Ang, A.H.-S., "Nonstationary Stochastic Models of Earthquake", Journal of Engineering Mechanics, ASCE, 94(EM2), 559-583, 1968.

Aoki, S., "Stationary Approximation Method for Seismic Response Analysis to NonStationary Earthquake Motions", Proceedings of ICOSSAR '97 - The $7^{\text {th }}$ International Conference on Structural Safety and Reliability, Kyoto, November 24-28, 1997.

Atalik, T.S. and Utku, S., "Stochastic Linearization of Multidegree of Freedom Nonlinear Systems", Journal of Earthquake Engineering and Structural Dynamics, 4, 411-420, 1976.

Atkinson, G., "Use of the Uniform Hazard Spectrum in Characterizing Expected Levels of Seismic Ground Shaking", Proceedings, $6^{\text {th }}$ Canadian Conference on Earthquake Engineering, Toronto, 469-476, 1991.

Atkinson, G.A., and Beresnev, I.A., "Compatible Ground-Motion Time Histories for New National Seismic Hazard Maps", Canadian Journal of Civil Engineering, 25(2), 305-318, 1998.

Atkinson, J.D., "Eigenfunction Expansions for Randomly Excited Nonlinear Systems", Journal of Sound and Vibration, 30(2), 153-172, 1973.

Austin, M.A., Pister, K.S., and Mahin, S.A., "Probabilistic Design of Moment-Resistant Frames Under Seismic Loading", Journal of Structural Engineering, 113(8), 1660-1677, 1987.

Ayyub, B.M., and Chia, C.-Y., "Generalized Conditional Expectation for Structural Reliability Assessment", Structural Safety, 11(2), 131-146, 1991.

Ayyub, B.M., and Haldar, A., "Practical Structural Reliability Techniques", Journal of Structural Engineering, ASCE, 110(8), 1707-1724, 1984. 
Ayyub, B.M., and Kwan-Ling Lai, "Structural Reliability Assessment Using Latin Hypercube Sampling”, Proceedings of ICOSSAR ' 89 - The $5^{\text {th }}$ International Conference on Structural Safety and Reliability, San Francisco, August 7-11, 1989.

Baber, T.T., "Nonzero Mean Random Vibration of Hysteretic System", Journal of Engineering Mechanics, ASCE, 110(7), 1036-1049, 1984.

Baber, T.T., and Wen, Y.K., "Random Vibration of Hysteretic Degrading Systems", Journal of the Engineering Mechanics Division, ASCE, 107(EM6), Proc. Paper 16712, 1069-1087, 1981.

Baber, T.T., and Noori, M.N., "Random Vibration of Degrading, Pinching Systems", ASCE Journal of Engineering Mechanics, 111(8), 1010-1026, 1985.

Baber, T.T., and Noori, M.N., "Modeling General Hysteresis Behaviour and Random Vibration Application", Journal of Vibration, Acoustics, Stress and Reliability in Design, 108, 411-420, 1986.

Bagchi, A., "Seismic Performance of Buildings Designed to National Building Code of Canada", $8^{\text {th }}$ Canadian Conference on Earthquake Engineering, Vancouver, 1999.

Basham, P.W., "Recent Advances in Understanding of Earthquake Potential and Seismic Hazards in Canada", $7^{\text {th }}$ Canadian Conference on Earthquake Engineering, Montreal, 1995.

Basu, B., and Gupta, V.K., "Non-Stationary Seismic Response of MDOF Systems by Wavelet Transfom", Earthquake Engineering and Structural Dynamics, 26, 1243-1258, 1997.

Basu, B., and Gupta, V.K., "Seismic Response of SDOF Systems by Wavelet Modeling of Non-Stationary Processes", Journal of Engineering Mechanics, 124(10), 1142-1150, 1998.

Bathe, K.J., Finite Element Procedures, Prentice-Hall, New Jersey, 1996.

Bedrosian, E., and Rice, S.O., “The Output Properties of Volterra Systems (Nonlinear Systems with Memory) Driven by Harmonic and Gaussian Inputs, Proc. IEEE, 59(12), 1688-1720, 1971.

Benaroya, H., "Decomposition Method of Adomian - Its Application to Problems of Structural Dynamics", Tech. Report No. 2, Weidlinger Associates, New York, 1984.

Bergman, L.A. and Spencer, B.F. Jr., "Solution of the First Passage Problem for Simple Linear and Nonlinear Oscillators by the Finite Element Method", Department of Theoretical and Applied Mechanics, University of Illinois at Urbana-Champaign, $T \&$ AM Report No. 461, 1983.

Bergman, L.A., and Spencer, B.F. Jr., "Robust Numerical Solution of the Transient Fokker-Planck Equation for Nonlinear Dynamical Systems", IUTAM Symposium on Nonlinear Stochastic Mechanics, Turin, Italy, July 1-5, 1991. 
Bhandari, R.G., and Sherrer, R.E., "Random Vibrations in Discrete Nonlinear Dynamic Systems”, Journal of Mechanical Engineering Science, 10(2), 168-174, 1968.

Bogdanoff, J.L., Goldberg, J.E., and Bernard, M.C., "Response of a Simple Structure to a Random Earthquake-Type Disturbance", Bulletin of the Seismological Society of America, 51(2), 293-310, 1961.

Bohm, F., and Bruckner-Foit, A., "On Criteria for Accepting a Response Surface Model”, Probabilistic Engineering Mechanics, 7, 183-190, 1992.

Bolotin, V.V., "Statistical Theory of the Aseismic Design of Structures", Proceedings of the $2^{\text {nd }}$ World Conference on Earthquake Engineering, Tokyo and Kyoto, Japan, II, $1365-1374,1960$.

Bolotin, V.V., "Seismic Risk Assessment for Structures with the Monte Carlo Simulation", Probabilistic Engineering Mechanics, 8, 169-177, 1993.

Botoon, R.C. Jr., "Nonlinear Control Systems with Random Inputs", IRE Trans. Circuit Theory, CT-1, 9-18, 1954.

Bouc, R., "Forced Vibration of Mechanical System with Hysteresis", abstract, Proceedings of the $4^{\text {th }}$ Conference on Nonlinear Oscillation, Prague, Czechoslovakia, 1967.

Box, G.E.P., and Jenkins, G.M., Time Series Analysis: Forecasting and Control, Holden Day, San Francisco, 1970.

Box, G.E.P., and Wilson, K.B., "On the Experimental Attainment of Optimum Conditions", Journal of the Royal Statistical Society, B(13), 1-38, 1951.

Branstetter, L.J., Jeong, G.D., Yao, J.T.P., Wen, Y.K., and Lin, Y.K., "Mathematical Modelling of Structural Behaviour During Earthquakes", Probabilistic Engineering Mechanics, 3(3), 130-145, 1988.

Brenner, C.E., and Bucher, C., "A Contribution to the SFE-Based Reliability Assessment of Nonlinear Structures Under Dynamic Loading”, Probabilistic Engineering Mechanics, 10, 265-273, 1995.

Brenner, $\dot{C}$. E., and Bucher, C., "Reliability Analysis of Nonlinear Structures Using Stochastic Finite Elements", Probabilistic Mechanics \& Structural Reliability:

Proceedings of the $7^{\text {th }}$ Specialty Conference, Worcester Massachusetts, August 7-9, 1996.

Bucher, C.G., and Brenner, C.E., "Dynamic Response of Uncertain Two-Dimensional Structures", Probabilistic Mechanics and Structural and Geotechnical Reliability, Y.K. Lin, ed., 132-135, 1992.

Bulleit, W.M., and Decator, D.B., "Reliability Analysis of Bolted Wood Connections", Probabilistic Mechanics \& Structural Reliability: Proceedings of the $7^{\text {th }}$ Specialty Conference, Worcester Massachusetts, August 7-9, 1996. 
Burden, R.L., Faires, J.D., Numerical Analysis, $3^{\text {rd }}$ Ed., Prindel, Weber \& Schmidt, Boston, 1985.

Bycroft, G.N., "White Noise Representation of Earthquakes", Journal of the Engineering Mechanics Division, ASCE, 86(2), 1-16, 1960.

Cai, G.Q., "Random Vibration of Nonlinear Systems under Nonwhite Excitations", Journal of Engineering Mechanics, 121, 633-639, 1995.

Cai, G.Q., and Lin, Y.K., “A New Approximate Solution Technique for Randomly Excited Nonlinear Oscillators", International Journal of Nonlinear Mechanics, 23, 409420, 1988.

Cai, G.Q., and Lin, Y.K., "Reliability of Dynamical Systems Under Non-Gaussian Random Excitations", Proceedings of ICOSSAR '97 - The $7^{\text {th }}$ International Conference on Structural Safety and Reliability, Kyoto, November 24-28, 1997.

Cai, G.Q., and Lin, Y.K., “Response and Reliability of Nonlinear Systems Under NonGaussian Excitations", Proceedings of the $4^{\text {th }}$ International Conference on Stochastic Structural Dynamics, Notre Dame, Indiana, August 6-8, 1998.

Cai, G.Q., and Lin, Y.K., "Strongly Nonlinear System Under Non-White Random Excitations", Proceedings of the $4^{\text {th }}$ International Conference on Stochastic Structural Dynamics, Notre Dame, Indiana, August 6-8, 1998.

Casciati, F., "Nonlinear Stochastic Dynamics of Large Structural Systems by Equivalent Linearization", Proceedings, $5^{\text {th }}$ International Conference on Application of Statistics and Probability in Soil and Structural Engineering, II, 1165-1172, ICASP 5, Vancouver, Canada, May 1987.

Casciati, F., and Faravelli, L., "Methods of Non-Linear Stochastic Dynamics for the Assessment of Structural Fragility”, Nuclear Engineering and Design, 90, 341-356, 1985.

Caughey, T.K., "Random Excitation of a System With Bilinear Hysteresis", Journal of Applied Mechanics, 649-652, 1960.

Caughey, T.K., "Derivation and Application of the Fokker-Planck Equation to Discrete Linear Dynamic Systems Subjected to White Noise Excitation”, Journal of the Acoustical Society of America, 35(11), 1683-1692, 1963.

Caughey, T.K., "Equivalent Linearization Techniques", Journal of the Acoustical Society of America, 35(11), 1706-1711, 1963.

Ceccotti, A., and Karacabeyli, E., "Seismic Performance of Moment Resisting Timber Frames", Proceedings of the $5^{\text {th }}$ World Conference on Timber Engineering, Montreaux, August 17-20, 1998.

Ceccotti, A., and Vignoli, A., "A Hysteretic Behavioural Model for Semi-Rigid Joints", European Earthquake Engineering, 3, 3-9, 1989. 
Chang, C.C., Ger, J.F., and Cheng, F.Y., "Reliability-Based Optimum Design for UBC and Non-Deterministic Seismic Spectra", Journal of Structural Engineering, 120, 139$160,1994$.

Chang, M.K., Kwiatkowski, J.W., Nau, R.F., Oliver, R.M., and Pister, K.S., "ARMA Models for Earthquake Ground Motions", Earthquake Engineering and Structural Dynamics, 10, 651-662, 1982.

Chiu, H.M., and Hsu, C.S., "A Cell Mapping Method for Nonlinear Deterministic and Stochastic Systems - Part II: Examples of Application", Journal of Applied Mechanics, 53, 702-710, 1986.

Chopra, A.K., Dynamics of Structures: Theory and Applications to Earthquake Engineering, Prentice Hall, New Jersey, 1995.

Chou, C., "Modeling of Nonlinear Stiffness and Nonviscous Damping in Nailed Joints Between Wood and Plywood", Ph.D. thesis, Oregon State University, Corvallis, Oregon, 1987.

Chui, Y.H., and Ni, C., "Dynamic Response of Timber Frames with Semi-Rigid Moment Connections", Proceedings, $7^{\text {th }}$ Canadian Conference on Earthquake Engineering, Montreal, 1995.

Clough, R.W., and Johnston, S.B., "Effect of Stiffness Degradation on Earthquake Ductility Requirements", Proceedings, $2^{\text {nd }}$ Japan Earthquake Engineering Symposium, Tokyo, Japan, 227-232, 1966.

Collins, K.R., Wen, Y.K., and Foutch, D.A., "Dual-Level Seismic Design: A ReliabilityBased Methodology", Earthquake Engineering and Structural Dynamics, 25, 1433-1467, 1996.

Computers \& Structures Inc., SAP2000 Nonlinear 8.1.2, January 2003.

Conte, J.P., "Effects of Earthquake Frequency Nonstationarity on Inelastic Structural Response", Proceedings of the $10^{\text {th }}$ World Conference on Earthquake Engineering, A.A. Balkema, Rotterdam, The Netherlands.

Conte, J.P., and Peng, B.F., "Fully Nonstationary Analytical Earthquake Ground-Motion Model", Journal of Engineering Mechanics, 123(1), 15-24, 1997.

Conte, J.P., Pister, K.S., and Mahin, S.A., "Stochastic Seismic Response of Simple Structures", Mechanics Computing in 1990's and Beyond, 651-655, 1991.

Cooley, J.W., and Tukey, J.W., "An Algorithm for the Machine Calculation of Complex Fourier Series", Mathematics of Computation, 19, 1965.

Cornell, C.A., "Stochastic Process Models in Structural Engineering", Technical Report No. 34, Department of Civil Engineering, Stanford University, 1960. 
Crandall, S.H., "Perturbation Techniques for Random Vibration of Non-Linear Oscillators", Journal of Acoustical Society of America, 35(11), 1700-1705, 1963.

Crandall, S.H., Chandiramani, K.L., and Cook, R.G., "Some First-Passage Problems in Random Vibration”, ASME Journal of Applied Mechanics, 33, 532-538, 1966.

Crandall, S.H., "First-Crossing Probabilities of the Linear Oscillator", Journal of Sound and Vibration, 12(3), 285-299, 1970.

Crandall, S.H., "Non-Gaussian Closure for Random Vibration of Non-Linear Oscillators", Journal of Non-Linear Mechanics, 15, 303-313, 1980.

Daubechies, I., "Ten Lectures on Wavelets", Society for Industrial and Applied Mathematics, Philadelphia, 1992.

Deam, B.L., and King, A., "The Seismic Behaviour of Timber Structures - State of the Art", Proceedings, $2^{\text {nd }}$ Pacific Timber Engineering Conference, Institute of Professional Engineers, Wellington, New Zealand, 1, 57-60, 1994.

Deodatis, G., "Non-Stationary Stochastic Vector Processes: Seismic Ground Motion Applications", Probabilistic Engineering Mechanics, 11, 149-168, 1996.

Deodatis, G., and Shinozuka, M., "Simulation of Seismic Ground Motion Using Stochastic Waves", Journal of Engineering Mechanics, ASCE, 115, 2723-2737, 1989.

Deodatis, G., Shinozuka, M., and Papageorgiou, A., "Stochastic Wave Representation of Seismic Ground Motion II: Simulation", Journal of Engineering Mechanics, ASCE, 116(11), 2381-2399.

Der Kiureghian, A., "Seismic Risk Analysis of Structural Systems", Journal of Engineering Mechanics, ASCE, 107(6), 1133-1153, 1981.

Dey, A., and Mahadevan, S., "Ductile Structural System Reliability Analysis Using Adaptive Importance Sampling”, Structural Safety, 20, 137-154, 1998.

Dobson, S.D., Noori, M.N., and Hou, Z., "Boolean Modeling of Mechanical Hysteretic Systems", Proceedings of ICOSSAR '97 - The $7^{\text {th }}$ International Conference on Structural Safety and Reliability, Kyoto, November 24-28, 1997.

Dolan, J.D., "The Dynamic Response of Timber Shear Walls", Ph.D. thesis, University of British Columbia, Vancouver, BC, Canada, 1989.

Dowrick, D.J., "Hysteresis Loops for Timber Structures", Bulletin of the New Zealand National Society of Earthquake Engineering, Waikanoe, New Zealand, 19(20), 143-152, 1986.

EERI Committee on Seismic Risk, "The Basics of Seismic Risk Analysis", Earthquake Spectra, 5, 675-702, 1989.

Ellingwood, B.R., "Probability-Based LRFD for Engineered Wood Construction", Structural Safety, 19(1), 53-65, 1997. 
Ewing, R.D., Healey, T.J., and Agbabian, M.S., "Seismic Analysis of Wood Diaphragms in Masonry Buildings", Proceedings, Workshop on Design of Horizontal Wood Diaphragms: ATC-7-1, Applied Technology Council, Redwood City, California, 253276.

Fan, F.-G., and Ahmadi, G., "Nonstationary Kanai-Tajimi Models for El Centro 1940 and Mexico 1985 Earthquakes", Probabilistic Engineering Mechanics, 5(4), 171-181, 1990.

Faravelli, L., "Response-Surface Approach for Reliability Analysis", Journal of Engineering Mechanics, 115(12), 2763-2781, 1989.

Faravelli, L., "Stochastic Finite Elements and Reliability Analysis", Probabilistic Mechanics and Structural and Geotechnical Reliability, Y.K. Lin, ed., 45-48, 1992.

Florian, A., "An Efficient Sampling Scheme: Updated Latin Hypercube Sampling”, Probabilistic Engineering Mechanics, 7, 123-130, 1992.

Foliente, G.C., "Hysteresis Modeling of Wood Joints and Structural Systems", Journal of Structural Engineering, 121(6), 1013-1022, 1995.

Foliente, G.C., "Issues in Seismic Performance Testing and Evaluation of Timber Structural Systems", Proceedings of the International Wood Engineering Conference, New Orleans, October 28-31, 1996.

Foliente, G.C., Singh, M.P., and Dolan, J.D., "Response Analysis of Wood Structures Under Natural Hazard Dynamic Loads", Wood and Fiber Science, 28(1), 110-127, 1996.

Foliente, G.C., Singh, M.P., and Noori, M.N., "Equivalent Linearization of Generally Pinching Hysteretic, Degrading Systems", Earthquake Engineering and Structural Dynamics, 25, 611-629, 1996.

Foliente, G.C., Paevere, P., and Ma, F., "Parameter Identification and Seismic Response Analysis of Timber Structures", Proceedings of the $5^{\text {th }}$ World Conference on Timber Engineering, Montreaux, August 17-20, 1998.

Foschi, R.O., Folz, B.R., and Yao, F.Z., "Reliability-Based Design of Wood Structures", Structural Research Series, Report No. 34, Dept. of Civil Engineering, University of British Columbia, 1989.

Freeman, S.A., "Trying to Define Performance-Based Seismic Engineering - Is There a Consensus?", Proceedings, $8^{\text {th }}$ Canadian Conference on Earthquake Engineering, Vancouver, 1999.

Frenette, C., Foschi, R.O., and Prion, H.G.L., "Dynamic Behaviour of Timber Frame with Dowel Type Connections", Proceedings, International Wood Engineering Conference, New Orleans, 4, 89-96, 1996.

Ghanem, R.G., and Spanos, P.D., "Spectral Stochastic Finite--Element Formulation for Reliability Analysis", Journal of Engineering Mechanics, 117(10), 2351-2372, 1990. 
Ghanem, R., and Shinozuka, M., "Structural System Identification I: Theory", Journal of Engineering Mechanics, 121(2), 255-264, 1995.

Grigoriu, M., "Structural Response to Uncertain Seismic Excitations", Journal of Structural Engineering, 112(6), 1355-1365, 1986.

Grigoriu, M., "A Spectral Representation Based Model for Monte Carlo Simulation", Probabilistic Engineering Mechanics, 15, 365--370, 2000.

Grigoriu, M., Ruiz, S.E., and Rosenblueth, E., "The Mexico Earthquake of September 19, 1985 - Nonstationary Models of Seismic Ground Acceleration", Earthquake Spectra, 4(3), 551-568, 1988.

Haldar, A., and Zhou, Y., "Reliability of Nonlinear Frame Structures by SFEM", Probabilistic Mechanics and Structural and Geotechnical Reliability, Y.K. Lin, ed., 336$339,1992$.

Haldar, A., and Gao, L., "Reliability Evaluation Using SFEM", Proceedings of the $7^{\text {th }}$ Specialty Conference on Probabilistic Mechanics and Structural Reliability, Worcester, Massachusetts, August 7-9, 1996.

Han, S.W., and Wen, Y.K., "Method of Reliability-Based Seismic Design. I: Equivalent Nonlinear Systems", Journal of Structural Engineering, 123(3), 256-263, 1997.

Hao, H., Oliveira, C.S., and Penzien, J., "Multiple-Station Ground Motion Processing and Simulation Based on SMART-1 Array Data", Nuclear Engineering and Design, 111, 293-310, 1989.

Harris, C.J., "Simulation of Multivariable Non-Linear Stochastic Systems", International Journal for Numerical Methods in Engineering, 14, 37-50, 1979.

Hasofer, A.M., Ditlevsen, O., and Tarp-Johansen, N.J., "Positive Random Fields for Modeling Material Stiffnes̀s and Compliance", Proceedings of ICOSSAR '97 - The $7^{\text {th }}$ International Conference on Structural Safety and Reliability, Kyoto, November 24-28, 1997.

Heidebrecht, A.C., "Seismic Level of Protection for Building Structures", Canadian Journal of Civil Engineering, 24, 20-33, 1997.

Heidebrecht, A.C., Basham, P.W., and Finn, W.D.L., "Overview of Major Issues Involved in Preparing New Seismic Loading Provisions for the 2000 Edition of the National Building Code of Canada", Proceedings, $7^{\text {th }}$ Canadian Conference on Earthquake Engineering, Montreal, 1995.

Heidebrecht, A.C., Naumoski, N., and Rutenberg, A., "Towards a New Base Shear Format in Future NBCC Seismic Provisions", Canadian Journal of Civil Engineering, 21, 682-695, 1994.

Hendrickson, E.M., Ellingwood, B., and Murphy, J., "Limit State Probabilities for Wood Structural Members", ASCE Journal of Structural Engineering, 113(1), 88-106, 1987. 
Hockey, B., Prion, H.G.L., Lam, F., and Popovski, M., "Ductile Timber Connections for Earthquake Resistant Design", Proceedings, $8^{\text {th }}$ Canadian Conference on Earthquake Engineering, Vancouver, 1999.

Hong, H.P., and Lind, N.C., "Approximate Reliability Analysis Using Normal Polynomial and Simulation Results", Structural Safety, 18(4), 329-339, 1996.

Housner, G.W., "Characteristics of Strong-Motion Earthquakes", Bulletin of the Seismological Society of Amerïca, 37(1), 19-31, 1947.

Housner, G.W., and Jennings, P.C., "Generation of Artificial Earthquakes", Journal of Engineering Mechanics, ASCE, 90(EM1), 113-150, 1964.

Hsu, C.S., "A Theory of Cell-to-Cell Mapping Dynamical Systems", ASME Journal of Applied Mechanics, 47, 931-939, 1980.

Hsu, C.S., and Guttalu, R.S., "An Unravelling Algorithm for Global Analysis of Dynamical Systems: An Application of Cell-to-Cell Mappings", ASME Journal of Applied Mechanics, 47, 940-948, 1980.

Hsu, C.S., "A Generalized Theory of Cell-to-Cell Mapping for Nonlinear Dynamical Systems”, ASME Journal of Applied Mechanics, 48, 634-642, 1981.

Hsu, C.S., Guttalu, R.S., and Zhu, W.H., "A Method of Analyzing Generalized Cell Mappings", ASME Journal of Applied Mechanics, 49, 885-894, 1982.

Hsu, C.S., Chiu, H.M., “A Cell Mapping Method for Nonlinear Deterministic and Stochastic Systems - Part I: The Method of Analysis", ASME Journal of Applied Mechanics, 53, 695-701, 1986.

Humar, J.L., and Rahgozar, M.A., "Application of Inelastic Response Spectra Derived from Seismic Hazard Spectral Ordinates for Canada", Canadian Journal of Civil Engineering, 23, 1051-1063, 1996.

Huntington, D.E., and Lyrintzis, C.S., "Improvements to and Limitations of Latin Hypercube Sampling”, Probabilistic Engineering Mechanics, 13(4), 245-253, 1998.

Hwang, H.H.M., and Huo, J., "Generation of Hazard-Consistent Ground Motion", Soil Dynamics and Earthquake Engineering, 13(6), 377-386, 1994.

Ibidapo-Ope, O., and Diiwu, J.Y., " A Martingale Approach to the Reliability of a Structural System Under Nonstationary Excitation", Journal of Mathematical Analysis and Applications, 127, 403-412, 1987.

Imam, R.L., and Conover, W.J., "Small Sample Sensitivity Analysis Techniques for Computer Models with an Application to Risk Assessment", Communications in Statistics, A9(17), 1749-1842, 1980.

Itô, K., "On Stochastic Differential Equations", Memoirs of the American Mathematical Society, 4, 51-89, 1951. 
Iwan, W.D., "A Generalization of the Concept of Equivalent Linearization", International Journal of Non-Linear Mechanics, 8, 279-287, 1973.

Iwan, W.D., "Estimating Inelastic Response Spectra from Elastic Spectra", Earthquake Engineering and Structural Dynamics, 8, 375-388, 1980.

Iwan, W.D. and Lutes L.D., "Response of the Bilinear Hysteretic System to Stationary Random Excitation", Journal of the Acoustic Society of America, 43(3), 545-552, 1968.

Iwankiewicz, R., and Nielsen, S.R.K., "Dynamic Response of Hysteretic Systems to Poisson-Distributed Pulse Trains", Probabilistic Engineering Mechanics, 7, 135-148, 1992.

Iyengar, R.N., "Study of the Random Vibration of Nonlinear Systems by the Gaussian Closure Technique", ASME Journal of Applied Mechanics, 45, 393-399, 1978.

Iyengar, R.N., and Iyengar, K.T.S., "A Nonstationary Random Process Model for Earthquake Accelerograms", Bulletin of the Seismological Society of America, 59(3), 1163-1188, 1969.

Jaeger, L.G., Mufti, A.A., and Newhook, J.P., "A Note on Design Life and Safety Index for Seismic Design of Bridges and Structures", Canadian Journal of Civil Engineering, 24, 323-325, 1997.

Johnson, E.A., Bergman, L.A., Goldberg, D.E., and Dyke, S.J., "Monte Carlo Simulation of Dynamical Systems of Engineering Interest in a Massively Parallel Computing Environment: An Application of Genetic Algorithms", Proceedings of the IUTAM '95 Symposium on Advances in Nonlinear Stochastic Mechanics, Trondheim, Norway, July 3-7, Kluwer Academic, Dortrecht, The Netherlands, 225-234, 1996.

Johnson, E.A., Wojtkiewicz, S.F., Bergman, L.A., and Spencer, B.F. Jr., “Observations With Regard to Massively Parallel Computation for Monte Carlo Simulation of Stochastic Dynamical Systems", International Journal of Non-Linear Mechanics, 32(4), 721-734, 1997.

Kahn, H., "Use of Different Monte Carlo Sampling Schemes", Proceedings of the Symposium on Monte Carlo Methods, H.A. Meyer (Ed.), John Wiley \& Sons, New York, 149-190, 1956.

Kameda, H., and Nojima, N., "Simulation of Risk-Consistent Earthquake Motion", Earthquake Engineering and Structural Dynamics, 16, 1007-1019, 1988.

Kamiya, F., "Nonlinear Earthquake Response Analysis of Sheathed Wood Walls by a Computer Actuator On-line System", Proceedings, 1988 International Conference on Timber Engineering, Forest Products Society, Madison, Wisconsin, 1, 838-847.

Kanai, K., "Semi-Empirical Formula for the Seismic Characteristics of the Ground", Bulletin of the Earthquake Research Institute, University of Tokyo, 35, 308-325, 1957. 
Karacabeyli, E., and Ceccotti, A., "Seismic Design Considerations on Wood Frame Structures", Proceedings, $8^{\text {th }}$ Canadian Conference on Earthquake Engineering, Vancouver, 1999.

Kareem, A., Deodatis, G., and Shinozuka, M., "Modeling of Coherence for Stochastic Representation of Wind, Wave, and Seismic Load Effects", Proceedings of ICOSSAR '97 - The $7^{\text {th }}$ International Conference on Structural Safety and Reliability, Kyoto,

November 24-28, 1997.

Kennedy, D.J.L., and Medhekar, M.S., “A Proposed Strategy for Seismic Design of Steel Buildings”, Canadian Journal of Civil Engineering, 26, 564-571, 1999.

Khasminskii, R.Z., "Principle of Averaging for Parabolic and Elliptic Differential Equations and for Markov Processes with Small Diffusion", Theory of Probability \& Applications, 8, 1-21, 1963.

Khasminksii, R.Z., "On the Behaviour of a Conservative System with Small Friction and Small Random Noise”, Prikladnaya Matematika i Mechanica, 28(5), 1126-1130, 1964.

Kivell, B.T., Moss, P.J., and Carr, A.J., "Hysteretic Modeling of Moment Resisting Nailed Timber Joints", Bulletin of the New Zealand National Society of Earthquake Engineers, 14(4), 233-245.

Korn, G.A., and Korn, T:M., Mathematical Handbook for Scientists and Engineers, Dover, 2000.

Kozin, F., “Autoregressive Moving Average Models of Earthquake Records", Probabilistic Engineering Mechanics, 3(2), 58-63, 1988.

Kubo, T., and Penzien, J., "Simulation of Three-Dimensional Strong Ground Motions Along Principal Axes, San Fernando Earthquake", Earthquake Engineering and Structural Dynamics, 7, 279-294, 1979.

Kuwamura, H., and Galambos, T.V., "Earthquake Load for Structural Reliability", Journal of Structural Engineering, 115(6), 1446-1462, 1989.

Landa, P.S., and Stratonovitch, R.L., "Theory of Stochastic Transitions of Various Systems Between Different States", Vestnik MGU (Proc. Moscow University), III(1), $33-45,1962$.

Langley, R.S., “A Finite Element Method for the Statistics of Non-linear Random Vibration", Journal of Sound and Vibration, 101(1), 41-54, 1985.

Langtangen, H.P., "A General Numerical Solution Method for Fokker-Planck Equations With Applications to Structural Reliability", Probabilistic Engineering Mechanics, 6(1), 33-48, 1991.

Latendresse, V., Foschi, R.O., Prion, H.G.L., and Ventura, C.E., "Behaviour of Timber Dowel Type Connections under Seismic Loading", Proceedings, $7^{\text {th }}$ Canadian Conference on Earthquake Engineering, Montreal, 1995. 
Lee, C.S., "A Composite Beam Finite Element for Seismic Analysis of Wood-Framed Buildings”, Ph.D. thesis, Oregon State University, Corvallis, Oregon, 1987.

Lee, S., "Inelastic Earthquake Response Characteristics of Strong Column-Weak Girder Frame Structures”, Structural Dynamics - EURODYN'96, Balkerna, Rotterdam, 1996.

Li, C., and Der Kiureghian, A., "Optimal Discretization of Random Fields for SFEM", Probabilistic Mechanics and Structural and Geotechnical Reliability, Y.K. Lin, ed., 2932, 1992.

Li, K., CANNY 99, October 1996.

Li, Y., and Kareem, A., "Simulation of Multivariate Nonstationary Random Processes by FFT”, Journal of Engineering Mechanics, ASME, 117, 1037-1058, 1991.

Lin, Y.K., "Application of Nonstationary Shot Noise in the Study of System Response to a Class of Nonstationary Excitations", Trans. ASME, Journal of Applied Mechanics, 30, 555-558, 1963.

Lin, Y.K., "Nonstationary Response of Continuous Structures to Random Loading", Journal of the Acoustical Society of America, 35, 222-227, 1963.

Lin, Y.K., "Nonstationary Excitation and Response in Linear Systems Treated as a Sequence of Random Pulses", Journal of the Acoustical Society of America, 38, 453-460, 1965.

Lin, Y.K, Probabilistic Theory of Structural Dynamics, McGraw-Hill, New York, 1967.

Lin, Y.K., and Yong, Y., “Evolutionary Kanai-Tajimi Earthquake Models”, Journal of Engineering Mechanics, 113(8), 1119-1137, 1987.

Lin, Y.K., and Cai, G.Q., "Some Thoughts on Averaging Techniques in Stochastic Dynamics", Probabilistic Engineering Mechanics, 15, 7-14, 2000.

Liu, P., and Liu, K., "Selection of Random Field Mesh in Finite Element Reliability Analysis", Journal of Engineering Mechanics, 119(4), 667-680, 1993.

Liu, S., "Earthquake Response Statistics of Nonlinear Systems", Journal of the Engineering Mechanics Division, Proceedings of the ASCE, 95(EM2), 397-419, 1969.

Liu, S., "Synthesis of Stochastic Representations of Ground Motions", Bell System Technical Journal, 49, 521-541, 1970.

Ljung, L., System Identification: Theory For The User $2^{\text {nd }}$ Ed., Prentice Hall, New Jersey, 1999.

Lyon, R.H., "On the Vibration Statistics of a Randomly Excited Hard-spring Oscillator II", Journal of the Acoustical Society of America, 33(10), 1395-1403, 1961.

Mahadevan, S., and Haldar, A., "Stochastic FEM-Based Validation of LRFD", Journal of Structural Engineering, 117(5), 1393-1412, 1991. 
Mahadevan, S., and Mehta, S., "Stochastic Finite Element Based Reliability Analysis of Dynamically Loaded Frames", Mechanics Computing in 1990's and Beyond, 178-182, 1991.

Mahin, S.A., and Bertero, V.V., "An Evaluation of Inelastic Seismic Design Spectra", Journal of the Structural Division, Proceedings of the ASCE, 107(ST9), 1777-1795, 1981.

Marek, P., Gustar, M., Teply, B., Novak, D., and Kersner, Z., "Sensitivity Analysis in Simulation-Based Reliability Assessment of Structures", Proceedings of ICOSSAR '97 The $7^{\text {th }}$ International Conference on Structural Safety and Reliability, Kyoto, November 24-28, 1997.

Masri, S.F., and Caughey, T.K., "A Nonparametric Identification Technique for Nonlinear Dynamic Problems", ASME Journal of Applied Mechanics, 46, 433-447, 1979.

Masri, S.F., Sassi, H., and Caughey, T.K., "Nonparametric Identification of Nearly Arbitrary Nonlinear Systems", ASME Journal of Applied Mechanics, 49, 619-628, 1982.

Masri, S.F., Miller, R.K., Saud, A.F., and Caughey, T.K., "Mean-Square Response of Hysteretic Oscillators Under Nonstationary Random Excitation", Nonlinear Stochastic Dynamic Engineering Systems, IUTAM Symposium, Innsbruck, Austria, June 21-26, 1987.

Melchers, R.E., Structural Reliability Analysis and Prediction, John Wiley \& Sons, 1999

Melnik-Melnikov, P.G., and Dekhtyaruk, E.S., "Rare Events Probabilities Estimation by "Russian Roulette and Splitting" Simulation Technique", Probabilistic Engineering Mechanics, 15, 125-129, 2000.

Meskouris, K., and Kratzig, W.B., "Nonlinear Seismic Response of Reinforced Concrete Frames”, Nonlinear Stochastic Dynamic Engineering Systems, IUTAM Symposium, Innsbruck, Austria, June 21-26, 1987.

Micaletti, R.C., Cakmak, A.S., Nielsen, S.R.K., and Koyluglu, H.U., "A Solution Method for Linear and Geometrically Nonlinear MDOF Systems With Random Properties Subject to Random Excitation", Probabilistic Engineering Mechanics, 13(2), 85-95, 1998.

Minai, R., and Suzuki, Y., "Stochastic Estimates of Nonlinear Dynamic Systems", Proceedings, US-Japan Joint Seminar on Stochastic Approaches in Earthquake Engineering, Boca Raton, Florida, May 1987, 204-230.

Miyazawa, K., "Study on Nonlinear Static and Dynamic Structural Analysis of Wooden Wall Frame Buildings Subjected to Horizontal Force", Proceedings, $13^{\text {th }}$ Symposium on Computer Technology of Information, Systems and Applications, Architectural Institute of Japan (AIJ), 1990.

Myers, R.H., Response Surface Methodology, Allyn and Bacon Inc., Boston, 1976. 
Naeim, F., Lew, M., "On the Use of Design Spectrum Compatible Time Histories", Earthquake Spectra, 11(1), 111-127, 1995.

Nakayama, T., and Fujiwara, H., "A Simulation Method of Earthquake Ground Motion in Consideration of Time-Varying Phase Based on Nonstationary Spectrum Theory", Proceedings of ICOSSAR '97 - The $7^{\text {th }}$ International Conference on Structural Safety and Reliability, Kyoto, November 24-28, 1997.

Nau, J.M., and Hall,W.J., "Scaling Methods for Earthquake Response Spectra", Journal of Structural Engineering, 110(7), 1533-1548, 1984.

Naumoski, N., and Heidebrecht, A.C., "Implications of Preliminary Seismic Hazard Spectral Ordinates for Design Values in the National Building Code of Canada", Proceedings, $7^{\text {th }}$ Canadian Conference on Earthquake Engineering, Montreal, 1995.

Newland, D.E., "Wavelet Analysis of Vibration, Part 1: Theory", Journal of Vibration and Acoustics, 116, 409-416, 1994a.

Newland, D.E., "Wavelet Analysis of Vibration, Part 2: Wavelet Maps", Journal of Vibration and Acoustics, 116, 417-425, 1994 b.

Newmark, N.M., and Hall, W.J., "Procedures and Criteria for Earthquake Resistant Design”, Building Practice for Disaster Mitigation, Building Science Series 46, National Bureau of Standards, Washington, D.C., 209-236, 1973.

Nigam, N.C., and Narayanan, S., Applications of Random Vibrations, Springer-Verlag, 1994.

Papadimitriou, C., "Stochastic Characterization of Strong Ground Motion and Applications to Structural Response", Report No. EERL 90-03, California Institute of Technology, 1990.

Park, S.K., and Miller, K.W., Communications of the ACM, vol. 31, 1192-1201, 1988.

Park, Y.J., Wen, Y.K., and Ang, A.H-S., "Two-Dimensional Random Vibration of Hysteretic Structures, Journal of Earthquake Engineering and Structural Dynamics, 14, 543-557, 1986.

Polhemus, N.W., and Cakmak, A.S., "Simulation of Earthquake Ground Motions Using Autoregressive Moving Average (ARMA) Models", Earthquake Engineering and Structural Dynamics, 9, 343-354, 1981.

Popovski, M., Karacabeyli, E., and Prion, H.G.L., "Dynamic Response of Braced Timber Frames", Proceedings, $8^{\text {th }}$ Canadian Conference on Earthquake Engineering, Vancouver, 1999.

Pradlwarter, H.J., and Schueller, G.I., "On Advanced Monte Carlo Simulation Procedures in Stochastic Structural Dynamics", International Journal of Non-Linear Mechanics, 32(4), 735-744, 1997. 
Pradlwarter, H.J., and Schueller, G.I., "Assessment of Low Probability Events of Dynamical Systems by Controlled Monte Carlo Simulation", Probabilistic Engineering Mechanics, 14, 213-227, 1999.

Pradlwarter, H.J., Schueller, G.I., and Melnik-Melnikov, P.G., "Reliability of MDOFSystems", Probabilistic Engineering Mechanics, 9, 235-243, 1994.

Prakash, V., Powell, G.H., and Campbell, S., DRAIN-2DX Base Program Description and User Guide: Version 1.10, Report UCB/SEMM-93/17, Department of Civil Engineering, University of California, Berkeley, 1993.

Press, W.H., Teukolsky, S.A., Vetterling, W.T., Flannery, B.P., Numerical Recipes in C: The Art of Scientific Computing $2^{\text {nd }} E d$., Cambridge University Press, Cambridge, U.K., 1999.

Priestley, M.B., "Evolutionary Spectra and Non-Stationary Processes", Journal of the Royal Statistical Society, Series B, 27, 204-237, 1965.

Priestley, M.B., "Power Spectral Analysis of Non-Stationary Random Processes", Journal of Sound and Vibration, 6(1), 86-97, 1967.

Racicot, R.L., "Random Vibration Analysis - Application to Wind Loaded Structures", Solid Mechanics, Structures and Mechanical Design Division Report No. 30, Case Western Reserve University, 1969.

Ramadan, O., and Novak, M., "Simulation of Spatially Incoherent Random Ground Motions", Journal of Engineering Mechanics, ASCE, 119, 997-1016, 1993.

Ramberg, W., and Osgood, W.R., "Description of Stress-Strain Curves by Three Parameters", NACA Technical Note, TN-902, 1943.

Rice, S.O., "Mathematical Analysis of Random Noise, Bell System Technical Journal, 23, 282-332 (1944), 24, 46-156 (1945). Reprinted in "Selected Papers on Noise and Stochastic Processes”, N. Wax (ed.), 133-294, Dover, New York, 1954.

Roberts, J.B., "The Response of an Oscillator with Bilinear Hysteresis to Stationary Random Excitation", Journal of Applied Mechanics, 45, 923-928, 1978.

Roberts, J.B., "Response of Nonlinear Mechanical Systems to Random Excitation: Part 2: Equivalent Linearization and Other Methods", The Shock and Vibration Digest, 13(4), $17-28,1981$.

Roberts, J.B., "Energy Method for Nonlinear Systems with Non-White Excitation", IUTAM Symposium on Random Vibration and Reliability, Berlin, Akademie-Verlag, 285-294, 1982.

Roberts, J.B., and Spanos, P.D., "Stochastic Averaging: An Approximate Method of Solving Random Vibration Problems", International Journal of Non-Linear Mechanics, 21(2), 111-134, 1986. 
Roberts, J.B., Dunne, J.F., and Debonos, A., "A Spectral Method for Estimation of NonLinear System Parameters from Measured Response", Probabilistic Engineering Mechanics, 10, 199-207, 1995.

Rosowsky, D.V., "Computational Damage Modeling for Timber Reliability Analysis”, Mechanics Computing in 1990's and Beyond, 308-312, 1991.

Rosowsky, D.V., and Ellingwood, B., "Limit-State Interactions in Reliability-Based Design for Wood Structures", Journal of Structural Engineering, 118(3), 813-827, 1992.

Rubinstein, R., Simulation and the Monte Carlo Method, John Wiley \& Sons, New York, 1981.

Ruiz, P., and Penzien, J., "Stochastic Seismic Response of Structures", Journal of Engineering Mechanics, ASCE, 97(EM2), 441-456, 1971.

Ruxton, S., "Dynamic Performance of the Expanded Steel Tube Connection", M.A.Sc. thesis, University of British Columbia, Vancouver, B.C., 2003.

Rychlik, I., "On the 'Narrow-Band' Approximation for Expected Fatigue Damage", Probabilistic Engineering Mechanics, 8, 1-4, 1993.

Rychlik, I., Lindgen, G., and Lin, Y.K., "Markov Based Correlations of Damage Cycles in Gaussian and Non-Gaussian Loads", Probabilistic Engineering Mechanics, 10, 103$115,1995$.

Safak, E., "Optimal-Adaptive Filters for Modelling Spectral Shape, Site Amplifications and Source Scaling", Soil Dynamics and Earthquake Engineering, 8, 75-95, 1989.

Safak, E., and Boore, D.M., "On Nonstationary Stochastic Models for Earthquakes", Proceedings of the $3^{\text {rd }}$ U.S. National Conference on Earthquake Engineering, Charleston, South Carolina, August 24-28, 1986.

Sakamoto, I., and Ohashi, Y., "Seismic Response and Required Lateral Strength of Wooden Dwellings", Proceedings, 1988 International Conference on Timber Engineering, Forest Products Society, Madison, Wisconsin, 2, 243-247.

Shinozuka, M., "Monte Carlo Solution of Structural Dynamics", Journal of Computers and Structures, 2, 855-874, 1972.

Shinozuka, M., and Jan, C.M., "Digital Simulation of Random Processes and its Applications", Journal of Sound and Vibration, 25(1), 111-128, 1972.

Shinozuka, M., Deodatis, G., and Wu, W.F., "Nonlinear Dynamic Response and System Stochasticity", Nonlinear Stochastic Dynamic Engineering Systems, IUTAM Symposium, Innsbruck, Austria, June 21-26, 1987.

Shinozuka, M., and Deodatis, G., "Stochastic Process Models for Earthquake Ground Motion", Probabilistic Engineering Mechanics, 3(3), 114-123, 1988. 
Shinozuka, M., and Ghanem, R., "Structural System Identification II: Experimental Verification", Journal of Engineering Mechanics, 121(2), 265-273, 1995.

Shinozuka, M., and Sato, Y., "Simulation of Nonstationary Random Processes", Journal of Engineering Mechanics, ASCE, 93(EM1), 11-40, 1967.

Shinozuka, M., and Wen, Y.K., "Monte Carlo Simulation of Nonlinear Vibrations", American Institute of Aeronautics and Astronautics Journal, 10(1), 37-40, 1972.

Soize, C., "Steady-State Solution of Fokker-Planck Equation in Higher Dimension", Probabilistic Engineering Mechanics, 3(4), 196-206, 1988.

Sólnes, J., Stochastic Processes and Random Vibrations: Theory and Practice, John Wiley \& Sons, 1997.

Somerville, P., Smith, N., Punyamurthula, S., Sun, J., "Development of Ground Motion Time Histories for Phase 2 of the FEMA/SAC Steel Project", Report No. SAC/BD97/04, October 15, 1997.

Spanos, P.T.D., "Monte Carlo Simulations of Responses of Non-Symmetric Dynamic System to Random Excitations", Journal of Computers and Structures, 13, 371-376, 1981.

Spanos, P., and Zeldin, B., "Discretization Aspects of Stochastic Finite Elements", Proceedings of ICOSSAR '97 - The $7^{\text {th }}$ International Conference on Structural Safety and Reliability, Kyoto, November 24-28, 1997.

Spencer, B.F., Jr., "On the Reliability of Nonlinear Hysteretic Structures Subjected to Broad-Band Random Excitation", Lecture Notes in Engineering, C.A. Brebbia and S.A. Orszag ed., 21, Springer-Verlag, 1986.

Spencer, B.F. Jr., and Bergman, L.A., "On the Reliability of a Simple Hysteretic System", Journal of the Engineering Mechanics Division ASCE III, 1502-1514, 1985.

Spencer, B.F. Jr., and Bergman, L.A., "Numerical Solution of the Fokker-Planck Equation for First Passage Probabilities", Proceedings, First International Conference on Computational Stochastic Mechanics, Corfu, Greece, September 17-19, 1991.

Stephens, J.E., and Yao, J.T.P., "Damage Assessment Using Response Measurements", Journal of Structural Engineering, 113(4), 787-801, 1987.

Stewart, W.G., "The Seismic Design of Plywood Sheathed Shear Walls", Ph.D. thesis, University of Canterbury, Christchurch, New Zealand, 1987.

Stojadinovic, B., and Collins, K.R., "A Two-Dimensional Framework for PerformanceBased Seismic Design", Proceedings, $8^{\text {th }}$ Canadian Conference on Earthquake Engineering, Vancouver, 1999.

Stratonovitch, R.L., "Topics in the Theory of Random Noise", Gordon and Breach, New York, Vols. 1 and 2, 1964. 
Sucuoglu, H., Yucemen, S., Gezer, A., and Erberik, A., "Statistical Evaluation of the Damage Potential of Earthquake Ground Motions", Structural Safety, 20, 357-378, 1998.

Sues, R.H., Mau, S.T., and Wen, Y.K., "System Identification of Degrading Hysteretic Restoring Forces”, Journal of Engineering Mechanics, ASCE, 1145(5), 833-846.

Sues, R.H., Wen, Y.K., and Ang, A.H.S., "Stochastic Evaluation of Seismic Structural Performance", Journal of Structural Engineering, 111(6), 1204-1218, 1985.

Sun, J.Q., and Hsu, C.S., "First-Passage Time Probability of Non-linear Stochastic Systems by Generalized Cell Mapping Method", Journal of Sound and Vibration, 124(2), 233-248, 1988.

Sun, J.Q., and Hsu, C.S., "The Generalized Cell Mapping Method in Nonlinear Random Vibration Based Upon Short-Time Gaussian Approximation", ASME Journal of Applied Mechanics, 57, 1018-1025, 1990.

Suzuki, Y., and Araki; T., "Seismic Damage and Reliability Analysis of Uncertain Structural Systems", Proceedings of ICOSSAR '97 - The $7^{\text {th }}$ International Conference on Structural Safety and Reliability, Kyoto, November 24-28, 1997.

Suzuki, Y., and Minai, R., "Stochastic Seismic Damage and Reliability Analysis of Hysteretic Structures", Nonlinear Stochastic Dynamic Engineering Systems, IUTAM Symposium, Innsbruck, Austria, June 21-26, 1987.

Tajimi, H., "A Statistical Method of Determining the Maximum Response of a Building Structure During an Earthquake", Proceedings of the $2^{\text {nd }}$ World Conference on Earthquake Engineering, Tokyo and Kyoto, Japan, 781-797, 1960.

Takeda, T., Sozen, M.A., and Nielson, M.N., "Reinforced Concrete Response to Simulated Earthquakes", Journal of the Structural Division, ASCE, 96, 2557-2573, 1976.

Thomson, W.T., Theory of Vibration with Applications, Prentice Hall, New Jersey, 1988.

Timber Engineering Software, Department of Civil Engineering, University of British Columbia, Vancouver, BC, Canada, 1993.

Toland, R.H. and Yang, C.Y., "Random Walk Model for First Passage Probability", Journal of Engineering Mechanics Division, ASCE, 97(EM3), 791-807, 1971.

Trebicki, J., "Analysis of Stochastic Systems with Stiffness Degradation", Proceedings of the $4^{\text {th }}$ International Conference on Stochastic Structural Dynamics, Notre Dame, Indiana, August 6-8, 1998.

Tremblay, R., "Development of Design Spectra for Long-Duration Ground Motions From Cascadia Subduction Earthquakes", Canadian Journal of Civil Engineering, 25, 1078-1090, 1998. 
Uzumeri, S.M., Otani, S., and Collins, M.P., "An Overview of Canadian Code

Requirements for Earthquake Resistant Concrete Buildings", Canadian Journal of Civil Engineering, 5, 427-441, 1978.

Wen, Y.K., "Approximate Method for Nonlinear Random Vibration", Journal of the Engineering Mechanics Division, ASCE, 101(EM8), Proc. Paper 11500, 389-401, 1975.

Wen, Y.K., "Method for Random Vibration of Hysteretic Systems", Journal of the Engineering Mechanics Division, ASCE, 102(EM2), Proc. Paper 12073, 249-263, 1976.

Wen, Y.K., "Equivalent Linearization for Hysteretic Systems Under Random Excitation", Journal of Applied Mechanics, 47, 150-154, 1980.

Wen, Y.K., "Approximate Methods for Nonlinear Time-Variant Reliability Analysis", ASCE Journal of Engineering Mechanics, 113(12), 1826-1839, 1987.

Wen, Y.K., "Methods of Random Vibration for Inelastic Structures", Applied Mechanics Review, 42(2), 39-52, 1989.

Wen, Y.K., and Ang, A.H-S., "Inelastic Modeling and System Identification", Proceedings, Structural Safety Evaluation Based on System Identification Approaches, International Workshop, Lambrecht, Germany, July 1987, 142-160.

Wen, Y.K., and Chen, H.C., "System Reliability Under Time Varying Loads: I", Journal of Engineering Mechanics, 115(4), 808-839, 1989.

Wen, Y.K., and Chen, H.C., “On Fast Integration for Time Variant Structural Reliability”, Probabilistic Engineering Mechanics, ASCE, 2(3), 156-162, 1987.

Wen, Y.K., and Eliopoulos, D., "Method for Nonstationary Random Vibration of Inelastic Structures", Probabilistic Engineering Mechanics, 9, 115-123, 1994.

Wiener, N., Nonlinear Problems in Random Theory, Technology Press of MIT and John Wiley \& Sons, 1958.

Winterstein, S.R., "Nonlinear Vibration Models for Extremes and Fatigue", $A S C E$ Journal of Engineering Mechanics, 114(10), 1772-1790, 1988.

Wittig, L.E., and Sinha, A.K., "Simulation of Multi-Correlated Random Processes using FFT Algorithm", Journal of the Acoustic Society of America, 58(3), 630-634, 1975.

Wu, W.F., "Comparison of Gaussian Closure Technique and Equivalent Linearization Method”, Probabilistic Engineering Mechanics, 2(1), 2-8, 1987.

Wu, W.F., and Lin, Y.K., "Cumulant-Neglect Closure Scheme for Nonlinear Oscillators under Random Parametric and External Excitation", International Journal of Nonlinear Mechanics, 19(4), 349-362, 1984.

Wu, Y.T., "Structural Reliability Analysis Methods for Implicit Performance Functions", Probabilistic Mechanics and Structural and Geotechnical Reliability, Y.K. Lin, ed., 1992. 
Yamazaki, F., and Shinozuka, M., "Digital Generation of Non-Gaussian Stochastic Fields", Journal of Engineering Mechanics, ASCE, 114, 1183-1197, 1988.

Yang, J.N., "Simulation of Random Envelope Processes", Journal of Sound and Vibration, 25, 111-128, 1972.

Yang, J.N., and Liu, S.C., "Distribution of Maximum and Statistical Response Spectra", Journal of the Engineering Mechanics Division, Proceedings of the ASCE, 107(EM6), 1089-1101, 1981.

Yao, T.H.J., and Wen, Y.K., “Time-Variant System Reliability Analysis Using Response Surface Methodology and Fast Integration", Probabilistic Mechanics and Structural and Geotechnical Reliability, Y.K. Lin, ed., 1992.

Yasumura, M., "Seismic Behaviour of Arched Frames and Braced Frames", Proceedings, International Timber Engineering Conference, Tokyo, Japan, 3, 863-870, 1990.

Yasumura, M., "Evaluation of Seismic Performance of Timber Structures", Proceedings of the International Wood Engineering Conference, New Orleans, October 28-31, 1996.

Yeh, C.-H., and Wen, Y.K., "Modeling of Nonstationary Ground Motion and Analysis of Inelastic Response", Structural Safety, 8, 281-298, 1990.

Zahrah, T.F., and Hall, W.J., "Earthquake Energy Absorption in SDOF Structures", Journal of Structural Engineering, 110(8), 1757-1772, 1984.

Zerva, A., "Comparative Time Domain Analysis of Some Available Analytical and Empirical Expressions for the Spatial Variability of Seismic Ground Motion", Mechanics Computing in the 1990's and Beyond, 671-675, 1991.

Zhang, J., and Ellingwood, B., "SFEM for Reliability of Structures with Material Nonlinearities”, Journal of Structural Engineering, 122(6), 701-704, 1996.

Zhang, J., and Ellingwood, B., "SFEM in Nonlinear Structural Reliability Analysis", Proceedings of ICOSSAR '97 - The $7^{\text {th }}$ International Conference on Structural Safety and Reliability, Kyoto, November 24-28, 1997.

Zhang, R., and Shinozuka, M., "Statistical Seismic Responses of Structures Using Response Spectrum Matching Technique", Engineering Mechanics : Proceedings of the $11^{\text {th }}$ Conference, Fort Lauderdale, Florida, May 19-22, 1996.

Zhang, R., Yong, Y., and Lin, Y.K., "Earthquake Ground Motion Modeling II: Stochastic Line Source", Journal of Engineering Mechanics, ASCE, 117(9), 2133-2148.

Zhu, W.Q., "Stochastic Averaging Methods in Random Vibration", Applied Mechanics Review, 41(5), 189-199, 1988. 